\title{
NBSIR 74.453
}

(See NBSIR 73-235, Instruction Manual, Volume II)

Field Service Test Model: Computer-Controlled U System. Operations Manual and Software Operating System Appendices for the Automated System. Volume I.

\author{
K. M. Gray \\ J. R. Ellis
}

Institute for Computer Sciences and Technology National Bureau of Standards

Washington, D. C. 20234

January 1973

Instruction Manual

\section{Prepared for}

Department of the Air Force

HO - USAF (AFTAC/TAP)

Patrick A.F.B., Florida 32925 

NBSIR 74-453

(See NBSIR 73-235, Instruction Manual, Volume II)

FIELD SERVICE TEST MODEL:

COMPUTER-CONTROLLED U SYSTEM.

OPERATIONS MANUAL AND SOFTWARE

OPERATING SYSTEM APPENDICES FOR

THE AUTOMATED SYSTEM. VOLUME I.

K. M. Gray

J. R. Ellis

Institute for Computer Sciences and Technology

National Bureau of Standards

Washington, D. C. 20234

January 1973

Instruction Manual

Prepared for

Department of the Air Force

HQ - USAF (AFTAC/TAP)

Patrick A.F.B., Florida 32925

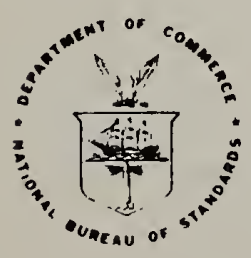

U. S. DEPARTMENT OF COMMERCE, Frederick B. Dent, Secretary 


\section{CONTENTS}

1. INTRODUCTION

- 1.1 General

1.2 Requirements

1.3 Hardware Description

1.4 Basic Data Flow

1.5 Basic Software Organization

1.6 Operating System

2. E\$INT - System Initialization Program

2.1 Functional Description

2.1.1 E:ANF - System Start/Restart Point

2.2 Called Subroutine

2.3 Input Parameter Descriptions

2.4 Output Parameter Descriptions

2.5 Internal Data Descriptions

2.5 Program Flow Chart

3. E\$SKD - System Scheduler

3.1 Functional Description

3.1.1 E:SKD - System Scheduler

3.1.2 E:BSK - Background Task Scheduler

3.2 Called Subroutines

3.3 Data Formats

3.4 Input Parameter Descriptions

3.5 Output Parameter Descriptions

3.6 Internal Data Descriptions

3.7 Program Flow Chart

4. E\$TTSI - Time Slicing Interrupt Program

4.1 Functional Description

4.1.1 E:TSI - Time Slicing Interrupt Service Routine

4.1.2 B:IOR - Background IO Request Routine

4.2 Called Subroutines

4.3 Input Parameter Descriptions

4.4 Output Parameter Descriptions

4.5 Program Flow Chart

5. E\$CLK - System Clock

5.1 Functional Descriptions

5.1.1 E:SEC - One Second Timing Interrupt Service Routine

5.1.2 E:MIN - One Minute Timing Interrupt Service Routine

5.2 Called Subroutines

5.3 Data Formats

5.4 Input Parameter Descriptions

5.5 Output Parameter Descriptions

5.6 Internal Data Descriptions

5.7 Program Flow Chart 

6. E\$UIN - U Data Input Program

6.1 Functional Description

6.1.1 E:ANL - AIS SI Interrupt Routine

6.1.2 E:UIN - U Data Preprocessing Routine

6.1.3 E:MIT - IOIS Digital Output Routine

6.2 Data Formats

6.2.1 AIS Input Data

6.2.2 Sensor Parameter Control Output via IOIS

6.2.3 DOY/TOD Input from WTC via IOIS

6.2.4 Amplitude Values

6.2.5 Phase Values

6.2.6 Blanking Rate Values

6.3 Input Parameter Descriptions

6.4 Output Parameter Descriptions

6.5 Internal Data Descriptions

6.6 Program Flow Chart

7. E\$̧SUB - System Monitor Subroutines

7.1 Functional Description

7.1.1 E:PIW - Power Loss Interrupt Service Routine

7.1.2 E:PWU - Power Restored Interrupt Service Routine

7.1.3 E:PAR - Memory Parity Error Interrupt Service Routine

7.1.4 E:ABR - Console Interrupt Service Routine

7.1.5 B:ORT - Background Task Abortion Routine

7.2 Called Subroutines

7.3 Input Parameter Descriptions

7.4 Output Parameter Descriptions

7.5 Internal Data Descriptions

7.6 Program Flow Chart 

FIELD SERVICE TEST MODEL: COMPUTER-CONTROLLED U SYSTEM

OPERATIONS MANUAL AND SOFTWARE OPERATING

SYSTEM APPENDICES FOR THE AUTOMATED SYSTEM

VOLUME I

\section{INTRODUCTION}

Volume I of the "Automated U System" describes the software 1istings and the operator's manual. This volume is intended to instruct the station technician in the procedure to load programs and operate, under computer control, the automated U system. Other manuals describe the system and the technical design and are contained in the following manuals:

Volume II. Field Service Test Model: Computer-Controlled U System. Operations Manual and Software Operating System Appendices for the Automated System.

Field Service Test Model: Computer-Controlled U System. Manual for Receiver and ADACS Interface Chassis.

Field Service Test Model: Computer-Controlled U System. Manual for Test Unit.

Field Service Test Model: Computer-Controlled U System. Manual for Computer Subsystem.

Field Service Test Model: Computer-Controlled U System. Manual for System.

Certain commercial equipment and materials are identified in this paper in order to adequately specify the components used. In no case does such identification imply recommendation or endorsement by the National Bureau of Standards, nor does it imply that the material or equipment identified is necessarily the best available for the purpose. 



\subsection{General.}

The New-U Service Test Model is the termination of a development project aimed at automating the $\mathrm{U}$ sensor functions. IBM completed initial algorithm definition on the IBM Model 360/75 computer for batch mode operations in April 1968. These programs covered the three sensors of the proposed $\mathrm{K}$ technique; $\mathrm{U}, \mathrm{Z2}$, and $\mathrm{H}$. In February 1969, a Scientific Control Corporation Model 660 computer was procured to permit the processing of data from the three sensors in real time. When the necessity to process data from all three sensors simultaneously was removed during the following year, a new Baseline Configuration was defined specifying simultaneous processing of the $\mathrm{U}$ and $\mathrm{Z} 2$ sensor data, but separate processing for the $\mathrm{H}$ sensor. In February 1970, the programs to implement this Baseline Configuration were completed and the system demonstrated. Post-baseline efforts concentrated on the $\mathrm{U}$ sensor alone. By September 1970, processing algorithms had been refined and experiments conducted to demonstrate the ability of the system to operate for extended periods of time without operator intervention. All efforts to that time had concentrated on the use of the current sensor 
hardware. By December 1970, the National Bureau of Standards and Computer Science Corporation (under contract to the USAF) had developed an experimental sensor front end with response characteristics far survassing those of the current equipment. The cost of using such new equipment was prohibitive, so effort was redirected toward modifying the existing equipment to permit faster response。 By June 1971, this experiment was completed and alternative Service Test Model configurations were defined. Due to the higher cost involved with the greater processing load necessary for the faster response configuration, the decision was made to use the standard outputs of the current sensor. The test model would handle fourteen channels of U sensor data simultaneously with one processor. Procurement specifications for the digital computer to be used in the Operational Service Test were completed in May 1972。 The contract was ultimately awarded to Modular Computer Systems Corporation for their MODCOMP III/5 digital computer with all necessary interface equipment and peripheral devices. The Processor was delivered to the Fort Belvoir experimental station in Virginia in mic-September, followed by the interface to the first two sensor channeis within a few weeks. The software package, including both the Real-Time Operating System (ROS) and the applications programs (both foreground and background), was named NUSTAD (New U System of Totally Automated 
Design). The skeleton of the ROS was on-line by mid-October。By the final week in November, the ROS was completed and the preliminary check-out of the applications programs was near completion。 The system was then shipped to McClellan AFB, CA where the remaining twelve sensor channels were interfaced and the first part of the Operational Service Test would be performed. Program checkout by the programming staff was completed during the last week of January 1973 , and the system was turned over to the 1155 th TOS to begin formal system evaluation/prior to sending the system to a remote overseas location for a nine month evaluation of system performance under actual field conditions.

This document fully describes the programming associated with NUSTAD。 The information contained herein should be sufficient to permit full understanding of the software operations and functions.

\subsection{Requirements.}

The primary goal of automating the $U$ sensor functions was to reduce manpower. To meet this goal, NUSTAD was required to perform the following operations:

a. Read sensor data in real-time at a rate consistent with processing algorithm requirements. 
b. Provide feedback control of the various parameters associated with the sensor as recessary to maintain sensor operations at an optimal level.

c. Perform waveform analysis of sensor data to recognize all signals of interest.

d. Monitor the status of sensor and interface equipment to recognize changes which affect processing operations and respond accordingly.

e. Maintain a station log of all status changes, parameter changes, and signals of interest, as well as periodic reports as required to evaluate sensor channel reliability。 Also, generate report messages suitable for transmission over standard communications lines。

f. Provide display and format checking functions to validate or review data from the DRFUD (Digital Recorder For U Data) data tapes. This will permit the use of existing equipment for bulk data storage independent of processor operations.

In addition to reducing manpower, the New U system will permit standardization of reporting methods, more effective utilization of existing equipment, and an extended useful life for current sensor equipment. 


\subsection{Hardware Description.}

The MODCOMP III/5 is the basic model of the MODCOMP II line. The III/ 5 used for the Service Test Model has the following characteristics:

\section{as Central Processing Unit}

1. Fifteen general purpose registers, all available for use as accumulators, seven available as index registers。

2. 800 nanosecond cycle time。

3. 167 instructions including bit, byte, and word manipulation。(See Appendix V for complete instruction lists)。

4. Hardware multiply/divide instructions.

5. Multi-level priority interrupt scheme。

b. Memory

1. 32, 768 words (16 bits) directly addressable, in four modules,

2. Memory parity (even parity) for each byte of memory。 c. Analog Input Subsystem

1. 48 channels multiplexed through a dedicated Direct Memory Processor (DMP) channel。

2. Input range of 0 to +10 volts.

3. Input in 11 bits plus sign, 
d. Digital Input/Output Interface Subsystem

1. 15 basic ( 16 bit) digital input channels, plus 8 (16 bit) digital input channels on the channel extender.

2. 16 (16 bit) output channels。

3. 8 user defined external interrupts.

e. Peripheral devices

1. ASR 35 Teletype (ASCII)。

2. ASR 28 Teietype (BAUDOT), configured for receive only operation。

3. Chalco 600 character/second paper tape reader.

4. Wang 45 ips magnetic tape unit。

The sensor is the standard EECO $881 \mathrm{M}$ Phase tracking receiver with modifications as described in an as yet unpublished NBS manual。 The ADACS (Automatic Data Analysis and Control System) interface unit is described in another as yet unpublished manual. The following list includes documents useful in understanding the software development:

$310-103000-000$

$310-600101$

$220-600500-000$

$210-600301-000$
MODCOMP II Reference Manual

MODC OMP Assembler Reference Mianual

MODCOMP Program Descriptions- Utility Programs

MODCOMP MAX I Reference Manual 


\section{0-341000 MODC OMP Tech Manual-Input/Output Interface Subsystem 1100 \\ $220-311000-001$ \\ MODCOMP Tech Manual-Analog Input Subsystem $1200 / 1500$}

1.4 Basic Data Flow

The essential elements of NUSTAD can be defined with respect to the systems hardware components through Figure 1。1. These elements are shown as they are related by the information flow among them。

Each U channel has the following input and output data for the processor:

as Three analog input channels. One each for Amplitude, Phase, and Blanking Percentage.

b. One digital input channel to supply the processor with the channel's frequency and cardioid settings, and the channel's ADACS INE, Maintenance, and AUTO/MANUAL conditions.

c。 One digital output channel supplying the receiver with the current RF gain, tracking rate, and blanking level data, and the phase shift control bito

The World Time Clock (WTC) supplies the following timing information to the processor: 


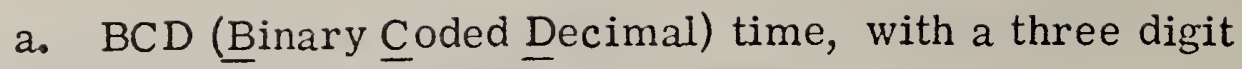
Day of Year (DOY) and a six digit Time of Day (TOD)。

b. One per second and one per minute time pulses to drive the interrupts used by the system clock routine。

c. Ten per second timing pulses to drive the time slicing program used to segment time for background tasks。

The ASR 35 is used as the primary operator/system communications device. It is the means by which the operator requests the various program options and supplies information necessary for the processing of the DRFUD data tapes。

The ASR 28 is used to maintain the station $\log _{\text {。 }}$ All messages or reports of any historical value are printed here. A five-level paper tape is also punched, permitting the automatic preparation of data messages for later transmission over standard communications channels。

DRFUD tapes are recorded concurrent to on-line processing by a modified DRFUD unit for each eight U sensor channels。 The tapes are played back into the system from the Wang tape drive, using background tasks requested by the operator. 
Figure 1.1 displays the relationship among the various IO signals and the individual programs using them. This diagram shows the relationship among programs on the basis of data flow, while Figure 1.2 shows how the programs are related by transfer of program control。

1.5 Basic Software Organization。

Linkages among the various programs are more easily understood by recognizing the naming conventions used for symbolic labels.

a. First character - The first character of a symbolic label tells to which basic function that particular label is associated:

1. E - used to identify operating system and executive support functions。

2. B - used to identify functions associated with background tasks other than DRFUD routines.

3. M - used to identify functions related to system status monitoring。

4. U and W - used to denote functions directly related to the $U$ data analysis functions.

5. F - used to identify functions asscciated with background DRFUD operations.

b. Second character - The second character of the label 


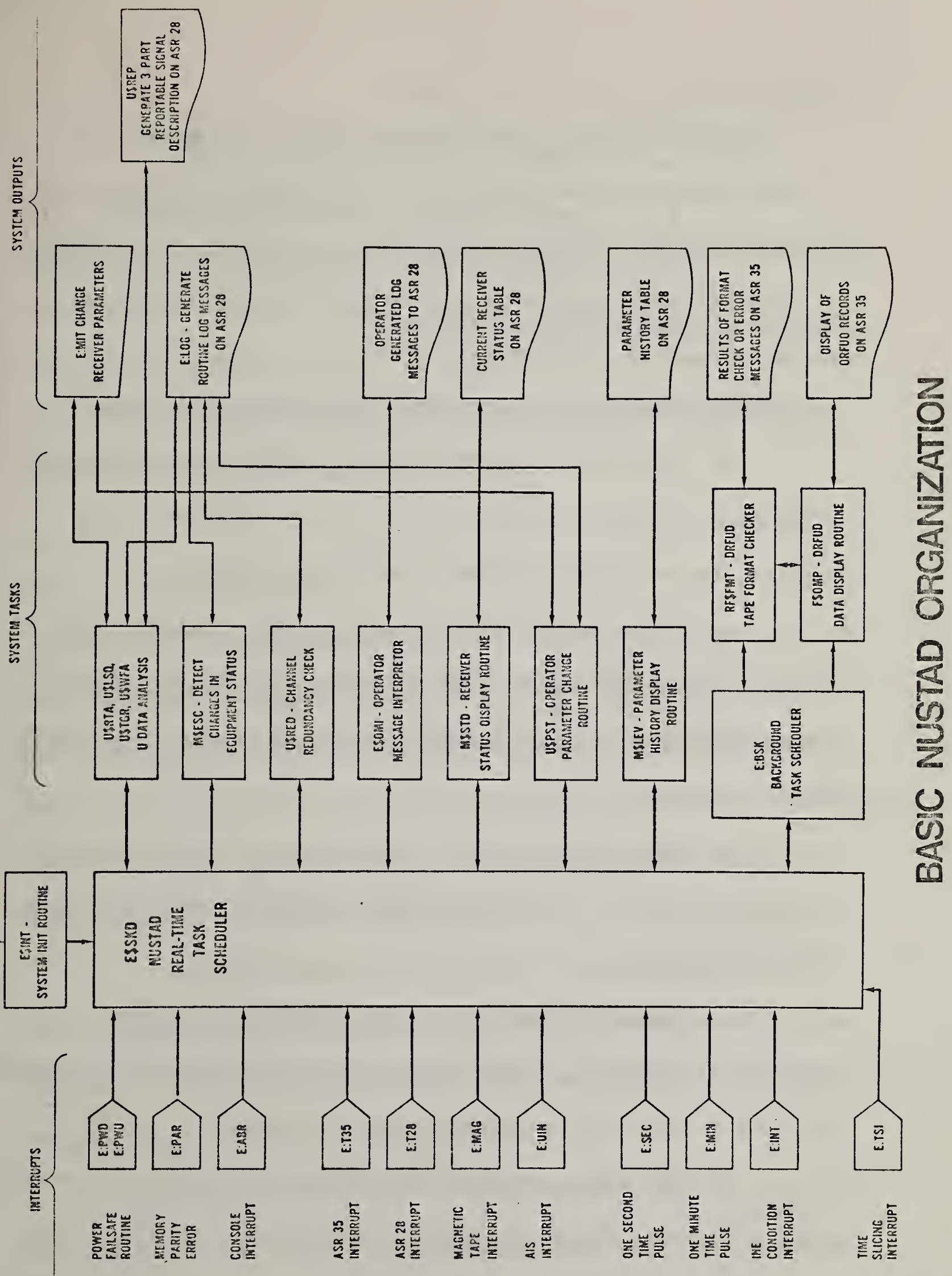


indicates how the labei is to be used:

1. $\$$ - used to denote a program name。

2. : - used to indicate a program entry point。

3. 。- used to specify a parameter location used in

another program (an external linkage)。

NUSTAD programs may be considered of five basic types:

a. Real-Time Operating System (ROS) - those programs

which control system resources; $e_{\circ} g_{\circ}$, the interrupt servicing routines, the peripheral handlers, and the schedulers.

b. Executive Support Programs - which perform utility

functions genorally needed by the applications programs, such as reformatting time or phase values into output format, computing the square root, etc。

c. Monitor Programs - which sense and report changes in hardware status and provide historic records needed to evaluate channel reliability。

d. U Analysis Programs - the primary applications programs of NUSTAD。 These programs provide automatic parameter control and real-time waveform analysis of $U$ data.

e. DRFUD Programs - These applications programs execute in the background level to provide some analysis of off-line $U$ data recorded by the DRFUD。 
The interaction among the programs of NUSTAD is shown schematically in Figure 1.2. The various tasks associated with the system's mission are scheduled in response to external interrupts. As these tasks execute, they use executive support functions to generate the system's output。 The scheduler is the heart of the ROS and NUSTAD as all program control is made through this program, including entry into the background state.

1.6 Operating System。

All NUSTAD programs are coded in the assembly language of the MODCOMP III computer, and are assembled under the manufacturer's MAX I batch oriented operating system。The programs are loaded using the manufacturer's Stand Alone Linking Loader (SAL, MODC OMP No。600000-012)。After loading, control is assumed by NUSTAD's ROS. NUSTAD runs in two basic levels:

a. FOREGROUND FUNC TIONS: These are the primary mission associated tasks which are scheduled in response to system interrupts。 These tasks are configured to execute to completion before returning control to the operating system.

b. BACKGROUND FUNC TIONS: These tasks are requested by the operator and execute subordinate to the foreground functions. 
Background tasks are interrupted frequently to give the for eground functions priority access to the system's resources。 These functions relinquish control to the operating system during all IO actions。 Only one background task is permitted to execute at a time. 
2。 E\$INT。

2.1 Functional Description

E\$INT is the NUSTAD system initialization routine. At load time (see Appendix III for loading procedures), it specifies the contents of low-core locations necessary for interrupt and IO operations. Table 2. 1 lists these locations and the values stored in them. Those specified as "DFC" contain the address of the symbolic label given。

\subsubsection{E:ANF (System Start/Restart Point)。}

E:ANF (location 010016) is the program execution start-up point。 Transferring control to NUSTAD from SAL by means of the GO command will cause execution to begin here. Also, depressing the MASTER CLEAR switch on the console panel will cause a system restart at this point。

When execution begins, E\$INT performs the following tasks:

a. Reset the start-up flag for the $U$ data acquisition program (E\$UIN)。

b. Transmit the current GAIN, TRACKING RATE, and BLANKING LEVEL values to the sensor.

c. Clear and activate all interrupts to be used。

d. Reset to initial values all processing flags, counters, parameters, etc。, used by the applications programs. 
e. Clear the channels to the ASR 35 and the ASR 28 .

f. Schedule M\$STD in scheduler。

go Send the system restart messages to the teleprinters;

a LF/CR to the ASR 35 and a "SYSTEM RESTART" message with the restart time to the ASR 28.

h. Establish initial values for background processing。

i. Transfer control to the system scheduler (E:SKD in (E\$SID)。

2. 2 Called Subroutines.

Subroutine Program Function

E:MIT E\$UIN Transmit GAIN, TRACKING RATE, and BLANKING LEVEL values to sensor channel given in $R 3$.

E:FRT E\$FRT Converts BCD DOY/TOD to ASCI characters for output.

E:035 E\$T35 Transfers output message to buffer before transmission to the ASR 35 .

E:028 E\$T28 Translates output message to BAUDOT code and stores in buffer before transmission to the ASR 28 
2.3 Input Parameter Descriptions.

Parameter Program Description

E。SF1 E\$UNN Bit $\varnothing$ set for first AIS record, reset thereafter。

U.CCN U\$DAT Accumulated effect of cycle shifts。

U.CPC U\$DAT Channel Compressed Point Counter。

U.CST U\$DAT Channel Status, set to 4 indicating channel down。

U.RTY U\$DAT Channel Recovery Tally, counts tensecond-periods before channel can come up。

U. IHB U\$DAT $\quad$ Bit $\varnothing$ set to inhibit processing on channel if INE or Maintenance set on the channel。

U. TM1 E\$CLK Minute counter for scheduling U\$RED。

U. TM3 U\$RTA Gain Clock, counts ten-second-periods for entry to gain control section of U\$RTA。

U. TM4 U\$RTA Track Clock, counts ten-second-periods for entry to tracking rate control section of U\$RTA。
E。035
$\mathrm{E} \$ \mathrm{~T} 35$
ASR 35 status word。
E。028
$\mathrm{E} \$ \mathrm{~T} 28$
ASR 28 status word。
E. Tø8 E\$SKD
Scheduler task table entry for M\$STD.
E。IOF $\quad \mathrm{E} \$ \mathrm{~T} 35$
Parameter word 1 sed in calling E:035。
E。28F
$\mathrm{E} \$ \mathrm{~T} 28$
Parameter word used in calling E:028。 
Parameter Program Description

U.AST U\$DAT Channel waveform analysis status, indicates point in signal to which processing has progressed, initially $\emptyset$ 。

F. MTP FSFMT Character pointer for Mag Tape data in input buffer。

B.STT E\$SKD Background Status Flag, set to $\emptyset$ for no task in progress.

E.TSF E\$SKD Time Slicing Flag, Bit $\varnothing$ set to $\varnothing$ to inhibit time-slice processing, set to 1 to indicate background task in execution and time-slicing needed.

E:SKD E\$SKD Entry to system scheduler。

2.4 Output Parameter Descriptions.

Parameter Size Description

E:ANF Entry point for system start/restarto

F:ANL Dummy background task; to be replaced by a background program to perform waveform analysis on data from DRFUD tapes。

2.5 Internal Data Descriptions.

Parameter Size Description

MSG $\varnothing \quad 39 \quad$ System restart message:

"......SYSTEM RESTART AT 123

123456...."

STIME

Location of DOY/TOD group in MSGØ。

MSG1

LF/CR from MSGø。 
Parameter Size

MSG8
Description

Message generated by $\mathrm{F}: \mathrm{ANL}$-"F:ANL CALLED" 


\begin{tabular}{|c|c|c|c|c|}
\hline Loc & \multicolumn{2}{|c|}{ Content } & Function & Program \\
\hline $\begin{array}{l}\varnothing \varnothing \varnothing \varnothing \\
\varnothing \varnothing \varnothing 1\end{array}$ & BRU & $\mathrm{E}: \mathrm{ANF}$ & Program restart point & E\$INT \\
\hline$\varnothing \emptyset 21$ & $\mathrm{DFC}$ & E:PWD & Power Down/Up Inter rupt Location & E\$SUB \\
\hline$\varnothing \not \varnothing 23$ & $\mathrm{DFC}$ & E:PAR & Memory Parity Error Interrupt Location & E\$SUB \\
\hline$\emptyset \not 3 \mathrm{D}$ & $\mathrm{DFC}$ & $E: A B R$ & CONSOLE Inter rupt Lo:ation & E\$SUB \\
\hline$\emptyset \not \partial 3 F$ & $\mathrm{DFC}$ & E:TSI & Time Slicing Interrupt Location & E\$TSI \\
\hline$\varnothing \varnothing 63$ & & & Transfer Count for AIS DMP & E\$UTN \\
\hline$\not \not \supset 64$ & & $\varnothing 8 \varnothing$ & Transfer Count for Mag Tape DMP & $\mathrm{E} \$ \mathrm{MAG}$ \\
\hline$\varnothing \not 73$ & $\mathrm{DFC}$ & $\mathrm{E} 。 \mathrm{UBU}$ & Transfer Address for AIS DMP & E\$UIN \\
\hline$\emptyset \emptyset 74$ & & $6_{16}$ & Transfer Address for Mag Tape DMP & $\mathrm{E} \$ \mathrm{MAG}$ \\
\hline$\varnothing \not 88 \mathrm{~A}$ & $\mathrm{DFC}$ & $\mathrm{E}: \mathrm{I} 35$ & Data Interrupt Location for ASR 35 & $\mathrm{E} \$ \mathrm{~T} 35$ \\
\hline$\varnothing \emptyset 98$ & $\mathrm{DFC}$ & $\mathrm{E}: \mathrm{I} 28$ & Data interrupt Location for ASR 28 & $\mathrm{E} \$ \mathrm{~T} 28$ \\
\hline$\varnothing \not \supset 99$ & $\mathrm{DFC}$ & $\mathrm{E}: I 28$ & Data Interrupt Location for ASR 28 & $\mathrm{E} \$ \mathrm{~T} 28$ \\
\hline$\varnothing \varnothing \mathrm{A} \varnothing$ & $\mathrm{DFC}$ & E:SEC & 1 per Second Interrupt Location & E\$CLK \\
\hline$\emptyset \emptyset \mathrm{A} 1$ & $\mathrm{DFC}$ & E:MIN & 1 per Minute Interrupt Location & $\mathrm{E} \$ \mathrm{CLK}$ \\
\hline$\varnothing \emptyset \mathrm{A} 2$ & $\mathrm{DFC}$ & M:INT & INE Interrupt Location (from ADACS) & M\$ESC \\
\hline$\emptyset \not \partial \mathrm{A} 7$ & $\mathrm{DFC}$ & M:INT & Common Alarm Board Interrupt Location & $\mathrm{M} \$ \mathrm{ESC}$ \\
\hline$\emptyset \not C 4$ & $\mathrm{DFC}$ & E:MTI & Service Interrupt Location for Mag Tape & E\$MAG \\
\hline$\varnothing \not \supset \mathrm{CA}$ & $\mathrm{DFC}$ & $\mathrm{E}: 35 \mathrm{~S}$ & Service Interrupt Location for ASR 35 & $\mathrm{E} \$ \mathrm{~T} 35$ \\
\hline$\not \not \varnothing \mathrm{D} 1$ & $\mathrm{DFC}$ & $\mathrm{E}: \mathrm{ANL}$ & Service Interrupt Location for AIS & E\$UTN \\
\hline$\emptyset 1 \varnothing \emptyset$ & & & Program Execution Starting Point & E\$INT \\
\hline
\end{tabular}

TABLE 2,1

Dedicated Memor y Locations Established in E EINT 
ANFNG

SET START FLAG E.SFI FOR USE IN ESUIN TO START DATA READ

\section{XMIT I}

TRANSMIT CURREVT DIGITAL RECEIVER

PARAMETERS TO

EQUIPMENT VIA

E:MIT (in ESUIN)

START UPINTERRUPTS ON LEVELS 0,1, $C, D, E, F$ \& EXTERNALS ON IOIS

\section{PINIT}

FOR EACH

CHANNEL, SET:

U. $C C N=\varnothing$

U. $C P C=\not \supset$

U.CST $=4$

U.RTY $=18$

U. $1 \mathrm{HB}=6^{\circ}$

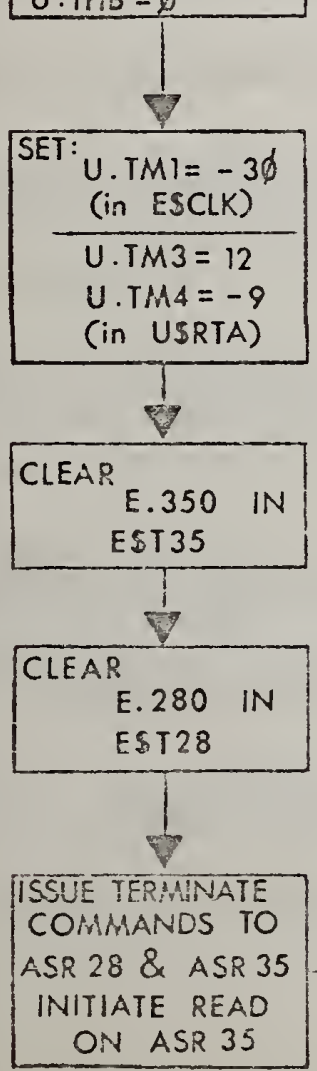

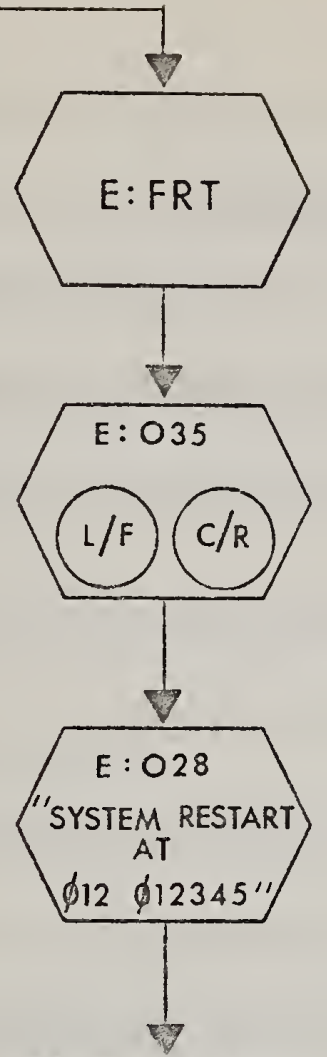

$F: A N L$

RESET MAG TAPE BUFFER POINTER TO INDUCE READING A NEW $=14080$

F. MTP $=1408$

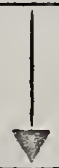

CLEAR BACKGROUND TASK.

SET B.STT $=\varnothing$

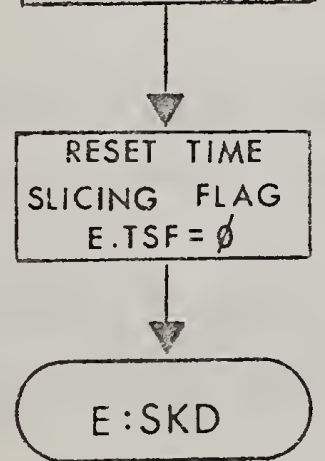

\section{$E: S K D$}


3. $\mathrm{E} \$ \mathrm{SKD}$ 。

3.1 Functional Description。

ESSKD is the heart of ROS。 The tasks in its task table are scheduled in response to the various external interrupts。 Through the task table, entry is then made to the various tasks in the system.

E\$SKD consists of two subprograms; E:SKD and E:BSK。 E:SKD performs three primary functions:

a. Control direct entry to the real-time tasks listed in the task table (priorities 1 through 14)。

b. Monitor CPU activity (primarily through interrupt actions) and $\log \operatorname{loss}$ of ROS control。

c. Control entry into the background level via priority 15 in the task table.

Control is passed to the system scheduler at E:SKD in one of four ways:

a, After a system restart, E\$INT unconditionally transfers control to E:SKD。

b. After each dedicated real-time task listed in the task tab!e completes execution, it returns control to the scheduler at $\mathrm{E}:$ SKD. 
c. When a time slicing interrupt occurs during the execution of a background task, E\$TSI retains the information needed to continue that task and returns control to E:SKD。

d. When a background task requests an IO action, B:IOR (in E\$TSI) retains the information needed to continue the task, and returns control to E:SKD until the IO action is completed,

\subsubsection{E:SKD (System Scheduler)。}

At E:SKD, the scheduler begins a scan of the task table to look for tasks which require servicing。 If one is found, the task flag bit is reset and control is passed to that task via the address stored as that task's entry in the task table. The tasks in the task table, and the program in which they may be found, are listed in Table 3.1 . Should no task be flagged during the pass through the task table, a counter is decremented, Sinould the counter reach $\emptyset$, indicating that approximately five seconds had elapsed without a scheduled task, the system is considered dead, and E\$LOG is called to generate a "T SD" message for the station log。A system restart is then attempted by passing control to $\mathrm{E}: \mathrm{ANF}$ in $\mathrm{E} \$ \mathrm{INT}$ 。 Each time a task is entered, then completed, control is returned at E:SirD and the counter is reset.

\subsubsection{E:BSK (Background Task Scheduler).}

When the flagged task is priority 15 , control is passed to $\mathrm{E}: \mathrm{BSK}$, 
the background task scheduler。 The status of the background task is stored in a word called B.STT (the Background Status)。 When the operator requests a background task, via E\$OMI (the Operator Message Interpretor), B. STT is set to a value corresponding to that task and E:BSK is flagged in the tas. table. Thus, the first time E:BSK is entered for a given task, it will find B.STT equal to 1, 2, or 3, and will transfer control to the desired task, as listed in Table 3.2 。 Thereafter, entry to E:BSK will be to continue a task previously interrupted, In this case, either E:TSI or B:IOR will have set B。STT to 4 to indicate that a background task is in progress, and will have retained the lozation counter and the register contents so as to continue processing at that point where the interrupt occurred。 Therefore, when E:BSK finds $\mathrm{B}_{\mathrm{S}} \mathrm{STT}=4$, it restores the register s to the values retained in holding buffers at B. RS1 (for registers 1 through 7) and $B_{0}$ RS2 (for registers $1 \varnothing$ through 15), and returris control to the next instruction of the background task at the location saved in $B$, RET。 Should the operator wish to abort his background task, the console interrupt routine ( $\mathrm{B}: \mathrm{ORT}$ in $\mathrm{E} \$ \mathrm{SUB}$ ) resets $\mathrm{B} . \mathrm{STT}$ to $\not \circ$. When $\mathrm{E}: \mathrm{BSK}$ finds $\mathrm{B} . \mathrm{S} T \mathrm{~T}=\emptyset$, it simply returns control to E:SKD without executing any background task or res:heduling the background level. Therefore, B.STT will normally be $\not \supset$ when no background task is bəing prozessed, 


\subsection{Called Subroutines,}

Subroutine Program Function

E:LOG E\$LOG Generate coded log message for ASR 28.

(Also see Tables 3.1 and 3.2 for tasks called by E:SKD and $\mathrm{E}: \mathrm{BSK})$

3.3 Data Formats。

The primary data entity in the scheduler is the task table entry, which is of the form:

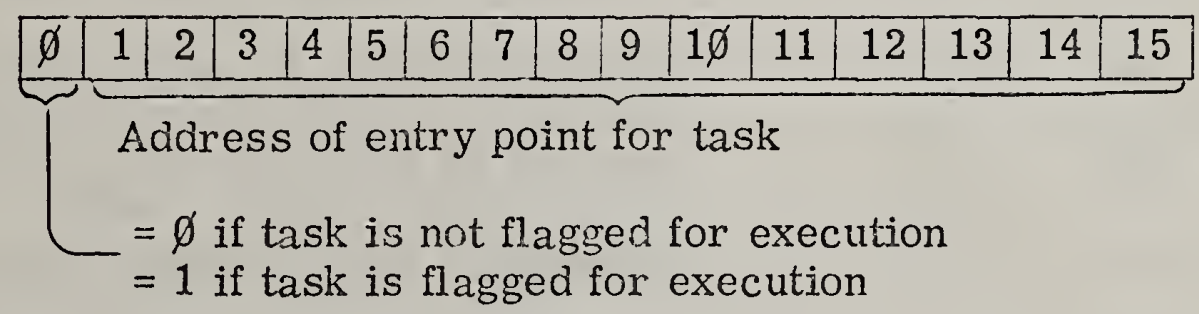

3.4 Input Parameter Descriptions.

Parameter Program Description

E:ANF E EINT $\quad$ Entry point for a system restart

3.5 Output Parameter Descriptions.

Parameter Size Description

E. TSK $1 \quad$ Base of task table

$\mathrm{E}:$ SKD

Entry to ROS scheduler

$\mathrm{E}: \mathrm{BSK}$

Entry to background level scheduler

3.4 


\begin{tabular}{|c|c|c|}
\hline Parameter & $\underline{\text { Size }}$ & Description \\
\hline $\mathrm{E}_{0} \mathrm{~T} \not 1$ & 1 & Priority 1 task tabie entry (see Table 3.1 ) \\
\hline $\mathrm{E}_{\circ}{ }^{\prime} \mathrm{\varnothing} \varnothing 2$ & 1 & Priority 2 task table entry (see Table 3.1 ) \\
\hline $\mathrm{E} . \mathrm{T} \emptyset \Omega$ & 1 & Priority 3 task table entry (see Table 3.1 ) \\
\hline $\mathrm{E} . \mathrm{T} \emptyset 4$ & 1 & Priority 4 task table entry (see Table 3.1 ) \\
\hline $\mathrm{E} 。 \mathrm{~T} \emptyset 5$ & 1 & Priority 5 task table entry (see Table 3.1 ) \\
\hline E.T Tø6 & 1 & Priority 6 task table entry (see Table 3.1 ) \\
\hline $\mathrm{E} . \mathrm{T} \varnothing 7$ & 1 & Priority 7 task table entry (see Table 3.1 ) \\
\hline $\mathrm{E}_{0} T \varnothing 8$ & 1 & Priority 8 task table entry (see Table 3.1 ) \\
\hline $\mathrm{E} . \mathrm{T} \emptyset 9$ & 1 & Priority 9 task table entry (see Table 3.1 ) \\
\hline $\mathrm{E} . \mathrm{T} 1 \varnothing$ & 1 & Priority $1 \varnothing$ task table entry (see Table 3.1 ) \\
\hline $\mathrm{E} 。 \mathrm{~T} 11$ & 1 & Priority 11 task table entry (see Table 3.1 ) \\
\hline $\mathrm{E} 。 \mathrm{~T} 12$ & 1 & Priority 12 task table entry (see Table 3.1 ) \\
\hline $\mathrm{E} . \mathrm{T} 13$ & 1 & Priority 13 task table entry (see Table 3.1 ) \\
\hline E。TI4 & 1 & Priority 14 task table entry (see Table 3.1 ) \\
\hline $\mathrm{E} . \mathrm{T} 15$ & 1 & Priority 15 task table entry (see Table 3.1 ) \\
\hline B. STT & 1 & Background Status (see Table 3.2 ) \\
\hline $\mathrm{E} 。 \mathrm{TSF}$ & 1 & $\begin{array}{l}\text { Time Slicing Flag: (see Section } 4.1 \text { ) } \\
=\varnothing \text { to inhibit processing of time slice } \\
\text { interrupt } \\
=1 \text { to permit processing of time slice } \\
\text { interrupt }\end{array}$ \\
\hline B. RS1 & 7 & $\begin{array}{l}\text { Holding buffer for registers } 1-7 \text { of } \\
\text { interrupted background task }\end{array}$ \\
\hline
\end{tabular}


Parameter Size Description

B. RS2 $6 \quad$ Holding buffer for registers $1 \varnothing-15$ of interrupted background task

B. RET 1 Return location for interrupted background task

3.6 Internal Data Descriptions,

Parameter Size Description

BTABL $5 \quad$ Table of background task entry points 


\begin{tabular}{|c|c|c|c|}
\hline Priority & $\begin{array}{c}\text { Program } \\
\text { Entry }\end{array}$ & $\begin{array}{l}\text { Program } \\
\text { Name }\end{array}$ & Task \\
\hline 1 & $\mathrm{U}: \mathrm{RTA}$ & U\$RTA & $\begin{array}{l}\text { U Data Real Time \& Waveform } \\
\text { Analysis }\end{array}$ \\
\hline 2 & $\mathrm{M}: \mathrm{ESC}$ & MSESC & $\begin{array}{l}\text { Scan for Equipment Status } \\
\text { Change }\end{array}$ \\
\hline 3 & E:UIN & E\$UIN & Preprocess last U Data Input \\
\hline 4 & U:RED & U\$RED & $\begin{array}{l}\text { Perform Channel Redundancy } \\
\text { Check }\end{array}$ \\
\hline 5 & $\mathrm{E}: \mathrm{T} 28$ & $\mathrm{E} \$ \mathrm{~T} 28$ & $\begin{array}{l}\text { ASR } 28 \text { Character Transfer } \\
\text { Ready }\end{array}$ \\
\hline 6 & $\mathrm{E}: \mathrm{OMI}$ & E\$OMI & Interpret Operator Message \\
\hline 7 & U:RP3 & U\$REP & Generate Part III RSD \\
\hline 8 & $M: S T D$ & M\$STD & $\begin{array}{l}\text { Generate Current Receiver } \\
\text { Status Table }\end{array}$ \\
\hline 9 & ----- & ----- & Unused \\
\hline $1 \varnothing$ & $\mathrm{B}: \mathrm{ORT}$ & E\$S.UB & Abort Background Task \\
\hline 11 & $\mathrm{E}: \mathrm{MAG}$ & $E \$ M A G$ & Mag Tape Transfer Complete \\
\hline 12 & $\mathrm{E}: \mathrm{T} 35$ & $\mathrm{E} \$ \mathrm{~T} 35$ & $\begin{array}{l}\text { ASR } 35 \text { Character Transfer } \\
\text { Ready }\end{array}$ \\
\hline 13 & $\mathrm{U}: \mathrm{PST}$ & U\$PST & $\begin{array}{l}\text { Process Operator Requested } \\
\text { Parameter Change }\end{array}$ \\
\hline 14 & M:LEV & MSTEV & $\begin{array}{l}\text { Generate Parameter History } \\
\text { Table }\end{array}$ \\
\hline 15 & $\mathrm{E}: \mathrm{BSK}$ & E\$SKD & $\begin{array}{l}\text { Enter Background Task Thru } \\
\text { E:BSK }\end{array}$ \\
\hline
\end{tabular}




\begin{tabular}{|c|c|c|c|}
\hline $\begin{array}{r}\text { B.STT } \\
\text { Code }\end{array}$ & $\begin{array}{l}\text { Program } \\
\text { Entry }\end{array}$ & $\begin{array}{l}\text { Program } \\
\text { Name }\end{array}$ & Task \\
\hline$\varnothing$ & & & Abort Background Processing \\
\hline 1 & F:FMT & F\$FMT & $\begin{array}{l}\text { DRFUD Tape Format Check } \\
\text { Program }\end{array}$ \\
\hline 2 & $\mathrm{~F}: \mathrm{DMP}$ & $\mathrm{F} \$ \mathrm{DMP}$ & DRFUD Data Display \\
\hline 3 & $\mathrm{~F}: \mathrm{ANL}$ & $\mathrm{E} \$ \mathrm{INT}$ & (Dummy Task) \\
\hline 4 & & & Continuation of Task in Progress \\
\hline
\end{tabular}

TABLE 3.2 NUSTAD BACKGROUND TASKS 


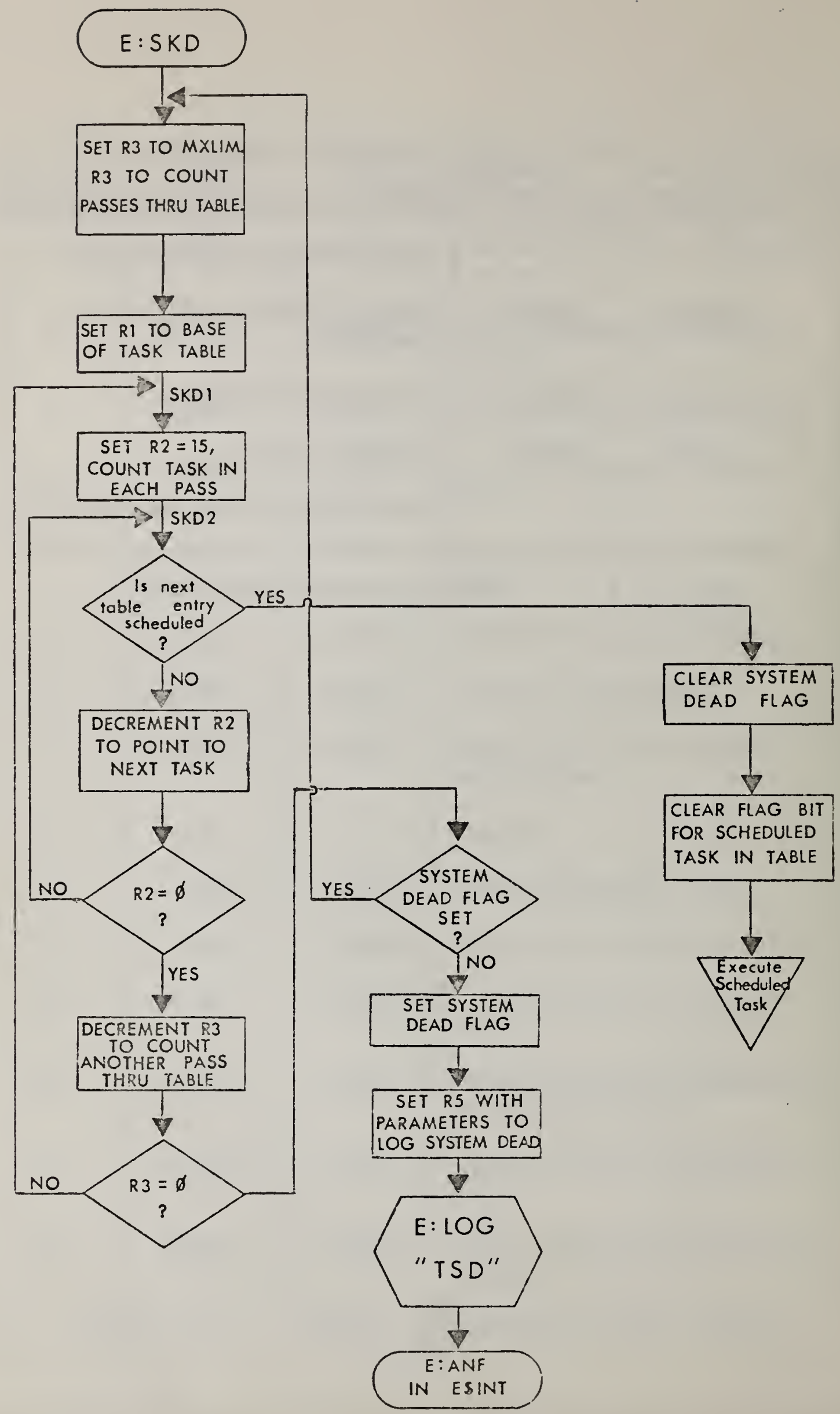

3. $8 \mathrm{a}$ 


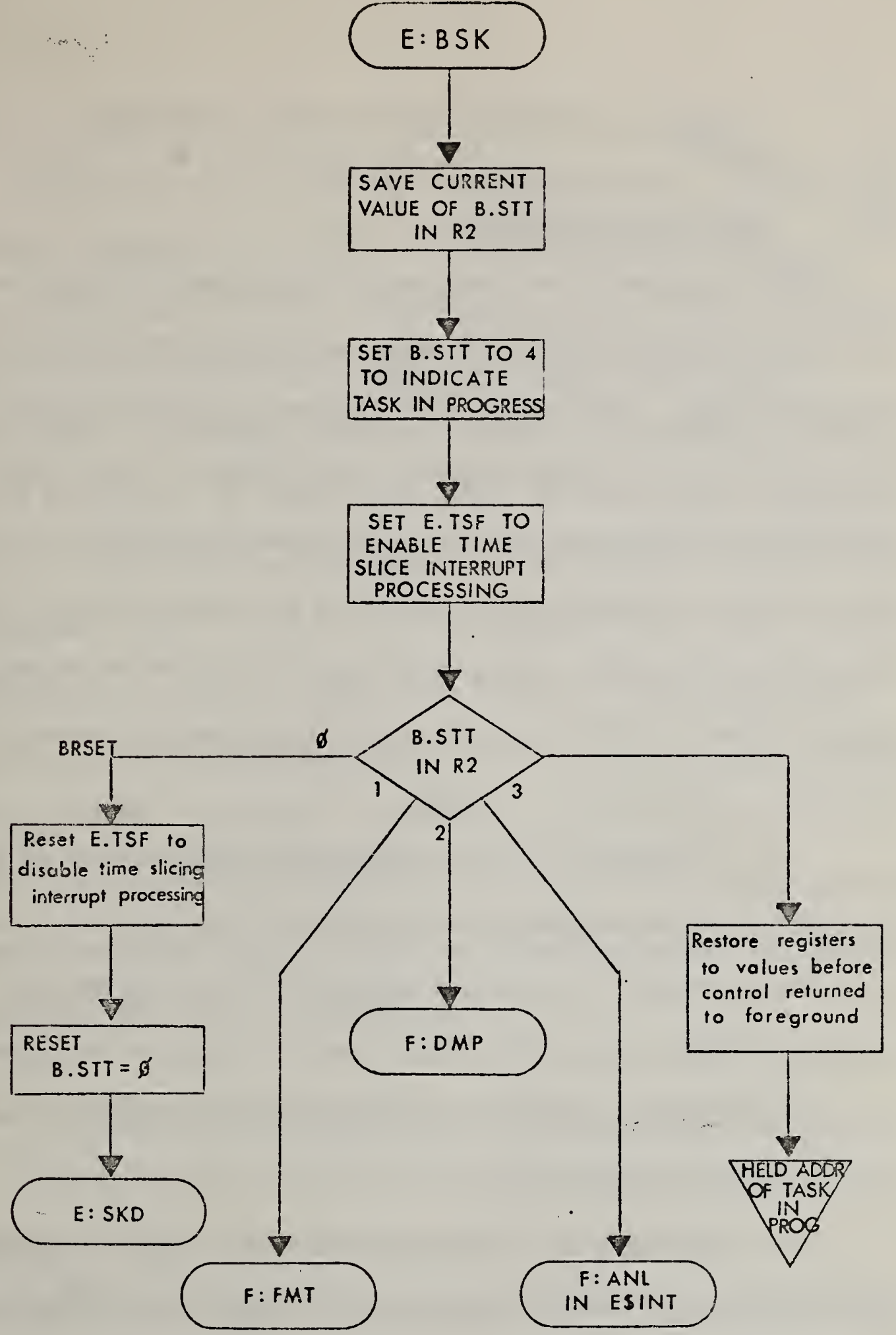


4. E\$TSI。

4.1 Functional Description。

E\$TSI contains the two programs which permit programs to operate on the background level while not inhibiting the processing of real-time tasks, Since background tasks will generally require some IO support and execute for relatively long periods of time, ROS must ensure that a background task not be permitted to hold the CPU to the point where priority tasks executed in the foreground are delayed from timely execution. Background tasks will therefore relinquish control to the real time tasks via the system scheduler in one of two ways:

a. Voluntarily。 When a background task requires an IO operation (such as additional information from the operator, or data from the mag tape), the program will make its request through B:IOR (in E\$TSI), which will initiate the IO through the necessary peripheral handler, and then return control to the foreground until that IO action is completed.

b. Involuntarily。 If a background task is executing when the time slicing interrupt ozcurs (ten per second), E:TSI returns control to the foreground until any pending real-time tasks are serviced. 


\subsubsection{E:TSI (Time Slicing Interrupt Service Routine)。}

E:TSI is entered for all time slicing interrupts, It determines whether a background task is executing or not by the status of the time slicing flag (E.TSF)。 If the flag is not set, then the interrupt occurred during other than a background task, and control is immediately returned to whatever program was executing at the time of the interrupt. If, on the other hand, the flag is set, a background task was interrupted and the contents of all registers available to the background task (1 through 7 and $1 \varnothing$ through 15 ) are saved, as is the address of the next instruction to be executed in the background program. The time slicing flag is reset to show that control is being returned to the foreground, and E:BSK is rescheduled in the scheduler's task table to continue processing of the background program when the priority 15 task is ready to be serviced again, Control is returned to the foreground at E:SKD。

\subsubsection{B:IOR (Background Program IO Request Routine).}

When a background program requests an IO action from the operating system, that request is made through B:IOR, which coordinates that request with real-time requirements, The current register contents and the location counter are saved to permit execution to be continued at the next location following the call to 
B:IOR in the background program when the IO transaction is completed. The time slicing flag is reset prior to entry to any of the operating system's peripheral handlers to prevent possible confusion by Sibsequent entry to that same program in response to a real-time requesto The calling sequence supplies a parameter word which specifies whether the request is ?or the mag tape or the ASR 35 handler. Additional parameters associated with the particular device specified are then transferred to that device's handler. When control is returned, B:IOR transfers it to the foreground at E:SKD. When the IO transfer is complete, flag bits in the parameter word signal the hanjler to reschedule E:BSK, which will continue execution of the background task with the data from the transfer ready for processing.

The calling sequence for the two devices differ。 If input is required from the mag tape unit, the following sequence is used:

$\begin{array}{ll}\text { BLM, } 15 & \text { B:IOR } \\ \text { DFC } & \text { \#2 } \varnothing \varnothing \varnothing \quad \text { BIT 2 SPECIFIES MAG TAPE INPUT } \\ \text { DFC } & \text { (message start address) } \\ \text { DFC } & \text { (count of words to be read) }\end{array}$

IO communication batween the background program and the operator via the ASR 35 teletype is more variable. In this case the calling sequence is: 
BLM, 15

B:IOR

DFC

(parameter word)

DFC

(message start address)

where the parameter word is interpreted as follows:

BIT $\varnothing=\varnothing \quad$ Do not reschedule E:BSK immediately after the output is completed, This option is normally used with BIT $1=1$ to permit the background to request information from the operator, then not reenter the background program until the operator's response has been read.

$=1$ Reschedule E:BSK as soon as the output message specified by the second parameter word has been completed,

BIT $1=\varnothing \quad$ No response to the program's output is expected from the operator。

$=1$ The message specified by the second parameter word is a request for information from the operator. Do not reschedule E:BSK until that response is completed.

BIT $2=\not D \quad$ Specifies request for action on the ASR 35 。

$=1$ Used to specify mag tape IO action。

BIT $3=1 \quad$ Must be set as B:IOR teletype actions must begin with an output action,

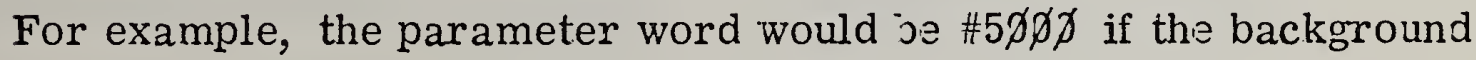
program were requesting information from the operator. The background program would then continue execution after the operator response was completed, If the background program needed to print

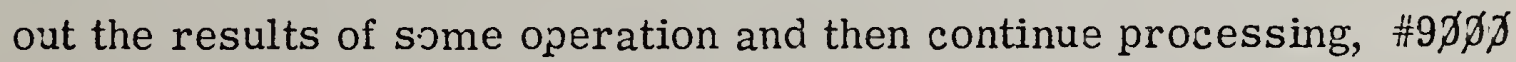
would be used as the parameter word, 
4.2 Called Subroutines.

Sibroutine Program Function

E:035 E\$T35 Transfers oatput message to buffer before transmission to tha ASR 35 。

Also sets flag bits to control ressheduling $\mathrm{E}$ :BSK based on value of $\mathrm{E}$.IOF。

E:MGS E\$MAG Initiates Mag Tape read into specified area of memory。

4.3 Input Parameter Deseriptions.

Parameter Prozram Deseription

E。TSF E\$SKD

Time slicing flaz; (see section 4,1)

BIT $\varnothing=\varnothing$ to inhibit processing of time slice interrupt

$=1$ to permit processing of time slice inter rupt

B. RS1 E\$SKD Holding buffer for registers 1-7 of interrupted background task.

B。 RS2 E\$SKD

Holding buffer for registers $1 \varnothing-15$ of interrupted background task.

B. RET E\$SKD Return lozation for interrupted background task.

E. T15 E\$SKD Task table entry for background system (E:BSK)。

E.SKD E\$SKD Scheduler reentry pointo

E. IOF E $\$$ T35 Parameter word used in calling E:035。 
4.4 Output Parameter Descriptions,

$\underline{\text { Parameter Size } \quad \text { Description }}$

E:TSI

Entry to time slicing interrupt routine

B:IOR

Entry to background IO request routine 

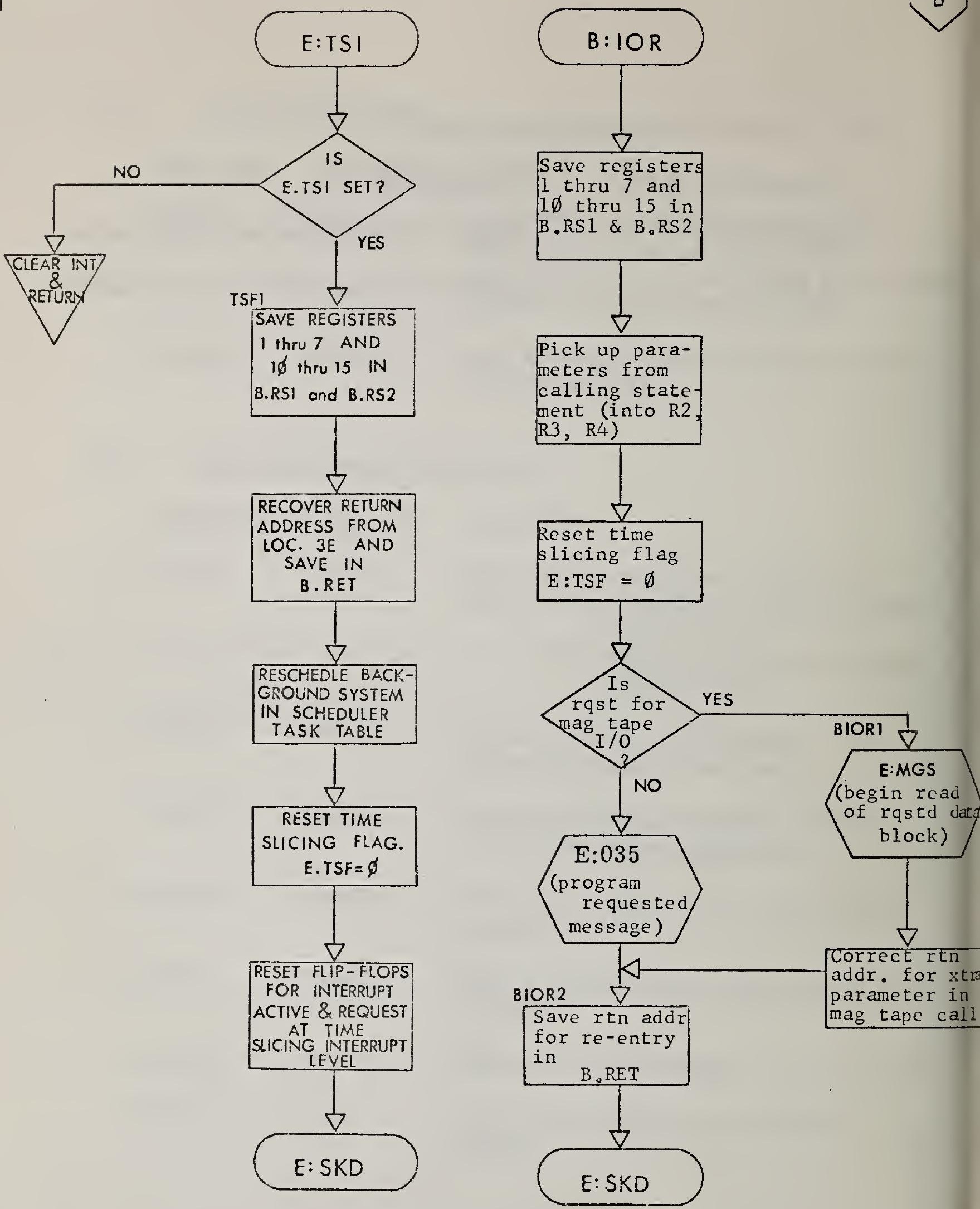

5 。 $E \$ C L K$
5.1 Functional Description

E\$CLK is the NUSTAD software clock. It contains the service routines for the one per second (E:SEC) and one per minute (E:MIN) time pulses from the system World Time Clock (WTC)。

\subsubsection{E:SEC (One Second Timing Interr upt Service Routine)。}

The one second time pulse triggers the level 1 external interrupt of the IOIS, Control is vectored to E:SEC, the service routine for that interrupt. It performs the following functions:

a. Supply a pulse to the ADACS power supply detection circuitry。 If twenty seconds elapses without this puise being received by the detectors, the power is turned off to the ADACS units and the sensors revert to their manual settings.

b. Check the delay times for scheduling M\$ESC. When a change occurs in the sensor status which results in an INE interrupt, it takes several milliseconds for the levels to stabilize before meaningful values can be read, This may result in numerous interrupts, most with meaningless value changes associated. Also, when the operator changes the sensor frequency, interrupts would occur for each digit changed, NUSTAD thercfore resets a five second delay timer with each INE interrupt。 When that timer (M.TM1) runs 
out, M\$ESC is scheduled in the system scheduler。

c. Initiate a data transfer of $U$ data from the sensors via the AIS DMP。After initiating this transfer, E:SEC checks to ensure that the DMP is then in use. If it isn't, NUSTAD will log a failure in the AIS (message reading " $T$ AF"), and set a flag to inhibit repetition of this message until a good transfer is initiated。 Without the AIS operating, E\$UIN will not be executed and real-time U data processing will cease。

\subsubsection{E:MIN (One Minute Timing Interrupt Service Routine)。}

The one minute time pulse triggers the level 2 external interrupt of the IOIS。 Control is vectored to E:MIN, the service routine for that interrupto It performs the following functions:

a. Check the timer for rescheduling U\$RED, the channel redundancy checker。 U. TM1 is a thirty minute timer which is incremented in E:MIN each minute until it reaches zero. When that happens, it is reset and U\$RED is scheduled in the system scheduler。

b. Data are saved to determine the following historical information:

1. Percentage of time each channel is in the UP state.

2. Percentage of UP time that each channel was at each of the possible TRACKING RATE settings。 
3. Percentage of UP time that each channel was in each RF GAIN grouping。

c. Read the BCD time to determine whether it is time to schedule execution of the M\$STD or M\$LEV programs。

\subsection{Called Subroutines。}

$\begin{array}{lll}\text { Subroutine } & \text { Program } & \text { Function } \\ \text { E:LOG } & \text { E\$LOG } & \begin{array}{l}\text { To generate coded messages for the } \\ \text { station log and initiate their transfer } \\ \text { to the ASR 28 via E\$T28. }\end{array}\end{array}$

5. 3 Data Formats.

The information needed by M\$LEV in generating historical data tables to evaluate channel reliability is stored in the following areas:

a. E. TM3 counts the minutes that the system has been on-line since the last execution of M\$LEV or since the last restart, whichever is more recent。

b. M. UPT is a series of counters, one per channel, used to count the minutes during which the channel was in an UP state.

c. M. TRT is a set of counters, with ten (one per tracking rate value) for each channel, used to tally the current tracking rate value each minute.

do M. GNT is a set of counters, with eight for each channel, used to tally the gain group in which the current gain is found each 
minute. The relation between the individual gain settings and the gain groups is given in the table below:

\begin{tabular}{|c|l|}
\hline GAIN GROUP & RF GAIN VALUES \\
\hline$\emptyset$ & $\emptyset, 1,2$ \\
1 & $3,4,5$ \\
2 & $6,7,8$ \\
3 & $9,1 \varnothing, 11$ \\
4 & $12,13,14$ \\
5 & $15,16,17$ \\
6 & $18,19,2 \varnothing$ \\
7 & $21,22,23,24$ \\
\hline
\end{tabular}

\section{TABLE 5.1}

THE RELATION BETWEEN GAIN SETTINGS AND GAIN GROUPS

The organization of the counters used to tally gain and tracking rate settings is graphically represented in Table 5.2 。

The times at which M\$STD and M\$LEV are automatically scheduled are maintained in STDTB and LEVTB respectively。 These tables allow the user to designate up to six times per day for M\$LEV and up to twenty four times per day for M\$STD to be automatically executed. The format in which the time is stored is given in Table 5.3 , and the current times now included in the system are given in Table 5.4。 


\begin{tabular}{|c|c|c|}
\hline CHANNEL & $\begin{array}{l}\text { TRACK } \\
\text { RATE }\end{array}$ & $\begin{array}{l}\text { MEMORY } \\
\text { LOCATION }\end{array}$ \\
\hline A & $\begin{array}{l}\varnothing \\
1 \\
2 \\
3 \\
4 \\
5 \\
6 \\
7 \\
8 \\
9\end{array}$ & $\begin{array}{l}\text { M. TRT } \\
\text { M. TRT+1 } \\
\text { M. TRT+2 } \\
\text { M. TRT+3 } \\
\text { M. TRT }+4 \\
\text { M. TRT+5 } \\
\text { M. TRT+6 } \\
\text { M. TRT }+7 \\
\text { M. TRT+8 } \\
\text { M. TRT+9 }\end{array}$ \\
\hline$\overline{\mathrm{B}}$ & $\begin{array}{l}\varnothing \\
1 \\
2 \\
3 \\
4 \\
5\end{array}$ & $\begin{array}{l}\mathrm{M}_{0} \mathrm{TRT}+1 \varnothing \\
\mathrm{M}_{0} \mathrm{TRT}+11 \\
\mathrm{M}_{0} \mathrm{TRT}+12 \\
\mathrm{M}_{0} \mathrm{TRT}+13 \\
\mathrm{M}_{0} \mathrm{TRT}+14 \\
\mathrm{M}_{0} \mathrm{TRT}+15\end{array}$ \\
\hline & $\zeta$ & $\xi$ \\
\hline $\mathrm{N}$ & $\begin{array}{l}7 \\
8 \\
9 \\
\end{array}$ & $\begin{array}{l}\text { M. TRT }+137 \\
\text { M.TRT }+138 \\
\text { M.TRT }+139\end{array}$ \\
\hline
\end{tabular}

\begin{tabular}{|c|c|c|}
\hline CHANNEL & $\begin{array}{r}\text { GAIN } \\
\text { GROUP } \\
\end{array}$ & $\begin{array}{l}\text { MEMORY } \\
\text { LOCA TION }\end{array}$ \\
\hline A & $\begin{array}{l}\varnothing \\
1 \\
2 \\
3 \\
4 \\
5 \\
6 \\
7\end{array}$ & $\begin{array}{l}\text { M.GNT } \\
\text { M. GNT+1 } \\
\text { M。GNT+2 } \\
\text { M.GNT+3 } \\
\text { M.GNT+4 } \\
\text { M。GNT+5 } \\
\text { M. GNT+6 } \\
\text { M.GNT+7 }\end{array}$ \\
\hline $\bar{B}$ & $\begin{array}{l}\varnothing \\
1 \\
2 \\
3 \\
\end{array}$ & $\begin{array}{l}\text { M。GNT }+8 \\
\text { M.GNT }+9 \\
\text { M.GNT }+1 \emptyset \\
\text { M.GNT }+11\end{array}$ \\
\hline & & 5 \\
\hline $\mathrm{N}$ & $\begin{array}{l}5 \\
6 \\
7\end{array}$ & $\begin{array}{l}\text { M. GNT }+1 \varnothing 9 \\
\text { M.GNT }+11 \emptyset \\
\text { M. GNT }+111\end{array}$ \\
\hline
\end{tabular}

\section{GAIN TABLE \\ (M. GNT)}

TRACKING RATE TABLE

(M. TRT)

TABLE 5.2

M. TRT and M。GNT STORAGE FORMAT 


\begin{tabular}{|c|c|c|c|c|c|}
\hline Time of Day & Table Value & Time of Day & Table Value & Time of Day & Table Vallu \\
\hline$\varnothing \varnothing \varnothing \varnothing$ & $\varnothing \varnothing \varnothing \phi_{16}$ & $\varnothing 8 \varnothing \varnothing$ & $1 \varnothing \varnothing \varnothing_{16}$ & $16 \varnothing \emptyset$ & $2 \mathrm{C} \phi \phi_{16}$ \\
\hline$\varnothing 1 \varnothing \varnothing$ & $\varnothing 2 \varnothing \phi_{16}$ & $\varnothing 9 \varnothing \varnothing$ & $12 \varnothing \varnothing_{16}$ & $17 \varnothing \varnothing$ & $2 E \varnothing \emptyset_{16}$ \\
\hline$\varnothing 2 \varnothing \varnothing$ & $\emptyset 4 \varnothing \emptyset_{16}$ & $1 \varnothing \varnothing \varnothing$ & $2 \varnothing \varnothing \emptyset_{16}$ & $18 \phi \emptyset$. & $3 \not \phi \phi_{16}$ \\
\hline$\varnothing 3 \varnothing \varnothing$ & $\emptyset 6 \varnothing \emptyset_{16}$ & $11 \varnothing \varnothing$ & $22 \varnothing \emptyset_{16}$ & $19 \varnothing \varnothing$ & $32 \varnothing \phi_{16}$ \\
\hline$\varnothing 4 \varnothing \varnothing$ & $\varnothing 8 \varnothing \emptyset_{16}$ & $12 \varnothing \varnothing$ & $24 \varnothing \emptyset_{16}$ & $2 \varnothing \varnothing \emptyset$ & $4 \varnothing \varnothing \emptyset_{16}$ \\
\hline$\varnothing 5 \varnothing \emptyset^{\circ}$ & $\varnothing \mathrm{A} \phi_{16}$ & $13 \varnothing \varnothing$ & $26 \varnothing \emptyset_{16}$ & $21 \varnothing \emptyset$ & $42 \varnothing \emptyset_{16}$ \\
\hline$\emptyset 6 \varnothing \emptyset$ & $\varnothing \mathrm{C} \varnothing \varnothing_{16}$ & $14 \varnothing \varnothing$ & $28 \varnothing \emptyset_{16}$ & $22 \varnothing \emptyset$ & $44 \phi \phi_{16}$ \\
\hline$\varnothing 7 \varnothing \emptyset$ & $\emptyset \mathrm{E} \varnothing \emptyset_{16}$ & $15 \varnothing \varnothing$ & $2 A \emptyset \emptyset_{16}$ & $23 \varnothing \varnothing$ & $46 \varnothing \emptyset_{16}$ \\
\hline
\end{tabular}

NOTE: To get times other than the even hours, add the minutes directly to the hexadecimal table value. For example: to get $173 \emptyset$, add $3 \varnothing$ to $2 \mathrm{E} \emptyset \emptyset 16$ to get a table value of $2 E 3 \varnothing_{16^{\circ}}$

TABLE 5.3

Time of Day to STDTB/LEVTB Table Entry Value Conversions 


\begin{tabular}{|c|c|}
\hline TOD & TOD \\
\hline$\varnothing 2 \varnothing \emptyset$ & $\phi \emptyset \emptyset \emptyset$ \\
\hline$\emptyset 6 \varnothing \emptyset$ & $\emptyset 8 \emptyset \emptyset$ \\
\hline $1 \varnothing \emptyset \emptyset$ & $16 \varnothing \emptyset$ \\
\hline $14 \varnothing \emptyset$ & (three entries unused) \\
\hline $18 \emptyset \emptyset$ & \\
\hline $22 \varnothing \emptyset$ & \\
\hline (eighteen entries unused) & \\
\hline
\end{tabular}

TABLE 5.4

CURRENT TABLE VALUES FOR STDTB AND LEVTB 

Input Parameter Descriptions.

\begin{tabular}{|c|c|c|}
\hline Parameter & Program & Description \\
\hline M。TM1 & MSESC & $\begin{array}{l}\text { Five second delay timer for } \mathrm{sc} \\
\mathrm{M} \$ \mathrm{ESC}\end{array}$ \\
\hline E. TØ2 & E\$SKD & Task table entry for M\$ESC \\
\hline E. TØ4 & E\$SKD & Task table entry for U\$RED \\
\hline E. Tø8 & E\$SKD & Task table entry for M\$STD \\
\hline E。T14 & E\$SKD & Task table entry for M\$LEV \\
\hline U. TRK & U\$DAT & Current tracking rate settings \\
\hline $\mathrm{U}_{0} \mathrm{GAN}$ & U\$DAT & Current gain settings \\
\hline
\end{tabular}

5. 5 Output Parameter Descriptions。

Parameter Size Description

E:SEC

E:MIN

U. TM1

M.TRT

M。 GNT

M. UPT

E.TM3
Entry to one second interrupt service routine Entry to one minute interrupt service routine

1 Timer for scheduling U\$RED

$14 \varnothing$

Table of tracking rate setting frequency (See Table 5。2)

112 Table of gain grouping frequency (See Table 5.2)

14 Table of channel up time frequency (See Section 5.3)

1 Counter for system up time (See section 5.3 ) 
5.6 Internal Data Descriptions.

Parameter Size Description

$\begin{array}{lll}\text { SAVRS } & 7 & \text { Register holding buffer }\end{array}$

$\begin{array}{lll}\text { SAVRR } & 7 & \text { Register holding buffer }\end{array}$

SFLAG $\quad 1 \quad$ Flag set to suppress repeated "AF" messages

LEVTB $6 \quad 6 \quad$ Table of times to schedule M\$LEV (See Table 5。4)

STDTB $24 \quad$ Table of times to schedule M\$STD (See Table 5.4) 


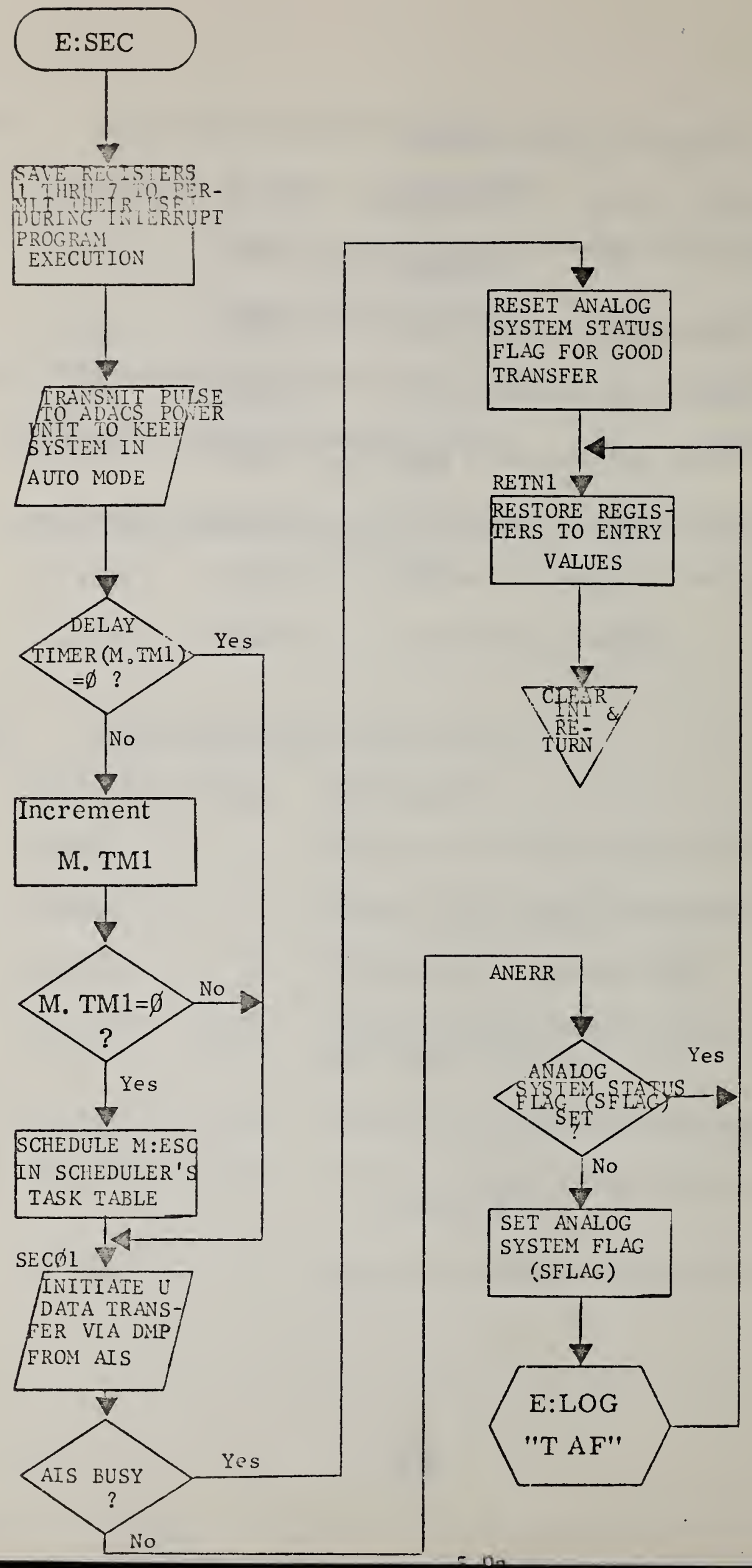




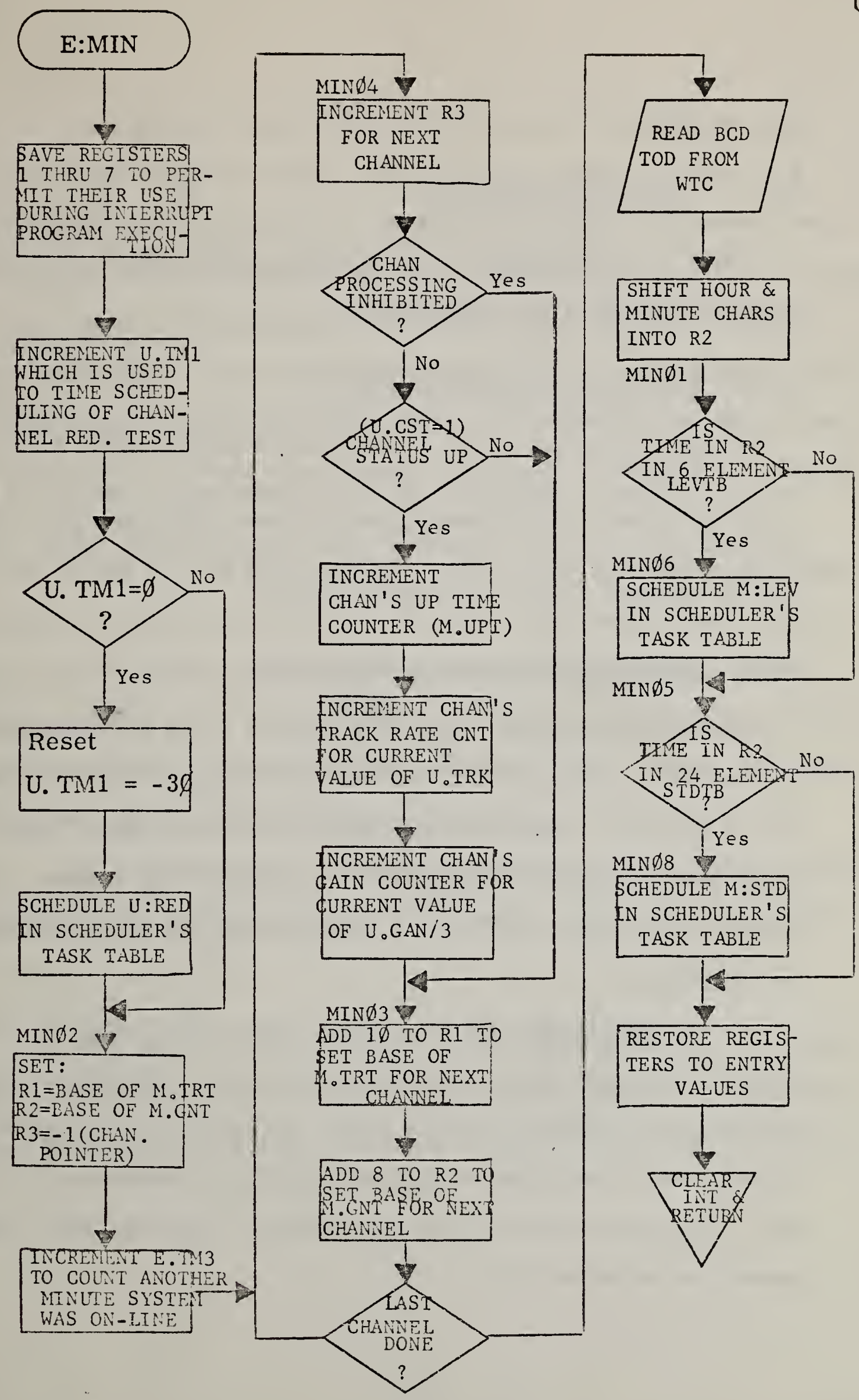

$5.9 b$ 
6. E\$UIN

6.1 Functional Description

E\$UIN is a preprocessor for the analog $U$ data, called after each transfer from the AIS is complete.

\subsubsection{E:ANL (AIS SI Service Routine)}

A service interrupt (SI) is generated by the AIS after a data transfer from it via DMP is completed. This interrupt vectors control to $E$ :ANL in E\$UIN where E:UIN is flagged in the scheduler's task table. Control is then returned to the interrupted program.

\subsubsection{E:UIN (U Data Preprocessing Routine)}

When E:UIN is entered from the scheduler, E.UBU will contain 42 AIS channels of analog $U$ data, multiplexed through the AIS and read in via the DMP. These 42 AIS channels break down to three inputs (amplitude, phase, and blanking rate) for each of the fourteen sensor channels (see Figure 6.1). E:UIN then performs the following tasks on these data:

a. Amplitude: The AIS presets several flag bits (see Figure 6.2) in the input word. E:UIN strips these away. The analog range at the sensor should be $\emptyset$ to $+1 \varnothing$ volts. If the low end adjustment is slightly off, a negative voltage may actually be transmitted to the AIS. Since the programs expect only positive values as input, negative points are converted to $\emptyset$. 
b. Phase: Again E:UIN strips away the flag bits preset by the AIS and checks for negative input values, converting them to $\emptyset$. The sensor has a hardware $\emptyset$ to $1 \emptyset \emptyset$ to $\emptyset$ equivalence. To prevent confusion in detecting when this shift of reference occurs, E:UIN anticipates it and sends a 50 microsecond phase shift to the reference frequency in the sensor mixer. This creates an apparent 50 microsecond shift in the phase input, toward the midrange value. To compensate for this processor induced shift in later computations, the sample is flagged to indicate that the shift will have occurred before the next sample is read. U.CYC is set to \pm 50 microseconds, a value which is added to subsequent samples to provide continuity to the phase input data stream. Since the maximum response of the sensor is about $15 \mathrm{microseconds} / \mathrm{second}$, the equivalent of 15 and 85 microseconds are used as the points at which the cycle shift is transmitted to the sensor. This prevents the sensor from ever reaching its automatic recycle point.

c. Blanking Rate: As with the amplitude value, the bits preset by the AIS are stripped away and the negative value to zero conversion is made.

As each value in E.UBU is examined as described above, it is moved in a holding buffer called E.UBF. When all 42 words of AIS input have been preporcessed and movec to E.UBF, two digital words of DOY/TOD data 
are read from the WTC via the IOIS and stored in the next two words of E.UBF. This associates a time value with the current data values. Forty-four words of E.UBF are therefore filled each time E:UIN is executed; i.e., once per second. After ten seconds, or $44 \emptyset$ words of E.UBF, the data are transferred to U.WRK, a working buffer, and the analysis programs are scheduled in E:SKD. The pointers for E.UBF are reset and it becomes available for the next ten seconds of data.

Actually, U.WRK is an eleven row buffer. After each ten seconds, E:UIN moves E.UBF to rows 2 through 11 of it. U\$RTA will have moved the previous eleventh row to the first so that the data from the most previous second prior to the current ten second period is also available for the analysis programs. During system startup, this first row of U.WRK must be filled by E:UIN with unprocessed input data. This startup state is identified by E.SKI being set, which signals the program to reset E.SFI and move the first row of E.UBF to the first row of U.WRK and then reset the pointers to begin normal data gathering, ten seconds at a time, for rows 2 through 11 of U.WRK.

\subsubsection{E:MIT (IOIS Digital Output Routine)}

E:MIT is an executive support routine which transmits the current tracking rate, gain, and blanking level settings to the desired sensor channel. It also transmits the 50 microsecond phase shift from E:UIN when needed. 
The calling sequence is:

$$
\text { BLM, } 5 \text { E:MIT }
$$

with the desired channel in R3. The tracking rate value is taken from the channel's U.TRK, the gain from U.GAN, the blanking level from U.BLL and the cycle shift bit from SHIFT. The format of the output word is shown in Figure 6.3. Only register 5 is affected by this routine.

\begin{tabular}{|cll|}
\hline CHANNEL & \multicolumn{1}{c|}{ VALUE } & LOCATION \\
\hline$A$ & AMPLITUDE & E.UBU \\
& PHASE & E.UBU+1 \\
& BLANKING RATE & E.UBU+2 \\
& & \\
B & AMPLITUDE & E.UBU+3 \\
& PHASE & E.UBU+4 \\
& BLANKING RATE & E.UBU+5 \\
& AMPLITUDE & E.UBU+6 \\
C & PHASE & E.UBU+7 \\
& & \\
\hline B & BLANKING RATE & E.UBU+38 \\
\hline & BMPLITUDE & E.UBU+39 \\
\hline
\end{tabular}

Figure 6.1 Input Buffer (E.UBU) Format 


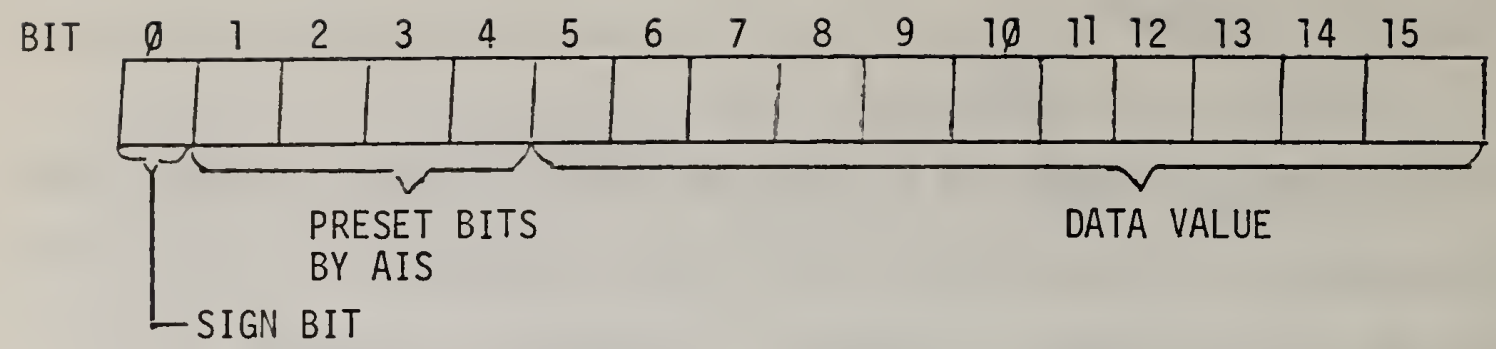

Figure 6.2 Input Data Word (AIS) Format

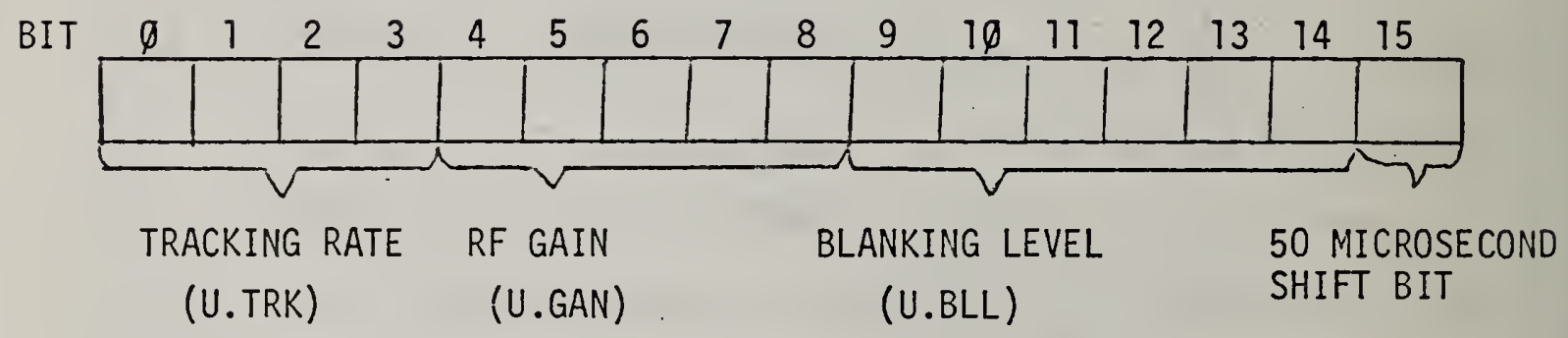

Figure 6.3 Output Data Word (IOIS) Format 
WORD $\emptyset$ OF IOIS EXTENDER:

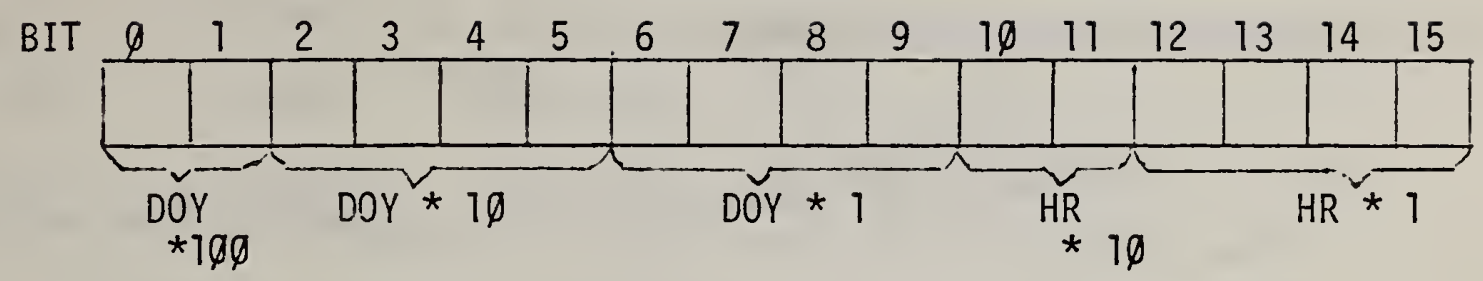

WORD I OF IOIS EXTENDER:

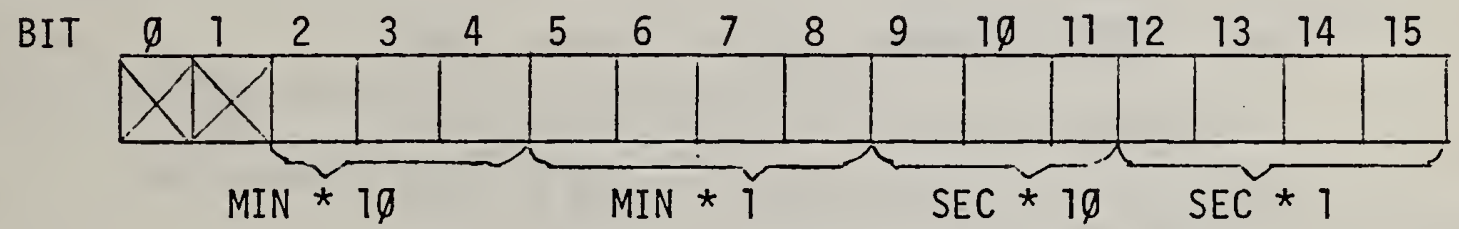

Figure 6.4 Input Data Word (IOIS EXTENDER WORDS $\emptyset$ ) Format

\subsection{Data Formats}

\subsubsection{AIS Input Data}

The analog data multiplex through the AIS supplies eleven bits plus sign for each word. Additionally, the AIS presets four bits to fixed values. See Figure 6.2 .

\subsubsection{Sensor Parameter Control Output via IOIS}

The tracking rate, RF gain, and blanking level settings are transmitted to the sensor/ADACS via words $\emptyset$ through 13 of digital output 


\subsubsection{DOY/TOD Input from the WTC via IOIS}

The DOY and TOD are read in from words $\varnothing$ and 1 of the channel extender for the digital input of the IOIS. See Figure 6.4.

\subsubsection{Amplitude Values}

The amplitude value ranges linearly over the range from zero to $20471 \varnothing$ corresponding to actual amplitude values from $-2 \emptyset \mathrm{dB}$ to $+2 \emptyset \mathrm{dB}$.

\subsubsection{Phase Values}

The phase values range linearly over the range from zero to 159910 corresponding to phase values from $\emptyset$ to 99.9 microseconds.

\subsubsection{Blanking Rate Values}

The blanking rate values range linearly over the range from zero to 204710 corresponding to blanking rates from $\emptyset \%$ to $10 \varnothing \%$.

\subsection{Input Parameter Descriptions}

PARAMEIER PROGRAM DESCRIPTION

$\begin{array}{lll}\text { U.IHB } & \text { U\$DAT } & \begin{array}{l}\text { Bit } \emptyset \text { set to inhibit processing on channel if INE } \\ \text { or Maintenance set on the channel. }\end{array} \\ \text { U.CYC } & \text { U\$DAT } & \begin{array}{l}\text { Set to } \pm 5 \emptyset \text { microseconds for channel when cycle } \\ \text { shift is sent to the sensor. }\end{array} \\ \text { U.WRK } & \text { U\$DAT } & \text { Working storage for data from sensor. } \\ \text { U.TRK } & \text { U\$DAT } & \text { Current tracking rate settings for the channels. } \\ \text { U.GAN } & \text { U\$DAT } & \text { Current RF gain settings for the channels. }\end{array}$




\subsection{Input Parameter Descriptions (Continued)}

PARAMETER PROGRAM DESCRIPTION

U.BLL U\$DAT

Current blanking level settings for the channels.

E.TØ1

$E \$ S K D$

Task table entry for R\$RTA

E.Tø3

$E \$ S K D$

Task table entry for E:UIN

\subsection{Output Parameter Descriptions}

\section{PARAMETER SIZE DESCRIPTION}

E:UIN Entry point for data preprocessor

E:ANL Entry point for AIS SI service routine

E:MIT Entry point for executive service routine to transmit sensor parameter settings to equipment.

E:UBU $\quad 42$ Input buffer for data read in from AIS via DMP

E.UBF $\quad 44 \varnothing \quad$ Holding buffer for input data, preprocessed with time words appended, for ten seconds.

E.SF1 1 Bit $\emptyset$ set for first AIS record, reset thereafter

6.5 Interna? Data Descriptions

PARAMETER SIZE DESCRIPTION

SHIFT

SCONT
Bit 15 used to $f 7 a g$ need to send 50 microsecond phase shift.

1 Ten second counter used to recognize when E.UBF is full. 


\section{ESUIN}

$E: A N L$

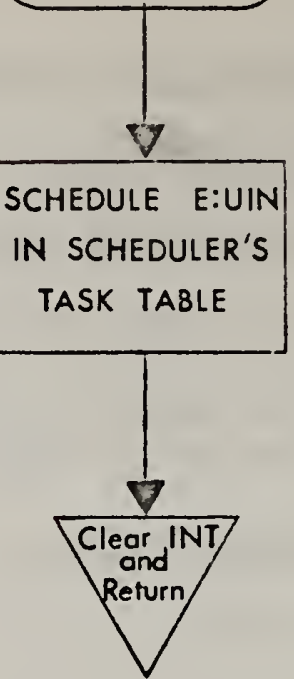

$6.8 a$ 

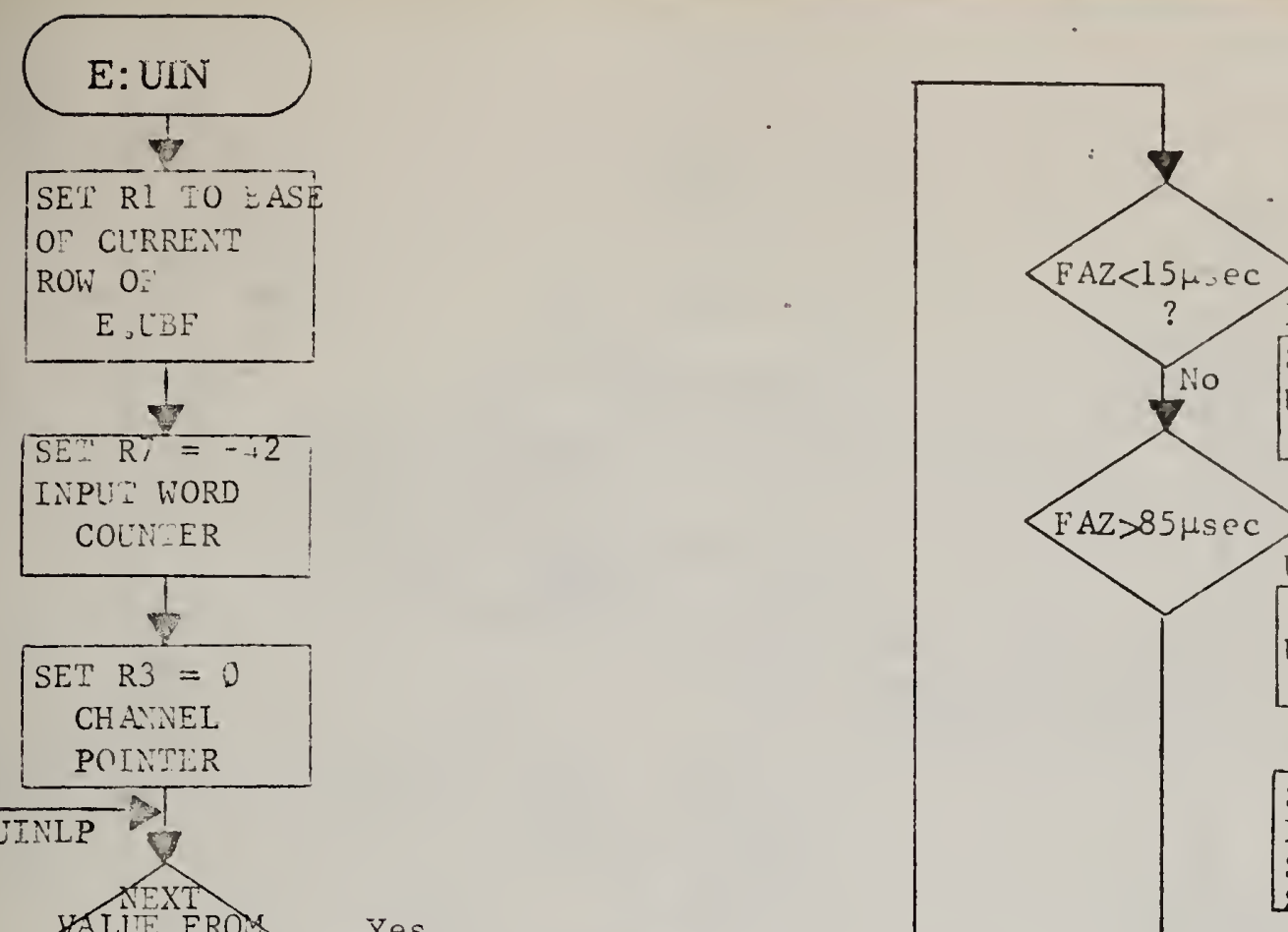

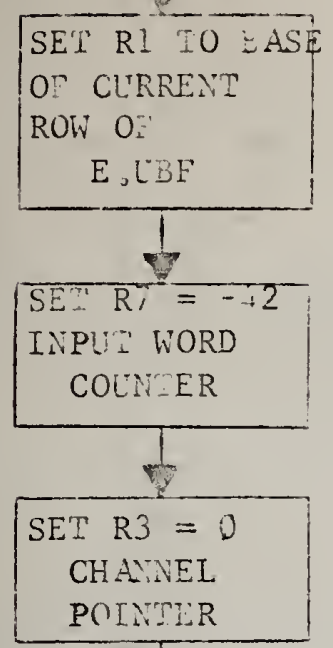

ASE OF CLRRENT

UINLP

ALUE FROM E.LUU(AIP) Yes

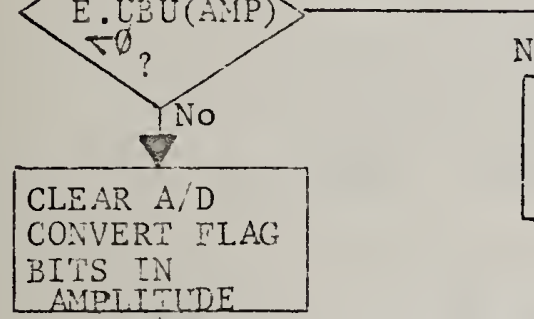
NEGAM SE_ AMPLIIUDE VAL:E -
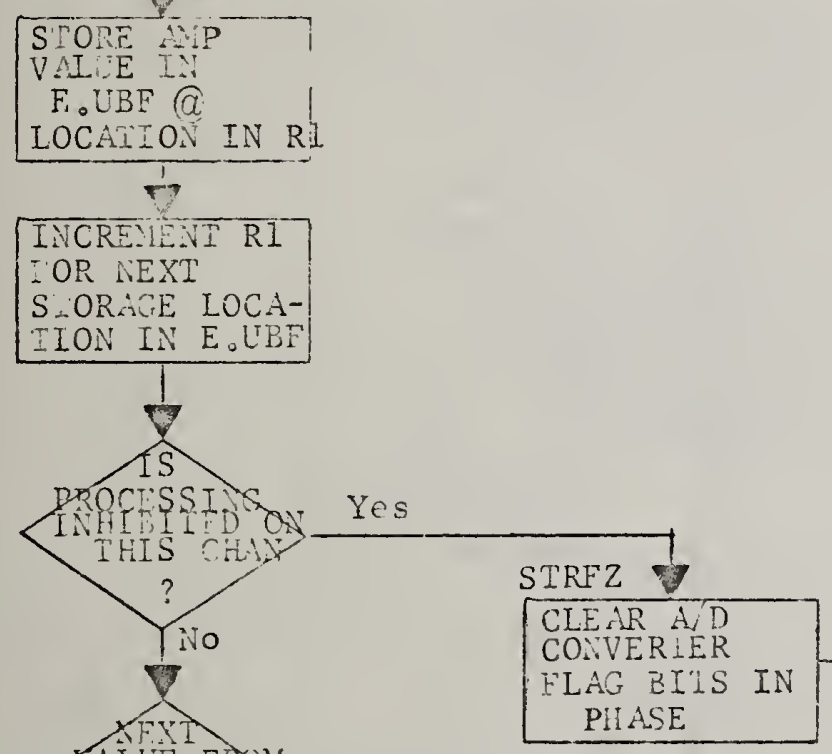

AALUE FोM Y

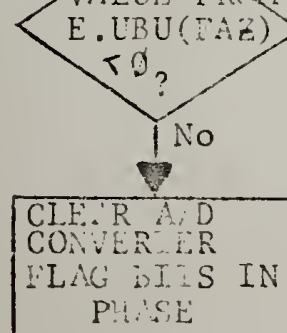

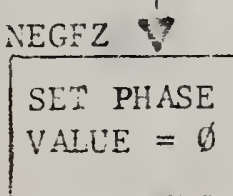

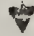

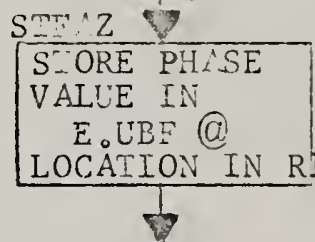

INCREIENTT RI FOR NEXI

STORAGE LOCA-

TION IN E。UBF

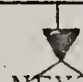

AEXN

TALHE ERP Y Y

RAT

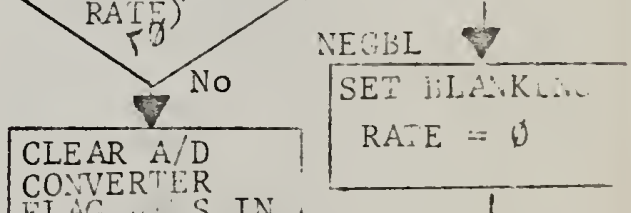

CLEAR A/D

EEATKT $\mathrm{S}_{\mathrm{RAN}} \mathrm{IN}$

$S E \cdots+N O D O$

P: SE VAL TE

$S E_{-1}^{\prime \prime} 50_{\mu} \ldots-C$

SHIFT $3 T \mathrm{Tm}$
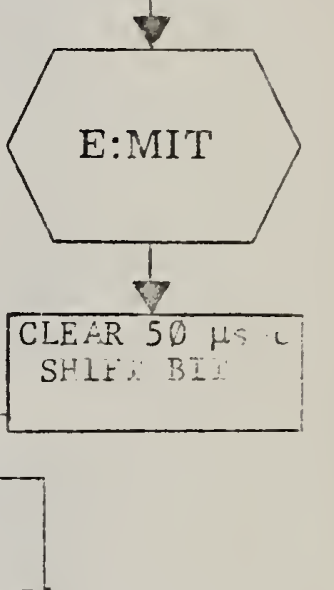

\section{.}




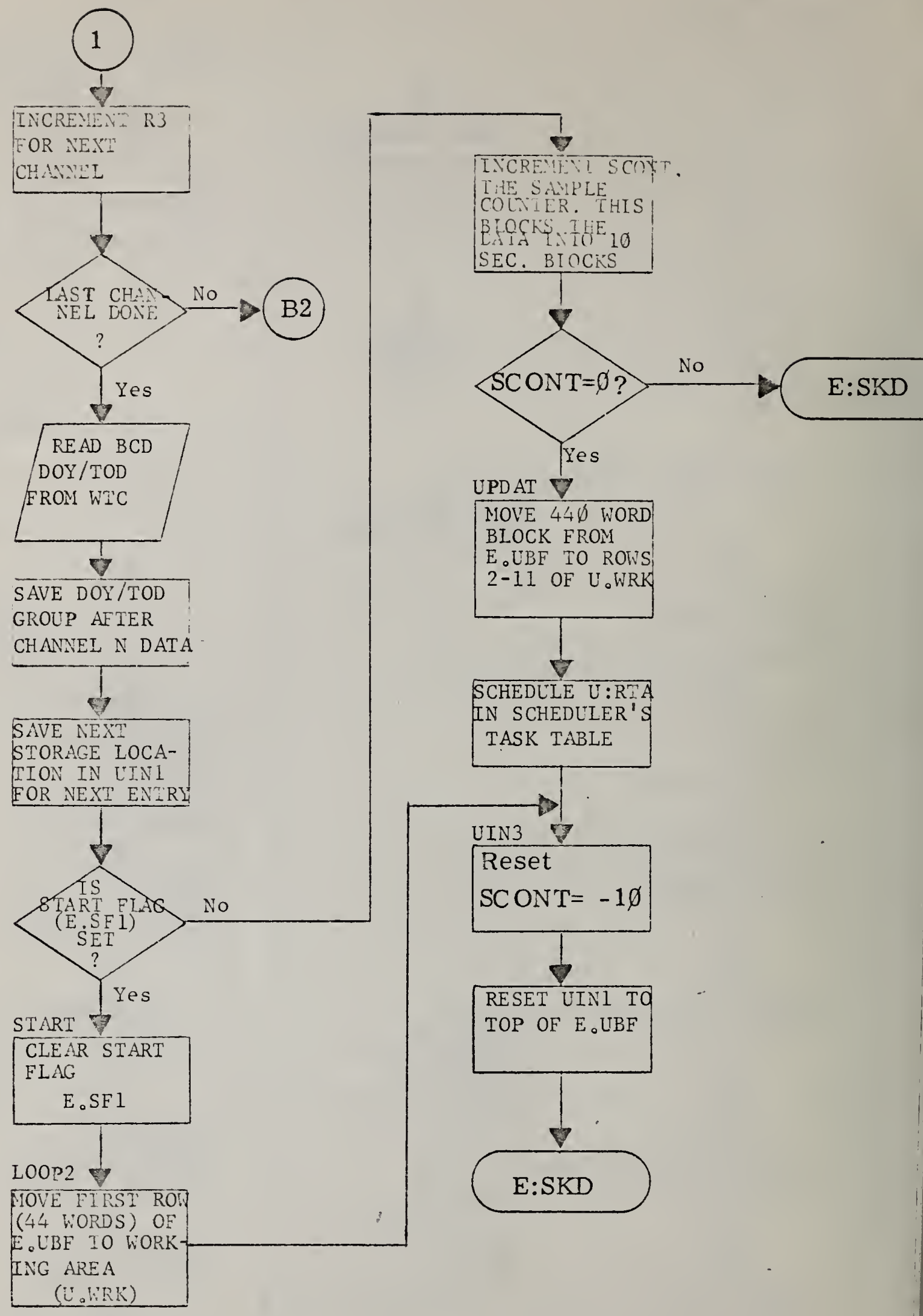




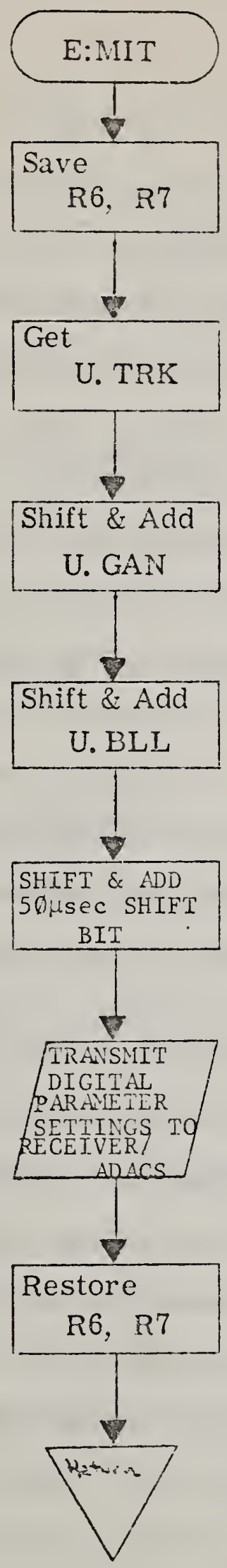


7. $\underline{E S U B}$

7.1 Functional Description

E\$SUB contains a set of interrupt service routines for power loss, memory parity errors, and console interrupts.

\subsubsection{E:PND (The Power Loss Interrupt)}

Hardware power sensors vector control to the power down subroutine (E:PWD) at least $2 \varnothing \emptyset$ machine cycles prior to total power failure. This program resets the interrupt location, which indicates where the power failure service routine is located, to point to the power up subroutine (E:PWU). When the power is restored, control will then be vectored to that program where processing may be resumed. E:PWD also reads the current $B C D$ time as the time of the power faiture. The program then hangs itself in a tight no-op loop until the power drops completely out.

\subsubsection{E:PWU (The Power Restored Interrupt)}

When power is restored to the processor, an interrupt is generated which vectors control to the power up subroutine, E:PWU. Here the current time is read to indicate when power was restored, and a message is generated to $\log$ the power loss and resumption. The interrupt location is reset to vector control to E:PWD for the next failure. Since any loss of continuity in data is considered serious, and the fact that power fluctuations causes noise spikes in the sensor, the system will be restarted after each power failure. Therefore, control is transferred to $E: A N F$ in $E \$ I N T$. 


\subsubsection{E:PAR (The Memory Parity Error Interrupt)}

When the hardware memory parity checking circuitry detects a memory parity error, control is vectored to E:PAR. This routine generates a $\log$ message indicating the time of the error detection and the memory location at which the error was detected. For the first two such errors, the system will restart at E:ANF in E\$INT. In this way, if the location affected is not executed often, the system will continue to process where it can. System restarts would serve no purpose if the error were common to many sections of memory, or if they were in heavily used sections of the program, so the system halts if more than two errors are detected.

\subsubsection{E:ABR (The Console Interrupt)}

When the operator depresses the Console Interrupt switch on the console panel, control is vectored to $E: A B R$, where $B: O R T$ is scheduled in the scheduler's task table. Control is then returned to the program in progress.

\subsubsection{B:ORT (The Background Task Abortion Routine)}

$B: O R T$ is entered from the scheduler in response to a console interrupt. This routine resets B.STT (the background status) to $\emptyset$, generates a message to the operator on the ASR 35 announcing, "BACKGROUND TASK ABORTED BY OPERATOR," and returns control to the scheduler at E:SKD. 
If the background scheduler should be entered again, it will find B.STT $=\varnothing$ and bypass background processing.

7.2 Called Subroutines

SUBROUTINE PROGRAM FUNCTION

E:FRT E\$FRT Reformats BCD time from the WTC to ASCII for output.

E:FCT E\$FRT Reformats BCD time from memory to ASCII for output.

E:028 E\$T28 Translates message to BAUDOT code and stores it in buffer before transmission to the ASR 28.

E:035 E\$T35 Transfers output message to buffer before transmission to the ASR 35.

7.3 Input Parameter Descriptions

PARAMETER PROGRAM DESCRIPTION

\begin{tabular}{|c|c|c|}
\hline $\begin{array}{l}E: T M 7 \\
E: T M 2\end{array}$ & E\$FRT & $\begin{array}{l}\text { Two word BCD time used as parameter value for } \\
E: F C T \text {. }\end{array}$ \\
\hline $\mathrm{E} .28 \mathrm{~F}$ & $E \$ T 28$ & Parameter word used in calling $E: 028$. \\
\hline E.IOF & $\mathrm{E} \$ \mathrm{~T} 35$ & Parameter word used in calling $E: 035$. \\
\hline B.ST & $E \$ S K D$ & Background processing status (See Table 3.2 ). \\
\hline E. $35 \emptyset$ & E\$T35 & ASR 35 I0 status word. \\
\hline E.TIØ & $E \$ S K D$ & Task table entry for B:ORT. \\
\hline$E: A N F$ & E\$INT & System Restart Point. \\
\hline$E: S K D$ & $E \$ S K D$ & Scheduler entry point. \\
\hline
\end{tabular}


7.4 Output Parameter Descriptions

PARAMETER SIZE DESCRIPTION

E:PWD Entry point to power down interrupt service routine.

$E: P A R \quad$ Entry point to memory parity interrupt service routine.

E:ABR Entry point to console interrupt service routine.

B:ORT Entry point to background task abortion routine.

7.5 Internal Data Descriptions

PARAMETER SIZE DESCRIPTION

PWDT1 2 Storage for BCD time of power down

PWRMS 11 Power failure message "123 123456 T PF"

PMS1 9 Power restored message "123 123456 T PR"

PARMS 15 Memory parity message "123 123456 T MP AT $\emptyset \emptyset \emptyset \emptyset "$

PARS1 Memory location in PARMS

PARCT 1 Counter for memory parity errors

BMSG $21 \quad$ Console Interrupt message:

"BACKGROUND TASK ABORTED BY OPERATOR." 

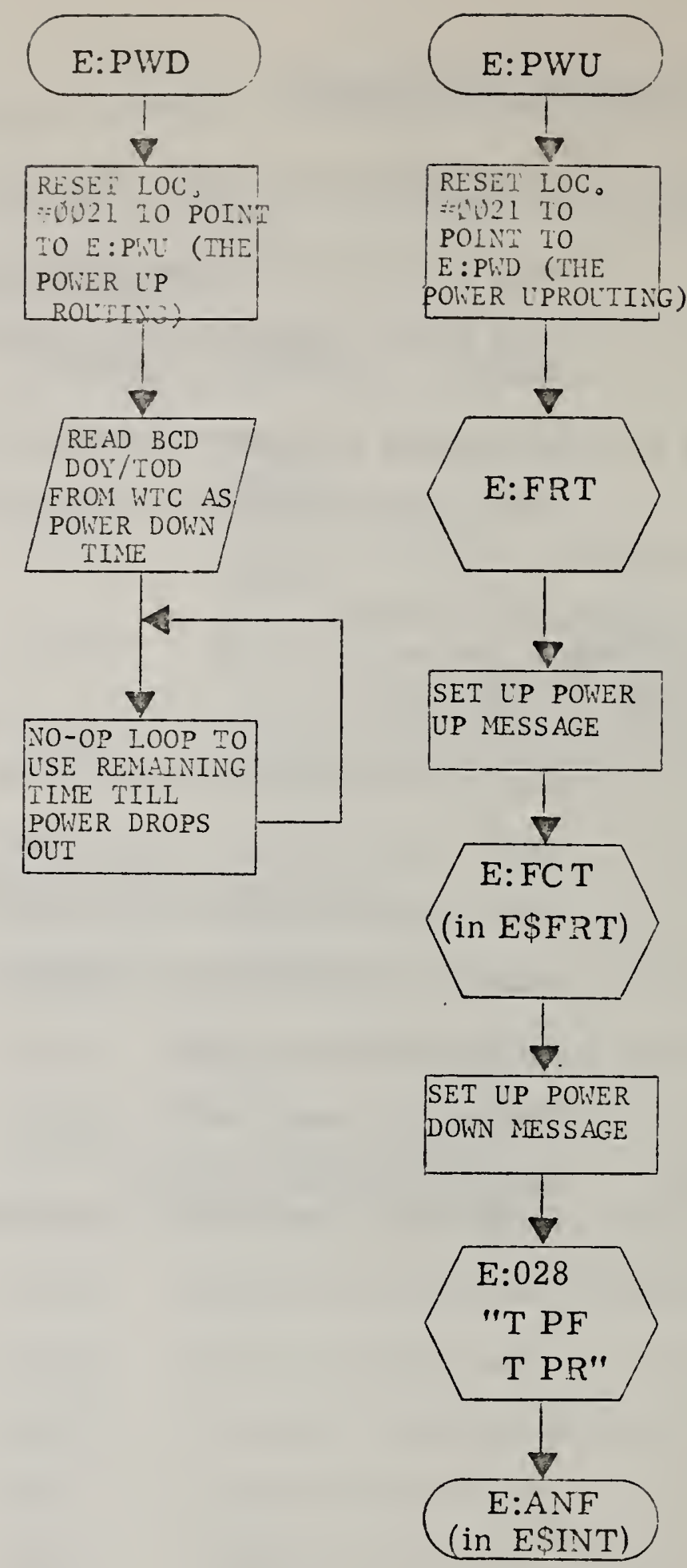
$E: P A R$

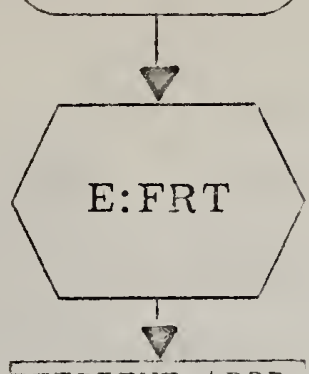

RETRIEVE ADDR.

AT WHICH PARITY

ERROR WAS EOLND

FROY LOC.:O0 23
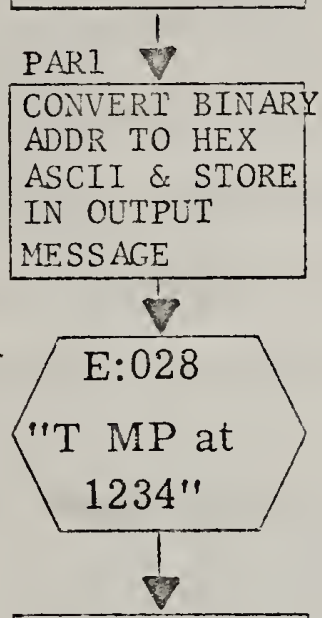

INCREMENT

ERROR COLNTER

(PARCT)

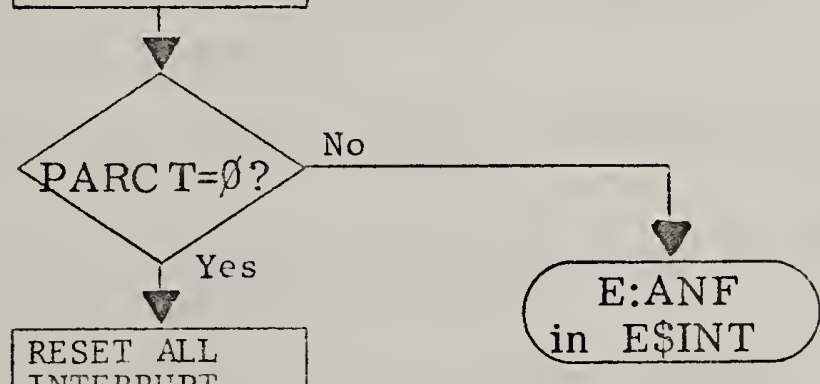

INTERRLPT

ENABIE FLIP-

FIOPS TO DIS-

ABIE INTRPIS。

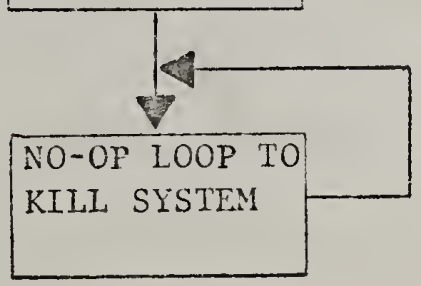




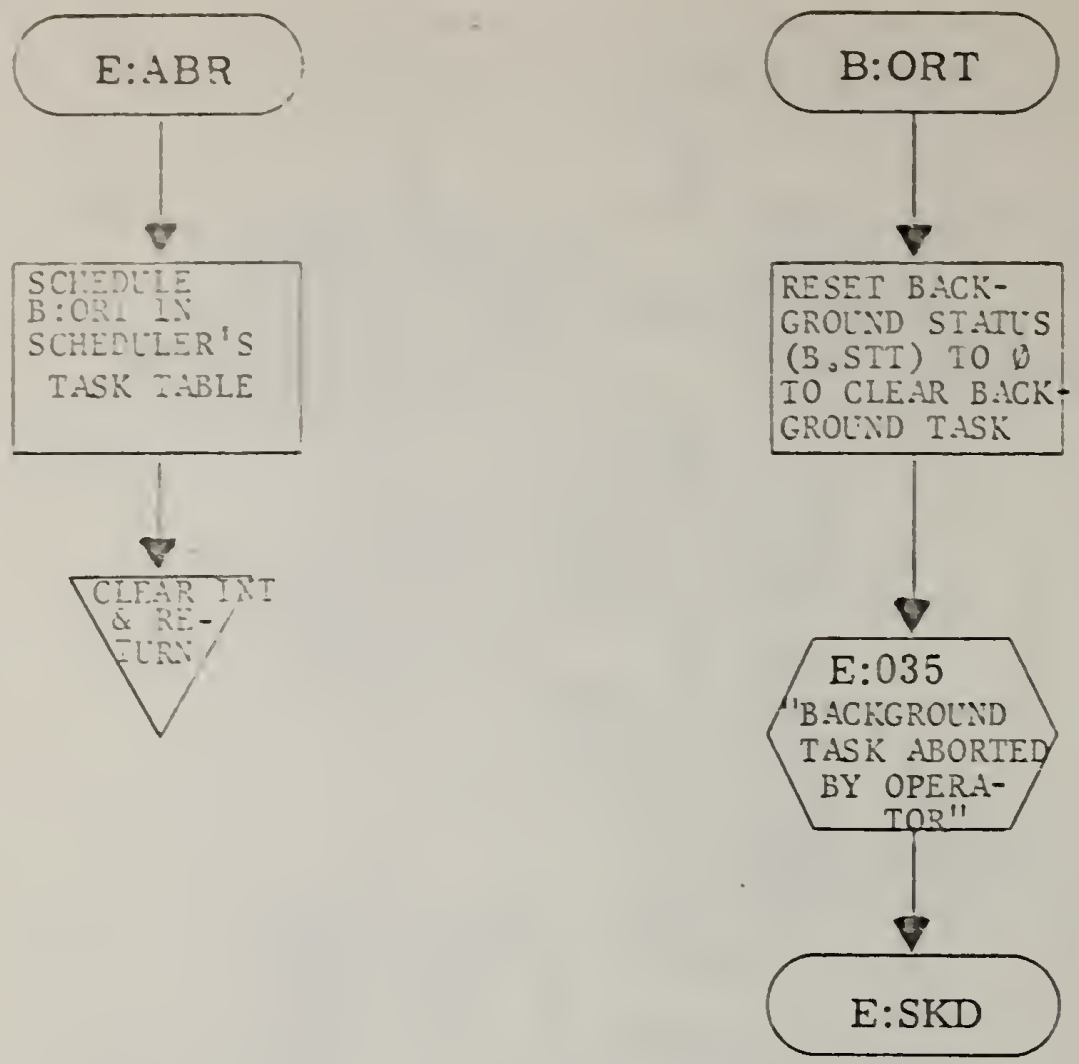




\section{E\$OMI}

\subsection{Functional Description}

When the ASR 35 is in the request mode (i.e., awaiting input not in response to a background task request for information), ESOMI wi 11 be scheduled when the operator completes his input, terminated by a carriage return character. Appendix III provides operating instructions on the operator's intercourse with the system. His request for system action is received by $E: O M I$ as a four character keyword and perhaps additional information required by the desired task. Table 8.1 lists the normal keywords and the actions they generate.

\begin{tabular}{|c|c|}
\hline KEYWORD & ACTION RESULTIIIG \\
\hline LOG: & Transfer operator's message to station $\log$ on ASR 28 \\
\hline DFC: & $\begin{array}{l}\text { Set B.STT }=1 \text {, schedule E:BSK to execute DRFUD tape } \\
\text { format checking routine. }\end{array}$ \\
\hline DDD: & $\begin{array}{l}\text { Set } B . S T T=2 \text {, schedule } E: B S K \text { to execute DRFUD data } \\
\text { display routine. }\end{array}$ \\
\hline DDA: & $\begin{array}{l}\text { Set B.STT }=3 \text {, schedule E:BSK. This now executes a } \\
\text { dummy task in E\$INT. }\end{array}$ \\
\hline OPC: & $\begin{array}{l}\text { Schedule U:PST to initiate operator parameter change to } \\
\text { a sensor channel. }\end{array}$ \\
\hline STD: & $\begin{array}{l}\text { Schedule M:STD to generate a Current Status Display } \\
\text { table in the station log. }\end{array}$ \\
\hline CRC: & Schedule U:RED to perform a channel redundancy test. \\
\hline
\end{tabular}

Table 8.1 E:OMI Keyword Decoding List 
If the first four characters of the operator's input does not match any of the keywords in the above list, bit 7 of the console switch register is checked. If it is set, two additional keywords are available; "DIS:" and "SET:". These additional options are protected by the position of bit 7 as they are intended for the use of programmers knowledgeable in the software system design, and not by the normal operator. The DIS: option cuases the contents of a specified memory location to be displayed on the ASR 35. The SET: option permits the operator to specify a value to which a location will be set. The formats are:

\section{DIS:AAAA}

\section{SET:AAAA $=V V V V$}

where AAAA is a four digit hexadecimal memory address, and VVV is a four digit hexadecimal value to be stored in the specified memory location.

If the keyword is not in the list in Table 8.1 and bit 7 is reset, or if the keyword is not in the list or either DIS: or SET:, then a keyword error is reported to the operator by an "INVALID KEYWORD, MSG. IGNORED" message on the ASR 35. If a character used in specifying either an address or a value in a DIS: or SET: request is not a valid hexadecimal digit, an "INVALID HEX DIGIT" message is generated. If an equal sign $(\Leftrightarrow)$ does not appear between the address and the value of a SET: request, an "INVALID FORMAT" message is generated. 
After E:OMI completes those actions associated with the operator's request, control is returned to the scheduler.

\subsection{Called Subroutines}

SUBROUTINE PROGRAM FUNCTION

E:035 E\$T35 Transfers output messages to buffer before transmission to the ASR 35.

E:028 E\$T28 Translates message to BAUDOT code and stores it in buffer before transmission to the ASR 28.

E:FRT E\$FRT Reformats BCD time from the WTC to ASCII for output.

\subsection{Data Formats}

The list of valid keywords is kept in a table called MSGTB. Each entry corresponds to one keyword, and requires four computer words, interpreted as follows:

WORD 1 -- First two characters of the keyword.

WORD 2 -- Last two characters of the keyword.

WORD 3 -- Bit $\emptyset$ set for LOG: option, otherwise reset.

Bit 1 set if request is to schedule foreground task in scheduler's task table, otherwise reset.

Bit 2 set if request is for a background task, ctherwise reset.

Bits 13 - 15 hold value to which B.STT is to be set if request is for a background task, otherwise unused.

WORD 4 -- Address of the task table entry to be flagged for this request (set to schedule E:BSK for all background tasks). 


\subsection{Input Parameter Descriptions}

Address of first character of input from ASR 35.

E. IOF

E\$T35

Parameter word used in calling E:035.

E.37C

E\$T35

Count of characters in ASR 35 input buffer.

B.STT

$E \$ S K D$

Background processing status (see Table 3.2).

E.Tפ4

$E \$ S K D$ Task table entry for U\$RED.

E.Tø8

$E \$ S K D$

Task table entry for M\$STD.

E.T13

E\$SKD

Task table entry for R\$PST.

E.T15

$E \$ S K D$

Task table entry for E:SKD.

E.28F

$E \$ T 28$

Parameter word used in calling E:028.

$E: S K D$

$E \$ S K D$ Scheduler entry point.

8.5 Output Parameter Description PARAMETER SIZE DESCRIPTION E:OMI Entry point for operator message interpreting routine.

8.6 Internal Data Descriptions

PARAMETER SIZE DESCRIPTION

MSGTB

28

Table to interpret keywords (see Section 8.3)

MSGT

17

"INVALID KEYWORD,

MSG. IGNORED" message

SPACE

Space character 
8.6 Internal Data Descriptions

PARAMETER SIZE DESCRIPTION

MSG2 8 Header for log message: "123 $123456 \mathrm{~L}:$ :"

MSG2A $\quad 4 \emptyset \quad$ Buffer for operator's log message.

MSG3 11 "INVALID HEX DIGIT" message.

MSG4 9 "INVALID FORMAT" message. 


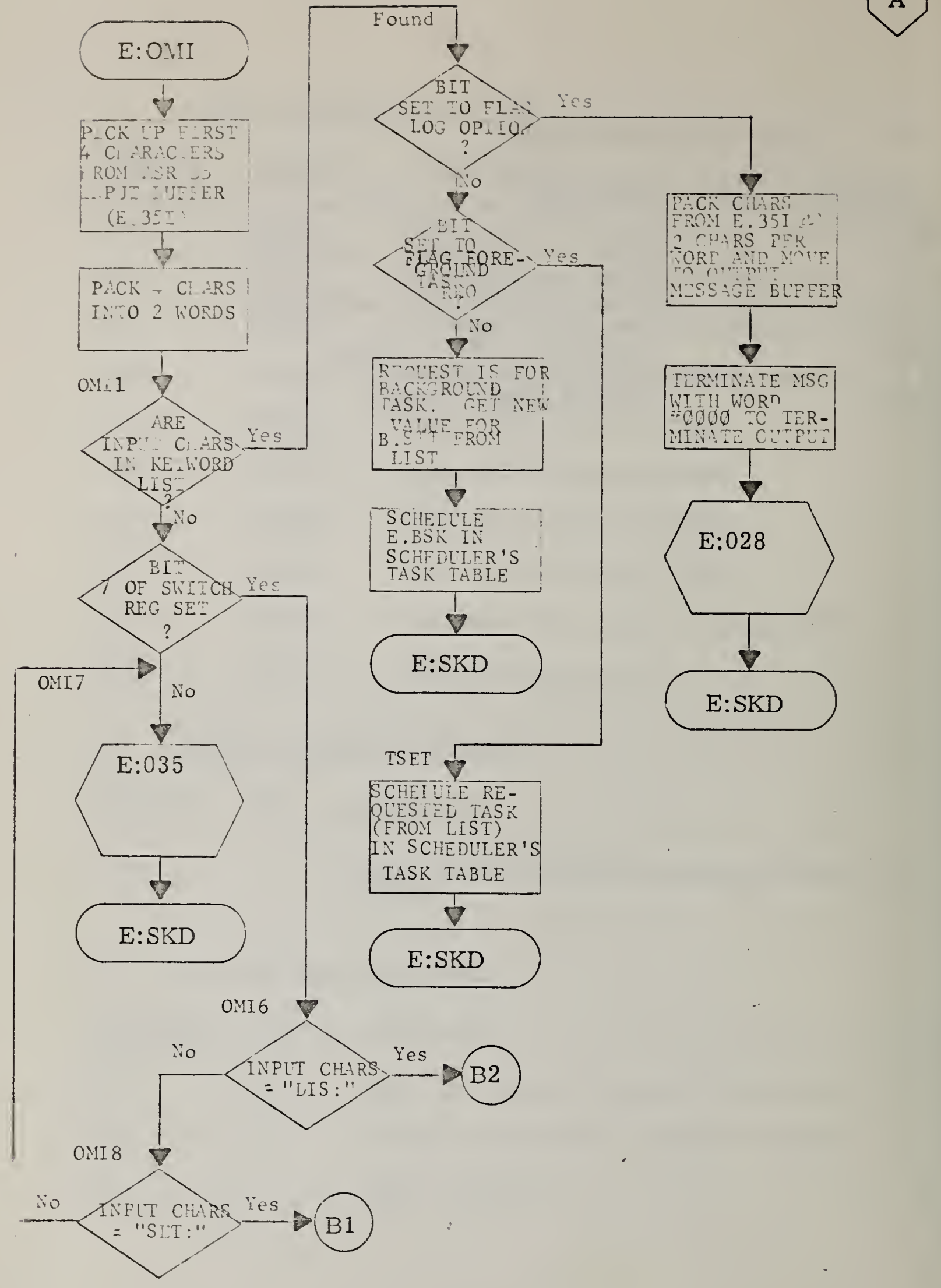




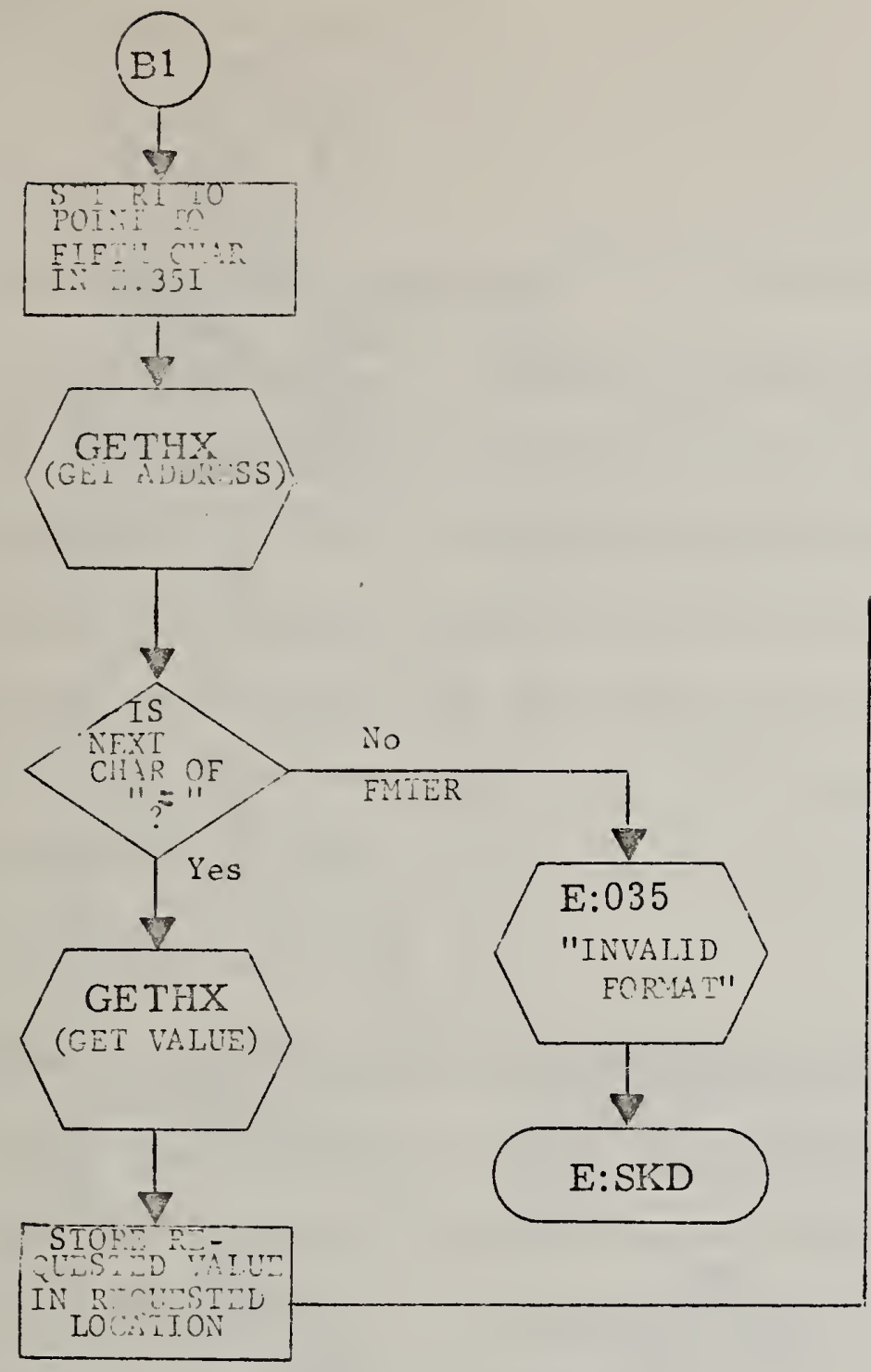

B2

IIS P:

SEI RI TO

POINT TO

IN L. 35 I

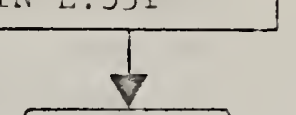

GETHX

$\langle(G E T$ ADLRECS)

(CED D.

DISIL

GET CLR TAT

QULLE OF REQUESTED

ALDR'SS FROM MEMORY
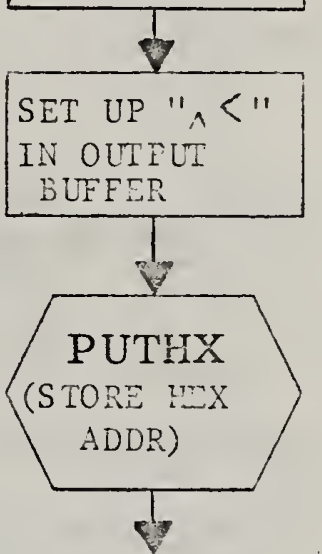
SET UP ">" IN OUTPUT BUFFLR

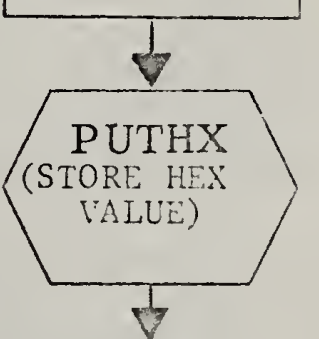

TFPINTID

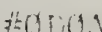

- intio

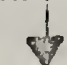

E:035

$\mathrm{E}: \mathrm{SKD}$ 
9. $E \$ T 28$

9.1 Functional Description

The ASR 28 teletype is a BAUDOT character oriented teleprinter with the capability to punch five-level paper tapes simultaneous to teleprinter operation. In NUSTAD, the ASR 28 is configured in a "receive only" mode of operation. It is used to maintain the station $\log$ and prepare paper tapes with signal descriptions for transmission over standard communications lines. E\$T28 handles all aspects of data transmissions concerning the ASR 28. It consists of three subprograms: $E: I 28, E: T 28$, and $E: 028$.

\subsubsection{E:I28 (ASR 28 Data Interrupt Service Routine)}

After the transmission of each character to the ASR 28 is completed, a data interrupt (DI) is generated which vectors control to E:I28, which schedules E:T28 in the scheduler's task table, and returns control to the interrupt program.

\subsubsection{E:T28 (ASR 28 Character Output Routine)}

After each character has been transmitted to the ASR 28, E:T28 is entered from the scheduler to determine if another character is ready for transmission, and if it is, to initiate that transfer. If the output buffer (CHRBF) is empty, then all messages in that buffer have been transmitted and the ASR 28 will go idle until another 
message is entered into CHRBF via the E:028 routine. Part III of Reportable Signal Description (RSD) messages will not be initiated until CHRBF is empty. Therefore, whenever the buffer is emptied, a flag bit in T28I0 is examined to determine if a Part III RSD is waiting. If it is, U:RP3 is flagged in the scheduler's task table before control is returned to the scheduler. If not, control is returned immediately.

\subsubsection{E:028 (ASR 28 Mess age Output Routine)}

This routine is called by a11 programs desiring to transmit data to the ASR 28 teletype. The program transiates the message from the internal storage form (ASCII characters) to the BAUDOT code, and stores the BAUDOT characters in the output buffer (CHRBF). After al1 ASCII characters have been translated (see Table 9.1 for the ASCII to BAUDOT conversion used), E:028 determines whether the teletype is a Tready in use or not. If not, it initiates output by transmitting a nu11 character to start up the I0 interface and the teletype. When this first character transmission is completed, a DI wi 11 occur, vectoring control to E:T28 through the DI service routine (E:I28) and the system scheduTer to continue further output. If the ASR 28 is a Tready outputting characters when the last message character is moved to the output buffer, the program will in effect have appended this new message to whatever output is currently underway so that the E:T28 program wi11 treat a11 output as one continuous message. E.28F is then examined to 
determine whether or not to set the reschedule bit for U:RP3 in T28I0. Control is returned to the calling program.

The station $10 \mathrm{~g}$ will contain four types of messages:
a. Routine; change of status, parameter value, etc.
b. Tables; current or historical data on system operating status.
c. Reportable Signal Descriptors.
d. Operator generated messages.

The first type is generated by $E \$ L O G$ (see Section 23) upon request of either an applications or a system program. The tables are generated by either M\$STD (Section 22) or M\$LEV (Section 21). Reportable Signal Descriptions are generated as a result of waveform analys is via U\$REP (Section 17). The final type of messages is generated by the operator using the LOG: option (see Section 8, E\$OMI).

The program transmits data to the ASR 28 by calling E:028 using the following calling sequence:
BLM, 14
E:028
DFC
(Address of first character to be transmitted)

and E.28F is set as follows:

BIT $\emptyset=\emptyset \quad$ All messages other than a Reprotable Signal Descriptor.

$=1$ If message is Part II of a RSD and the system is to schedule U:RP3 when all other output is completed. 


\begin{tabular}{|c|c|c|c|c|c|c|c|c|c|c|c|}
\hline $\begin{array}{l}\text { ASCII } \\
\text { CODE }\end{array}$ & $\begin{array}{l}\text { ASCI I } \\
\text { CHAR }\end{array}$ & $\begin{array}{l}\text { BAUDOT } \\
\text { CODE }\end{array}$ & $\begin{array}{l}\text { BAUDOT } \\
\text { CHAR }\end{array}$ & $\begin{array}{l}\text { ASCI I } \\
\text { CODE }\end{array}$ & $\begin{array}{l}\text { ASCII } \\
\text { CHAR }\end{array}$ & $\begin{array}{l}\text { BAUDOT } \\
\text { CODE }\end{array}$ & $\begin{array}{l}\text { BAUDOT } \\
\text { CHAR }\end{array}$ & $\begin{array}{l}\text { ASCI I } \\
\text { CODE }\end{array}$ & $\begin{array}{l}\text { ASCII } \\
\text { CHAR }\end{array}$ & $\begin{array}{l}\text { BAUDOT } \\
\text { CODE }\end{array}$ & $\begin{array}{l}\text { BAUDOT } \\
\text { CHAR }\end{array}$ \\
\hline$\phi \emptyset$ & NUL & $\varphi \theta$ & NUL & $2 B$ & + & $F-\not B$ & \& & 56 & V & $L-g F$ & V \\
\hline 91 & $\mathrm{SOH}$ & $8 \varnothing$ & NUL & $2 C$ & , & $F-\emptyset 6$ & , & 57 & $W$ & $L-19$ & W \\
\hline 82 & STX & $\varnothing \emptyset$ & NUL & 20 & - & $F-18$ & - & 58 & $x$ & $\mathrm{~L}-17$ & $x$ \\
\hline 93 & ETX & 90 & NUL & $2 E$ & • & $F-\varnothing 7$ & • & 59 & $Y$ & $L-15$ & $Y$ \\
\hline 94 & EOT & $9 \emptyset$ & NUL & $2 F$ & 1 & $\mathrm{~F}-17$ & I & $5 A$ & Z & {$[-1]$} & $\dot{Z}$ \\
\hline 95 & ENQ & 60 & NUL & $3 \emptyset$ & $\emptyset$ & $F-\varnothing D$ & $\emptyset$ & $5 B$ & {[} & $F-1 E$ & 1 \\
\hline 96 & ACK & 90 & NUL & 31 & 1 & $F-10$ & 1 & $5 C$ & 1 & $F-96$ & \\
\hline 97 & BEL & 90 & NUL & 32 & 2 & $F-19$ & 2 & 50 & ] & $F-99$ & $j$ \\
\hline 98 & $B S$ & 90 & NUL & 33 & 3 & $F-1 \varnothing$ & 3 & $5 E$ & $\vec{\Lambda}$ & $\phi \theta$ & NUL \\
\hline 99 & HT & $\theta \emptyset$ & NUL & 34 & 4 & $F-\not A$ & 4 & $5 F$ & 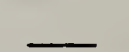 & $F-18$ & - \\
\hline$Q A$ & LF & $L-98$ & LF & 35 & 5 & $F-\emptyset 1$ & 5 & 60 & - & $F-96$ & , \\
\hline$\emptyset B$ & VT & $\phi \emptyset$ & NUL & 36 & 6 & $F-15$ & 6 & 61 & a & $L-18$ & $A$ \\
\hline OC & $\mathrm{FF}$ & $\theta \theta$ & NUL & 37 & 7 & $\mathrm{~F}-1 \mathrm{C}$ & 7 & 62 & b & $L-13$ & $\ddot{B}$ \\
\hline 90 & CR & $L-02$ & CR & 38 & 8 & $F-\varnothing C$ & 8 & 63 & $c$ & $L-Q E$ & $C$ \\
\hline$\emptyset E$ & SO & $9 \emptyset$ & NUL & 39 & 9 & $F-93$ & 9 & 64 & d & $L-12$ & D \\
\hline$\phi \bar{F}$ & SI & $\phi \phi$ & NUL & $3 A$ & : & $F-\emptyset \bar{E}$ & : & 65 & e & $L-1 \varnothing$ & $E$ \\
\hline 10 & DLE & $\phi \emptyset$ & NUL & $3 B$ & ; & $F-\emptyset \bar{F}$ & ; & 66 & $f$ & $L-16$ & $\bar{F}$ \\
\hline 11 & $\mathrm{DCl}$ & $\phi \emptyset$ & NUL & $3 C$ & $<$ & $F-1 E$ & 1 & 67 & $g$ & $L-\not B$ & G \\
\hline 12 & DC2 & $9 \emptyset$ & NUL & 30 & $=$ & $F-16$ & $i$ & 68 & h & $L-95$ & $\mathrm{H}$ \\
\hline 13 & DC3 & $\emptyset \emptyset$ & NUL & $3 E$ & $>$ & $F-\not 99$ & ) & 69 & $i$ & $L-\not C C$ & I \\
\hline 14 & DC4 & 90 & NUL & $3 F$ & ? & $F-13$ & $?$ & $6 A$ & $j$ & $L-1 A$ & $\mathrm{~J}$ \\
\hline 15 & NAK & $\emptyset \emptyset$ & NUL & $4 \varnothing$ & $\dot{a}$ & $\emptyset \emptyset$ & NUL & $6 B$ & $k$ & $L-1 E$ & $K$ \\
\hline 16 & SYN & $\varnothing \emptyset$ & NUL & 41 & A & $L-18$ & A & $6 C$ & 1 & $L-\not 99$ & $\mathrm{~L}$ \\
\hline 17 & ETB & $9 \emptyset$ & NUL & 42 & $B$ & $L-13$ & B & $6 D$ & $\mathrm{~m}$ & $L-97$ & $M$ \\
\hline 18 & CAN & $\varnothing \emptyset$ & NUL & 43 & C & $L-\not E E$ & C & $6 E$ & $n$ & $L-96$ & $N$ \\
\hline 19 & $E M$ & $9 \emptyset$ & NUL & 44 & D & $L-12$ & D & $6 F$ & 0 & $L-63$ & 0 \\
\hline $1 \mathrm{~A}$ & SVB & $9 \emptyset$ & NUL & 45 & $E$ & $L-10$ & $E$ & $7 \varnothing$ & $p$ & $L-\emptyset D$ & $P$ \\
\hline $1 \mathrm{~B}$ & ESC & $\theta \varnothing$ & NUL & 46 & $F$ & $L-16$ & $\mathrm{~F}$ & 71 & q & $L-1 D$ & $Q$ \\
\hline $1 C$ & FS & $L-1 B$ & $\mathrm{FIG}$ & 47 & G & $L-\varnothing B$ & G & 72 & $r$ & $L-\not A$ & $\mathrm{R}$ \\
\hline 70 & GS & $\phi \emptyset$ & NUL & 48 & $H$ & $L-\emptyset 95$ & $H$ & 73 & $S$ & $L-14$ & $S$ \\
\hline $1 E$ & RS & $9 \theta$ & NUL & 49 & I & $L-\not C$ & I & 74 & $t$ & $L-\not]$ & $T$ \\
\hline$\hat{T F}$ & US & $L-1 F$ & LTR & $4 A$ & $\mathrm{~J}$ & $L-1 A$ & J & 75 & $u$ & $L-1 C$ & U \\
\hline $20 \mathrm{~s}$ & pace & $L-04$ & space & $4 B$ & K & $L-1 E$ & $k$ & 76 & v & $L-\emptyset F$ & V \\
\hline 21 & $!$ & $F-16$ & & $4 C$ & L & L- 99 & L & 77 & $w$ & L-19 & W \\
\hline 22 & $"$ & $\mathrm{~F}-11$ & 11 & 40 & $M$ & $L-97$ & $M$ & 78 & $x$ & $\mathrm{~L}-17$ & $x$ \\
\hline 23 & $\#$ & $F-\not 5$ & NP & $4 E$ & $N$ & $L-96$ & $N$ & 79 & $y$ & $L-15$ & $\ddot{Y}$ \\
\hline 24 & $\$$ & $\mathrm{~F}-12$ & $\$$ & $4 F$ & 0 & $L-\square 3$ & 0 & $7 A$ & $z$ & L-11 & Z \\
\hline 25 & $\%$ & $\mathrm{~F}-17$ & 1 & 50 & P & $L-\emptyset D$ & P & $7 B$ & \{ & $\bar{F}-1 \mathrm{E}$ & ( \\
\hline 26 & $\&$ & $F-\not B$ & 2 & 51 & $Q$ & L-1D & $Q$ & $7 C$ & ! & $F-\emptyset 6$ & , \\
\hline 27 & 1 & $F-1 A$ & 1 & 52 & $R$ & $L-\varnothing A$ & $\mathrm{R}$ & 70 & \} & $F-99$ & j \\
\hline 28 & ( & $F-1 E$ & ( & 53 & $S$ & $L-14$ & $S$ & $7 E$ & 2 & $\phi \emptyset$ & NUL \\
\hline 29 & j & $F-\not 99$ & j & 54 & $T$ & $L-\varnothing 1$ & $T$ & $7 \bar{F}$ & DEL & $F-14$ & BEL \\
\hline $2 A$ & * & $F-1 A$ & $i$ & 55 & U & $L-1 C$ & U & & & & \\
\hline
\end{tabular}

NOTE: BAUDOT code transmits in two modes. $L$ is used to denote characters in the LTR mode, and F to denote characters in the FIG mode. Both ASCII and BAUDOT codes are shown in hexadecimal. 
A11 characters in consecutive memory locations beginning at the location specified will be translated and stored for output until a null character $\left(\varphi \emptyset_{16}\right)$ is reached. This will be considered as a terminator for the output message.

\subsection{Data Formats}

\subsubsection{Conversion Table Entries}

The table used to equate ASCII characters with the BAUDOT equivalents as given in Table 9.1 is called BAUDT. Each ASCII character corresponds to one character (byte) in the table. The Most Significant Bit (MSB) of the byte is set if the character in that byte is in the FIG mode. The actual bits of the BAUDOT character are stored in the five Least Significant Bits of that byte, in reverse order. For example, the letter "A" is normally represented in BAUDOT as an $18{ }_{16}$. In BAUDT it is stored as 9316 .

\subsubsection{FLAG}

During the translation process, mode change characters ( $1 \mathrm{~B}_{16}$ to FIG and $1 F_{16}$ to $L T R$ ) are transmitted only when the next character is not in the same mode as the iast character translated. Therefore, bit 8 of FLAG is used to determine the mode of the last character moved to CHRBF. If bit 8 is set, the last character considered was in the FIG mode, and a mode change character will not be needed until the next character is in the LTR mode. 


\section{$9.2 .3 \quad \mathrm{~T} 2810$}

T28I0 maintains all necessary status information concerning the ASR 28. It is interpreted as follows:

BIT $\emptyset=\emptyset$ ASR 28 is idle, no output in progress.

$=1$ Output is underway to the ASR 28.

BIT $1=\emptyset$ No report message (Part III of RSD) waiting.

$=1$ Need to schedule U:RP3 first time CHRBF becomes empty.

BIT 2 = 0 Normal output mode.

$=1$ Next DI will be to output first character from CHRBF.

\subsection{Input Parameter Descriptions}

PARAMETER PROGRAM DESCRIPTION

E:SKD E\$SKD Scheduler entry point.

E.T05 E\$SKD Task table entry for E:T28.

E.T97 E\$SKD Task table entry for U:RP3.

9.4 Output Parameter Descriptions

PARAMETER SIZE DESCRIPTION

E.28F 1 Parameter used in calling E:028 (see Section 9.1.3).

E.280 1 External name given to T2810 (see Section 9.2.3).

E:I28 Entry point to ASR 28 DI service routine.

E:T28 Entry point to ASR 28 character output routine.

E:028 Entry point to BAUDOT character translator and buffering routine. 
9.5 Internal Data Descriptions

PARAMETER SIZE DESCRIPTION

BAUDT 64 Table of ASCII to BAUDOT character equivalents (see Section 9.2.1).

PNTIN 1 Pointer to next location in CHRBF into which a character may be stored by E:028.

PNTOT 1 Pointer to next character to be taken from CHRBF by E:T28 for transmission to the ASR 28.

CHRBF 2948 Output buffer for characters for transmission to the ASR 28.

T28I0 1 ASR 28 status word (see Section 9.2.3).

CONTR 3 The three control words necessary to initiate a character transfer to the ASR 28.

SAVRS $\quad 7$ Register holding buffer.

FLAG $\quad 1$ Bit $8=\emptyset$ if last character moved to CHRBF by E:028 was in the LTR mode, = 1 if it was FIG mode (see Section 9.2.2). 

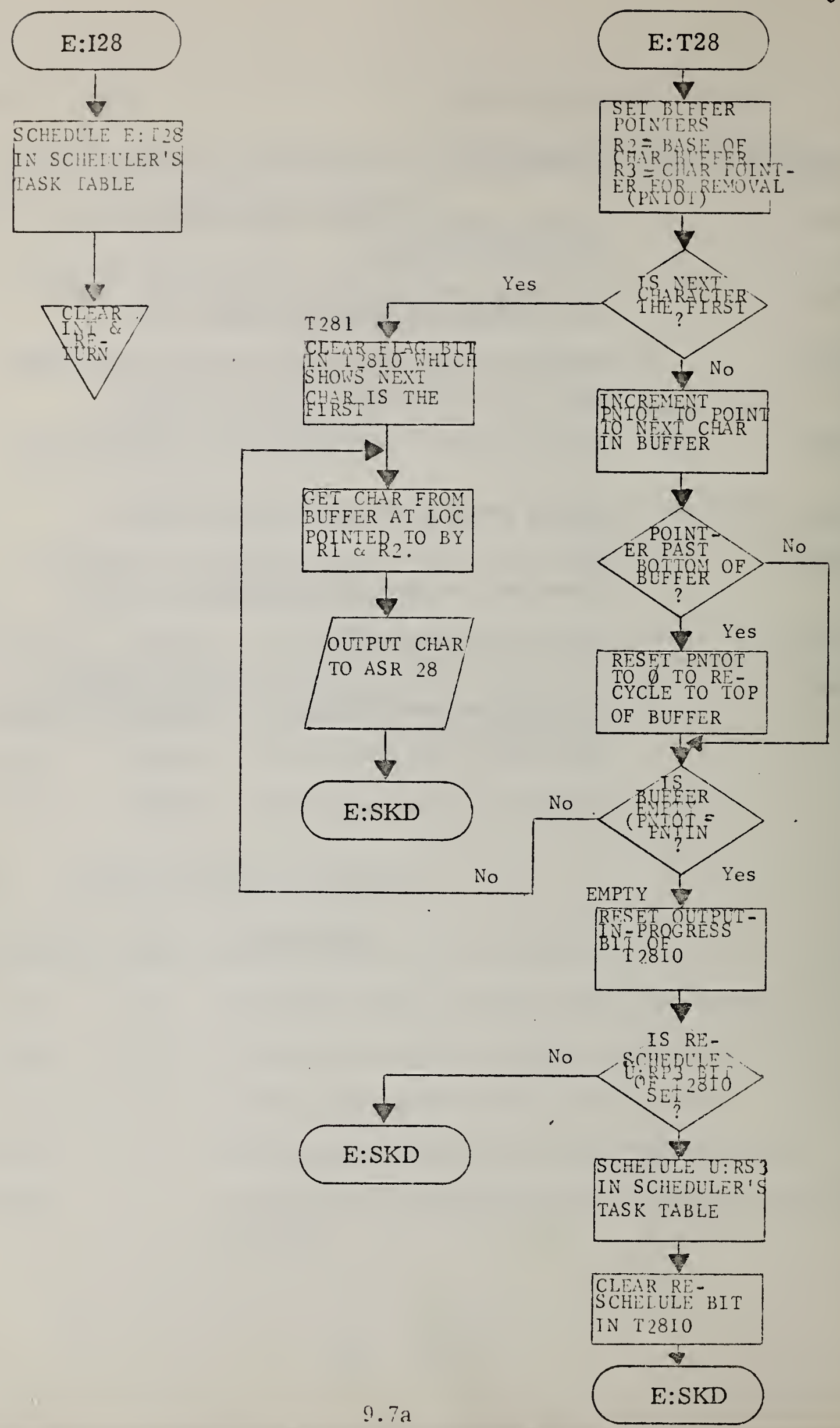


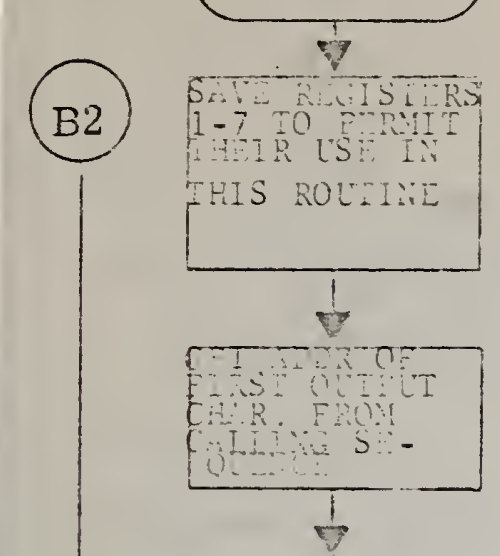

CORRECI KETLRY

ADIR TO E.T.?

E-R IOUT:

IOS

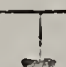

SET IXYT BYTE

POIN IFR ROR

BITE SIOKACE

POINIER
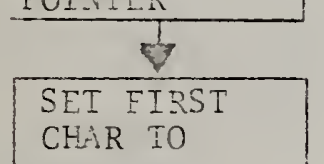

$\because 1 F$ (IITR)

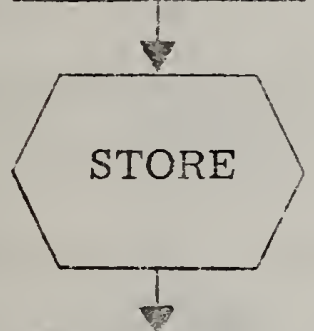

RESEI IIOD

IIR $\because O D E$

गTारण है

[CFT IFY NSCI

CHIR FROM LOC

DTRTYED FROM

PARit.

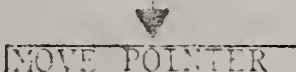

10 NEXI ASCII

CHLARACIER
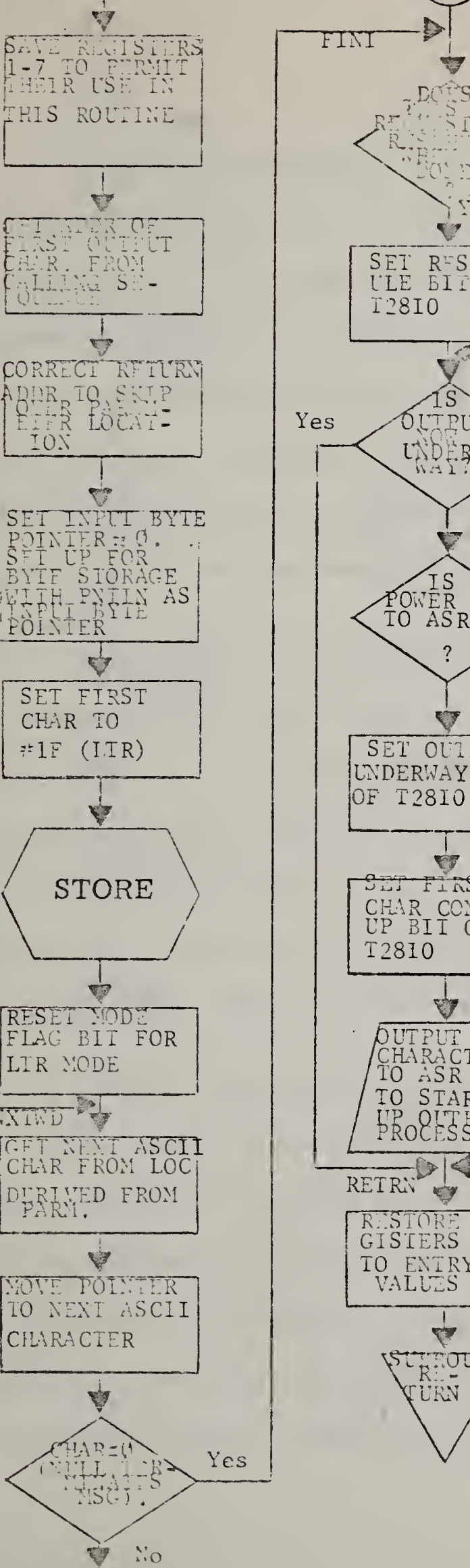


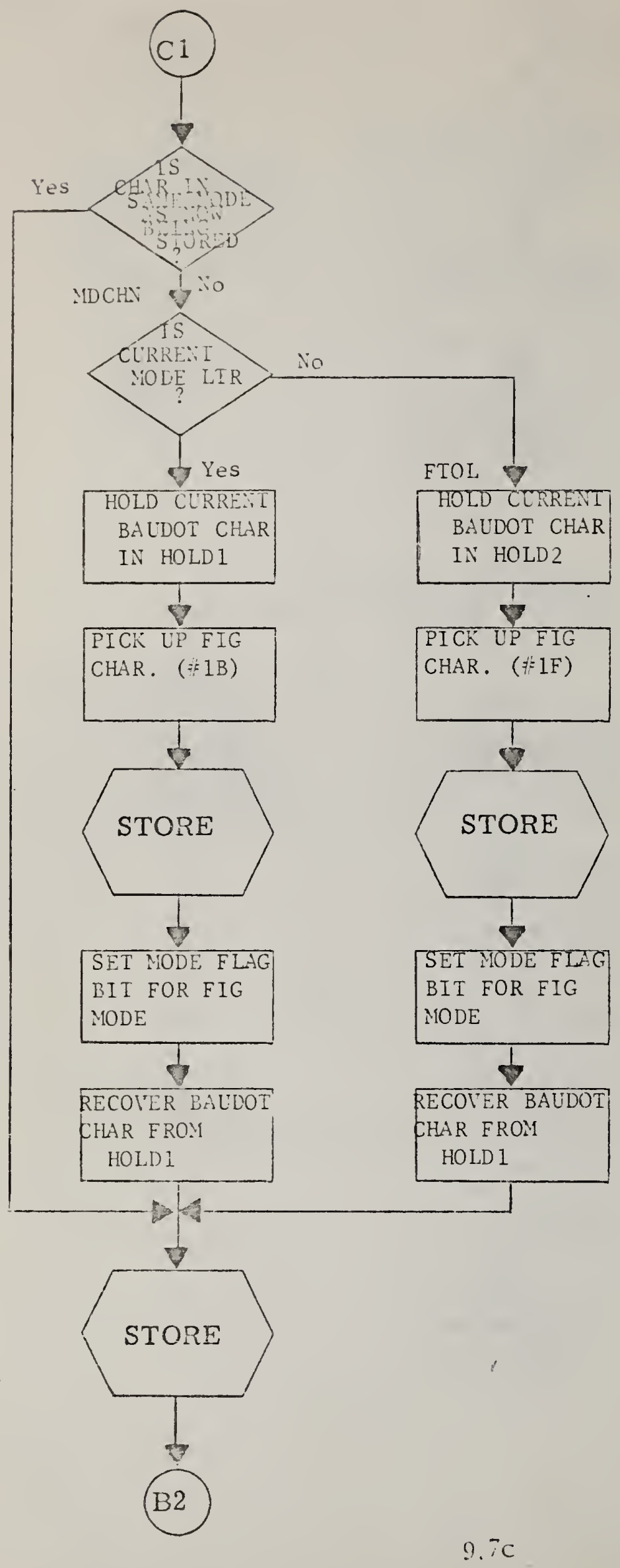

\section{STORE}

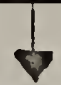

STORE BAUDOT

CHAR. IN OLT-

PLT BLFFER AI

BQC POINTED IO

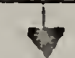

TNCRENENT PNTTN

TOXOLATA TOC Torifit
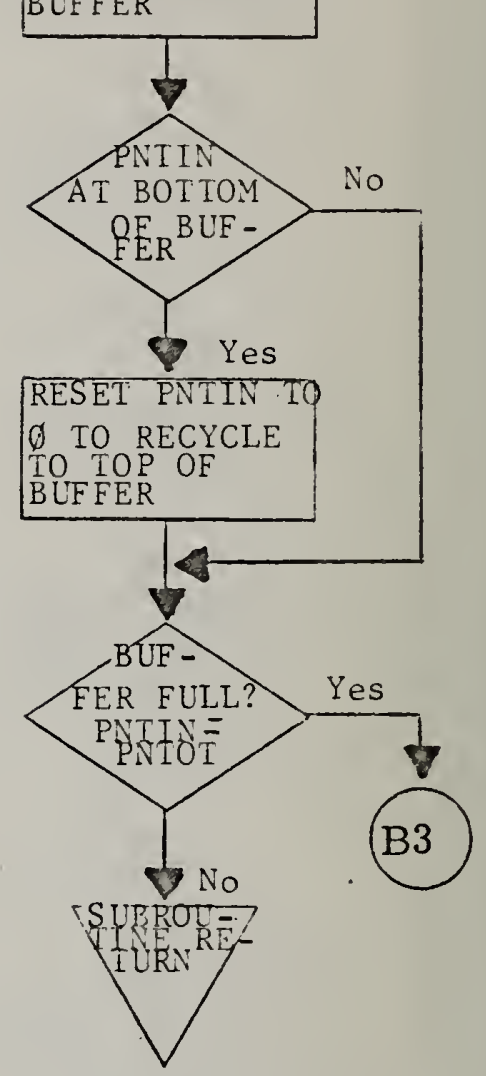
10. $E \$ T 35$

10.1 Functional Description

The ASR 35 teletype is an ASCII character oriented teleprinter with the capability to punch eight-level paper tapes simultaneous to teleprinter operation. In NUSTAD, the ASR 35 is used to provide the operator with a means to communicate with the operating system, and provide the control necessary to the execution of background tasks. E\$T35 handles all aspects of data transmissions concerning the ASR 35 . It consists of three subprograms: $E: 135, E: T 35$, and $E: 035$.

\subsubsection{E:I35 (ASR 35 Data Interrupt Service Routine)}

On input, after the hardware character buffer contains a character as the result of the operator depressing a key on the ASR 35 keyboard, or on output as the transfer of a character from the hardware character buffer to the teleprinter is completed, a data interrupt (DI) is generated which vectors control to $E: I 35$, which schedules E:T35 in the scheduler's task table, and returns control to the interrupted program.

\subsubsection{E:T35 (ASR 35 Character Input/Output Routine}

Since the ASR 35 is configured for both input and output, and the same interrupts are used for both functions, NUSTAD must always know what the ASR 35 is currently being used for. This is done by means of a status word called T35IO. Whenever E:T35 is entered from the system scheduler after a DI, its response depends upon what role the ASR 35 is currently playing. Basically, the roles may be considered three: 
a. Awaiting the operator to request an action through the use of a keyword input (See Section 8.1 for a discussion of keywords). b. Awaiting the operator to respond to a background program request for information.

c. Busy outputting characters to the ASR 35 as specified by a NUSTAD program.

We may summarize these states as being either input ( $a$ and $b$ ) or output (c); and if awaiting input, then either the REQUEST mode (a) or RESPONSE mode (b).

If the ASR 35 is currently being used for output, each entry to E:T35 signals the end of one character's transmission; thus the teleprinter is ready to receive another. If another character is available in the output buffer, it is transmitted. Otherwise, the buffer is considered empty and output is terminated. When the buffer is emptied, status bits are checked in T35I0 to determine if the output is from a background task and whether that task is ready to resume processing. If it is, E:BSK is rescheduled. In either case, a lack of output puts the ASR 35 into an input state.

Normally, the ASR 35 is in the REQUEST mode. The operator calls NUSTAD's attention by typing the slash character (/). E:T35 detects this as the only valid initial character in the REQUEST mode, and crosses it to form the letter $x$, informing the operator that NUSTAD 
is ready to accept a keyword input. When the operator terminates his input by typing a carriage return character, E\$OMI is scheduled in the scheduler's task table and control is returned to the scheduler.

If an output request from a background program sets a flag bit to identify a request for an operator response, then the ASR 35 automatically goes into the RESPONSE mode when the output buffer becomes empty. When the operator terminates his response with a CR, NUSTAD will reschedule the background scheduler (E:BSK) in the task table to permit the background task to resume processing.

\subsubsection{E:035 (ASR 35 Message Output Routine)}

Any foreground program (or B:IOR for background programs) which desires to output to the ASR 35 does so by calling E:035. This rcutine transfers the message into an output buffer. When the transfer is complete, T35I0 is examined to determine if input is actually going on. If it is, T35I0 is flagged to indicate that output is pending, to be started as soon as the current input is terminated. If there is no input underway, E:035 checks to see if output is underway. If it is, the current message wi 11 be considered as a continuation of the current output stream and nothing further need be done. If neither input nor output is underway, the output is initiated by issuing a terminate command to the ASR 35. When this is received, a service interrupt (SI) is received, vectoring control to $E: 35 S$ where the first of two nul1 
characters is transmitted. E:035 returns control to the calling program after checking the desired options as requested in E.IOF and setting T35I0 accordingly.

The calling sequence to initiate an output to the ASR 35 is: $\mathrm{BLM}, 14 \quad \mathrm{E}: 035$

DFC (Address of first character to be transmitted)

and E.IOF set as follows:

BIT $\emptyset$ Do not reschedule E:BSK immediately after the output is completed. This option is used by foreground tasks with output to the ASR 35 or by background tasks in conjunction with BIT $1=1$ to permit the background to request information from the operator, then not reenter the background program unitil the operator's response has been read.

$=1$ Reschedule E:BSK as soon as the output buffer is empty.

BIT $1=\emptyset$ No response to the program's output is expected from the operator.

$=1$ The message now in the output buffer is a request by a background program for information. Do not reschedule E:BSK until the input is complete. The ASR 35 will be put into the RESPONSE mode when the the output buffer is emptied.

A11 characters in consecutive memory locations beginning at the Tocation specified will be moved to the output buffer (two characters per word) until a null word $\left(00 \emptyset_{16}\right)$ is encountered. This will be considered as a terminator for the output message.

While entering his input data stream, the operator may use two options designed to permit text editing: 
a. The most previous character entered may be deleted from the input buffer by typing a RUB OUT character. This feature may be repeated to delete several letters to the point where the initial system call request is deleted, at which point E:T35 wiil disregard the entire message and reset flags to begin receiving input anen:

b. The entire message may be disregarded by typing a CAN, entered by simultaneously depressing the CONTR and $X$ keys. Input may then begin anew for that message.

E:T35 expects the first character entered while in the REQUEST mode to be the slash (/). If it is not, an "INVALID SYSTEM CALL" message is generated and the message is disregarded.

10.2 Data Formats

10.2.1 T35I0

The current status of I0 operations with the ASR 35 is maintained in T3510. The bits of this status word are interpreted as follows: BIT $\varnothing=\varnothing \quad$ Input is expected. The ASR 35 is set to respond to input characters entered from the keyboard, but has not yet received the first character of an input message.

$=1$ Output is currently in progress to the ASR 35. Further output requests are not needed as additional messages will be appended as a continuation of the current data stream. Input is inhibited until output is complete. 
BIT $1=\emptyset \quad$ Data input is awaiting the first character of an input message.

$=1$ Input message is underway; i.e., past the first character.

BIT $2=\emptyset$ Input is in the REQUEST mode. Input message will be processed by $E \$ O M I$.

$=1$ Input is in the RESPONSE mode. Termination of input message will cause E:BSK to be rescheduled.

BIT $3=\emptyset \quad$ No output messages have been entered into the output buffer while current input has been underway.

$=1$ At least one program has requested a message to be output to the teleprinter while input was underway. When the input is completed, initiate the sequence to begin the character output transfer.

BIT $4=\emptyset \quad$ The ASR 35 is not currently busy with output.

$=1$ The ASR 35 currently has output underway.

BIT $5=\emptyset \quad$ Do not reschedule E:BSK after the output is completed. If the output is generated by a background program, an operator response is expected before background processing may be resumed.

$=1$ When the output buffer becomes empty, reschedule E:BSK to permit the background task to resume processing.

BIT $6=\emptyset \quad$ Normal output underway.

$=-1$ The current DI follows the first null character transfer, transmit another null as the next output character.

BIT $7=\emptyset \quad$ Normal output underway.

$=1$ Second nutl completed, begin with first character from output buffer. 
10.3 Input Parameter Descriptions

$\begin{array}{lll}\text { PARAMETER } & \text { PROGRAM } & \text { DESCRIPTION }\end{array}$
E.Tø6
$E \$ S K D$
Task table entry for E\$OMI
E.T12
$E \$ S K D$
Task table entry for E:T35
E.T15 E\$SKD
Task table entry for background system (E:BSK)
$E: S K D$
$E \$ S K D$
System scheduler entry point

10.4

Output Parameter Descriptions

PARAMETER

SIZE

DESCRIPTION

E. 35I

80

Input character buffer, one character per word. Input character stream terminated by word $=\varnothing \emptyset \phi \emptyset_{16}$

E.37C $\quad 1$

Counter of input characters in input buffer. Used as a pointer to indicate where to store next character during actual input.
E. IOF
1
Parameter word used with call to E:035
E. 350
1
External reference to T35I0 (See Section 1פ.2.1)

10.5

Internal Data Descriptions

PARAMETER SIZE DESCRIPTION

T35I0

1

FOUL 1

13

G00DI

3

CRLF

2

OUTBF

256

PNTIN

1

PNTOT

SAVRS
ASR 35 processing status (See Section 10.2.1)

"INVALID SYSTEM CALL" message

Backslash response to valid system call

Carriage return and Line feed

Output character buffer, pack two characters per word

Pointer to next location in OUTBF into which a character may be stored by E:035

Pointer to next character to be taken from OUTBF by $E: T 35$ for transmission to the ASR 35

Register holding buffer

Register holding buffer 

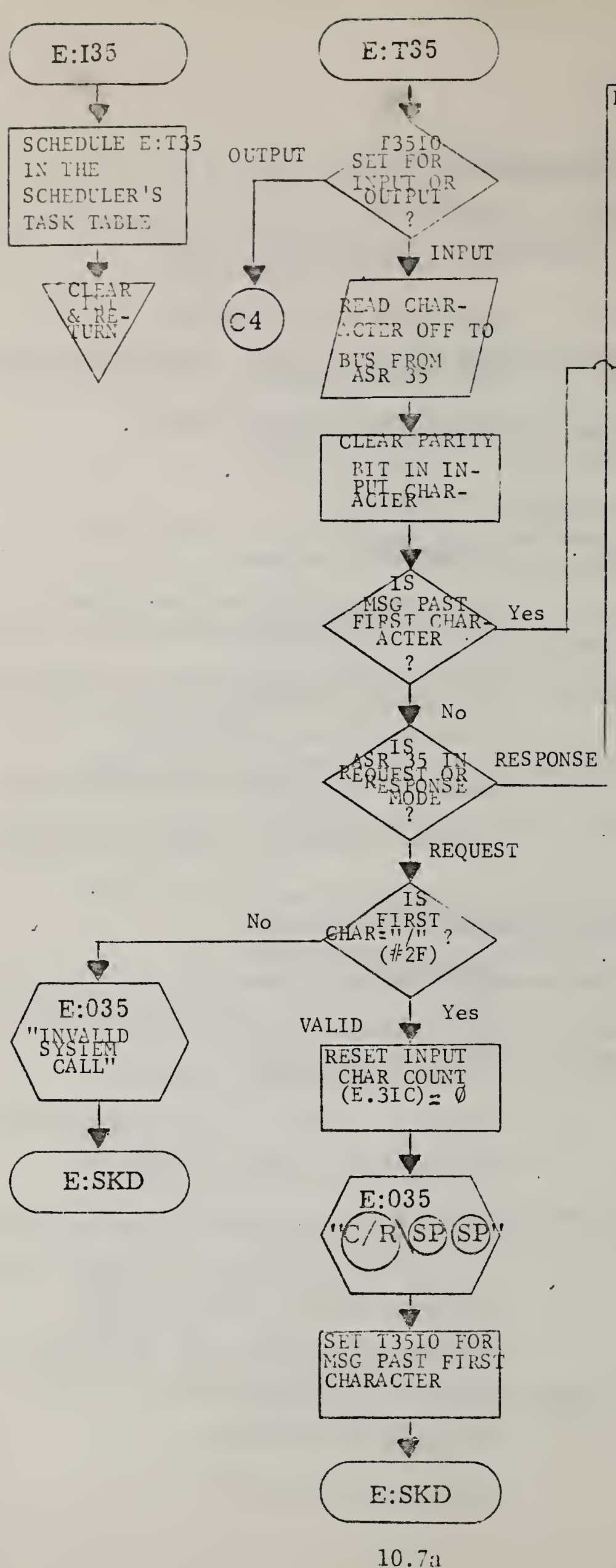

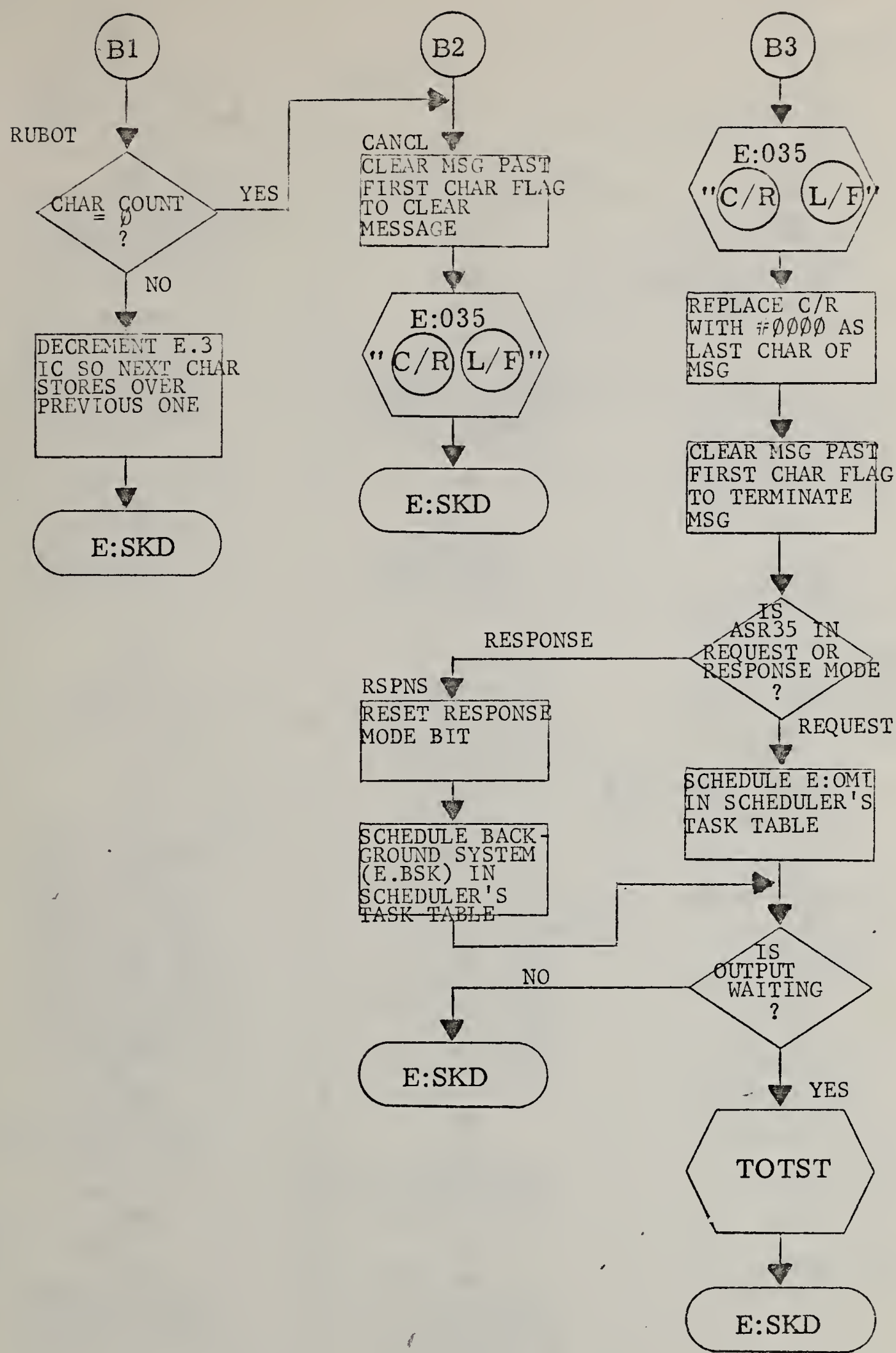


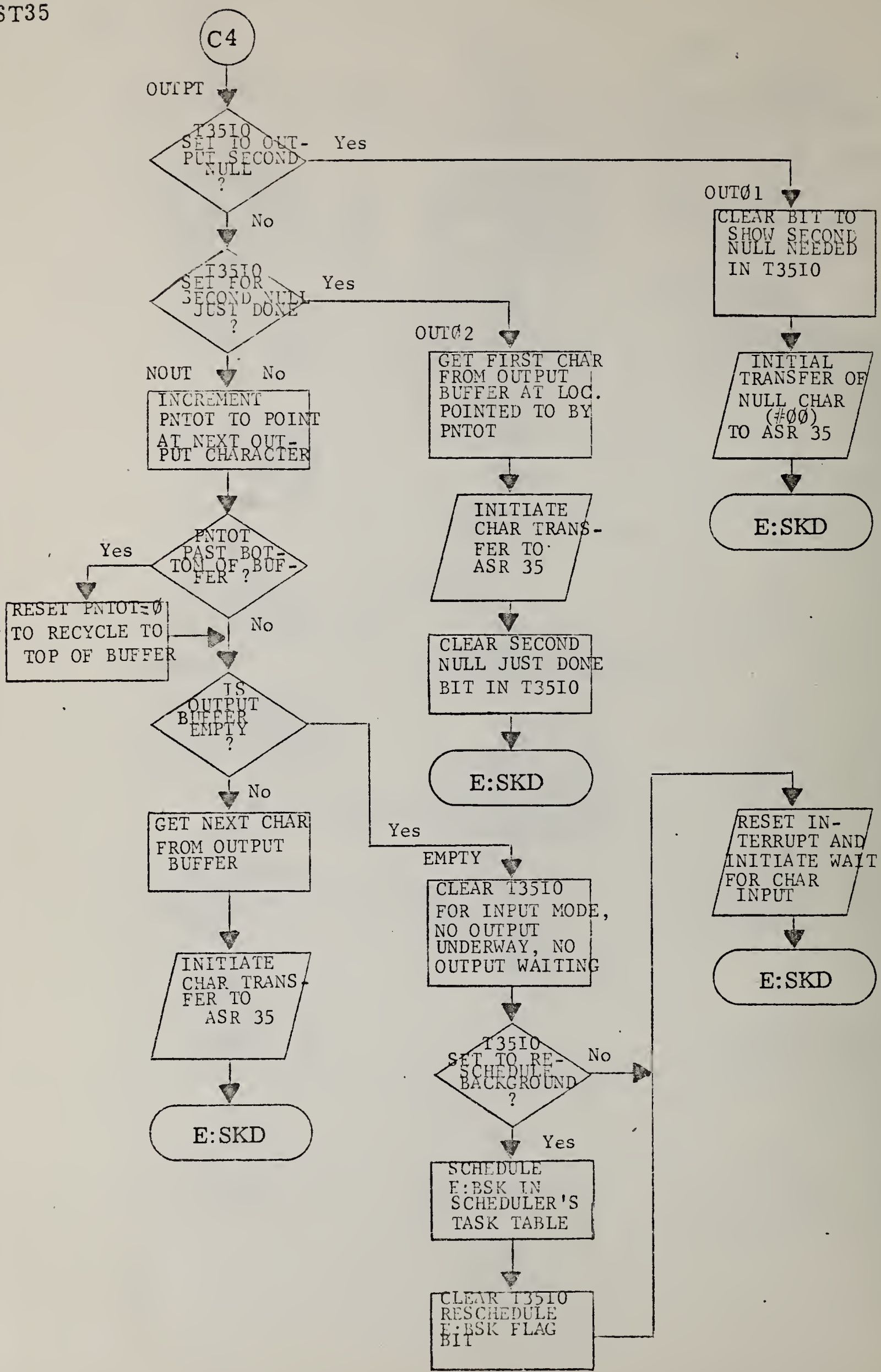

$10.7 \mathrm{C}$ 

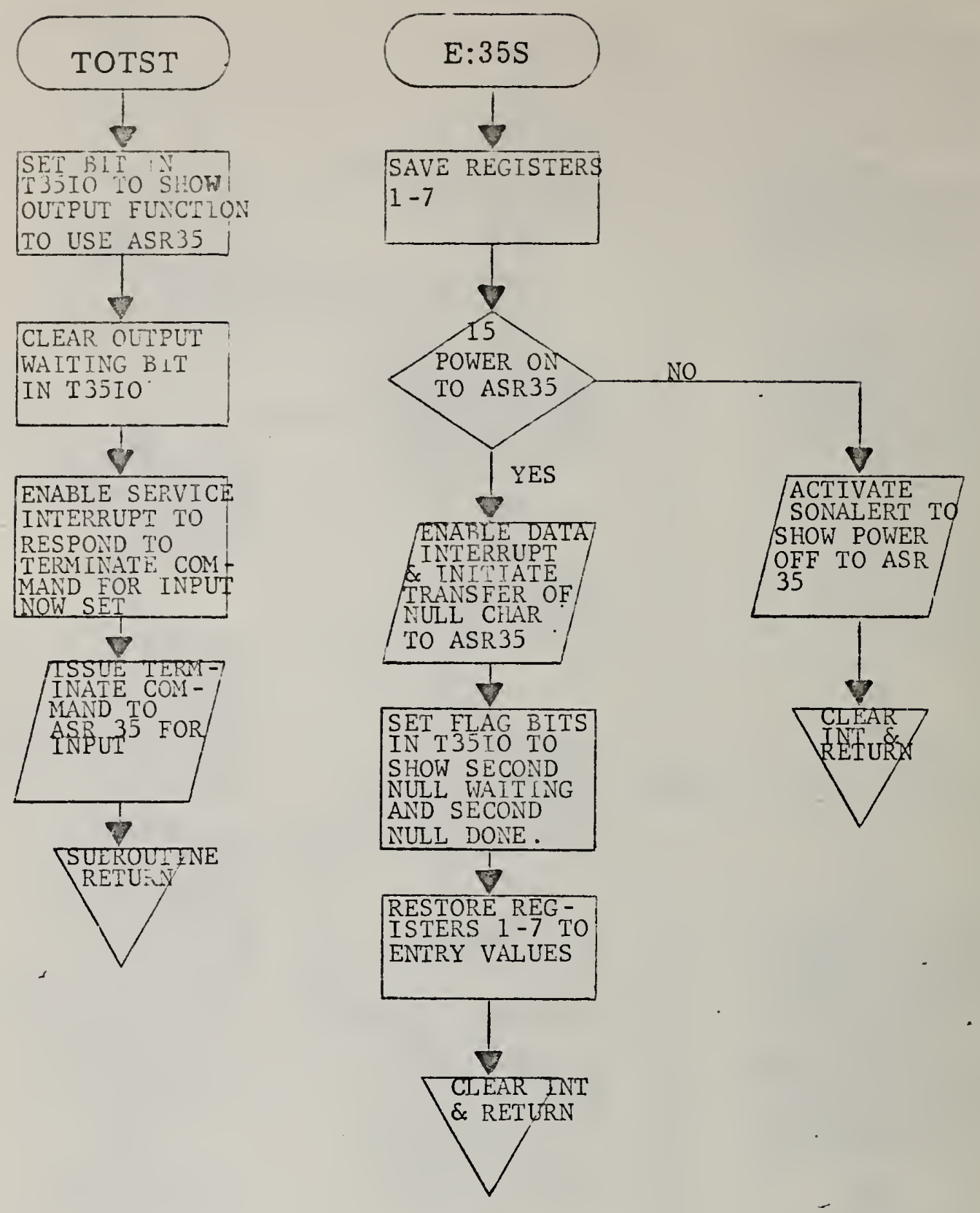
11. E\$MAG

11.1 Functional Description

The WANG 45 ips nonvacuum tape drive is used to process 7 -track IBM compatible magnetic tapes. In NUSTAD, this device is dedicated to the input of DRFUD data from tapes previously recorded on the DRFUD units. E\$MAG consists of three routines, E:MTI, M:MAG, and M:MGS.

\subsubsection{E:MTI (Mag Tape SI Service Routine)}

Whenever a mag tape data transfer (via DMP) is completed, a service interrrupt (SI) vectors control to the interrupt service routine, E:MTI. This routine flags E:MAG in the scheduler's task table and returns control to the interrupted program.

\subsubsection{E:MAG (Mag Tape Error Checking Routine)}

$E: M A G$ is entered from the scheduler to examine a completed data transfer from the mag tape unit. It reads the hardware status for that transfer and checks for the following errors:

a. If switch register bit 2 is reset, E:MAG wi11 record parity errors wi th a "MAG TAPE PARITY ERROR, CALLING TASK ABORTED" message. If switch register bit 2 is set, parity errors will be ignored.

b. Tape defects will be brought to the operator's attention with a "TAPE DEFECT DETECTED, CALLING TASK ABORTED" message.

c. If the end of tape marker is discovered, an "END OF TAPE DETECTED, CALLING TASK ABORTED" message is generated.

d. If the tape unit became inoperative during the transfer, a "TAPE UNIT INOPERATIVE, CALLING TASK ABORTED" message is generated. 
If any of these errors are reported to the operator on the ASR 35, the background task is aborted (B.STT is set to $\emptyset$ ), and the data transfer is ignored. If, however, no errors are discovered, E:BSK is rescheduled to permit the current background task to resume processing and control is returned to the scheduter.

\subsubsection{E:MGS (Mag Tape Input Initiate Routine)}

Currently, the only program which requests a mag tape data transfer is $B: I O R$, which does so for the background programs processing DRFUD data tapes. The calling sequence for a data read from mag tape is:

BLM, 13 E:MGS

DFC (Memory address into which first word to be stored)

DFC (Count of words to be transferred)

The two parameters are stored into the DMP transfer address and transfer count (after negating the TC).

The status of the equipment is read and if it is either off-line, not ready, or without power, a "MAG TAPE NOT READY, CALLING TASK ABORTED" message is generated and the background task underway is aborted. If all equipment is ready for the transfer, it is initiated, and control is returned to the calling routine. 
$11.2 \quad$ Called Subroutines

SUBROUTINE PROGRAM

FUNCTION

E:035 E\$T35 Transfers output message to buffer before transmission to the ASR 35.

11.3 Input Parameter Description

PARAMETER PROGRAM DESCRIPTION

$E: S K D$

E\$SKD

System scheduler's entry point.

E.T11

$E \$ S K D$

Task table entry for E:MAG.

E.T15

$E \$ S K D$

Task table entry for background scheduler (E:BSK)

E. IOF

E\$T35

Parameter used in calling E:035.

B.STT

E\$T35

Background processing status (See Table 3.2)

F.MTP

F\$FMT

Mag tape buffer word pointer.

11.4 Output Parameter Descriptions

PARAMETER SIZE DESCRIPTION

E:TMI

Entry point for mag tape SI service routine.

$E: M A G$

Entry point for mag tape transfer completed routine.

E:MGS

Entry point for input request routine for mag tape.

$11.5 \quad$ Internal Data Descriptions \begin{tabular}{lll} 
PARAMETER SIZE DESCRIPTION \\
\hline
\end{tabular}

MD 1

24

Mø2

14

Mø3

$M 94$

ind 5

14

Mø்6

TEMP
"MAG TAPE NOT READY, CALLING TASK ABORTED." message.

"MAG TAPE PARITY ERROR," message

"TAPE DEFECT DETECTED," message

"END OF TAPE DETECTED," message

"TAPE UNIT INOPERATIVE," message

"CALLING TASK ABORTEU." message

Register holding buffer. 


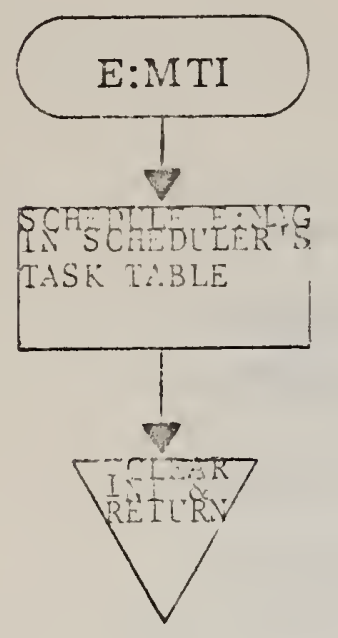

READ HARDKARE

GENERAIED

SIATLS WORE

FOR LAST DAIA

TRANSFER
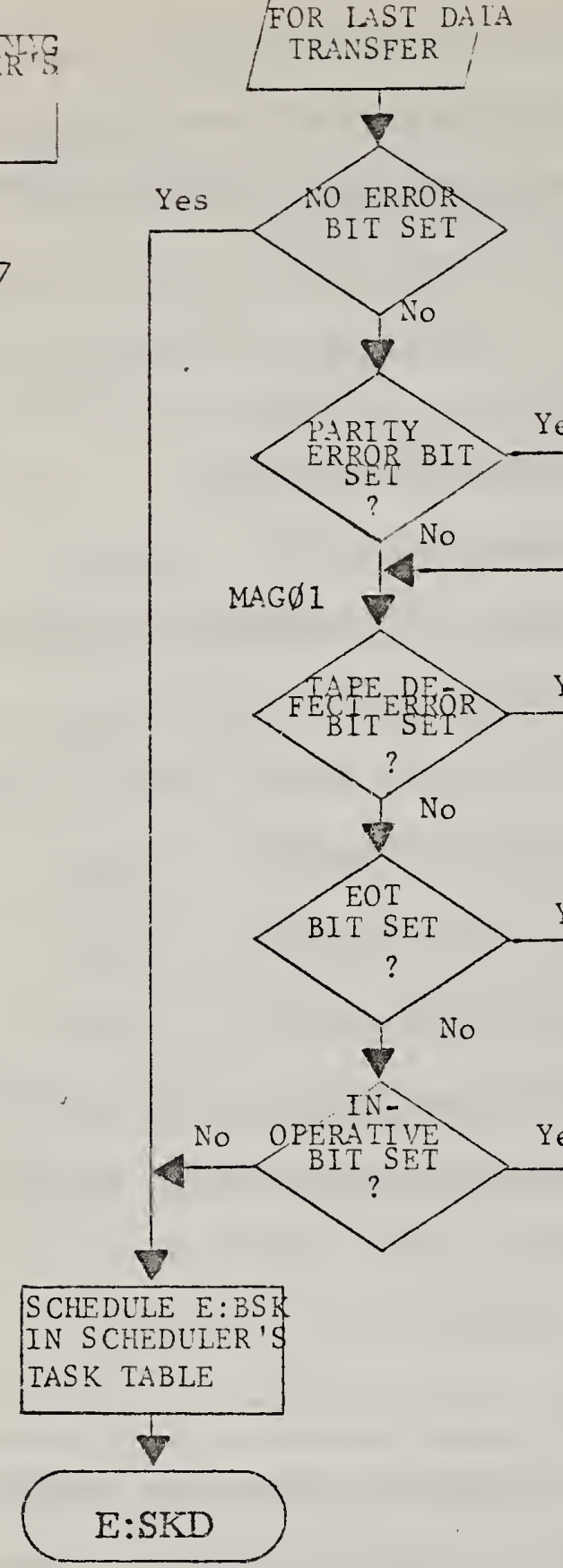

BIT SET
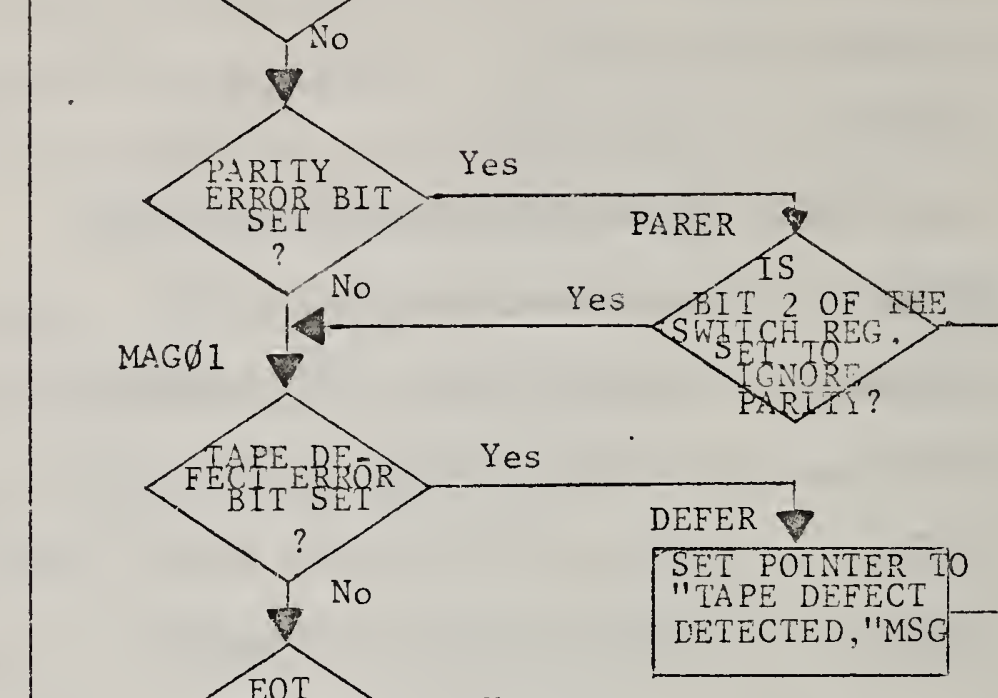

BIT SET
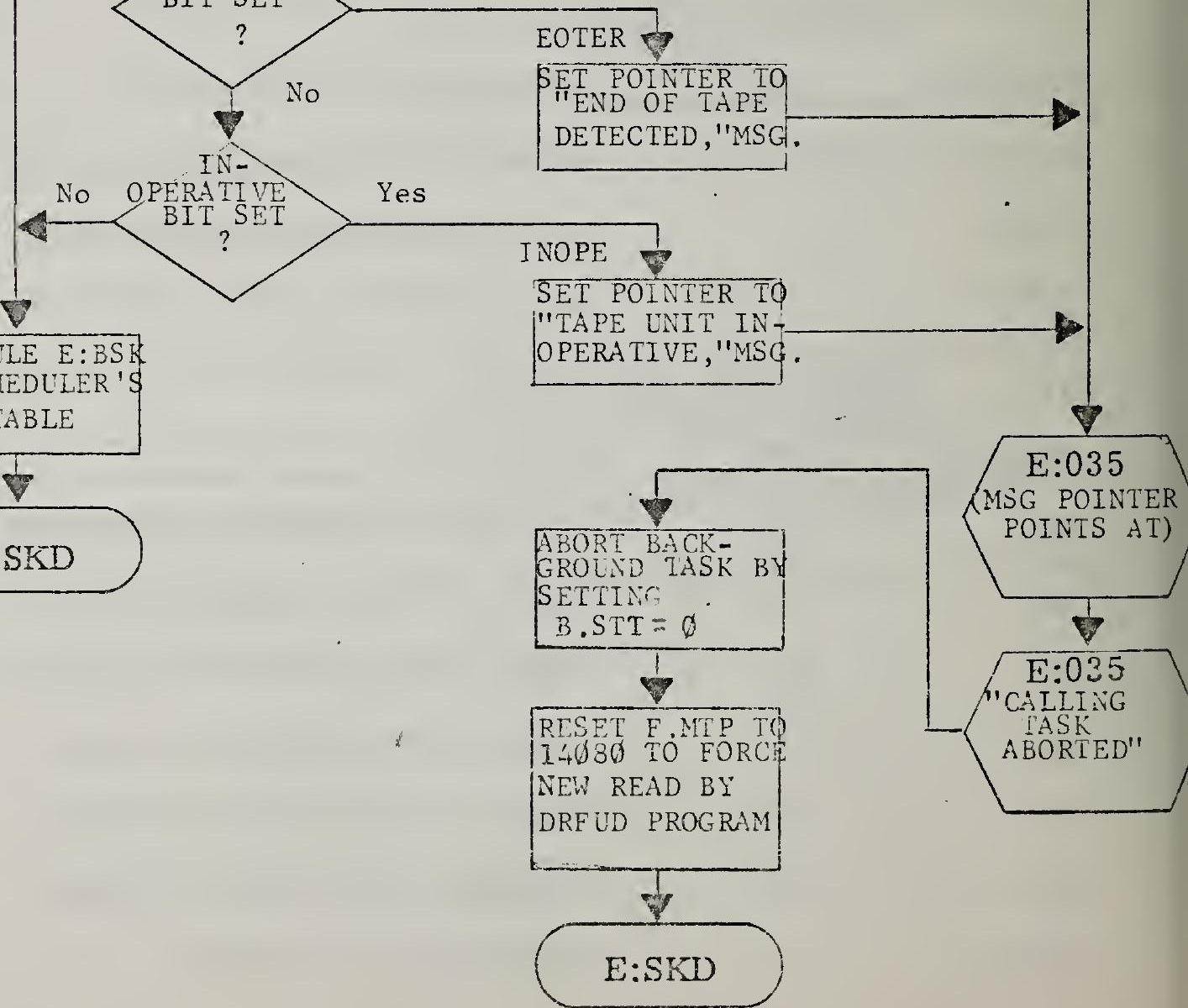


\section{E:MIGS}

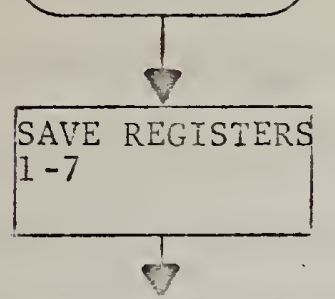

CEI FIRST PARM

WORD FROY CAL -

SAORE SUE IOE \&

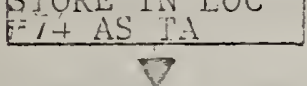

CEFT SECOND PAFM

WORD FROY CAL

UNC SEQUENCE

IN LOC \#64 AS
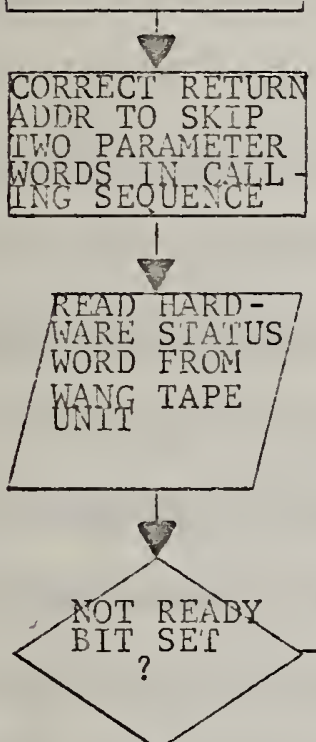

DIT READY

IT SE'
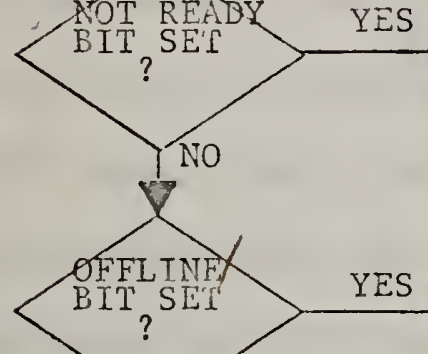

NO

OFFLINEA

$\mathrm{BIT}$ ?
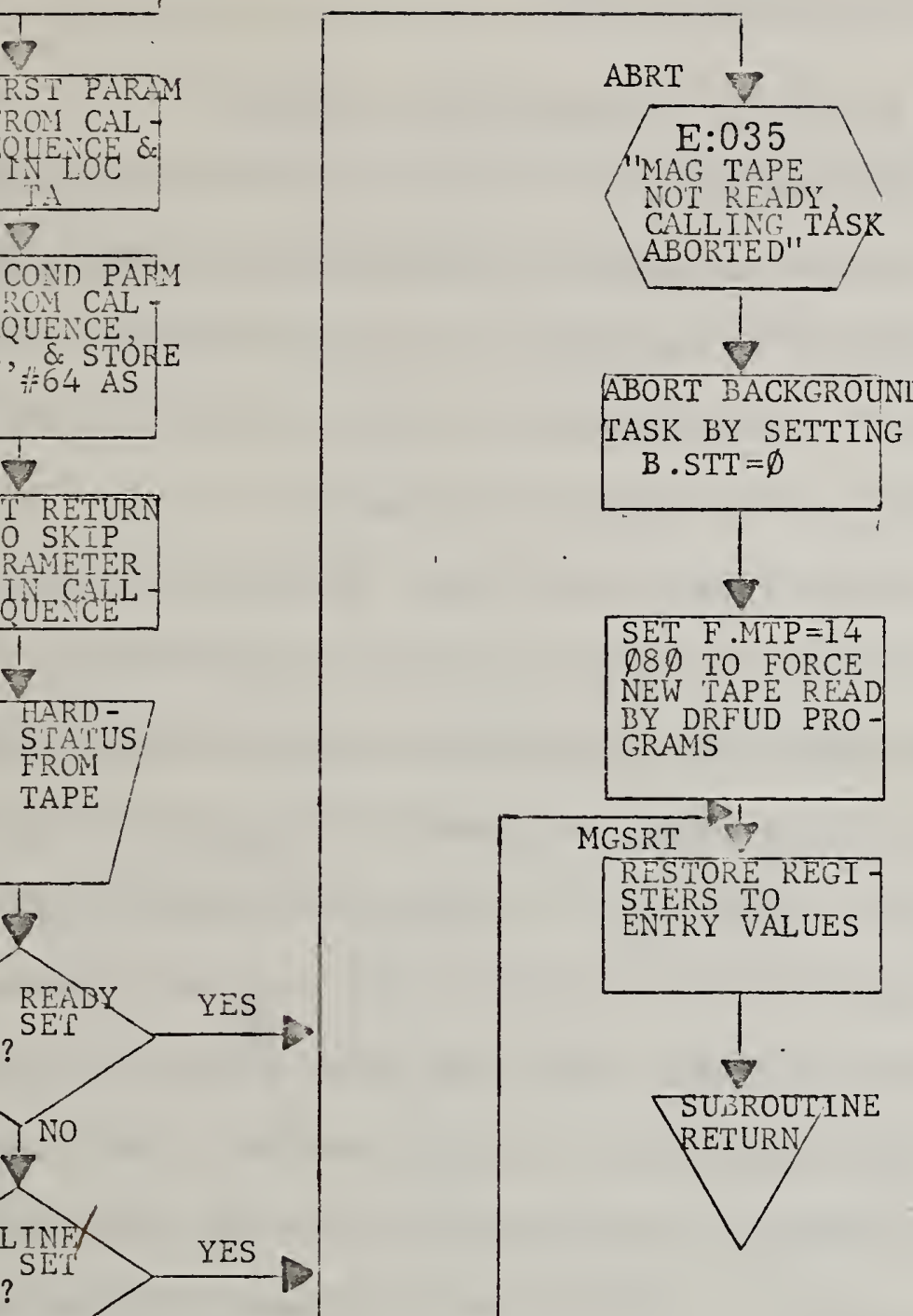

ABORT BACKGROUND

TASK BY SETTING

$B \cdot S T T=\varnothing$

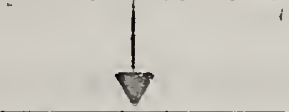

SET F.MTP $=14$

$\emptyset 8 \emptyset$ TO FORCE

NEW TAPE READ BY DRFUD PRO GRAMS

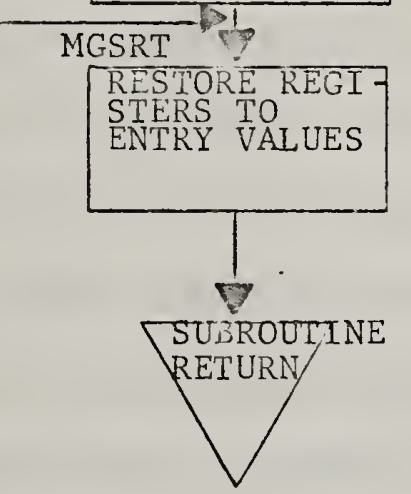


12. U\$RTA

12.1 Functional Description

\subsubsection{U:RTA (U Data Real Time Analys is Routine)}

U:RTA (Real Time Analysis) is the program responsible for maintaining the sensor parameters at optimum operating levels. It is entered from E:SKD after E:UIN flags the scheduler that ten seconds of data have been collected. It performs the following functions with that data from the ten-second time segment just ending:

a. Monitor channel amplitude. Amplitude values between AMPMN and AMPMX (see Figure 12.1) are considered "good" points. Any value below AMPMN is considered low and any value above AMPMX high. These points are tallied in counters called HIVTY (High Value Ially) and LOVTY (Low Value Ially) respectively. Furthermore, points below AMPDR indicate sufficient loss of signal strength to cause the phase value at this time to be considered unusable. The first three amplitude samples during a ten-second time segment which are below the AMPDR level cause the previous second's phase value to replace the current one. This substitution of phase values account for the last second's data from the previous tensecond time segment being saved in the first row of the working buffer-U.WRK (making eleven rows in a11). If more than three values below AMPDR are encountered, a data validity flag, MUBAV, is set to -1 to indicate a signa? lost condition. Of the points greater than AMPMX, those above 
SATUR saturate the sensor, causing the phase estimation to be distorted and therefore unreliable. If more than six points are greater than SATUR during a ten-second time segment, MUBAV is then set to +1 to indicate a saturated signal condition. The last amplitude value from the ten-second time segment is saved for each channel in U.AMP as that channel's amplitude estimate for the ten-second segment.

b. Monitor channe1 phase. When a fifty, microsecond phase shift is induced in the reference frequency of the sensor's mixer, E:UIN (see Section 6.1 .2 (b)) sets a flag bit for the then current phase value to show that the shift would take place before the next sample. The phase monitoring part of U:RTA detects this bit and adds U.CYC (which was set to $\pm 5 \emptyset$ microseconds, depending on the direction of the shift) to U.CCN, which contains the total compensation for the cycle shifts to the moment, before the next point is considered. U.CCN is added to each phase value to create continuity in the data stream over these shifts. This extends the phase range from 100 microseconds at the sensor to about $890 \emptyset$ microseconds at the processor. The average of the current ten-phase values, including the shift compensations, is finally stored for use by the least squares slope algorithm (U:LSQ).

c. Performs data compression. Each data point is compared to the current window maximum and minimum values to determine whether or not it lies within the current window (see Section 12.3.2 for a discussion of the data compression algorithm). If a point falls outside the window, it is established as the center (CENTR) of the new window, around which new maximum (WINMX) and minimum (WINMN) yalues are computed. The window 
size is determined by the standard deviation of the phase values. The new window center is considered a compressed data point, and is held with its time in a buffer (U.CMP) and tallied by a compressed point counter (U.CPC). Each time a compressed point is discovered, bit 11 of the console switch register is examined. If that bit is set, and the binary representation of the sensor channel on which the point was discovered is given in switch register bits $12-15$, then the time and phase value of that compressed point is displayed for the operator on the ASR 35 teleprinter.

The following table relates the sensor channels and the value to be entered into bits $12-15$ of the console switch register for use in specifying which channel's compressed data points are to be displayed on the ASR 35.

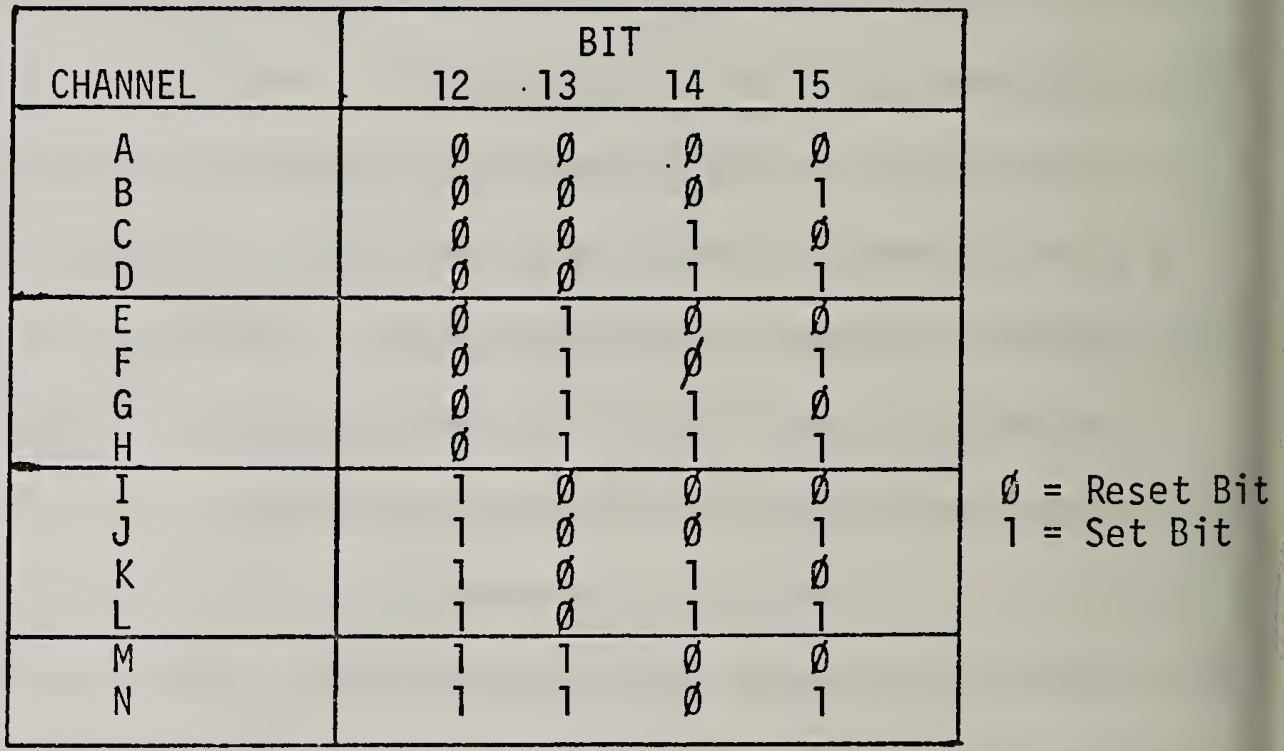

Tab?e 12.1 Compressed Data Point Channel Specification 
d. Monitors percent blanked leve1. The last "percent blanked" value of each ten-second segment is summed (for each channel) over two minutes (i.e. twelve points).

e. Controls channel RF gain. Every two minutes, controlled by the gain clock (GCLOK), the number of points tallied below AMPMN and above AMPMX are compared to a count of 90 (i.e. $75 \%$ of a11 points during that two minute period). If one of these levels exceeds this value, the RF gain is increased or decreased accordingly, and the action is logged on the ASR 28. A11 counters and clocks are reset for the next two minute period.

f. Controls blanking leve1. When the gain control program completes execution, the sum of "percent blanked" values over the previous two minutes is compared against BLKMN and BLKMX (see Figure 12.1) to determine the need to increase or decrease the blanking level. If such a change is made, the action is logged on the ASR 28.

g. Compute standard deviation. In the phase monitoring part of U:RTA, the difference between the individual phase values and the previous ten seconds' average (XBAR) was accumulated. Once every 90 seconds, controlled by the tracking clock (TCLOK), this accumulated sum is fed into the standard deviation algorithm described in Section 12.3. 1 and a standard deviation estimate (SIGMA) is computed.

h. Controls tracking rate. The standard deviation is compared against TRMIN and TRMAX (see Figure 12.1) to determine the need to increase or decrease the tracking rate. If such a change is made, the action is logged on the ASR 28. 


\section{GAIN CONTROL LEVELS}

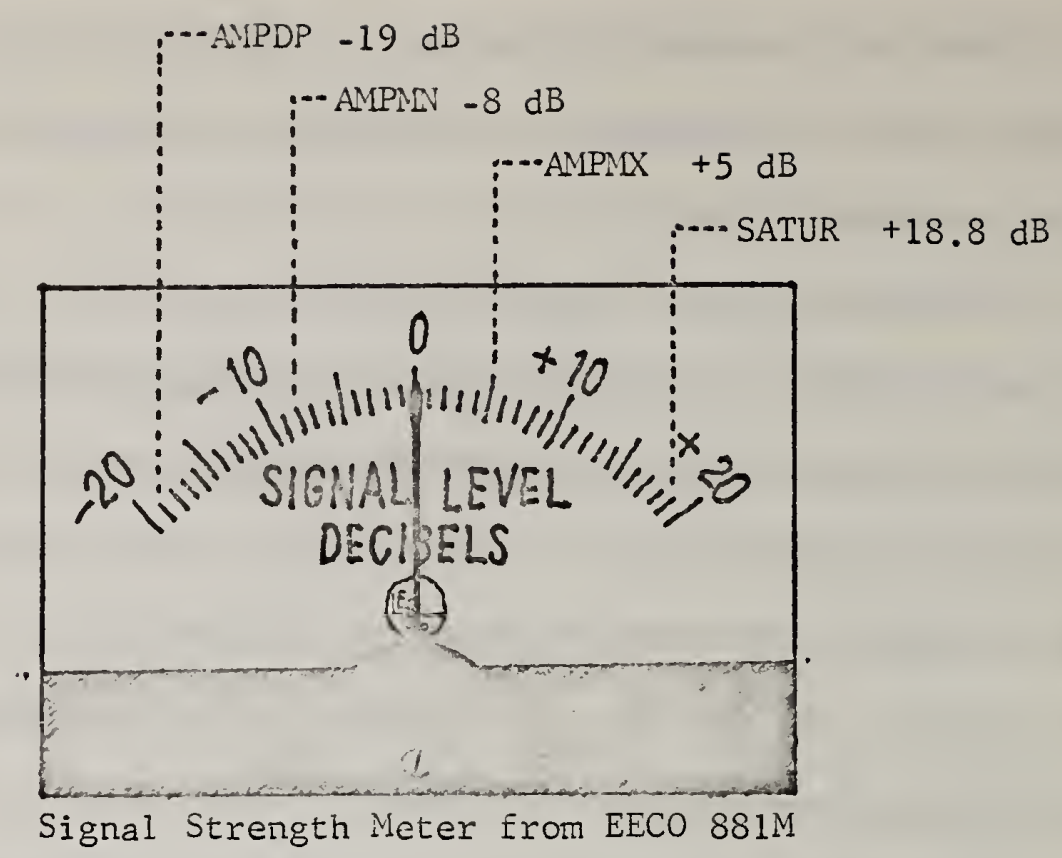

BLANKING RATE CONTROL LEVELS

$4.1 \%$

BLKMN
$4.5 \%$

BLKMX

TRACKING RATE CONTROL LEVELS

Standard Deviation: .5
1.25 microseconds TRMAX

Figure 12.1 Parameter Control Levels 
i. Controls the channel down sequence. When MUBAV is set to \pm , the data during that segment is considered invalid, and the channel is put into, or continues in a down sequence. The channel passes through four states (determined by the value of U.CST):

(1) UP (U.CST=1). In this case, MUBAV $=\varphi$, indicating good data. Waveform processing continues as normal.

(2) COASTING, NO REPORT (U.CST=2). The first two times MUBAV is not $\emptyset$, the channel is put into the first coasting state. The operator is not informed and most waveform processing continues as norma 1.

(3) COASTING, REPORTED (U.CST=3). After thirty seconds of bad data; i.e., MUBAV $\neq \not d$ three times, the channel is put into the second coasting state and the reason for the data being considered invalid is logged on the ASR 28. If MUBAV was -1 a11 three times, the signal will be logged as lost ("CF", channel Coasting for signal Failure). If MUBAV was +1 a 11 three times, the signal will be logged as saturated ("CS", channel Coasting for signal Saturation). If neither of these cases should occur, the channel is logged "CN." In this state, the waveform analysis will be permitted to continue on any signal currently in progress, but new signals will not be started.

(4) DOWN (U.CST $=4$ ). Whenever processing is inhibited by an INE or MAINTENANCE condition (see Section 20), or in a coasting state for five minutes, the channel is put DOWN. While in the DOWN state, no data processing whatsoever is performed on that channel. 
A channel's status will be returned to UP from any state when three minutes pass with all vaiues of MUBAV $=\varnothing$, indicating a continuous stream of good data for $18 \emptyset$ seconds. The amplitude monitoring part of U:RTA performs this movement to the UP state by incrementing the recovery tally (U.RTY) each time it is not zero and MUBAV is zero. When U.RTY reaches zero, the channel is put up and the necessary parameters are reset to permit the data processing to resume on it.

\subsubsection{U:CNI (Signal In Progress Checking Subroutine)}

U:CNI is a subsection of the internal subroutine called CANI. During periods when a valid signal is probably in progress, NUSTAD refrains from making automatic adjustments to the control settings on the sensor. The OPC: option permits the operator to request these adjustments without his knowing the waveform processing status. Therefore, before his requested adjustment is made, U:CNI is called to determine whether or not a signal is in progress. The following criteria are used to establish a signal as in progress:

a. U.AST, the analysis status, indicates a peak has been found.

b. If an onset has been found, more than $5 \emptyset$ seconds have elapsed since that onset.

The answer as to whether a signal is in progress or not is indicated to the calling program by the location where control is returned. If the adjustment is not permitted, control is returned immediately. Otherwise, the location counter is incremented by two before the return. The calling sequence iooks like this: 


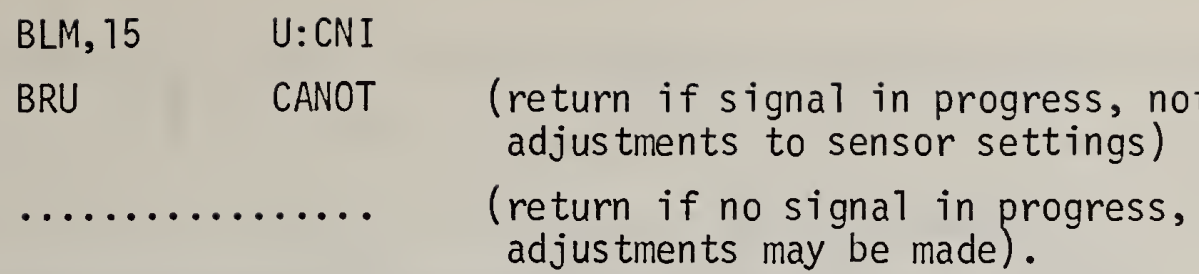

Where channel being checked is indicated in R6.

\subsection{Called Subroutines}

SUBROUTINE

E:DIF

E\$DIF

$E: S R T$

ESSRT

E:MIT

E\$UIN

U:LSQ

U\$LSQ

$E: 035$

$E \$ T 35$

$E: F C T$

E\$FRT

$E: L O G$

$E \$ L O G$

$E: F M F$

E\$FMF
FUNCTION

Establishes whether or not a signal is in progress on a given channel, and if one is, returns the time difference in seconds between onset and the parameter value.

Computes the square root of the parameter.

Transmits sensor parameter settings to equipment.

Least squares slope computation routine, next step in processing current ten-second data segment.

Transfers output message to buffer before transmission to the ASR 35.

Reformats BCD time from memory to ASCII for output.

Generates coded messages for the station $\log$ and initiates their transfer to the ASR 28 via $E \$ T 28$.

Reformats phase values from internal binary representation to ASCII decimal for output. 


\subsection{Computational Techniques}

\subsubsection{Standard Deviation}

NUSTAD uses the standard deviation of the incoming phase values to adjust the tracking rate and to determine the offset necessary to trigger the waveform analysis routine. The standard deviation is computed by a variation on the mathematical definition of the standard deviation. The actual definition for the standard deviation of a set of $n$ elements, call them $\left(x_{\emptyset}, x_{1}, x_{2}, \ldots x_{n-1}\right)$ is:

Where $\bar{x}=\frac{\sum_{i=\emptyset}^{n-1} x_{i}}{n}$

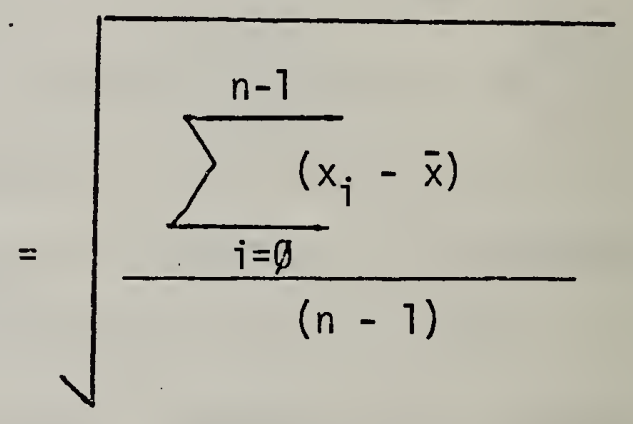

In NUSTAD, the buffering requirement necessary to actually use this approach ( $9 \emptyset$ samples per channel times 14 channels) makes the computation impractical. Therefore, $\bar{x}$ (called XBAR) is approximated by the arithmetic mean of the previous rather than the current $9 \emptyset$ second period. Experience has shown this to be reasonable as the standard deviation is "frozen" during periods of large phase offsets, where this computation would be most affected by such a substitution. The sum of the 
differences between the individual points and XBAR is stored (double precision) in U2ACC; while UACC 1 accumulates the sum of the current points to determine XBAR for the next $9 \emptyset$ second interval. The square root is computed by the executive support subroutine E\$SRT (see Section 28).

\subsubsection{Data Compression}

The data compression algorithm used by INUSTAD is based on a technique called the Zero-Order Predictor Floating Aperture method. The procedures of the technique are:

"In the floating aperture algorithm of the zero-order predictor, an aperture of $2 \mathrm{~K}$ is placed around the last transmitted data point. If each new data point lies within the aperture placed about the last transmitted data point, then the new data points are not transmitted. If a new data point falls outside the aperture about the last transmitted data point, then the present data point is transmitted and the process is repeated. The predicted point in this case is the last transmitted data point with a tolerance of $\pm K$ placed about $i t$. The aperture, then, has the effect of 'floating' with the last transmitted value."

(From: Andrews, CA.; Davies, J.M.; Schware, R.R.; "Adaptive Data Compression," Proceedings of the IEEE, Volume 55, Number 3, March 1967, page 268.)

In NUSTAD we refer to the aperture as the data window and establish its size as \pm DELTA, a variable as defined below. The window therefore, has minimum and maximum values as defined by:

$$
\begin{aligned}
& \text { WINMN }=\text { CENTR }- \text { DELTA } \\
& \text { WINMX }=\text { CENTR + DELTA }
\end{aligned}
$$

Where CENTR is the last point to fall outside a previous data window. We refer to these points as compressed data points and retain them as the only phase values used by the waveform analysis programs. 
The original design was to establish DELTA as a constant times the last computed standard deviation value (SIGMA), with a minimum value of one microsecond. Experiments with this approach were very encouraging, but a major flaw was soon uncovered. The trigger detection algorithm (see Section 14.1 .1$)$ requires a predetermined number of compressed data points in a single direction to establish an event trigger. If a sudden phase excursion occurred near the end of a $9 \emptyset$ second interval for which a new value of SIGMA would be computed, an insufficient number of compressed points may have been read to establish the trigger. The new SIGMA value, however, would have ballooned due to the effect of points significantly apart from XBAR, and the window opened up accordingly. This would naturally reduce the number of compressed points considered, and on several occasions, to the point where the remaining points needed to establish the trigger, or to confirm a potential peak were not detected. To avoid this situation, SIGMA from the previous computation, called LSIGM was used to establish the value for DELTA, ensuring that the phase excursion could not occur in the same interval for which the standard deviation was computed. Therefore, we may summarize that DELTA is determined as follows:

a. If LSIGM, the standard deviation value used to estimate the background noise level, times 1.5 is less than one microsecond, DELTA is set to one microsecond.

b. If LSIGM times 1.5 is not less than one microsecond, DELTA is set to LSIGM times 1.5 . 
c. If a signal is in progress; i.e., U.AST > 1, DELTA will not be adjusted.

In this way, compressed points are collected based on the level of the background noise for that channel. In a very quiet environment, points separated by no less than one microsecond are examined, while greater noise will desensitize the triggering and waveform analys is routines.

\subsection{Data Formats}

See Section 6.2 for a description of data values used by U:RTA. The phase range, however, has been extended by the addition of U.CCN to compensate for the 50 microsecond phase shifts as described above.

The internal representation of phase may be regarded as follows:

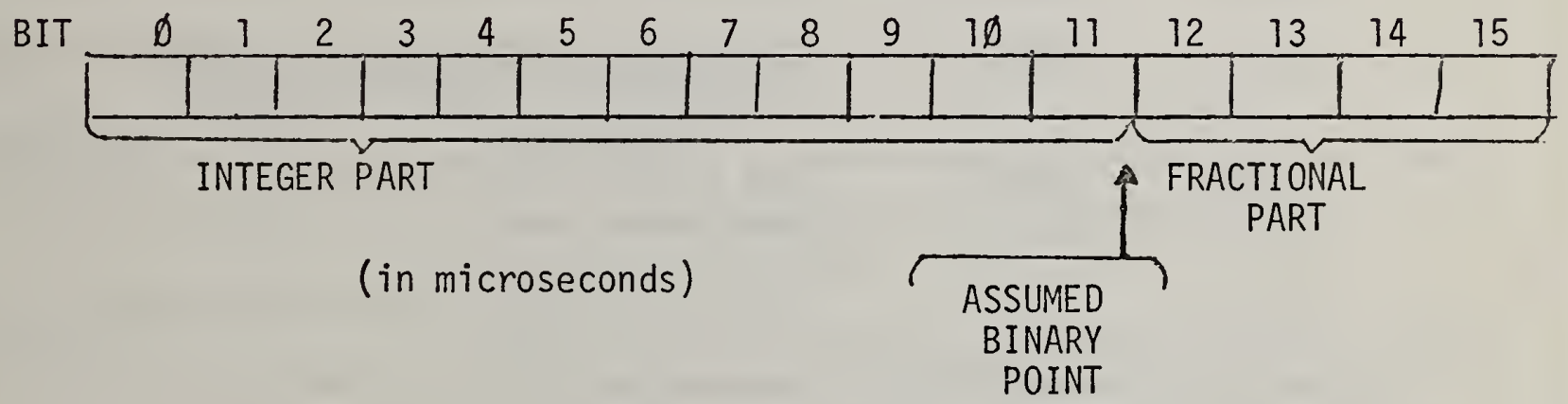

Figure 12.2 Internal Phase Value Representation 


PARAMETER PROGRAM DESCRIPTION

$\begin{array}{ll}\text { U.CYC U\$DAT Set to } \pm 5 \emptyset \text { microseconds when cycle shift is sent } \\ & \text { to the sensor. }\end{array}$

U.WRT U\$DAT Working storage for data from sensor.

U.RTY U\$DAT Channel recovery tally. Counts ten-second segments with good amplitude values to bring channel up after three minutes.

U.IHB U\$DAT Bit $\emptyset$ set to inhibit processing on channel if INE or Maintenance condition set on the channel.

U.AST U\$DAT Channel waveform analys is status, indicates point il signal to which processing has progressed.

U.FAC U\$DAT

The update factor used in the digital filter for the diurnal estimate.

$\begin{array}{lll}\text { U.CCN } & \text { U\$DAT } & \text { Accumulated effect of cycle shifts. } \\ \text { U.LST } & \text { U\$DAT } & \text { Table of ten-second averages used in least squares }\end{array}$ slope computation.

U.LSP R\$DAT

Pointer to next row for storage of points in U.LST.

U.LTF U\$DAT

U.DIU U\$DAT

The longer time constant factor for the digital filter of the diurnal estimate.

U.BLL U\$DAT Current blanking level settings for the channels.

U.TRK U\$DAT

Current tracking rate settings for the channels.

U.GAN U\$DAT

Current RF gain settings for the channels.

U.CPC U\$DAT

Channel compressed point counter.

U.CMP U\$DAT

Compressed point phase and time value table.

U.CTM U\$DAT

Two words of BCD time for the last samples read; i.e., the current time for the ten-second segment. 


\section{5}

Input Parameter Descriptions (continued)

\section{PARAMETER}

U.TRF

$\left.\begin{array}{l}\text { E.TM7 } \\ \text { E.TM2 }\end{array}\right\}$

U.CST

U.AMP U\$DAT

U.CPP U\$DAT

E.IOF $\quad$ E\$T35

M.STA M\$ESC
DESCRIPT ION

Channel trigger flag, indicates status of trigger detecting program.

Two word BCD time used as parameter value for E:FCT

Channel status, used to indicate validity of input data based on amplitude monitoring program evaluation.

Amplitude estimate for current ten-second segment, taken from last second's value of segment for each channel.

Pointers to the beginning of each channel's section of U.CMP.

Parameter word used in calling E:035.

Current values of digital input word status from the sensor.

12.6 Output Parameter Descriptions

PARAMETER SIZE DESCRIPT ION

$U: R T A$

Entry point for Real Time Analysis routine (see Section 12.1.1).

$\mathrm{U}: \mathrm{CNI}$

Entry point for routine to determine whether signal is in progress for granting permission to make sensor parameter setting adjustment. (See Section 12.7.2.)

U.SIG

Standard deviation estimate.

U.SIG is used to external7y access internal parameter SIGMA.

U.TM3

External reference name for the gain clock, GCLOK.

U.TM4

External reference name for the track clock, TCLOK 


\subsection{Internal Data Descriptions.}

PARAMETER SIZE DESCRIPTION

\begin{tabular}{|c|c|c|}
\hline LSTMS & 5 & DOY/TOD group for optional compressed point listing. \\
\hline LSTMG & 7 & Phase value for optional compressed point listing. \\
\hline COSVR & 6 & Register holding buffer. \\
\hline TREGS & 7 & Register holding buffer. \\
\hline AMPMN & 1 & $\begin{array}{l}=6141 \emptyset \text { (equivalent of }-8 \mathrm{~dB} \text { ) minimum amplitude in } \\
\text { "good" range. }\end{array}$ \\
\hline AMPMX & 1 & $\begin{array}{l}=6661 \emptyset \text { (equivalent of }+5 \mathrm{~dB} \text { ) when added to AMPMN, } \\
\text { gives the maximum amplitude in the "good" range. }\end{array}$ \\
\hline AMPDP & 1 & $\begin{array}{l}=5631 \text { ( } \text { (equivalent of }-19 \mathrm{~dB} \text { ) when subtracted from } \\
\text { AMPMN, gives the minimum amplitude in the "valid" ran }\end{array}$ \\
\hline SATUR & 1 & $\begin{array}{l}=7071 \emptyset \text { (equivalent of }+18.8 \mathrm{~dB} \text { ) when added to } A M P M X \\
\text { gives the maximum amplitude in the "valid" range. }\end{array}$ \\
\hline LOVTY & 14 & Count of amplitude values below AMPMN (2 minutes). \\
\hline HIVTY & 14 & Count of amplitude values above AMPMX (2 minutes). \\
\hline SATTY & 1 & Count of amplitude values. above SATUR ( $1 \emptyset$ seconds). \\
\hline DRPTY & 1 & Count of amplitude values below AMPDP ( $1 \varnothing$ seconds). \\
\hline MUBAV & 1 & $\begin{array}{l}\text { Amplitude validity flag: } \\
+1 \text { if amplitude indicates saturated data } \\
\emptyset \text { if amplitude indicates valid data } \\
-1 \text { if amplitude indicates lost signal }\end{array}$ \\
\hline
\end{tabular}

UBADT $\quad 14$

Counter of ten-second segments during which MUBAV $\neq 0$.

UBADS $\quad 14$

Sum of MUBAV values during times of "bad" amplitude.

UACC1 28

Double precision sums of current phase values with cycle shift compensation included.

U2ACC

Double precision sums of the difference between currer phase values and the average phase value of the previo $9 \emptyset$ second interval (i.e., XBAR)

Computed standard deviation of incoming phase values. (See Section 12.3.1.) 
12.7 Internal Data Descriptions (continued)

PARAMETER SIZE DESCRIPTION

LSIGM $\quad 14 \quad$ Value of SIGMA during previous 90 second interval.

XBAR 14 Average phase value during previous $9 \emptyset$ second interval, used as average phase value in standard deviation computation (see Section 12.3.1).

WINMX $\quad 14 \quad$ Upper bounds for current data windows of the data compression algorithm (see Section 12.3.2).

WINMN $\quad 14 \quad$ Lower bounds for current data windows of the data compression algorithm (see Section 12.3.2).

DELTA

One-half the current window width, minimum value $=1 \mathrm{msec}$. WINMX $=$ CENTR + DELTA WINMN $=$ CENTR - DELTA

K , 2

Double precision constant $=1.5$. Used to compute DELTA; DELTA $=K *$ LSIGM

UBACC

14

Sums last percent blanked value of each ten second segment over a two minute interval.

CFLAG

1

Bit $\emptyset=1$ if an adjustment is needed for the gain or blanking ievel on the current channel.

BLKMN 1

$=\mathrm{B} 9_{16}$, minimum acceptable blanking percentage, $=4.1 \%$.

BLKMX

$=A 9_{16}$, maximum acceptable blanking percentage, $=4.5 \%$

GCLOK

Counts ten-second time segments for two minutes to control entry to gain and blanking level control sections of the program.

TCLOK

Counts ten-second time segments for $9 \emptyset$ seconds to control entry to the tracking rate control section of the program.

TRMAX

$=14_{16}$ (equivalent of 1.25 microseconds) maximum acceptable value of SIGMA for a good tracking rate setting.

TRMIN

$=\emptyset 8_{16}$ (equivalent of .50 microseconds) minimum acceptable value of SIGMA for a good tracking rate setting.

The center of the current data window, also the last compressed data point encountered for each channel. 
U\$PTA

U:R TA

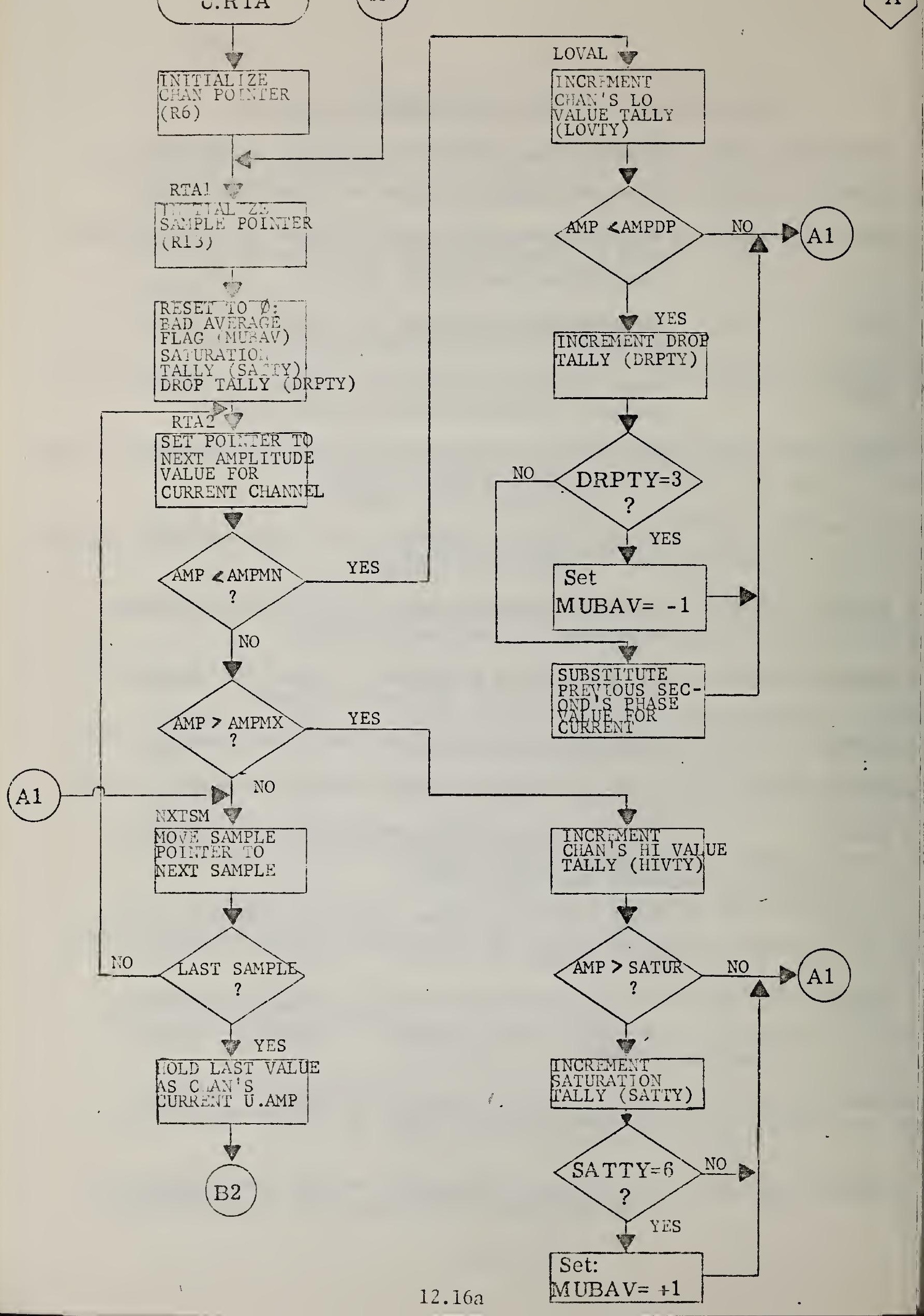




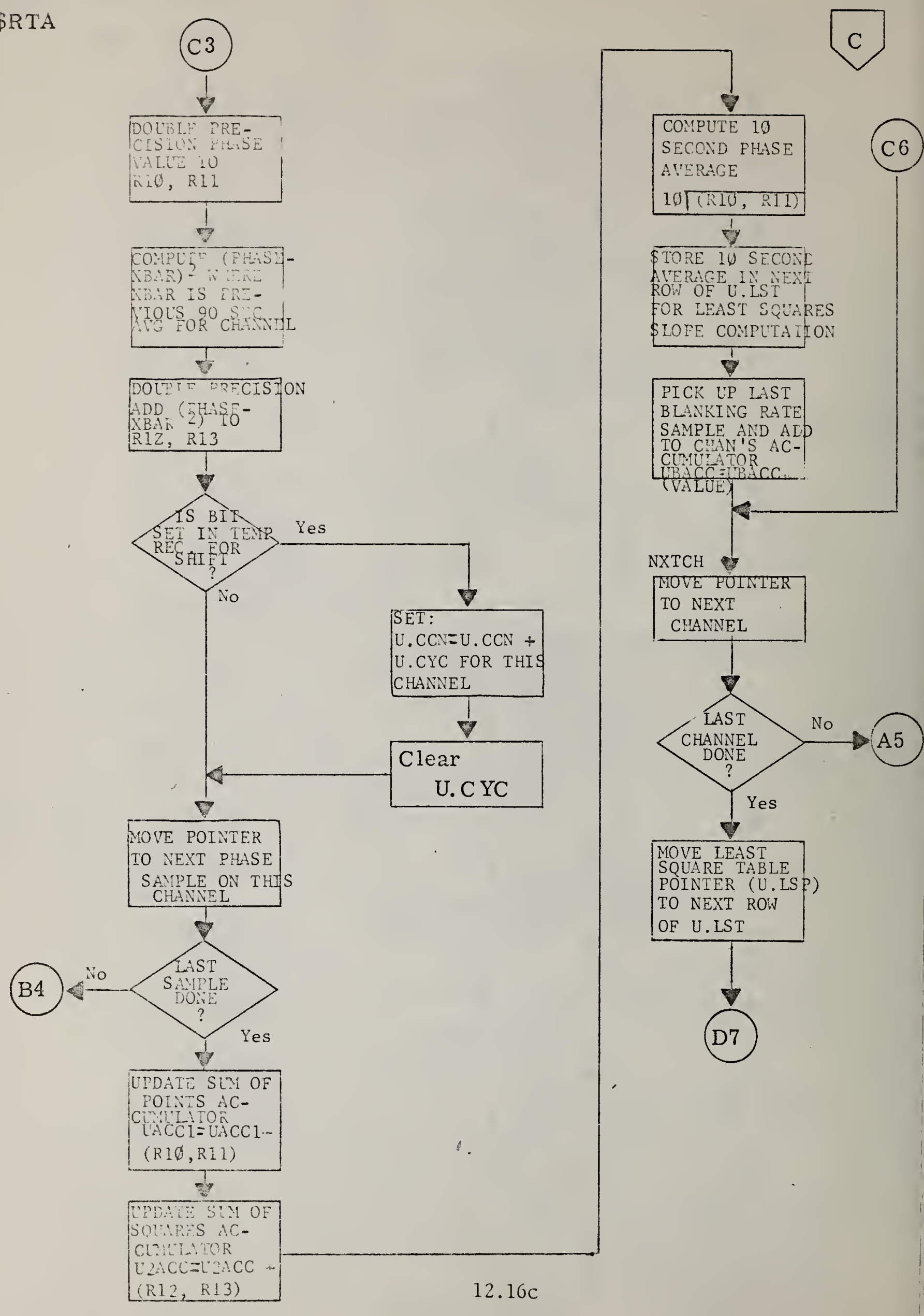




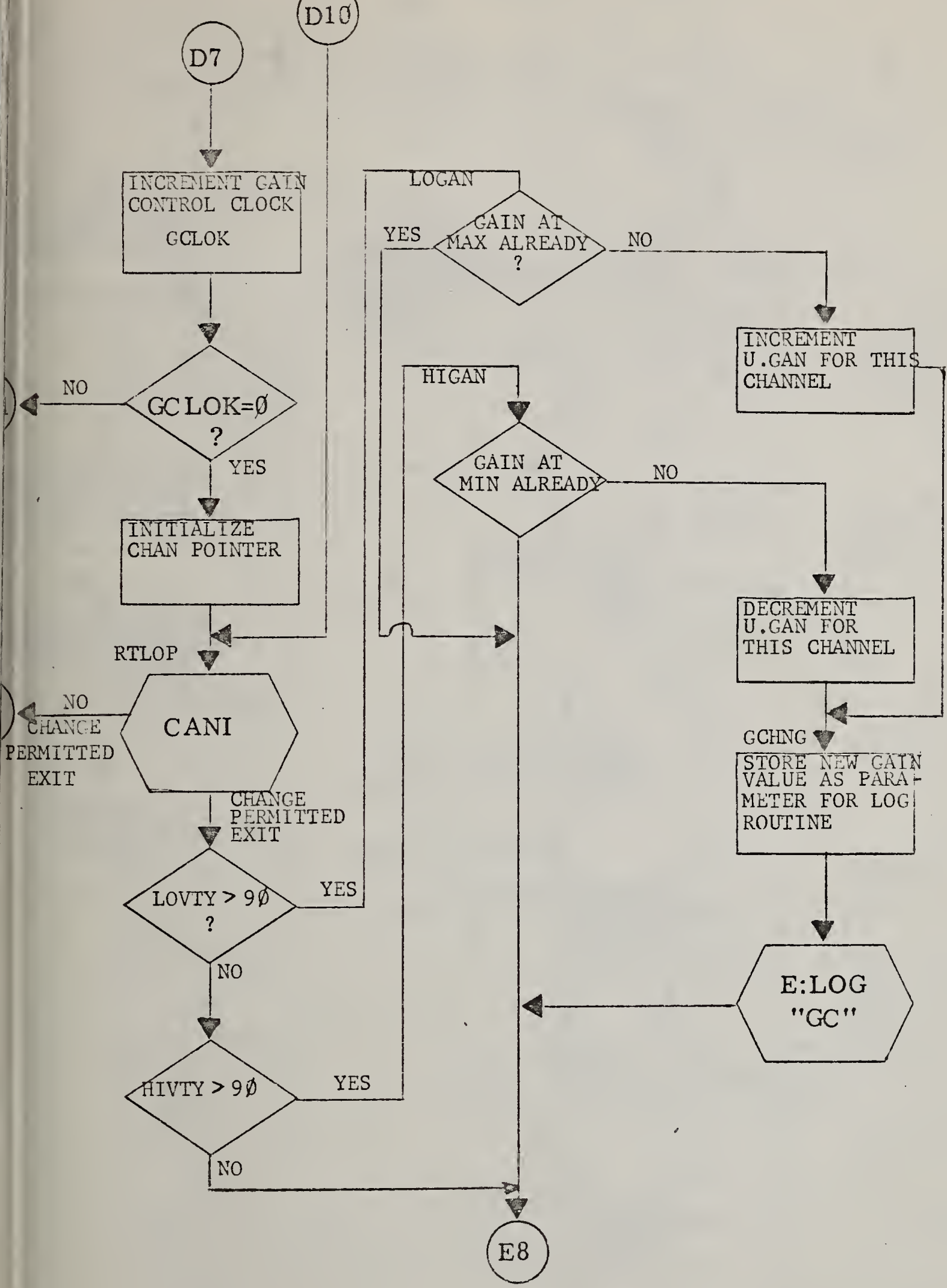




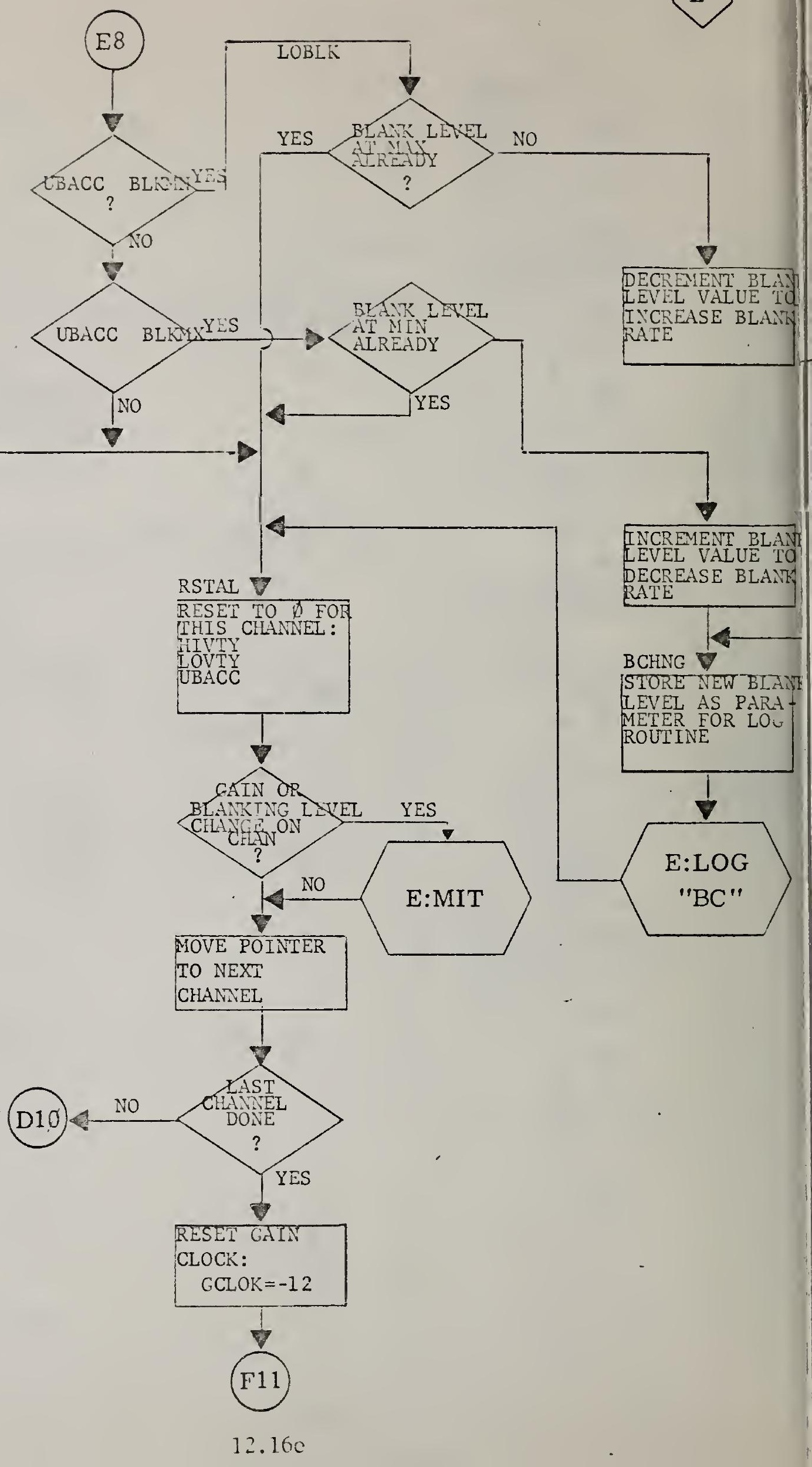




\section{$\mathrm{TCLOK}=0$}<smiles>CC1CCCCC1</smiles>

sio

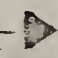

FINI

YOVE LAST ROW

OF U.WRK TO THE

FIRST TO SUPPLY

POINTS FOR GAIN

MONITOR SECTION
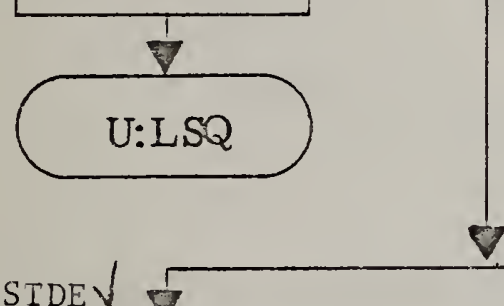

CC गएTाप:

SIGM: $=\sqrt{\frac{\text { UZACC }}{89}}$

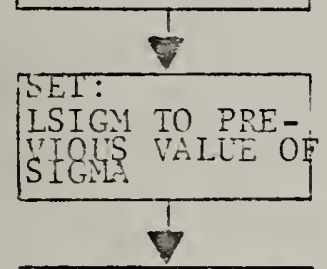

SET:

$\mathrm{XBAR}=\frac{(\mathrm{VACC})}{9 \varnothing}$
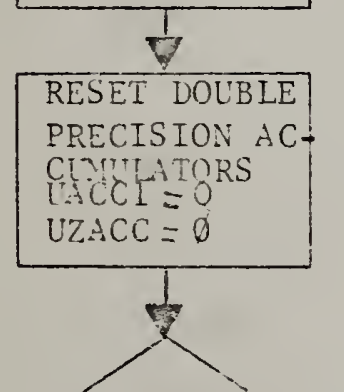

U.AST $>1$ Yes

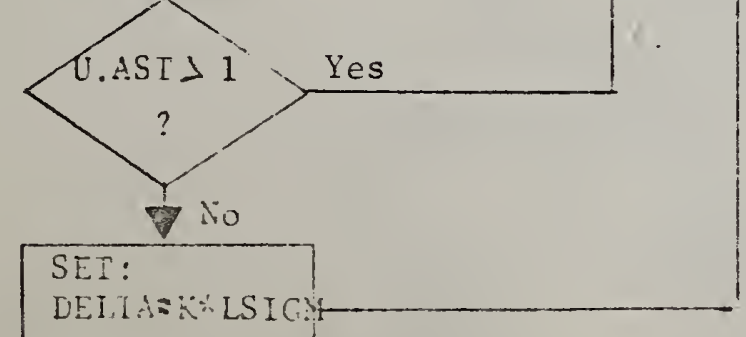

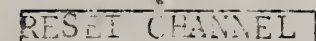
POINTER TO FIRST 


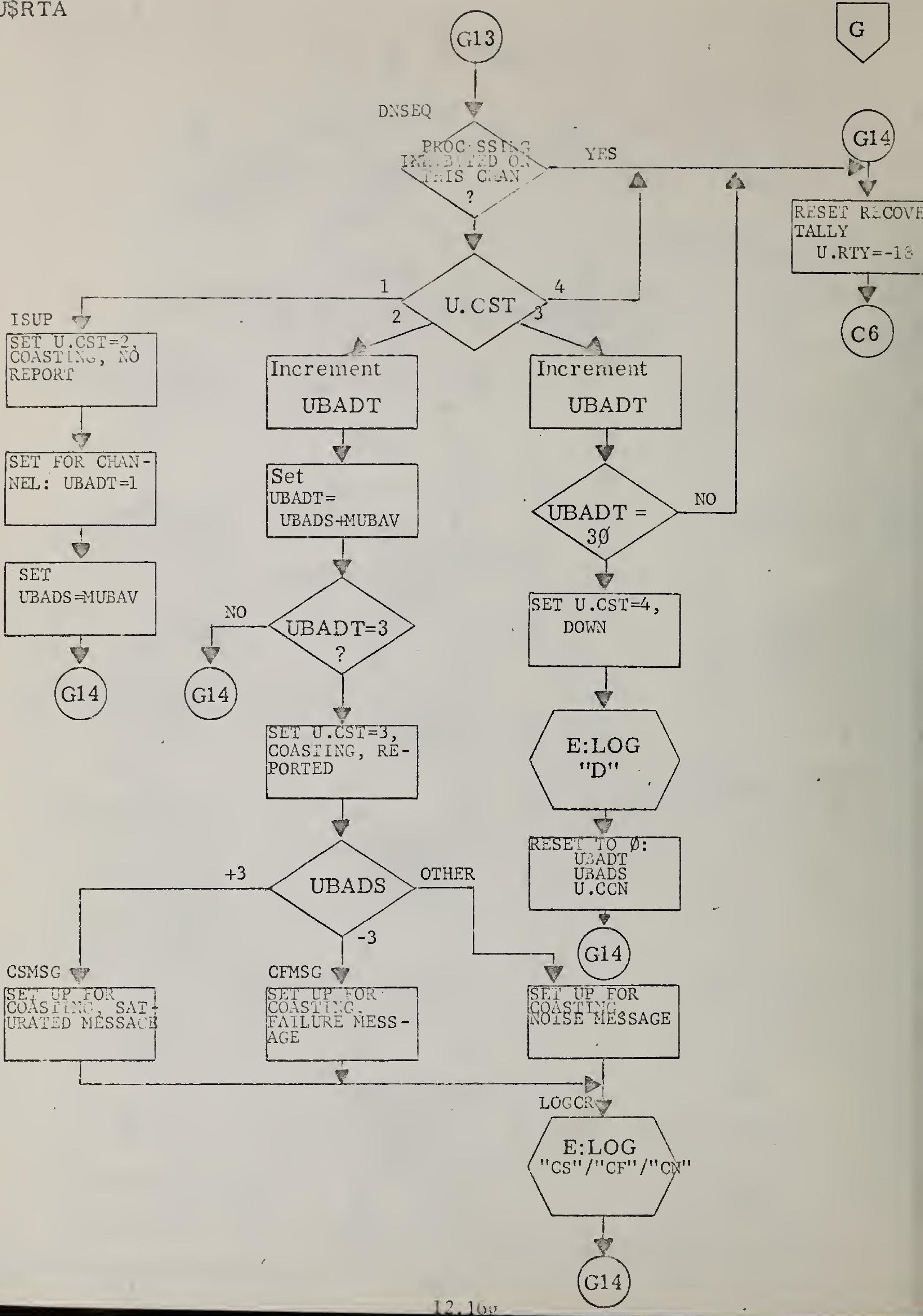




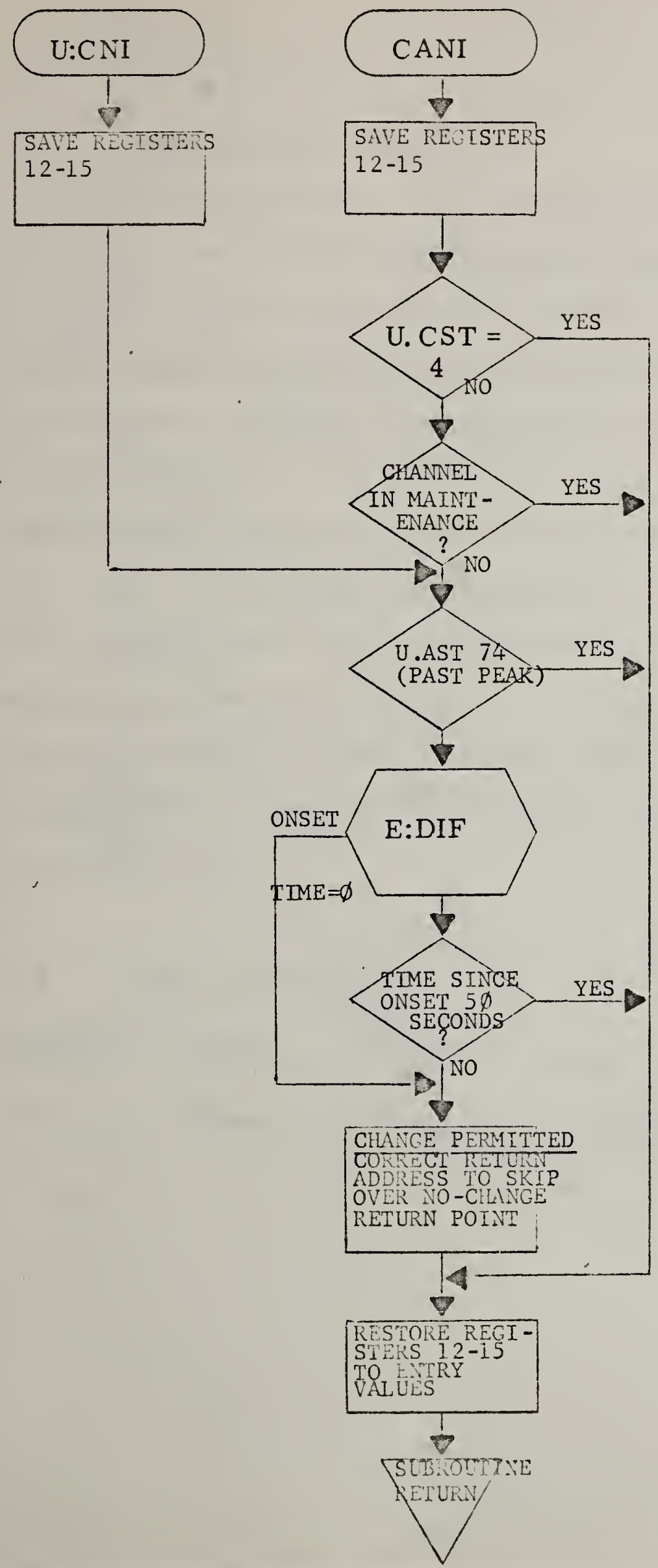




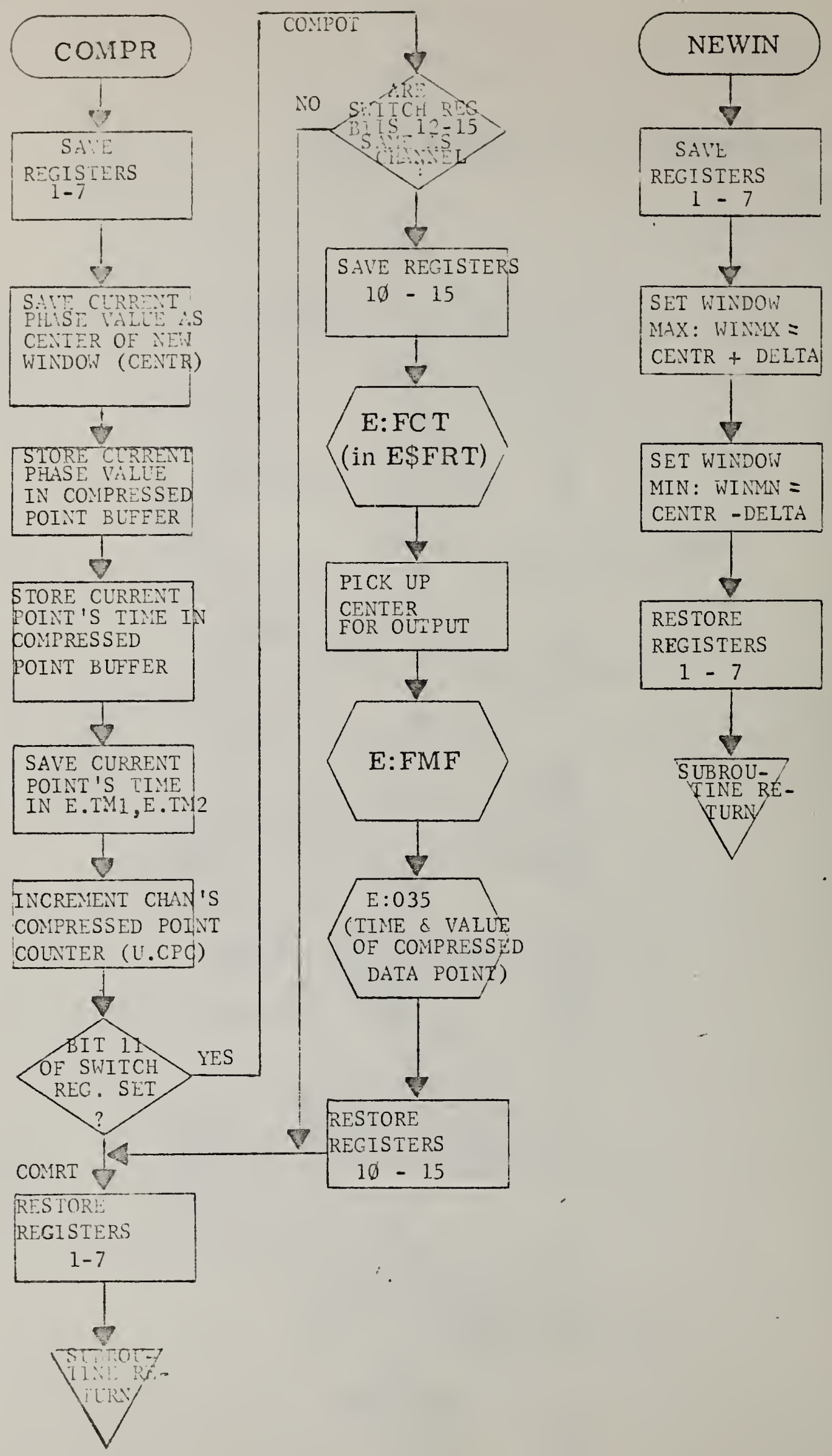


13. U\$LSQ

13.1 Functional Descriotion

Two parameters are used by the trigger detection and waveform analysis programs (USTGR and USWFA) in processing $U$ data signals in real time. While selected phase values are stored for use by USRTA in its data compression scheme (see Section 12.3.2), the waveform analysis algorithm used by NUSTAD also relies on information regarding the trends in the phase data. The values used to determine these trends are derived from the short term estimates of the phase slope. NUSTAD uses a Least Squares Estimation as a maximum likelihood fit to determine this short term ( $1 \varnothing \emptyset$ second) slope value. Each ten seconds, after U\$RTA passes control to U:LSQ, the slope estimate for the preceding $\$ \varnothing \emptyset$ seconds is computed and stored in U.SLP for for each channel. When completed, control is passed to U\$TGR for trigger detection.

\subsection{Called Subroutines}

SUBROUTINE PROGRAM FUNCTION

U:TGR U\$TGR Trigger detection routine, next step in processing current ten-second data segment. 


\subsection{Computational Methods--Least Squares Algorithm}

The short term slope estimate is made by NUSTAD by applying a least squares fit to ten-second segment average phase values over a period of 100 seconds. The formula for such a slope estimate is:

$$
\text { SLOPE }=\frac{\Sigma T_{n} \Sigma Y_{n}-K \Sigma T_{n} Y_{n}}{\left(\Sigma T_{n}\right)^{2}-K \Sigma T_{n}^{2}}
$$

Where: $K$ is the number of average values considered, currently $=10$ (i.e. ido seconds)

The sums are taken between the limits of $n=1$ and $k$ $T_{n}$ is the time value (in seconds.) for the $n^{\text {th }}$ average value $Y_{n}$ is the 10 second phase average at time $T_{n}$

Since the actual value of $T_{n}$ is immaterial to the computation, and samples are taken at uniform intervals, assume the interval value $A=10$ seconds and assign $T_{1}=$ zero. Then we can determine $T_{n}$ as follows:

$$
T_{n}=A(n-1)
$$

Then it follows:

$$
\begin{aligned}
& \Sigma T_{n}=\frac{A K(K-1)}{2} \\
& \Sigma T_{n}^{2}=\frac{A^{2} K^{2}(K-1)(2 K-1)}{6}
\end{aligned}
$$

Substituting these values in (1), we determine:

$$
\text { SLOPE }=\frac{\frac{A K(K-1) \Sigma Y_{n}}{2}-A K \Sigma(n-1) Y_{n}}{\frac{A^{2} K^{2}(K-1)}{4}-\frac{A^{2} K^{2}(K-1)(2 K-1)}{6}}
$$


Which simplifies as follows:

$$
\begin{aligned}
\text { SLOPE } & =\frac{\frac{A K(K-1) \Sigma Y_{n}-2 A K \Sigma(n-1) Y_{n}}{2}}{\frac{3 A^{2} K^{2}(K-1)-2 A^{2} K^{2}(K-1)(2 K-1)}{12}} \\
\text { SLOPE } & =\frac{6 A K\left[(K-1) \Sigma Y_{n}-2 \Sigma(n-1) Y_{n}\right]}{\left.A^{2} K^{2}(K-1)[3 K-1)-2(2 K-1)\right]} \\
\text { SLOPE } & =\frac{12\left[\Sigma(K-1) Y_{n}-\Sigma(n-1) Y_{n}\right]}{2}
\end{aligned}
$$

Since our selection of the starting point is immaterial, a unit adjustment in $n$ (i.e., iet $n=n-1$, changing the limits of summation from $n=\emptyset$ to $k-1$ ) wi11 not affect the computation:

$$
\begin{aligned}
\text { SLOPE } & =\frac{-12\left[\frac{\Sigma-(K-1) Y_{n}}{2}{ }^{2}=\Sigma n Y_{n}\right]}{-A K\left(K^{2}-1\right)} \\
\text { SLOPE } & =\frac{12}{A K\left(K^{2}-1\right)} \sum_{n=0}\left[n \frac{-(K-1)}{2}\right]^{Y_{n}}
\end{aligned}
$$


The leading factor is a series of constants, where:

$A=1 \emptyset$ seconds (the interval between averages)

$K=1 \emptyset$ intervals (resulting in a $19 \emptyset$ second slope estimate)

These values are combined with a scaling adjustment to give the slope in microseconds/minute (at equation (10)) the result is in microseconds/ second) reducing the leading factor to:

$$
\frac{12 * 60}{10 * 10 * 99}=\frac{720}{99 \varnothing \varnothing}=.0727272
$$

The expression in brackets ranges from $-\frac{(K-1)}{2}$ to $+\frac{(K-1)}{2}$ in unit steps, or the set of points $(-4.5,-3.5,-2.5, \ldots \ldots+2.5,+3.5$, $+4.5)$ for the ten intervals currently used by NUSTAD.

To simplify internal representation of these constant values (primarily eliminating the need for both integer and fractional representation causing mixed multiplications which result in wasted time for a real-time system), the leading factor is multiplied by ten, and the factor in the brackets is divided by ten (this also results in steps of . 10 rather than one between the bracket factors). In NUSTAD, these values are given the following names:

$\begin{aligned} \text { TUFAC }= & .727272 \text { (the leading factor, adjusted) } \\ \text { TUMAX }= & .45 \text { (the maximum value for the bracket expression } \\ \text { adjusted } & \\ \text { TUWDF }= & \begin{array}{l}10 \text { (the incremental step between successive values } \\ \text { for the brackets expression adjusted) }\end{array} \\ \text { TUWAT } & \begin{array}{l}\text { The variable which corresponds to the brackets } \\ \text { expression, assuming the values of }-.45,-.35, \\ \\ \end{array}\end{aligned}$




\subsection{Data Formats}

\subsubsection{U.LST and U.LSP}

To compute a new least squares slope estimate at each ten-second interval, it is necessary to have the previous ten-phase averages available for each channel. This results in a 10 by 14 word matrix called U.LST. To eliminate a lot of data shuffling within the matrix, it is designed to be wrapped-around, using a pointer called U.LSP to indicate where the "oldest" values are located. This is convenient as the U.LSP also indicates where the next values are to be stored, giving double duty to the single value. U.LST may be pictured as shown in Figure 13.1. U.LSP can be represented as $n * 14$, where $n$ ranges from $\emptyset$ to 9 , corresponding to the relative location of the "oldest" average value ( $Y_{\emptyset}$ in the derivation of Section 13.3) for channel A.

CHANNEL

\begin{tabular}{|c|c|c|c|c|c|c|c|}
\hline & & A & B & c & D & $E$ & ..... \\
\hline & $\mathrm{T}_{\mathrm{I}}$ & U.LST & U.LST+1 & U.LST+2 & U.LST+3 & U.LST+4 & $\ldots \ldots$ \\
\hline & $T_{\text {II }}$ & U.LST+14 & U.LST+15 & U.LST+76 & U.LST+17 & U.LST+18 & . \\
\hline RELATIVE & $T_{\text {III }}$ & U.LST+28 & U.LST+29 & U.LST $+3 \phi$ & U.LST+31 & U.LST+32 & $\cdots$ \\
\hline TIME & . & . & & & & & \\
\hline SEPARATED & . & . & & & & & \\
\hline BY TEN & . & . & & & & & \\
\hline ECONDS & . & - & & & & & \\
\hline & $\mathrm{T}_{\bar{X}}$ & U.LST+126 & U.LST+127 & U.LST +728 & U.LST+129 & U.LST+130 & ...... \\
\hline
\end{tabular}

Figure $13.1 \quad$ Structural Organization of U.LST 


\subsubsection{TUWAT, TUWDF, TUMAX, and TUFAC}

The interval values of USLSQ which represent totally fractional values are represented in the following format:

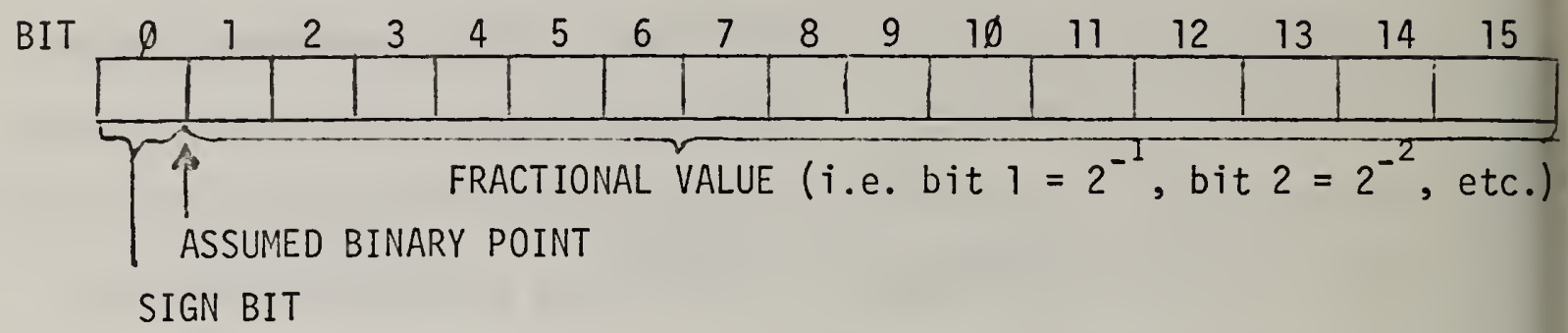

Figure 13.2 Internal Representation of Fractional Values

\section{$13.4 .3 \quad$ U.SLP}

The computed value of slope is stored in U.SLP as a value in microseconds per minute, and may be interpreted as follows:

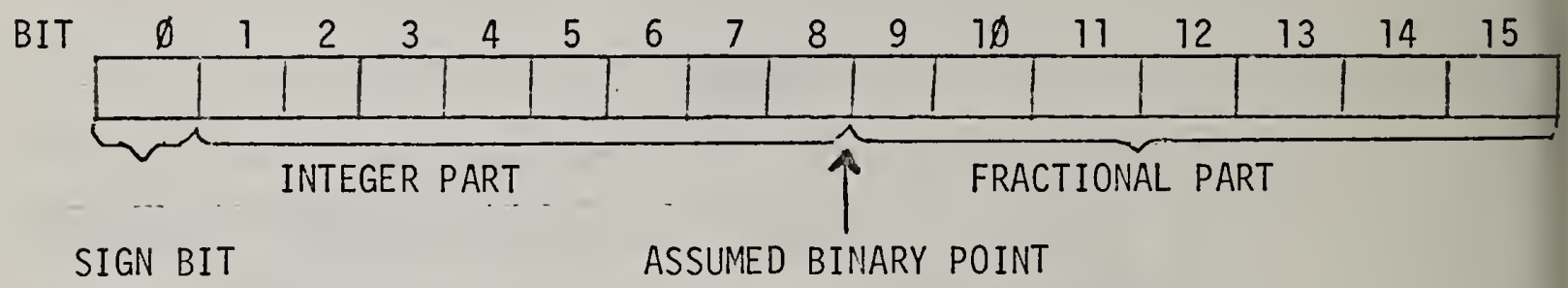

Figure 13.3 Representation of Slope in U.SLP 


\subsection{Input Parameter Descriptions}

PARAMETER PROGRAM DESCRIPTION

U.LST U\$DAT Table of ten-second phase averages for last $7 \varnothing \emptyset$ seconds, see Section 13.4 for description.

U.LSP U\$DAT Pointer to base of oldest row in U.LST (see Section 13.4)

U.CST U\$DAT Channel status, used to indicate validity of input data based on amplitude monitoring program evaluation.

U.SLP U\$DAT Computed slope estimate in microseconds per minute. Indicates short term data trends.

13.6 Output Parameter Descriptions

PARAMETER SIZE DESCRIPTION

U.LSQ Entry point for least squares slope computation routine (see Section 13.1).

13.7 Internal Data Descriptions

PARAMETER SIZE DESCRIPTION

SUM

28

Double precision accumulator for the term

$$
n-\frac{(K-1)}{2} Y_{n} \quad \text { in equation (1) of Section 13.3. }
$$

TUWAT

The "brackets expression" value (see Section 13.3).

TUWDF

$=\emptyset \operatorname{ccc}_{16}=.1 \emptyset_{1 \emptyset}$; the incremental step value for the "brackets expression" of equation (10), Section 13.3.

TUMAX

1

$=3999_{16}=.45_{10}$; the absolute value of the extreme limits of the "brackets expression" of equation (19), Section 13.3.

TUFAC

$=50177_{16}=.727272$; the "leading factor" of equation (ip), Section 13.3. 


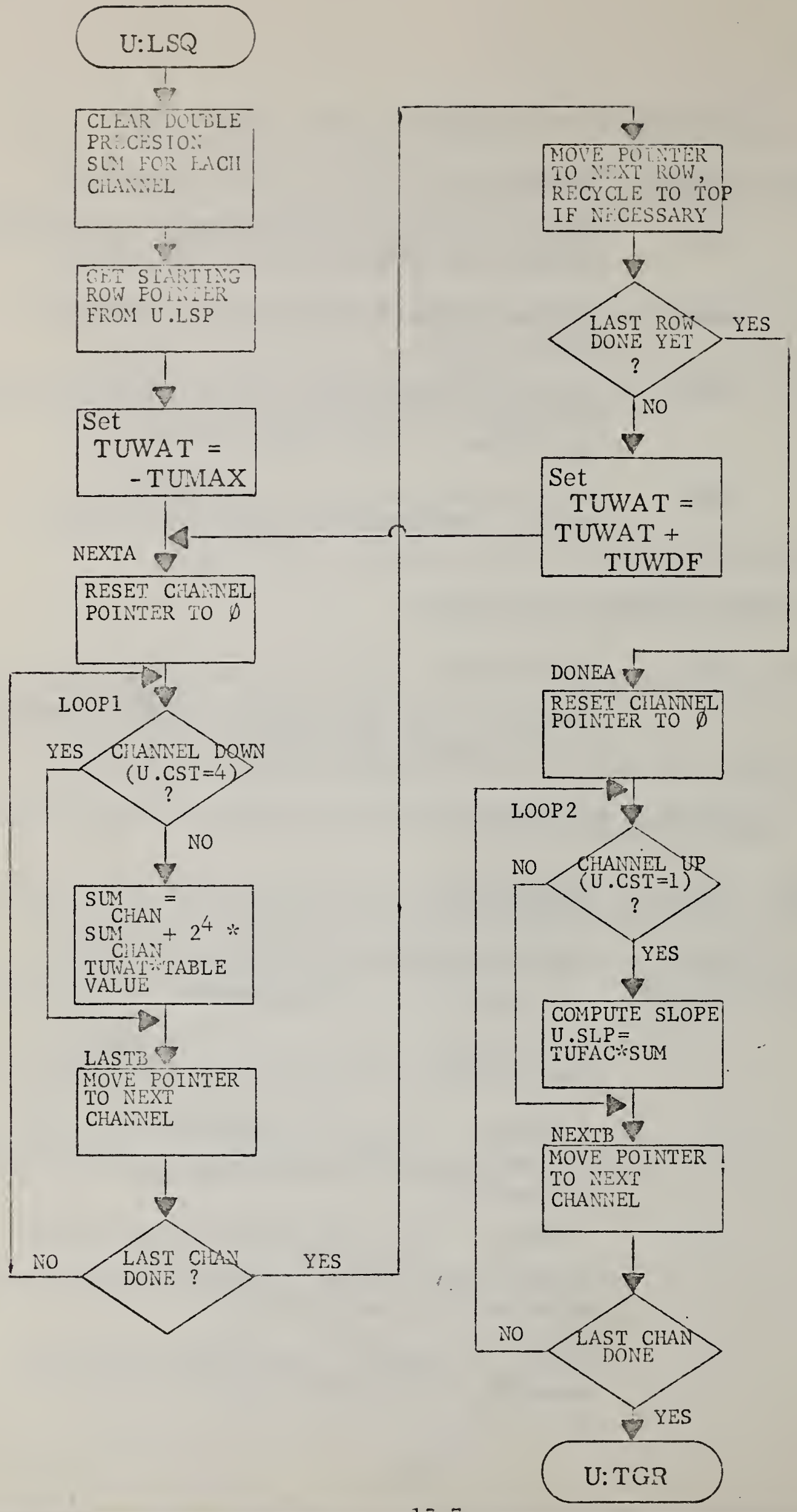

$13.7 \mathrm{a}$ 
14. USTGR

14.1 Functional Description

\subsubsection{U.TGR (U Signal Trigger Detection Routine)}

After the sensor parameter control is completed by U:RTA, and the values needed by the waveform analysis programs are determined by U:RTA and U:LSQ, U:LSQ passes control to U:TGR to begin signal processing. The waveform analysis consists of recognizing key characteristics of the incoming waveform, computing descriptive parameter values based on data values at the key points and comparing the computed values against predetermined criteria.

The initial problem in the waveform analys is algorithm development was to readily recognize a significant phase excursion from the long term trend (see Figures 15.1, 15.2, and 15.3 for the onset portion of the waveforms). The technique finally chosen was to designate a predetermined number of compressed data points in the same direction as the signal onset. NUSTAD uses the standard deviation of the incoming signal to determine the window width in the data compression algorithm and thus effectively eliminating the effects of the background noise in signal processing. The selection of compressed points which consistently increase or decrease becomes a fair indication of short term monotonic trends. The number of points required to recognize an onset, also known as triggering the waveform (hence U:TGR), is stored 
in U.TRL (the $\underline{U}$ Signal Trigger Limit). This value is normally set as five, but may be reset to other values during other portions of the waveform processing. Since the phase excursion should be somewhat sudden, NUSTAD requires that the time difference between the first and last points in the triggering sequence be less than TRTIM (Trigger Time) seconds, currently set at $3 \emptyset$.

The progress in the waveform analys is is reflected by the value of a parameter called U.AST ( $\underline{U}$ Signal $\underline{A}$ larm Status). The values which this variable may take are represented graphically in Figures 15.1, 15.2, and 15.3 for the three signal types which must be recognized. While seeking an onset, U.AST will be set to one. When U:TGR identifies an onset, it sets U.AST to either two or three, reflecting the direction of the phase excursion and in so doing, triggers further waveform processing by U:WFA (U Signal Waveform Analysis).

The status during the trigger detection process is maintained in a

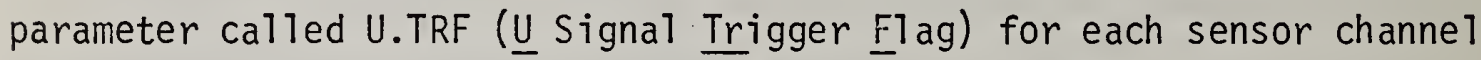
and takes on the following values:

a. U.TRF $=\emptyset$. No compressed data points have been encountered yet. This value can occur only upon initial startup, or after a channel comes up, as thereafter, compressed data points will always have occurred at sometime in the past.

b. U.TRF $=1$. One compressed data point has been encountered. Again, this value will oniy occur as a channel comes up. Since two points are required to determine direction, nothing significant can 
happen until a second point arrives. At that time, the direction is determined and U.TRF set to two or three to indicate an advance or retard trend, respectively.

c. U.TRF $=2$. Two or more compressed data points have been encountered, each point with a phase value numerically smaller than the previous. NUSTAD considers these points as being in the ADVANCE direction. The number of points currently encountered in the possible triggering sequence is contained in TRCNT (Trigger Counter). As long as points continue the decreasing value trend, U.TRF will remain set at two, unti1 TRLIM (Trigger Limit) points have been counted. At this time, U.TRF may be set to four, after additional processing as described in paragraph (e) below.

d. U.TRF $=3$. U.TRF is set to three when two or more compressed data points have been encountered, each point with a phase value numerically greater than the previous. NUSTAD considers these points as being in the RETARD direction. U.TRF set to three is the counterpart of U.TRF set to two for data trends in the opposite direction. See Figures 15.1 and 15.2 for this contrast.

e. U.TRF $=$ 4. When TRCNT reaches TRLIM, sufficient data have been encountered to trigger the waveform analysis. However, at this point, the timing requirement is checked by comparing the time differences between the first and last points against TRTIM. If this check is successful, the trigger is considered valid, U.TRF is set to four, and U.AST is set to the previous value of U.TRF (either two or three, 
indicating the type of signal under consideration). On the other hand, if the time difference is greater than TRTIM, the points are "slipped," making the previous second point the current first, and TRCNT one short of reaching TRLIM. If the next compressed point is again in the same direction, the time duration wi11 be checked on the new first and last points.

If at any time after U.TRF reaches two or three, a point is encountered which is in the opposite direction from that under consideration, the value of U.TRF is reversed to three or two, respectively, and the two most recent points are established as the first two in the new potential triggering sequence.

When discussing the compression algorithm, Section 12.3.2, we said that a tolerance of \pm DELTA is placed about the last value used. For the timing and phase values at onset, NUSTAD uses the first point in the triggering sequence for the "onset phase value," and the time of the second point as the "onset time." Figure 14.1 wi11 intuitively explain the rationale behind using these values. Remember that DELTA is assigned a minimum value of one microsecond and typicalty won't significantly exceed this. 


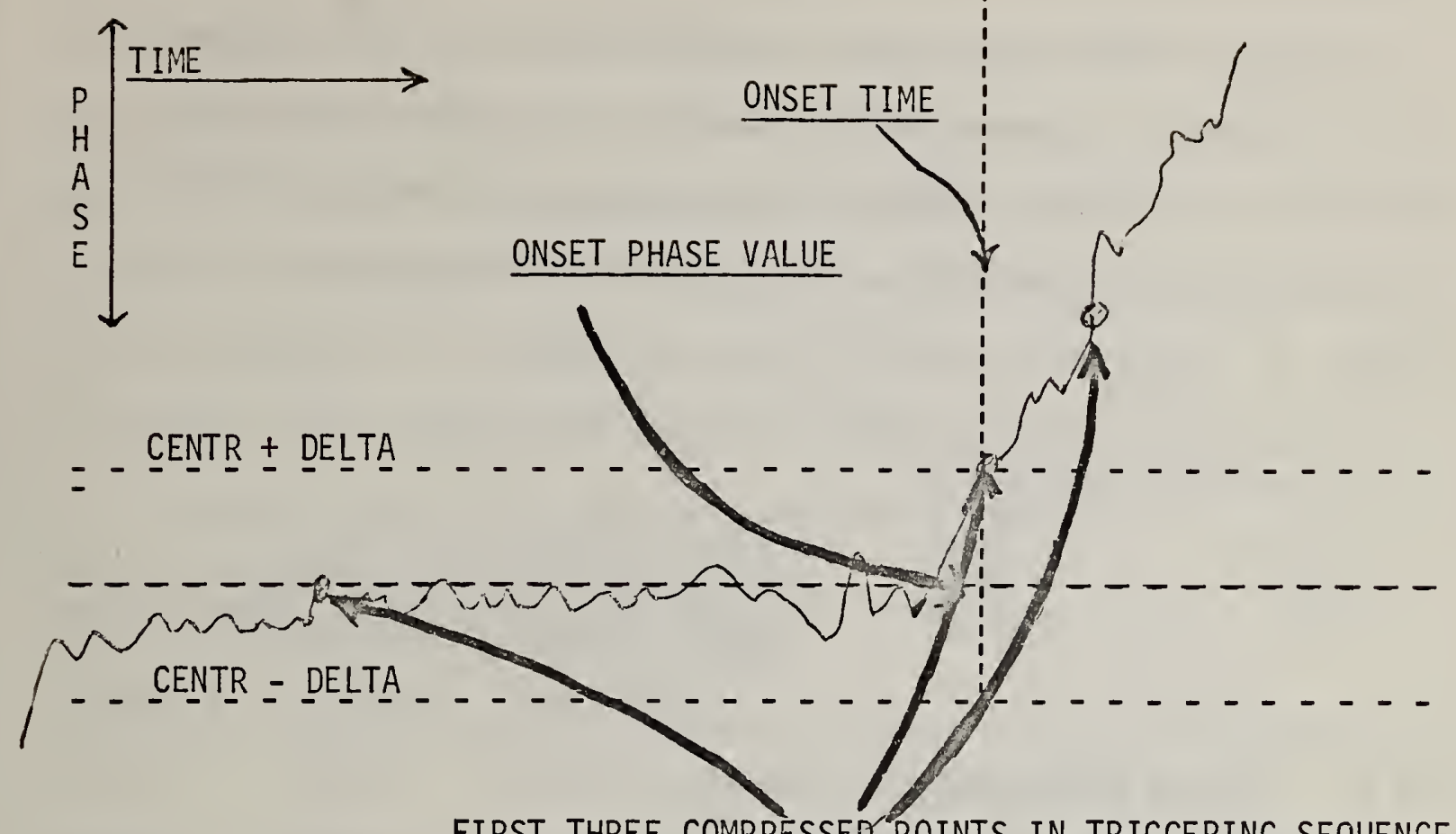

FIRST THREE COMPRESSED'POINTS IN TRIGGERING SEQUENCE

Figure 14.1 Onset Value Determination

14.1.2 U:GET (Compressed Data Point Retrieval Subroutine)

$U: G E T$ is a subroutine by which U:TGR and U:WFA retrieve compressed data points from the compressed data buffer (U.CMP). By manipulating the return location, the subroutine identifies the absence of compressed points for the desired channel when the buffer is empty. 
A counter (GETCN--Get Counter) is used to point to the next data point (consisting of three computer words, one of phase and two of BCD time) in the buffer. When the compressed point counter (U.CPS) reaches zero, GETCN is reset to zero to effectively empty the buffer.

The calling sequence is:

$\begin{array}{ll}\text { BLM, } 14 & \text { U:GET } \\ \text { BRU } & \text { NONE }\end{array}$

(DESIRED CHANNEL IS IN R6)

(RETURN LOCATION IF NO COMPRESSED POINTS)

(RETURN WITH PHASE IN R17, BCD TIME IN $\mathrm{R} 12,13)$

\subsection{Called Subroutines}

SUBROUT INE PROGRAM

$E: C O T \quad E \$ D I F$

E:DIF $\quad E \$ D I F$

U:WFA U\$WA

U:RP2 U\$REP
FUNCTION

Converts the $B C D$ time characters to the binary count of seconds.

Used to determine the time difference in seconds between two time values, one in binary and the other in BCD characters.

The program dedicated to the waveform analysis after onset has been identified by U:TGR.

That portion of the Reportable Signal Description message generator which initiates reports for Part II of the RSD to conclude the report message.

\subsection{Data Formats}

\subsection{1 $\underline{\text { TRBUF }}$}

The compressed data points of a potential trigger are stored in a buffer called TRBUF (Trigger Buffer). Each entry requires three 
computer words, the first gives the phase value, and the next two give the BCD characters of the DOY/TOD group. Since the buffer is purged by U:TGR and/or U:WFA each ten seconds, it is designed to hold a maximum of ten entries at any one time, for each channel. This amounts to 30 words per channel for 14 channels, or $42 \emptyset$ words. The ten entries for each channel are arranged in continuous memory locations. A 14-element table called TRBFP (Trigger Buffer Pointer) indicates the first location (of the $3 \emptyset$ ) used by each sensor channel.

\subsection{Input Parameter Descriptions}

PARAMETER PROGRAM DESCRIPTION

U.CPC U\$DAT Channel compressed point counter

U.CPP U\$DAT Pointers to the beginning of each channel's section of U.CMP.

U.AST U\$DAT Channel waveform analysis status, indicates point in signal to which processing has progressed.

U.CDT U\$WF The phase value of a likely candidate in the peak detection algorithm.

U.CDM U\$WFA The DOY/TOD group for a candidate in the peak detection algorithm.

U.AMP U\$DAT Amplitude estimate for current ten second segment.

U.OAM U\$DAT Amplitude value assigned as amplitude at onset.

U.DIU U\$DAT The diurnal slope estimate, an estimate of the long term trends in phase deviation. 
14.4 Input Parameter Descriptions (continued)

PARAMETER PROGRAM DESCRIPTION

U.ODI U\$DAT The diurnat slope value assigned at onset.

U.OFZ U\$DAT Phase value at onset.

U.OTM U\$DAT Time of onset, binary count of seconds for day.

U.OTB U\$DAT Time of onset, BCD characters for DOY/TOD group.

U.CST U\$DAT Channel status, used to indicate validity of input data based on amplitude monitoring program evaluation.

U.OSG U\$DAT The standard deviation of the incoming phase signal assigned to the onset.

U.SIG U\$RTA Computed standard deviation of incoming phase (see Section 12.3.1).

U.DTM U\$DAT Parameter used by E\$DIF to permit finding time difference between any two values (program designed to find difference between onset time and given value).

U.END U\$DAT Bits used to flag conditions under which signal processing was terminated.

U.CLS U\$DAT Flag to indicate which set of criteria was passed; $=1$ for class A criteria, +2 for class B, +3 for COCO signal.

U.LTF U\$DAT The longer time constant factor for the digital filter of the diurnal estimate.

U.FAC U\$DAT The update factor used in the digital filter for the diurnal estimate.

U.LFZ U\$WF The most recent compressed data point for each channel.

U.LTM U\$WFA The DOY/TOD group for U.LFZ for each channel. 
14.5 Output Parameter Descriptions

PARAMETER SIZE DESCRIPTION

U.TRF $\quad 14 \quad$ The status flag of processing of triggering sequence (see Section 14.1.1).

U.TRL $14 \quad$ The count of compressed points required to trigger waveform analysis (see Section 14.1.1).

U.TGR Entry point for onset detection program.

14.6 Internal Data Descriptions

PARAMETER SIZE DESCRIPTION

TRCNT $\quad 14 \quad$ Count of points currently under consideration for potential onset.

TRBUF $\quad 420 \quad$ Buffer for compressed data points in possible triggering sequence. Three words per data point $\times 14$ channe $75 \times 10$ points per channe 1 .

TRBFP

14

Pointer to first. word of each sensor channel's partition of TRBUF.

PONST

28

Binary value for possible onset time.

TEMP

1

Working storage.

TEMP 1

1

Working storage.

TRTIM

$=3010$; maximum number of seconds permitted between first and last points of triggering sequence.

GETCN

Counters used to point to next data point in compressed data point buffers. 


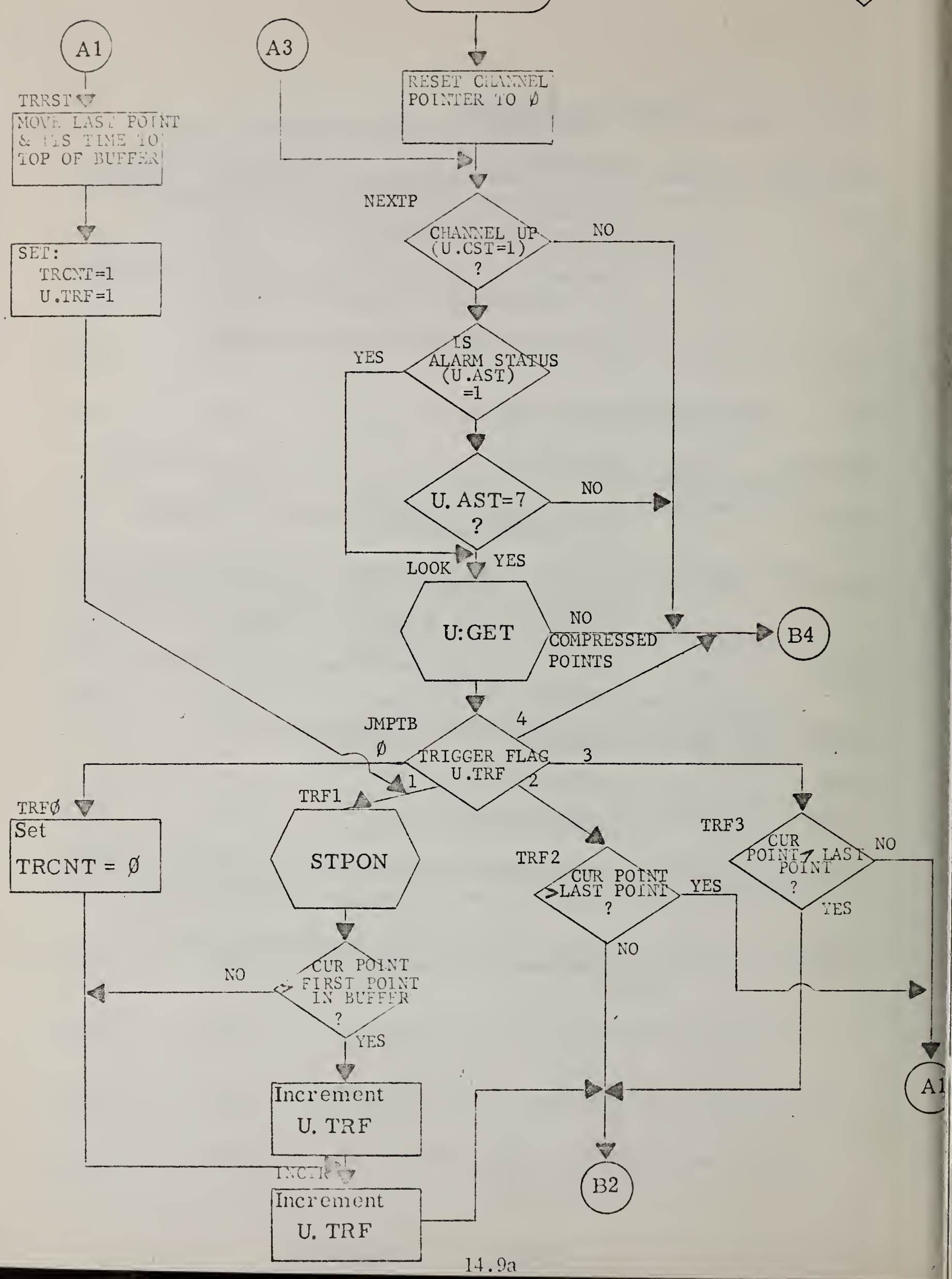




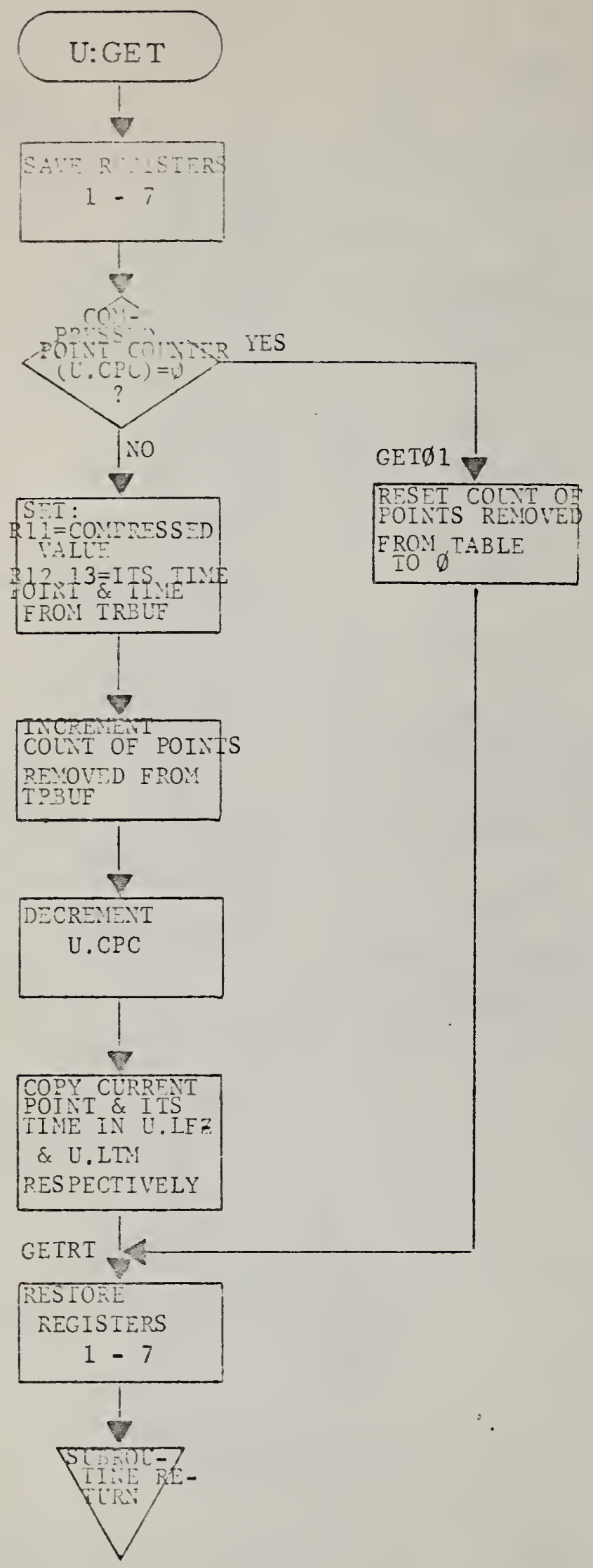

\section{STPON}

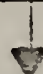

STCRE BCD

II YiE FRO:I RI2,

RI 3 FCR LAIER

BOCONEL AND

PARUEIER

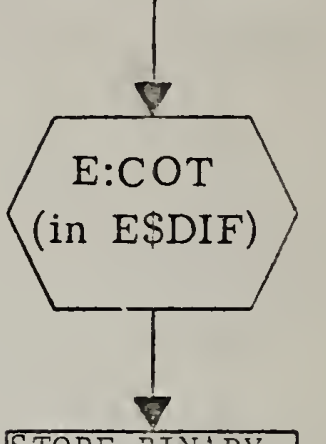

STORE BINARY EQUIVALENT OF BCD ONSET TIME IN PONST

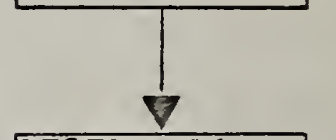

RES TORE R12, R13 TO BCD

VALUE

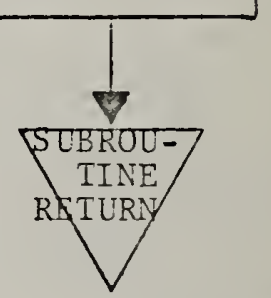


15. U\$WFA

15.1 Functional Description

The last program entered (after U:RTA, U:LSQ, and U:TGR) at the end of each ten-second interval is U:WFA ( $\underline{U}$ Signal Waveform Analysis). Whereas U:TGR is responsible for detecting signal onsets, U:WFA handles the remainder of the waveform analysis. This consists of progressing through the waveform, recognizing key points, determining whether the characteristics for the section just completed meet predetermined criteria, and permitting the processing to continue if they do. The primary vehicle for determining the point in the waveform, and which section of U:WFA to execute, is a flag called U.AST (U Signal Alarm Status). Figures 15.1, 15.2, and 15.3 wi11 illustrate the values U.AST will take during the various sections of the three waveforms which NUSTAD will recognize.

Upon entry, some status checking is done, and waveform processing is performed for those channels which are not down. U.AST is examined, and control is transferred to that portion of the program responsible for the current section of the waveform.

When U.AST $=1$, there is no signal currently in progress. The diurnat slope estimate (U.DIU) is updated (see Section 15.3.1) and processing is considered complete for that channel. When all channels are done, control is returned to the scheduler. 


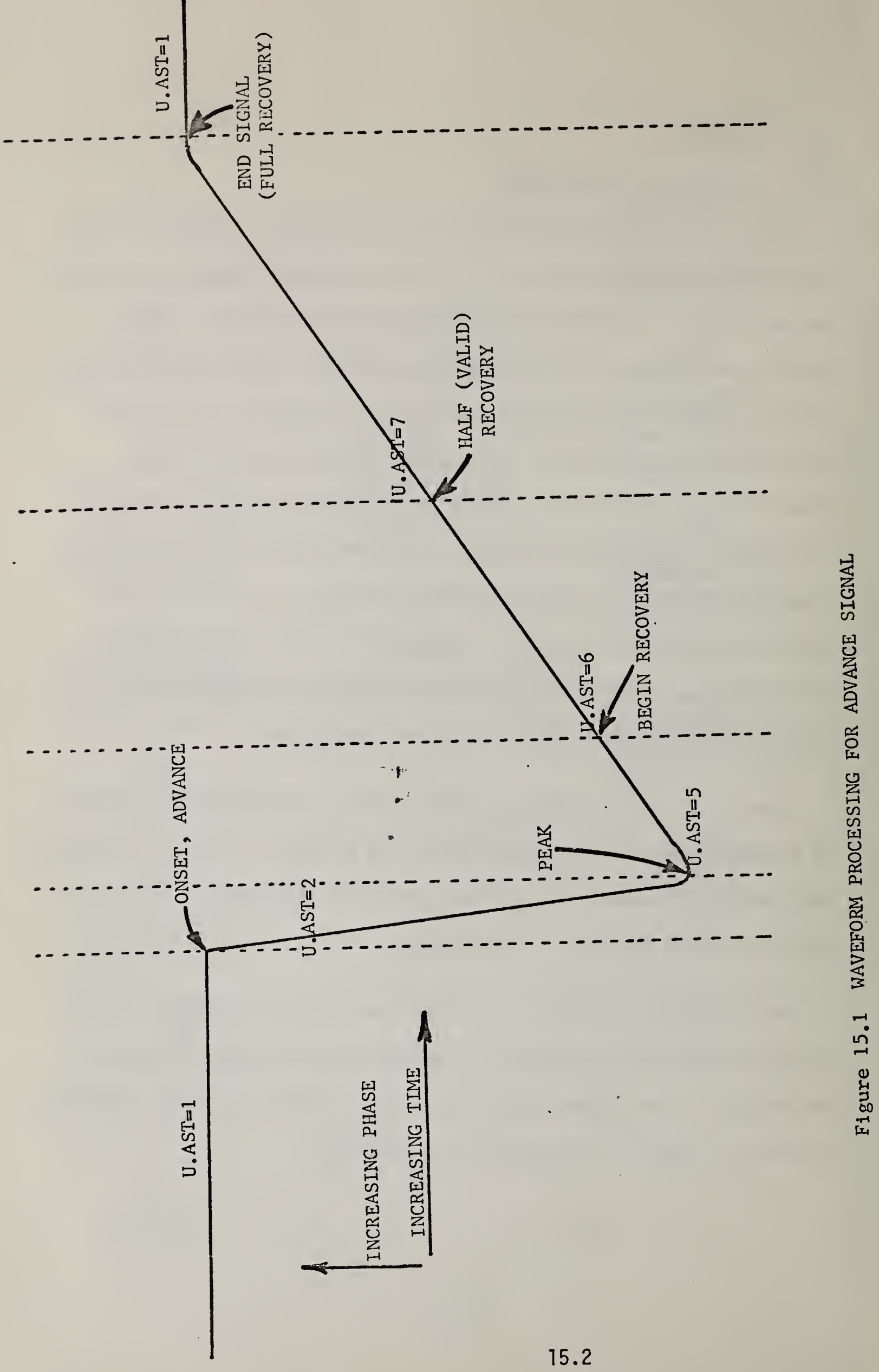




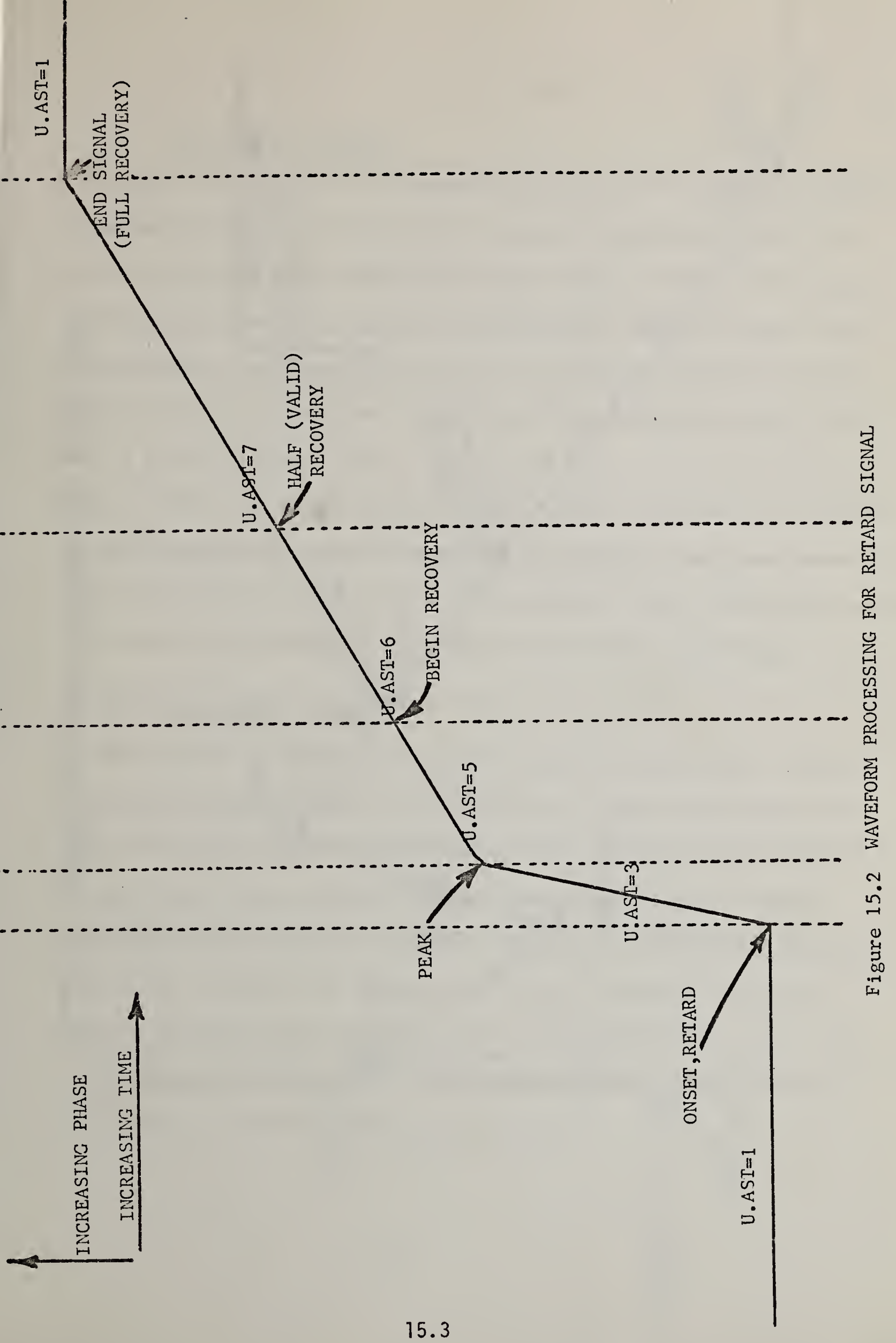




\subsubsection{Peak Search, Advance}

When U.AST $=2, U: T G R$ has detected an onset in the advance direction (decreasing numeric values) and the section of U:WFA called UAST2 will seek the signal's peak. The minimal phase value is considered the peak, so the search consists of merely determining the smallest numeric value. The smallest value encountered is held in U.CDT, the candidate for the position of signal peak, until four consecutive compressed points follow with a greater numeric value to confirm the point. If no point meeting these restrictions is encountered with $\mathrm{Tl}$ seconds (currently established as ten minutes), the signal is rejected and U.AST is reset to 1 to again try to determine a signal onset. When a possible peak is confirmed, control is passed to the section of the program called PVALC (see below).

\subsubsection{Peak Search, Retard}

When U.AST $=3$, U:TGR has detected an onset in the retard direction (increasing numeric values) and the section of U:WFA called UAST3 will seek this signal's peak. The inflection point where the slope of the retard signal reduces significantly is considered the peak. NUSTAD computes the slope between the onset point and each succeeding compressed data point, and seeks the maximum such value. When four consecutive onset to current point slopes fall under $75 \%$ of the maximum slope, the peak has been confirmed. As in UAST2, the TI second limit is enforced and control is passed to PVALC when successfut. 


\subsubsection{Peak Validity Check}

PVALC is tasked to determine whether the onset characteristics between the now identified onset and peak meet the predetermined criteria. The pha offset, compensating for the long term trend at onset, and the average rate of onset are computed (see Section 15.3.2). These values are applied against the following standards, and U.CLS is set accordingly:

U.CLS $=1$ if:

Onset rate (W.ONR) not less than one of the following values, based on the tracking rate (U.TRK):

PONLO ( 6 microseconds/minute) for tracking rates $\emptyset, 1$, or 2 . PONMD ( 76 microseconds/minute) for tracking rates 3,4 , or 5 . PONHI (20 microseconds/minute) for tracking rates $6,7,8$, or 9.

And the phase offset was not less than 10 microseconds.

\section{U.CLS $=2$ if:}

Signal was in the advance direction.

And the onset rate was not less than 3 microseconds/minute.

And the phase offset was not less than 2 microseconds.

If the computed onset rate (W.ONR) and phase offset (W.OST) do not meet these cirteria, the signal is rejected as being impulsive noise. If either of the sets of criteria are passed, U.AST is set to 5 and processing continues.

\subsubsection{Begin Recovery}

When U.AST $=5$, a valid peak has been encountered, and control is passes: to the section of U:WFA called UAST5 to seek out the beginning of the phase recovery portion of the waveform. At peak, the long term trend estimate (U.DIU) was reset to the onset value (U.ODI). Recovery begins 
when the phase begins a trend in the retard direction. This is identified when U.DIU, operating with U.FAC $=.10$, exceeds the onset value (U.ODI) by ALPHA (currently set to .3 mircoseconds/minute). If this occurs within T2 seconds (currently set at $2 \emptyset$ minutes) after onset, the beginning of recovery is confirmed and U.AST is set to six. If not, it's another reason to reject the signal.

\subsubsection{Valid Recovery}

When U.AST $=6$, recovery has begun. The next key point in the waveform is the half, also known as valid, recovery point. This is reached when the following conditions have been met:

a. The phase has recovered within the following limits:

(1) ADVANCE SIGNAL, to within half of the difference between onset and peak (Figure 15.1).

(2) RETARD SIGNAL, to within half of the difference between peak and one cycle above onset (Figure 15.2).

(3) MULTIPLE PEAK ADVANCE SIGNAL, to at least three microseconds from the final peak value (Figure 15.3).

b. Ten minutes (T3, the minimum time for full slope recovery) has elapsed since onset without the current long term trend (U.DIU) recovering to within BETA (currently .2 microseconds/minute) of the long term trend estimate at onset (U.ODI).

Al1 the while we are attempting to process this part of the waveform, the possibility of a multiple peak signal exists. Therefore, the maximum 
phase value and $i$ ts time are saved. In the event an advance signal fails to meet point $b$, above, U.AST is reset to two to attempt to find another peak while the signal class (U.CLS) and direction (U.ODR) are set to 3 to indicate a multiple peak advance signal. When UAST2 finds another peak, the previous reporting criteria are ignored and U.AST will again be set to five. If any other type signal fails point $b$, above, the signal is rejected. When the above points are satisfied, U.AST is set to seven to search for the end of the signal. At this time, the first part of the report generator is called (U:RP1) to report on the waveform between onset and the valid recovery point (see Section 17.1, U:REP).

\subsubsection{Terminate Recovery}

When U.AST $=7$, the signal is considered valid and the first part of the report message (called the RSD, Reportable Signal Description) is output to the logging teleprinter via U:REP. The final task of the analysis program is to termine processing on the current signal, which may be done in one of several ways:

a. NORMAL TERMINATION. When the phase recovers to within TREC (currently set at three) microseconds of the onset value AND the long term trend estimate (U.DIU) recovers to within BETA (.2 microseconds/minute) of the long term trend estimate at onset (U.ODI). 
b. TIME LAPSED TERMINATION. When T4 seconds (currently set at one hour) passes since onset.

c. SECOND ONSET DISCOVERED. While U.AST $=7$, U:TGR wi11 be permitted to look for new signals which may occur on the tail end of the current signal. During this period, though, the number of points required to trigger a new signal (U.TRL) is set to eight, requiring more certainty in calling a phase excursion a probable signai, and terminating the processing of the current signal.

d. SIGNAL LOST. If the channel should go down while U.AST is either 6 or 7 , the chance that a reportable signal was in progress is sufficient to warrant generating a RSD with the notation that the signal was terminated because of signal drop out.

\subsubsection{Signal Rejection}

When a signal is rejected for any reason, a flag called REASN is set to a value corresponding to the reason, and a part of U:WFA called FAIL is entered to reset flags and variables to permit NUSTAD to begin looking for a new signal on that sensor channel. If the operator has switch register bit $1 \emptyset$ set, the failure will be indicated for the operator on the ASR 35 teleprinter, under the following format: 


\section{$\underline{\mathrm{DDD}} \underline{\mathrm{TTTTT}} \underline{\mathrm{C}} \underline{\mathrm{R}}$}

Where $\quad$ DDD is a three character DOY

TTTTTT is a six character TOD

$\underline{C}$ is the sensor channel indicator ( $A$ thru $N$ )

$R$ indicates a rejected signal

$\underline{n}$ is the reason, interupted as follows:

1 Excessive time, onset to peak.

2 Retard signal fails offset criteria.

3 Advance signal fails secondary offset criteria.

4 Advance signal fails secondary onset rate criteria.

5 Excessive time, onset to beginning recovery.

6 Slope recovery too soon after onset.

7 Recovery rate greater than onset rate.

Table 15.7

Signat Rejection Message Format

After each section completes what processing it can with the

data available, the diurnal slope estimate is up dated for that channel

and U:WFA goes on to the next sensor channel.

When U:WFA completes all processing, control is returned to the operating system at the scheduler reentry point (E:SKD). 
15.2 Called Subroutines

SUBROUTINE PROGRAM FUNCTION

U:RP1 U\$REP Generates Part I of RSD at valid signal point in waveform.

U:RP2 U\$REP Generates Part II of RSD at final recovery point in waveform.

U:GET R\$TGR Routine to retrieve compressed data from the compressed data point buffer.

E:DIF E\$DIF Computes the time difference between onset and later point in time.

E:SKD E\$SKD Operating system scheduler entry point.

E:COT E\$DIF Converts the BCD time characters to the binary count of seconds.

E:035 E\$T35 Transfers output message to buffer before transmission to the ASR 35.

E:FRT E\$FRT Reads current BCD time and reformats for output message.

\subsection{Computational Methods}

15.3.1 Digital Filter in Diurnal Estimate Algorithm

The least squares slope estimate described in Section 13.3 is used to determine the short term ( $19 \emptyset$ second) trends of the input phase samples. These short term estimates are put through a long time constant first order digital filter to provide the long term trend estimate which NUSTAD labels the diurnal slope (U.DIU). 
The first order digital filter performs the arithmetic manipulation

$$
y_{(n T)}=(1-K) y(n T-T)=K x(n T)
$$

Where

$$
\begin{array}{ll}
{ }^{y}(n T) & \text { is the filter output at time } t=n T \\
{ }^{y}(n T-T) & \text { is the previous filter output } \\
{ }^{x}(n T) & \text { is the filter input at time } t=n T \\
K & \text { is the constant with limits such that } \emptyset<K<1 \\
T & \text { is the time between samples }
\end{array}
$$

The filter is depicted in Figure 15.4, with $\mathrm{Z}^{-1}$ denoting the $T$ second delay.

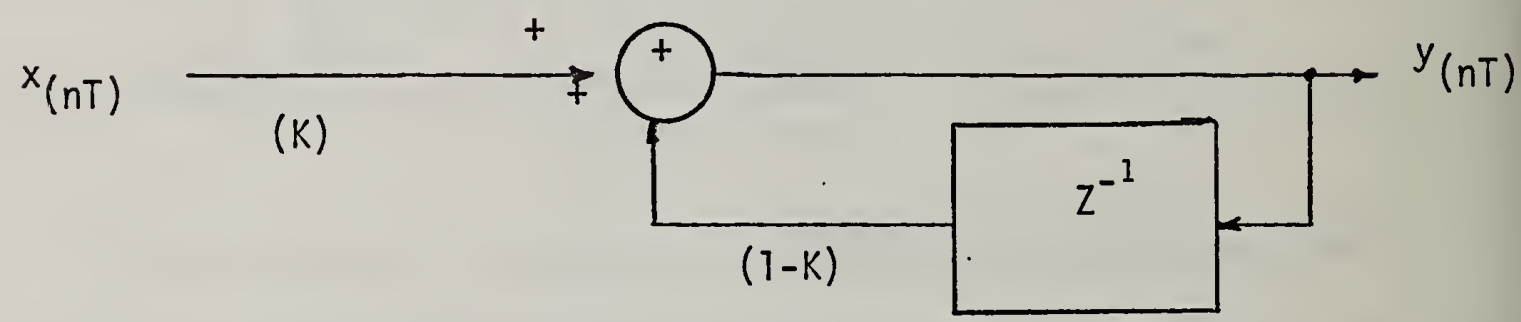

Figure 15.4 First Order Digital Filter

Equation (1) is rewritten to reduce the number of multiplication operations required during real time execution:

$$
y_{(n T)}=y_{(n T-T)}=k\left(x_{(n T)}-y_{(n T-T)}\right)
$$


The characteristics of the first order digital filter will be considered by examining the filter's response to step and impulsive inputs. For the step input, consider the following conditions applied to equation (2):

$$
\begin{aligned}
& y(\emptyset)=\emptyset \\
& x_{(n T)}= \begin{cases}\emptyset & n=\emptyset \\
1 \emptyset & n \neq \emptyset\end{cases}
\end{aligned}
$$

Figure 15.5 displays the response over ten minutes ( 690 seconds) for the two values used by NUSTAD $(K=.95$ and $K=.10)$ as well as two greater values to illustrate the relationship of $K$ to the response time. In all cases $y_{(n T)}$ approaches $x_{(n T)}=10$ as $n$ increases, but does it slower for smaller $k$.

The conditions imposed for the impulsive response are:

$$
\begin{aligned}
& y_{(\emptyset)}=\emptyset \\
& x_{(n T)}= \begin{cases}1 \emptyset & n=2 \emptyset \\
\emptyset & n \neq 2 \emptyset\end{cases}
\end{aligned}
$$

Figure 15.6 illustrates the response of the first order digital filter to an impulse and plots its response over a period of 5 minutes. Note that the effect of the impulse is lessened by smaller values of $\mathrm{K}$; i.e., the maximum offset is directly proportionai to $\mathrm{K}$. However, the decay time increases with decreasing $K$. Also note the similarity between the impulse and step responses shown in Figures 15.5 and 15.6 and those of a first order analog filter (low pass RC filter). 


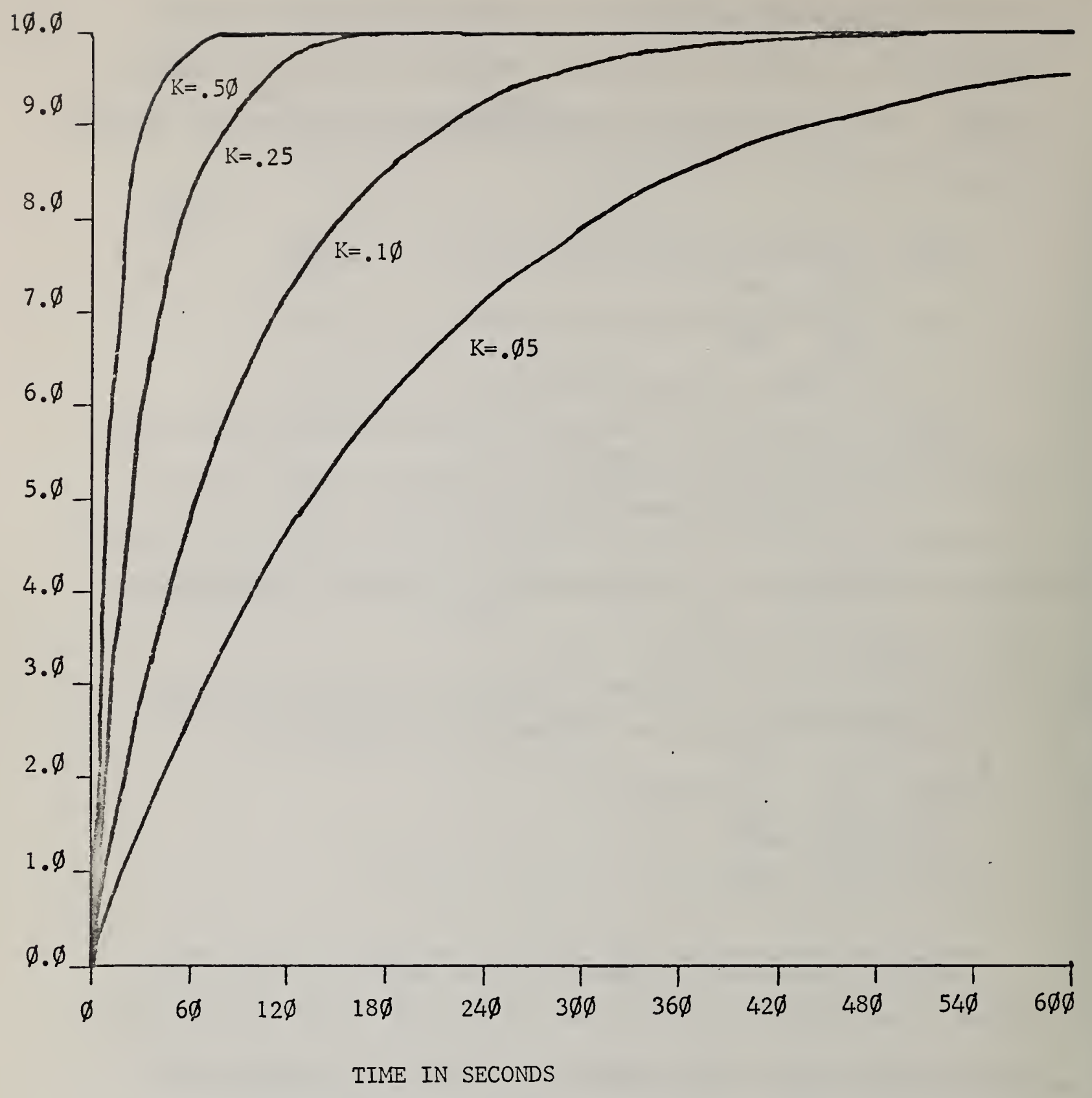

Figure 15.5 Step Response of First Order Digital Filter 


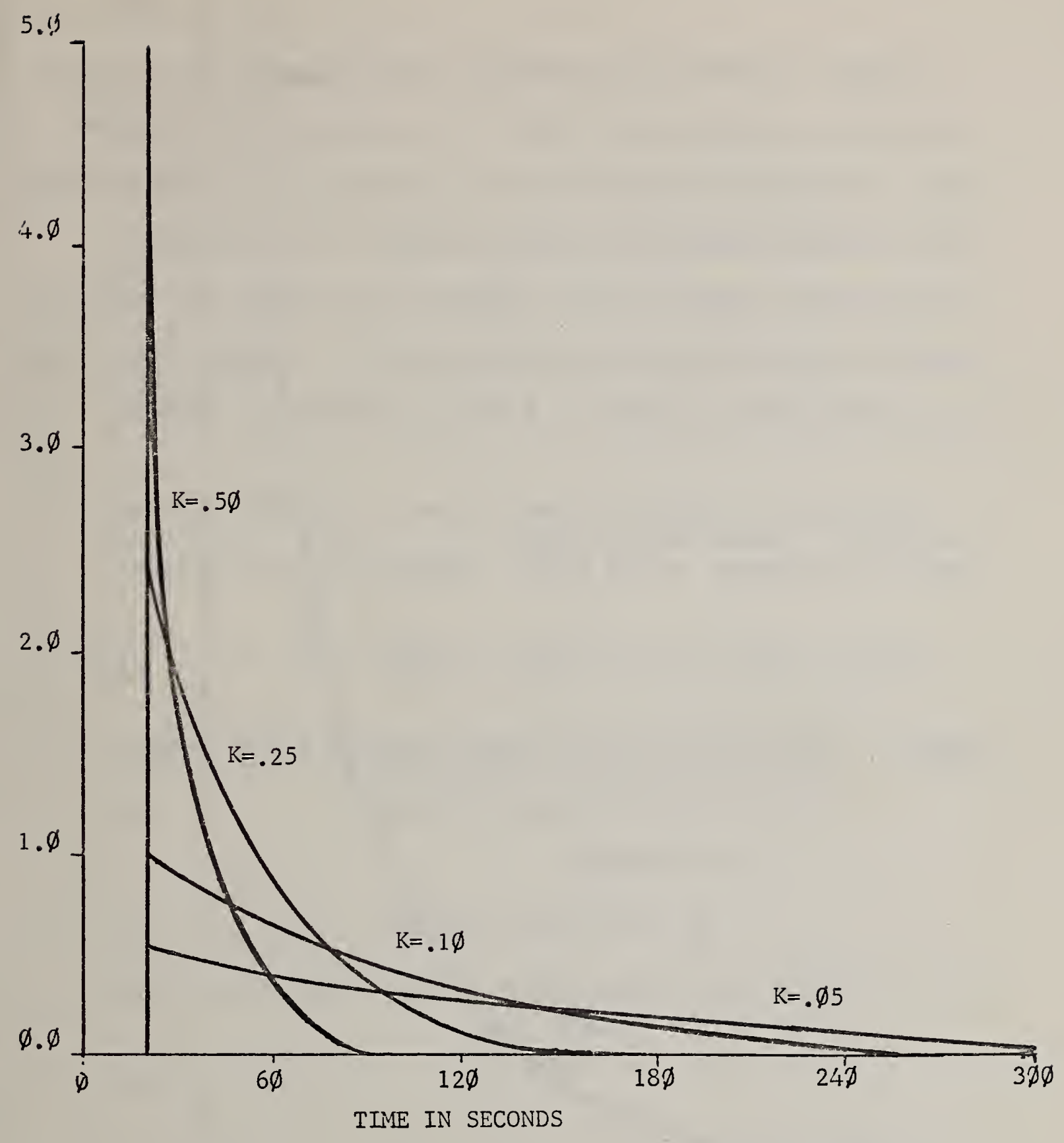

Figure 15.6 Impulse Response of First Order Digital Filter 
Engineers unfamiliar with digital filters of this type generally request data concerning the filter's time constant (TC). For the sake of comparison to analog devices, Figure 15.7 is included to show the relationship between the values assigned $K$ and a computed equivalent time constant (TC). The curve was arrived at by plotting the time at which $83 \%$ of the maximum offset of a stepped input signal was reached vers us the value of $K$ used in equation (2) above.

In NUSTAD, the digital filter is used to determine the long term trend estimate, called U.DIU. Equation (2) then becomes:

$$
U . D I U=U . D I U+U . F A C *(U . S L P-U . D I U)
$$

where: U.DIU is the diurnal slope (long term trend) estimate U.FAC is the filter update factor:

$=.95$ normally

$=.10$ while seeking recovery

U.SLP is the least squares slope estimate from U:LSQ. 


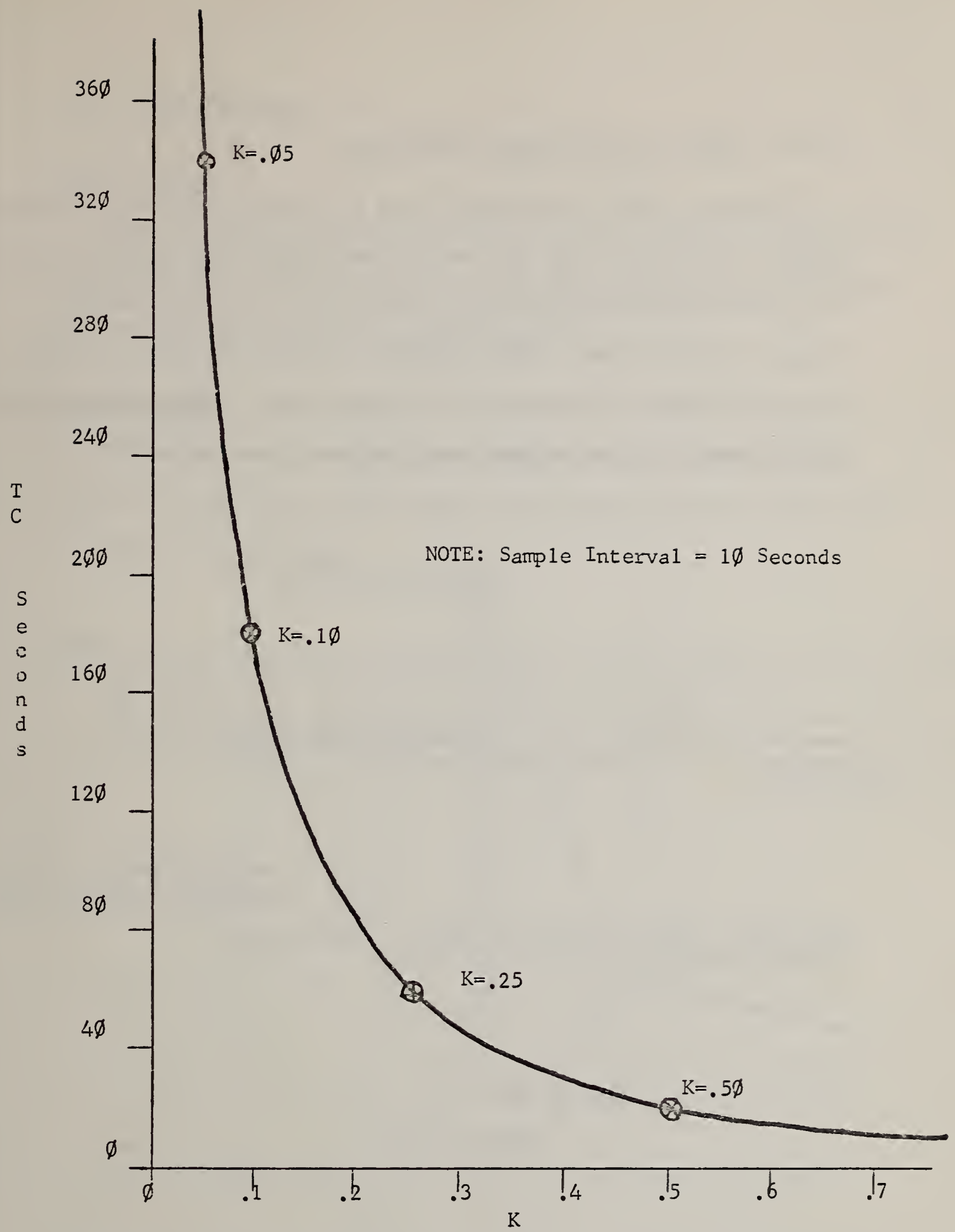

Figure 15.7 Relationship Between $\mathrm{K}$ and Filter Time Constant (TC) 


\subsubsection{Offset and Onset Rate Computation}

The phase offset between onset and peak consists of the difference between the phase values at onset and peak, minus the product of the long term trend estimate at onset multiplied by the time interval between onset and peak. This attempts to cancel the effect of the long term trends in determining the phase offset. That value divided by the amount of time between onset and peak gives the onset rate.

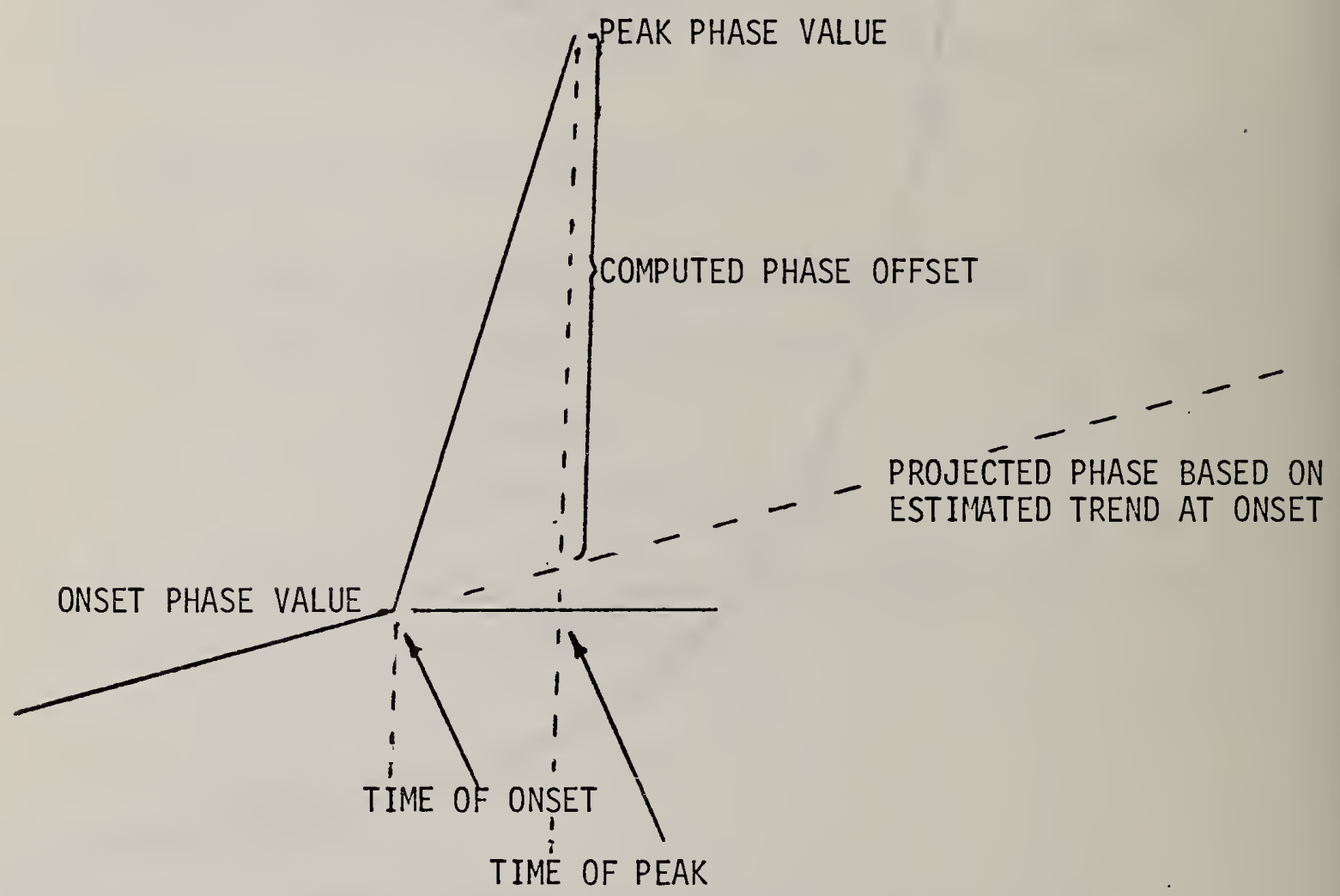




\subsection{Data Formats}

15.4 .1 U.DIU

Since the long term slope estimates are the result of filtering the short term slope estimates (U.SLP), the data format will be the same. See Section 13.4.3 for the format of U.SLP.

\subsubsection{U.ONR}

Unlike the slope representation used for U.SLP and U.DIU (see Section 13.4.3), the average onset rate between onset and peak (W.ONR) uses the following format:

BITS \begin{tabular}{|l|l|l|l|l|l|l|l|l|l|l|l|l|l|l|l|}
\hline & 1 & 2 & 3 & 4 & 5 & 6 & 7 & 8 & 9 & 10 & 11 & 12 & 13 & 14 & 15 \\
\hline
\end{tabular}

Figure 15.9 Format of Computed Onset Rate (W.ONR) 
15.5 Input Parameter Descriptions

PARAMETER PROGRAM DESCRIPTION

$\begin{array}{ll}\text { U.CST U\$DAT } & \text { Channel status, used to indicate validity } \\ \text { input data based on amplitude monitoring }\end{array}$ program evaluation.

U.AST U\$DAT Channel waveform analysis status, indicates point in signal to which processing has progressed.

U.SLP U\$DAT Computed slope estimate in microseconds per minute. Indicates short term data trends.

U.DIU U\$DAT The diurnal slope estimate, an estimate of the long term trends in phase deviation.

U.FAC U\$DAT The update factor used in the digital filter for the diurnal estimate.

U.CTM U\$DAT Two words of BCD time for the last samples read; i.e., the current time for the ten-second segment.

U.CLS U\$DAT Flag to indicate which set of criteria was passed: $=1$ for class $A$ criteria, $=2$ for class $B,=3$ for COCO signal.

W.PFZ U\$DAT Phase value at signal peak.

W.PTM U\$DAT BCD time of peak value.

W.PAM U\$DAT Signal amplitude at peak.

W.OST U\$DAT Computed phase offset, onset to peak (see Section 15.3.2)

W.ONR U\$DAT Average onset rate, onset to peak.

W.LPK U\$DAT Phase value of first peak of multiple peak advance signal.

W.LTM U\$DAT Time of first peak of multiple peak advance signal.

W.VPK U\$DAT Phase value of valley peak of multiple peak advance signal. 


\subsection{Input Parameter Descriptions (continueci)}

\section{PARAMETER PROGRAM DESCRIPTION}

$\begin{array}{ll}\text { W.VTM U\$DAT } & \text { Time of valley peak of multiple peak advance } \\ \text { signal. }\end{array}$

W.RFZ U\$DAT Phase value at valid recovery point.

W.RTM U\$DAT Time of valid recovery point.

W.RAM U\$DAT Signal amplitude at valid recovery point.

U.END U\$DAT Bits used to flag conditions under which signal processing was termiriated.

U.OFZ U\$DAT Phase value at onset.

U.0DI U\$DAT Long term data trend estimate (U.DIU) at onset.

U.BGF U\$DAT The shorter time constant factor for the digital filter of the diurnal estimate.

U.LTF U\$DAT The longer time constant factor for the digital filter of the diurnal estimate.

U.TRK U\$DAT

Current tracking rate settings for the channels.

U.ODR U\$DAT

Direction of onset, $=1$ for advance, $=2$ for retard, $=3$ after second peak of multiple peak advarice signal.

U.OTM U\$DAT Time of onset, binary count of seconds.

E.IOF E\$T35 Parameter word used in calling E:035.

U.AMP U\$DAT Amplitude estimate for current ten-second segment.

U.DTM U\$DAT Parameter used by E\$DIF to permit finding time differences between any two values (program designed to find difference between onset time and given value).

W.LAM U\$DAT Signal amplitude at first peak of multiple peak advance signal.

W.VAM U\$DAT

Signal amplitude at valley peak of multiple peak advance signal. 


\subsection{Input Parameter Descriptions (continued)}

PARAMETER

U.FRQ

U.TRF

U.TRL
PROGRAM

$E \$ L O G$

U\$TGR

U\$TGR
DESCRIPTION

Current sensor frequency for each sensor channe1.

Status flag of processing of triggering sequence.

Count of compressed points requied to trigger waveform analysis.

15.6 Output Parameter Descriptions

PARAMETER

U:WFA

U.LFZ

U.LTM

U.CDT

U.CDM

28

U.CCL
DESCRIPTION

Entry point for waveform analys is program.

The phase value of the last compressed data point encountered on each channel.

The $B C D$ character time of the last compressed data point encountered on each channel.

The "candidate" peak phase value used in searching for waveform peaks.

The BCD character time of the "candidate" peak points.

Label used to externally reference CYCLE, the number of microseconds in one cycle for the frequencies of each channel.

\subsection{Internal Data Descriptions}

PARAMETER SIZE

SLOPE

14

CFIRM
DESCRIPT ION

S1ope value computed in section UAST3 to determine the inflection point of a retard signal in recognizing signal peak.

Counter used by peak detection routines to confirm the recognition of a signal peak. 
15.6 Interna? Data Descriptions (continued)

PARAMETER SIZE DESCRIPTION

TEMP

TEMP2

T1

T2

T3

T4

POFF

PONHI

PONMD

PONLO

SOFF

SONRT

LOPEK
2

1

1

1

1

1

$=16 \emptyset_{1 \emptyset} ;$ (10 microseconds) Primary offset criteria

$=14 \emptyset_{16} ;(2 \emptyset$ microseconds/minute) Primary onset rate criteria for high tracking rates; i.e., U. $T R K=6,7,8$, or 9

$=\emptyset A \emptyset_{16} ;(1 \emptyset$ microseconds/minute) Primary onset rate criteria for medium tracking rates; i.e., U.TRK $=3,4$, or 5

$=\varnothing 6 \emptyset_{16} ;(6$ microseconds $/$ minute) Primary onset rate criteria for low tracking rates; i.e., U.TRK $=\emptyset, 1$, or 2

$=321 \emptyset$; ( 2 microseconds) Secondary offset criteria $=\emptyset 3 \emptyset_{16} ;(3 \mathrm{microseconds} /$ minute) Secondary onset rate criteria, all tracking rates.

$=89_{10} ;(5$ microseconds) Minimum allowed offset for first peak in multiple peak advance signal. 


\subsection{Internal Data Descriptions (continued)}

PARAMETER

TREC

ALPHA

BETA

RCMIN

CYCLE

REASN

FLMSG
SIZE

1

10

approach onset phase, or next cycle for retard signals, for recovery to be considered complete.

$=38_{1 \emptyset} ;(.3$ microseconds $/$ minute) Point to which U.DIU must exceed U.ODI for beginning of recovery.

$=26_{1 g} ;(.2$ microseconds/minute) Point to which U.DIU must return toward U.ODI for slope recovery to be considered complete.

Microseconds from peak which signal must recover before recovery is considered valid. (See Section 15.1.5.)

14 Number of microseconds in one cycle at the frequency to which each sensor is tuned.

$14 \quad$ Flag indicating why signal was rejected (see Section 15.1.7).

10

"123 123456 C R $R_{2}$; signal rejection message. 


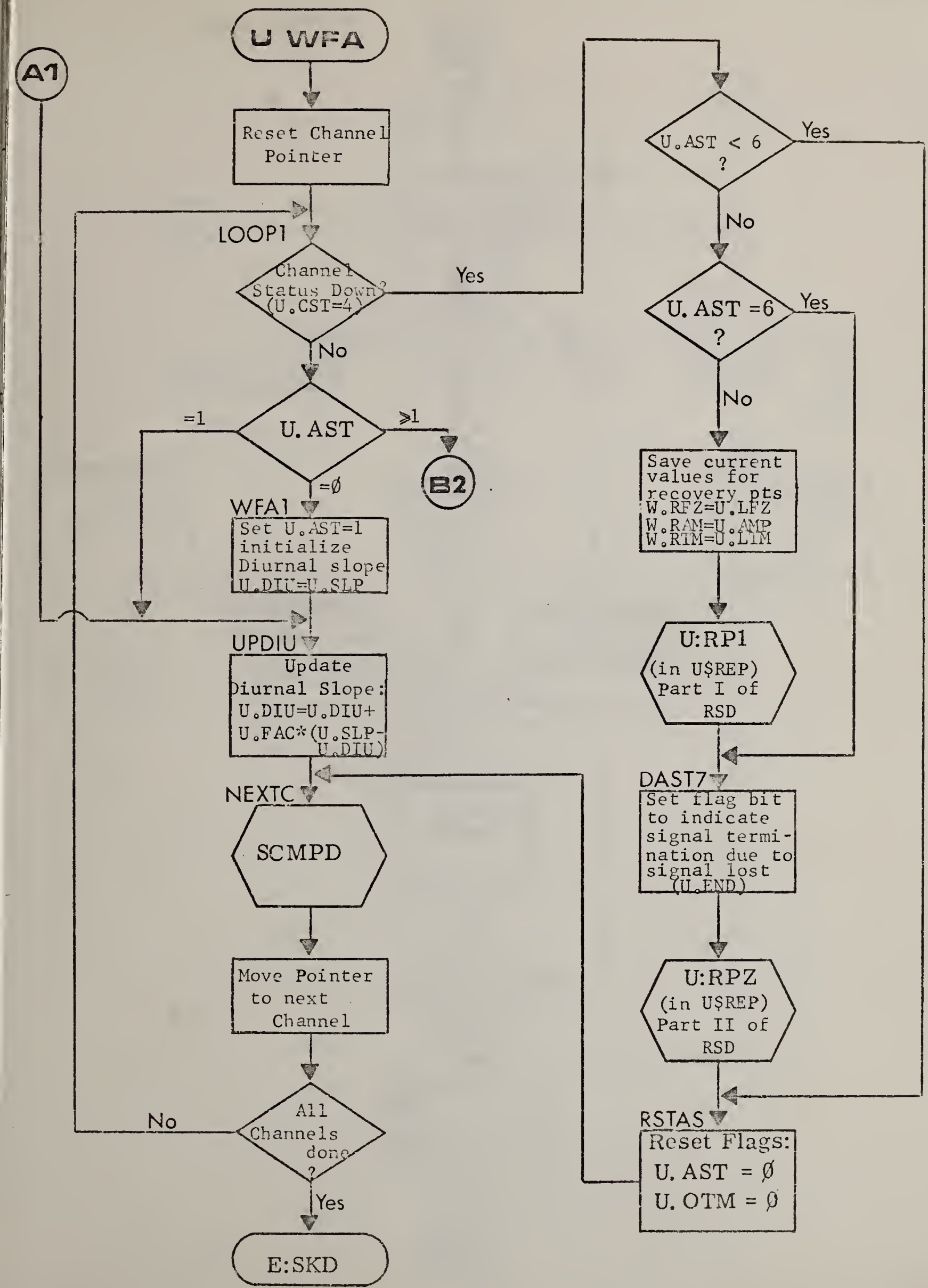




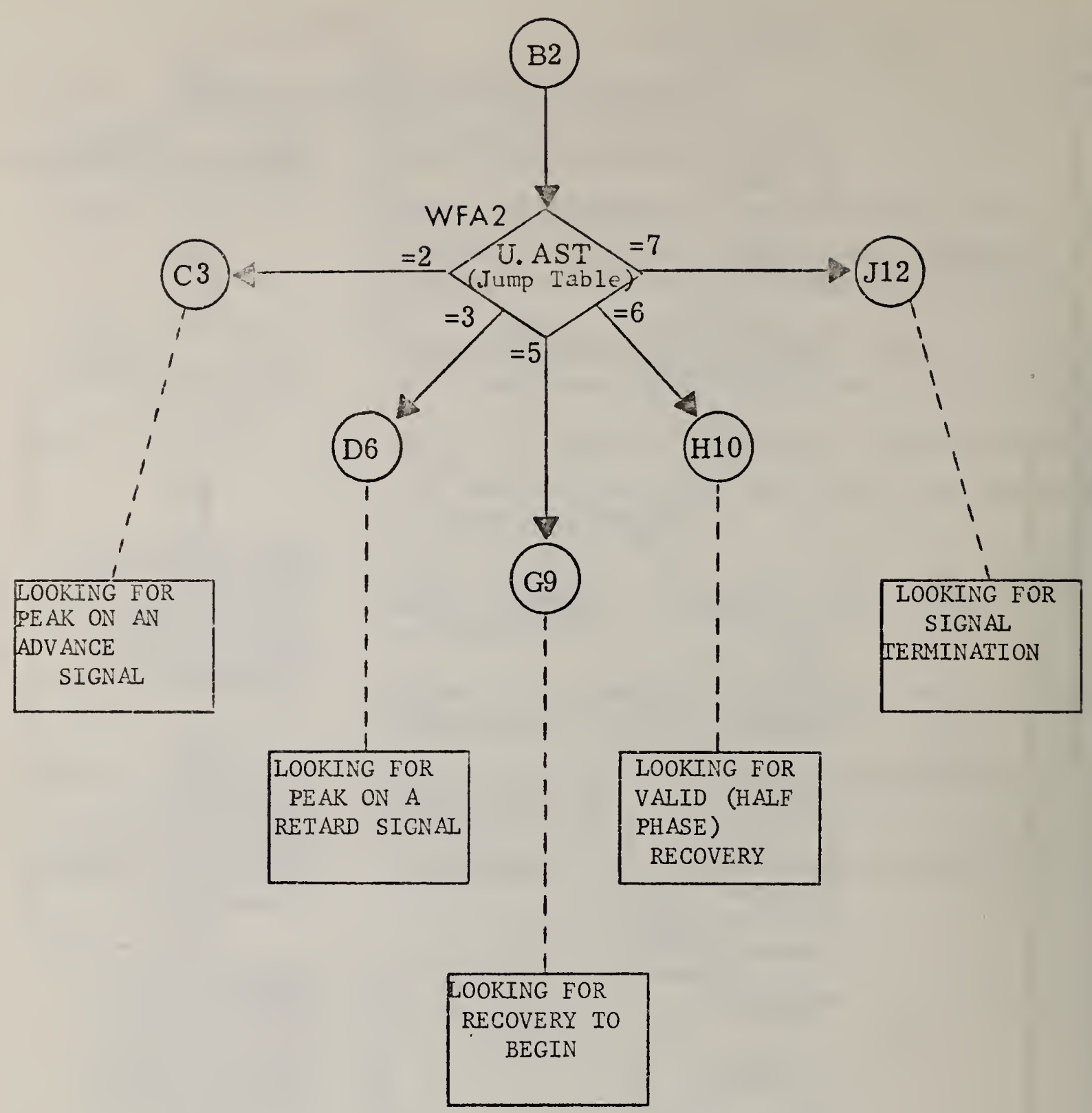




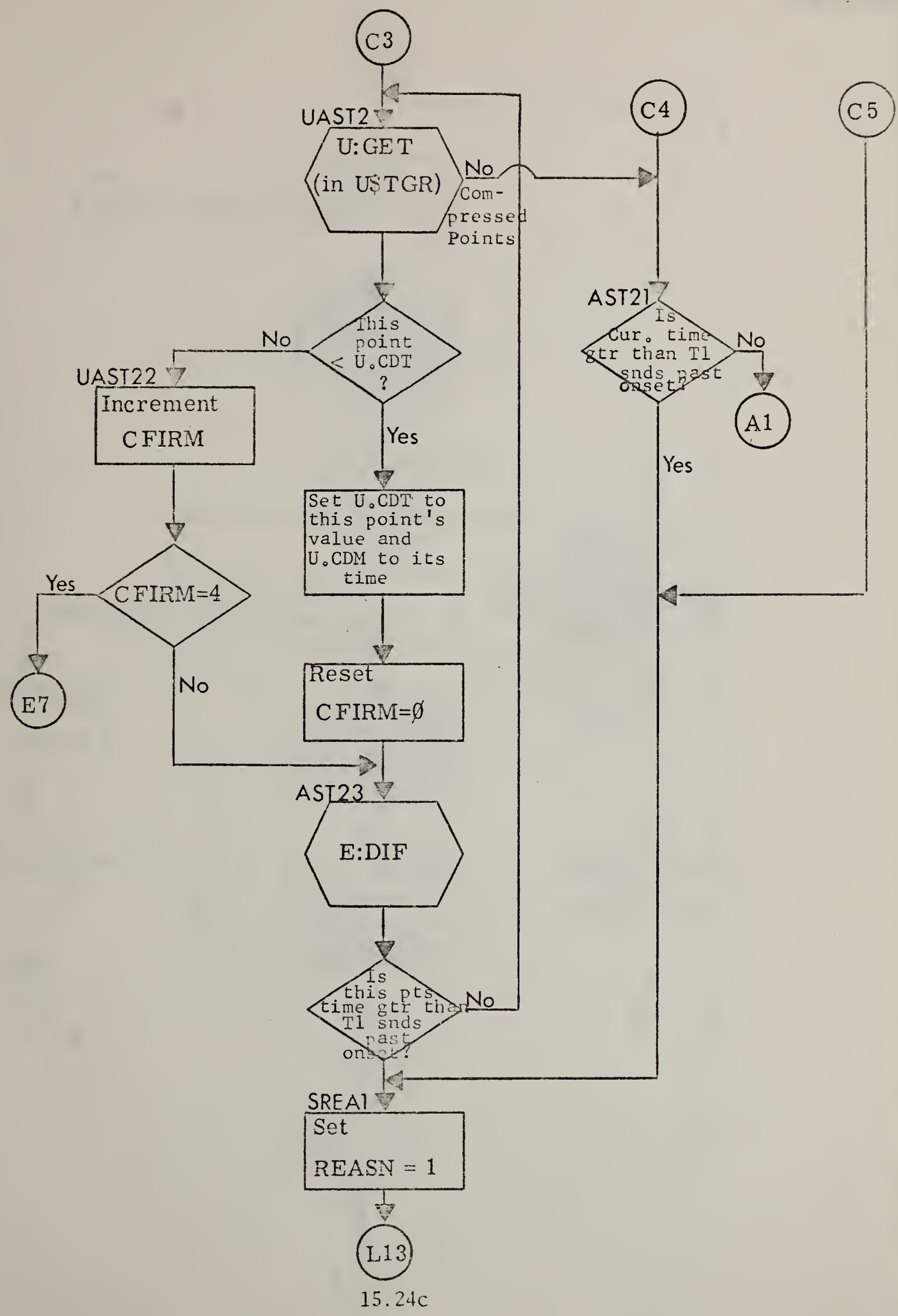




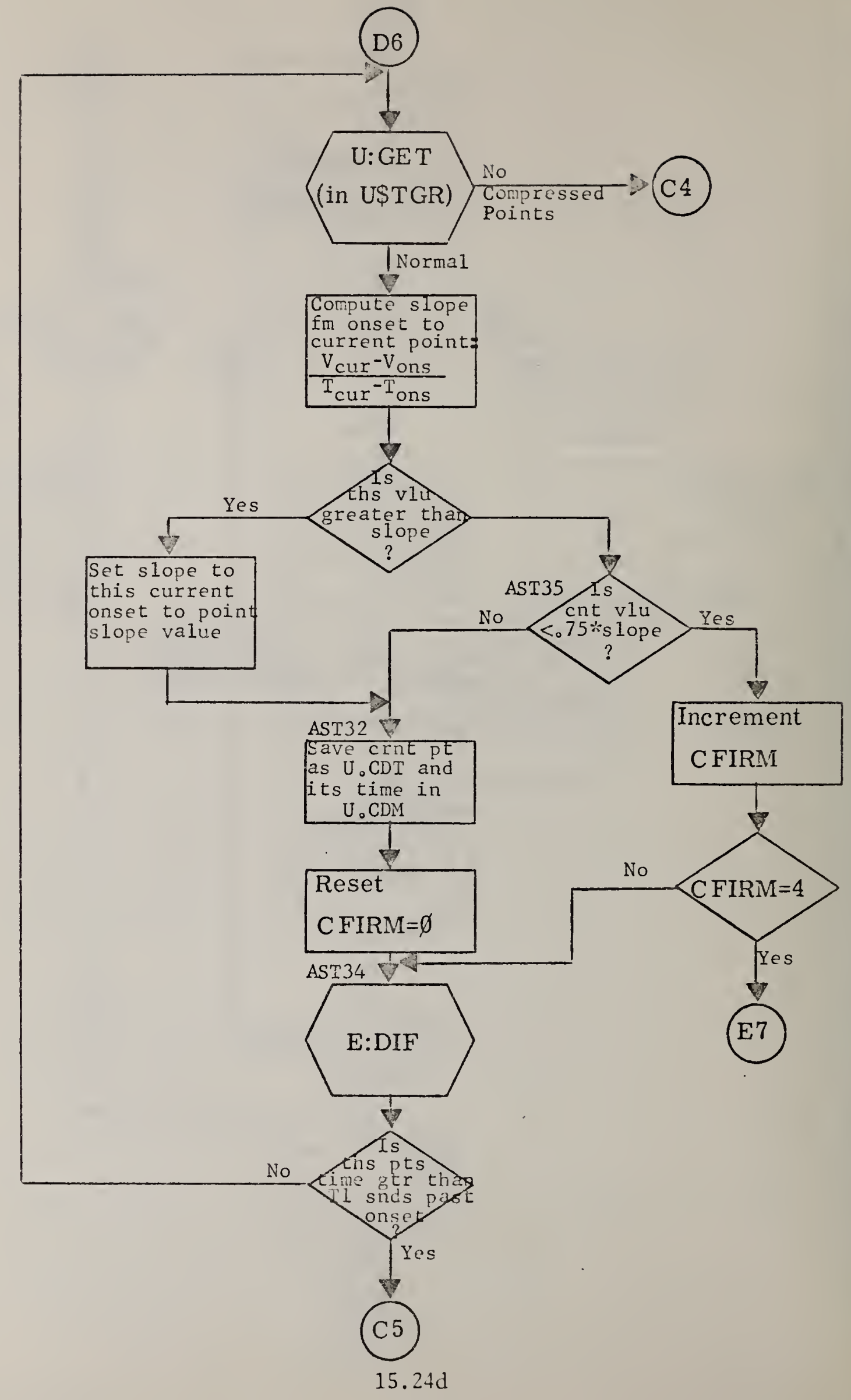




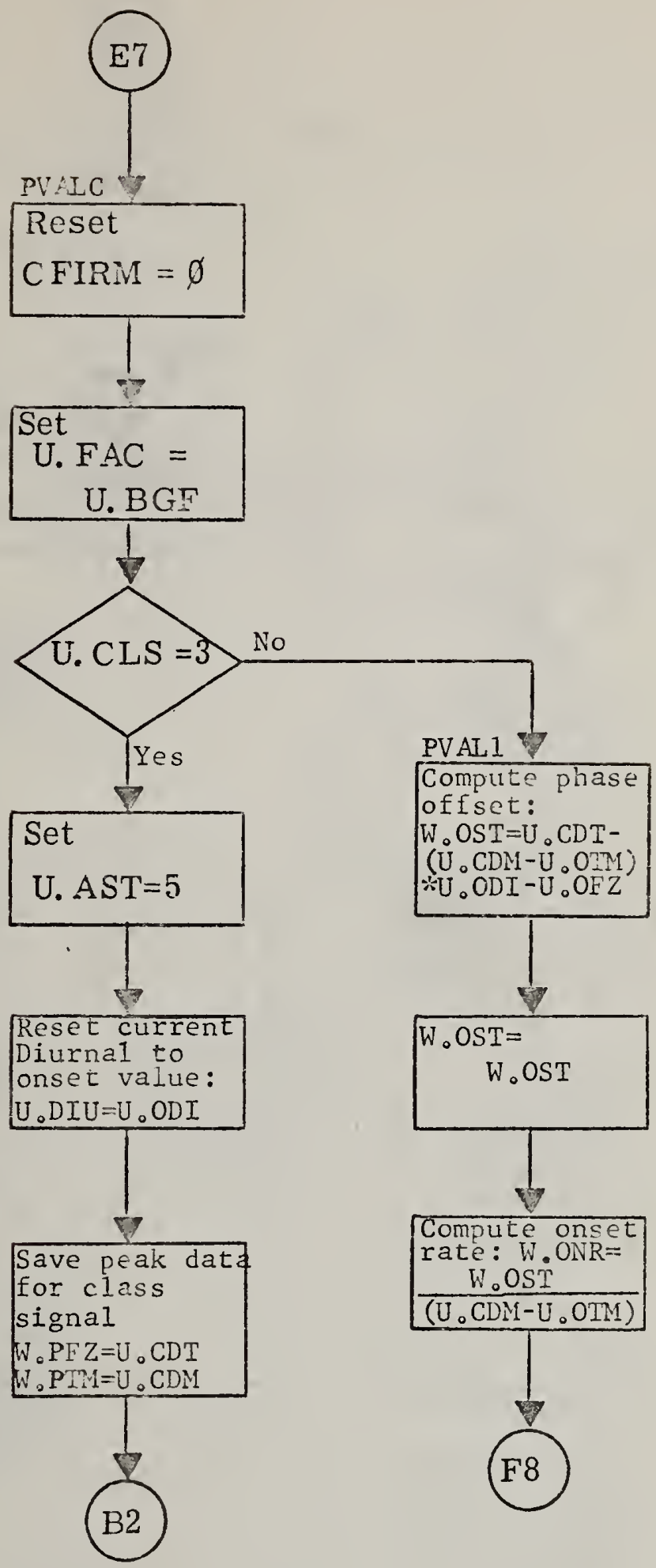




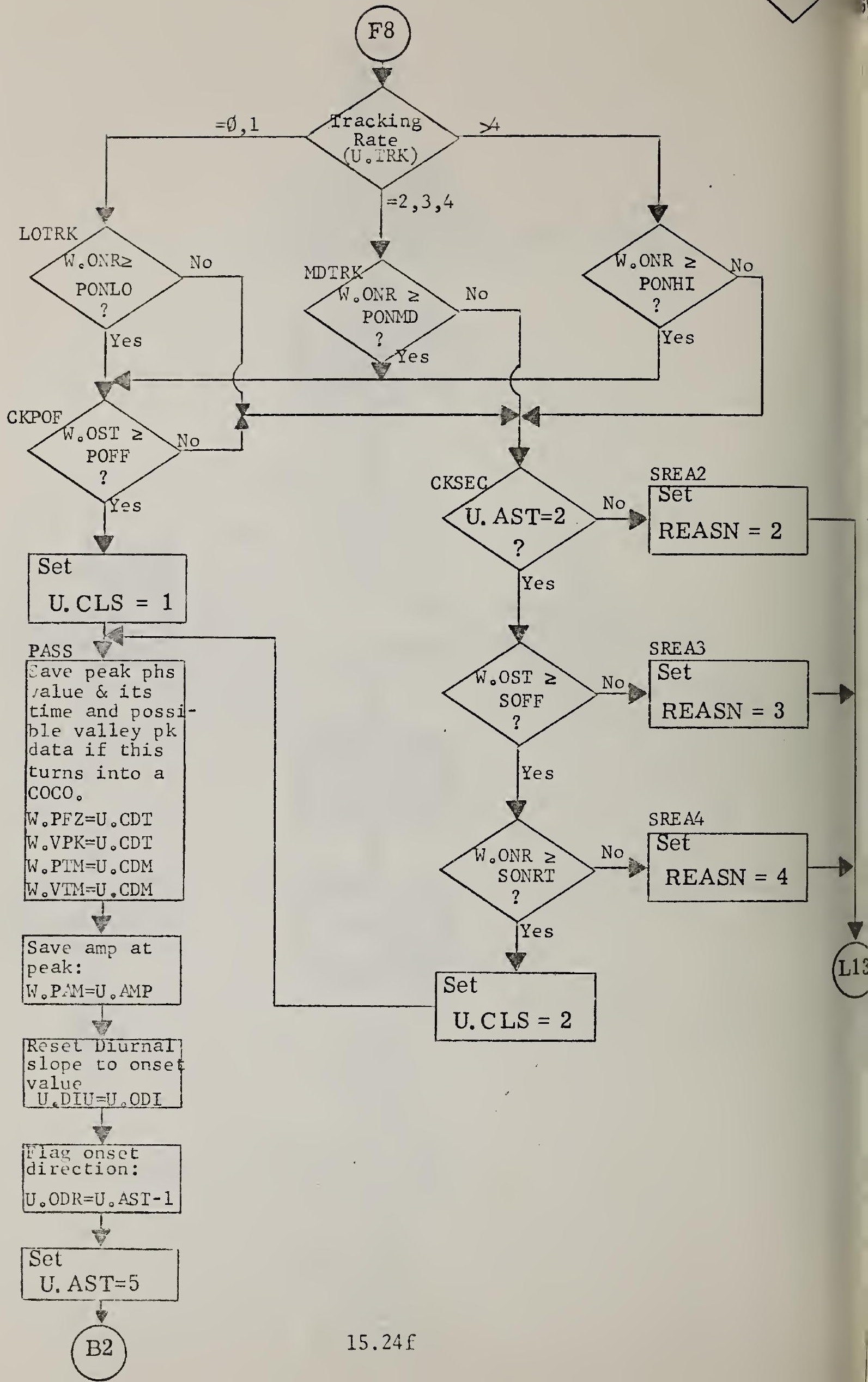




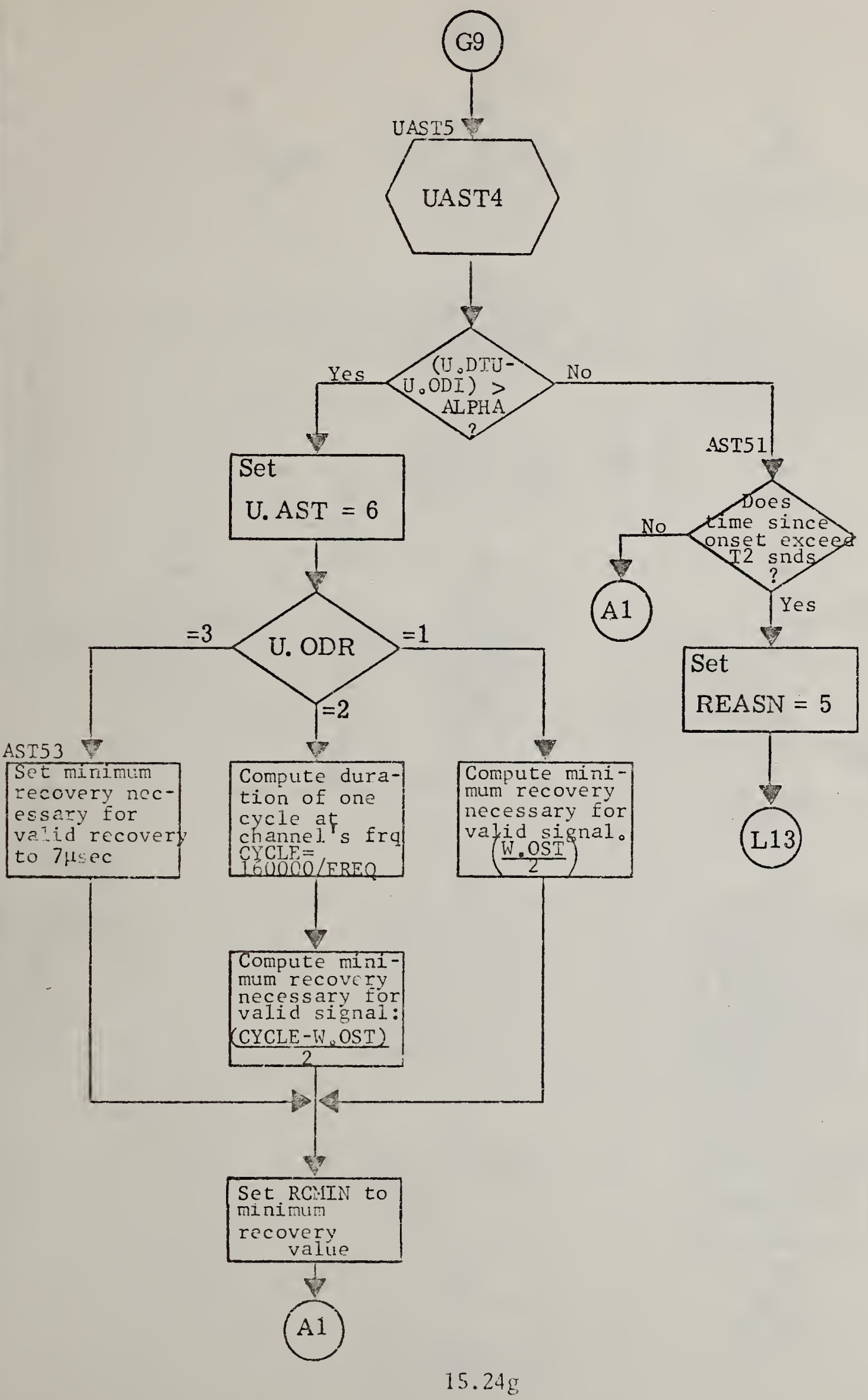




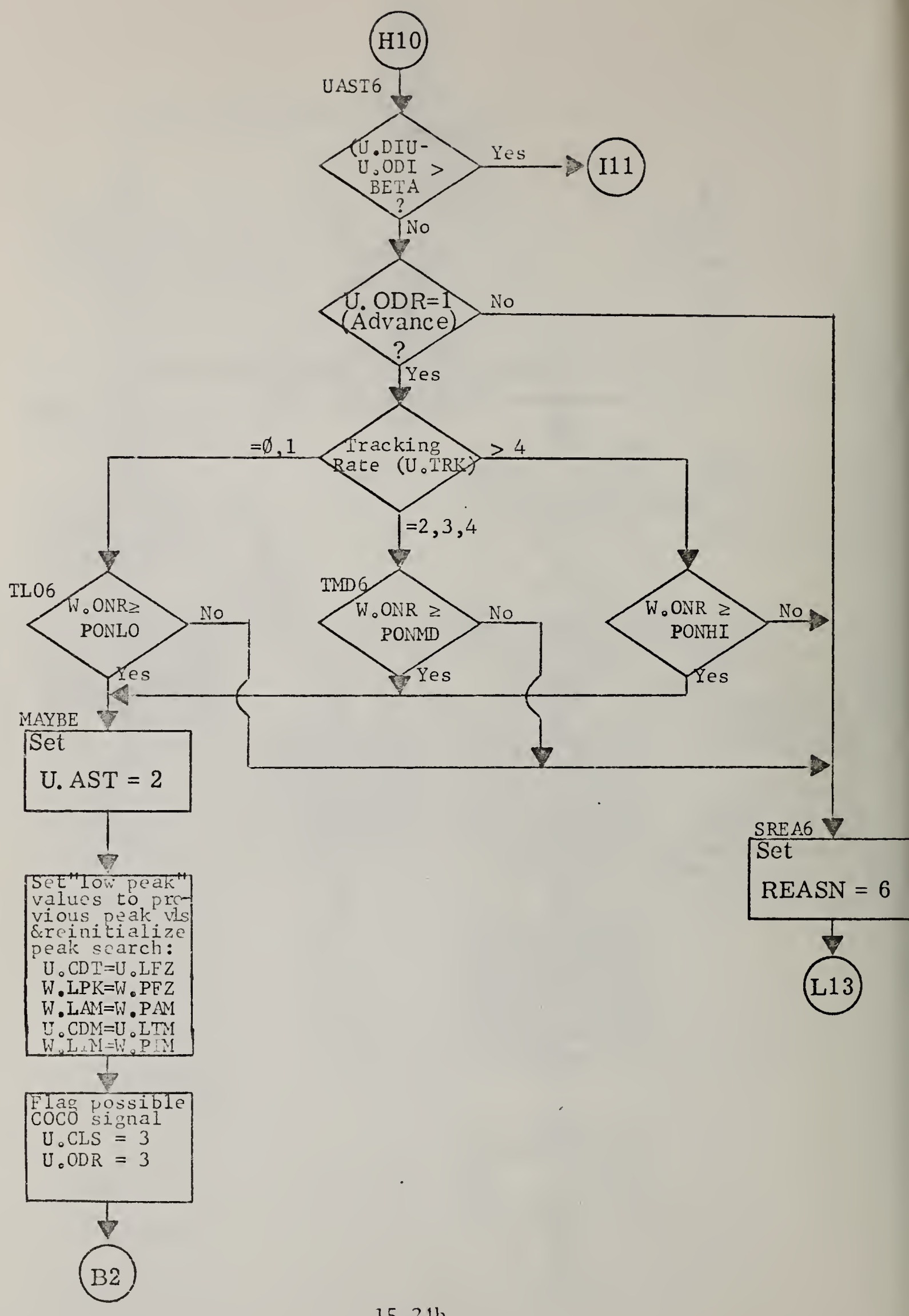


I11

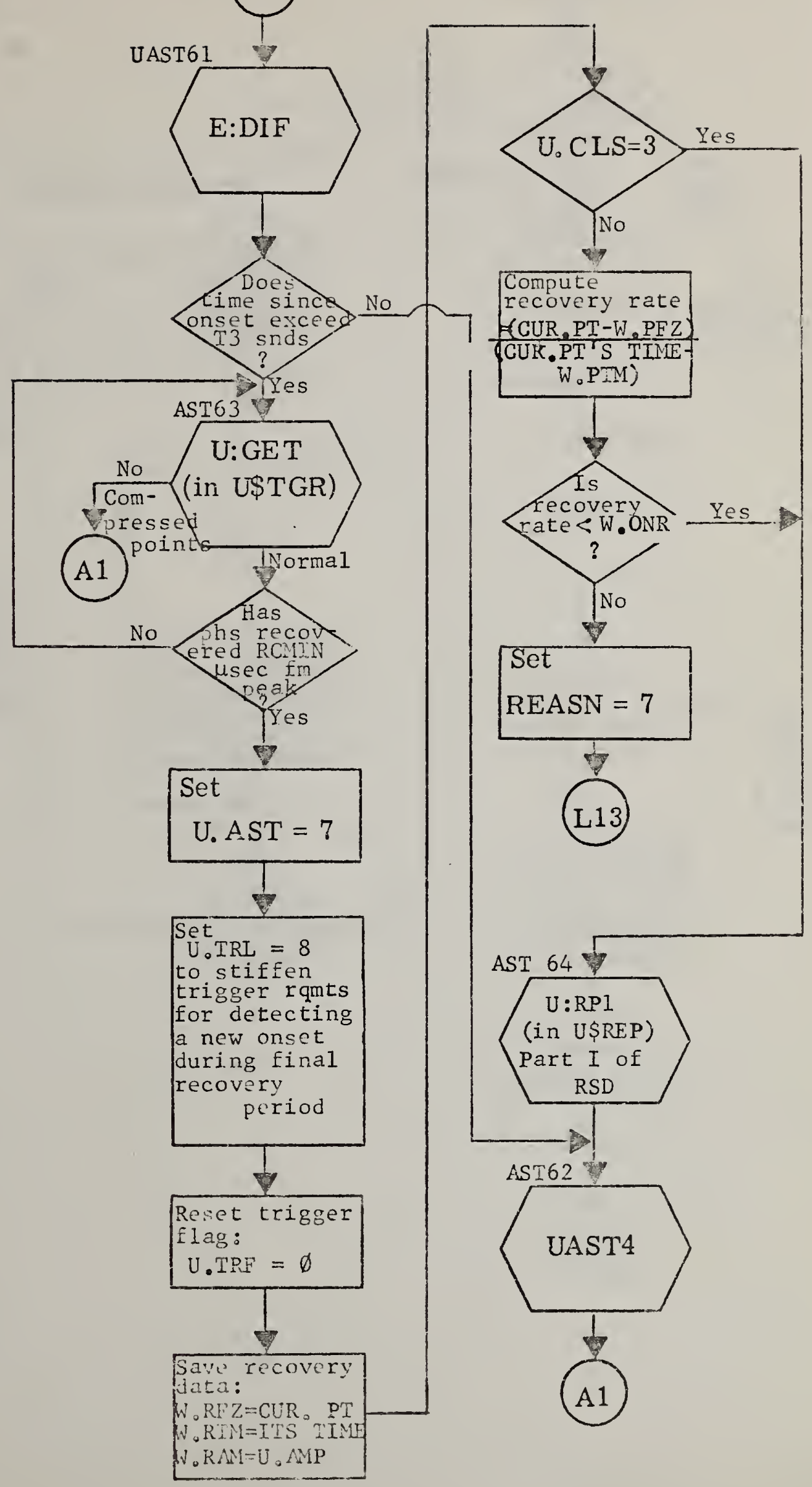




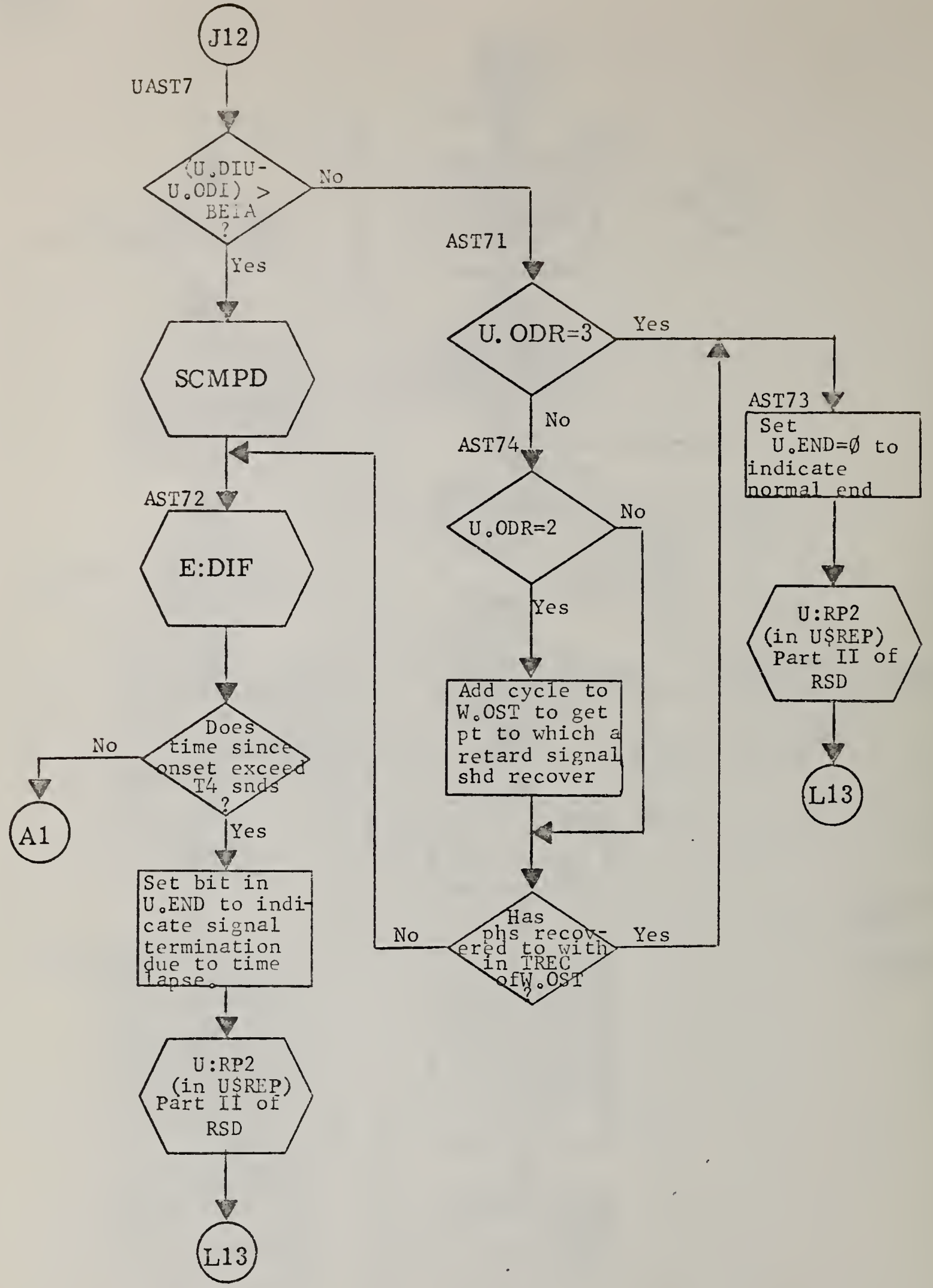



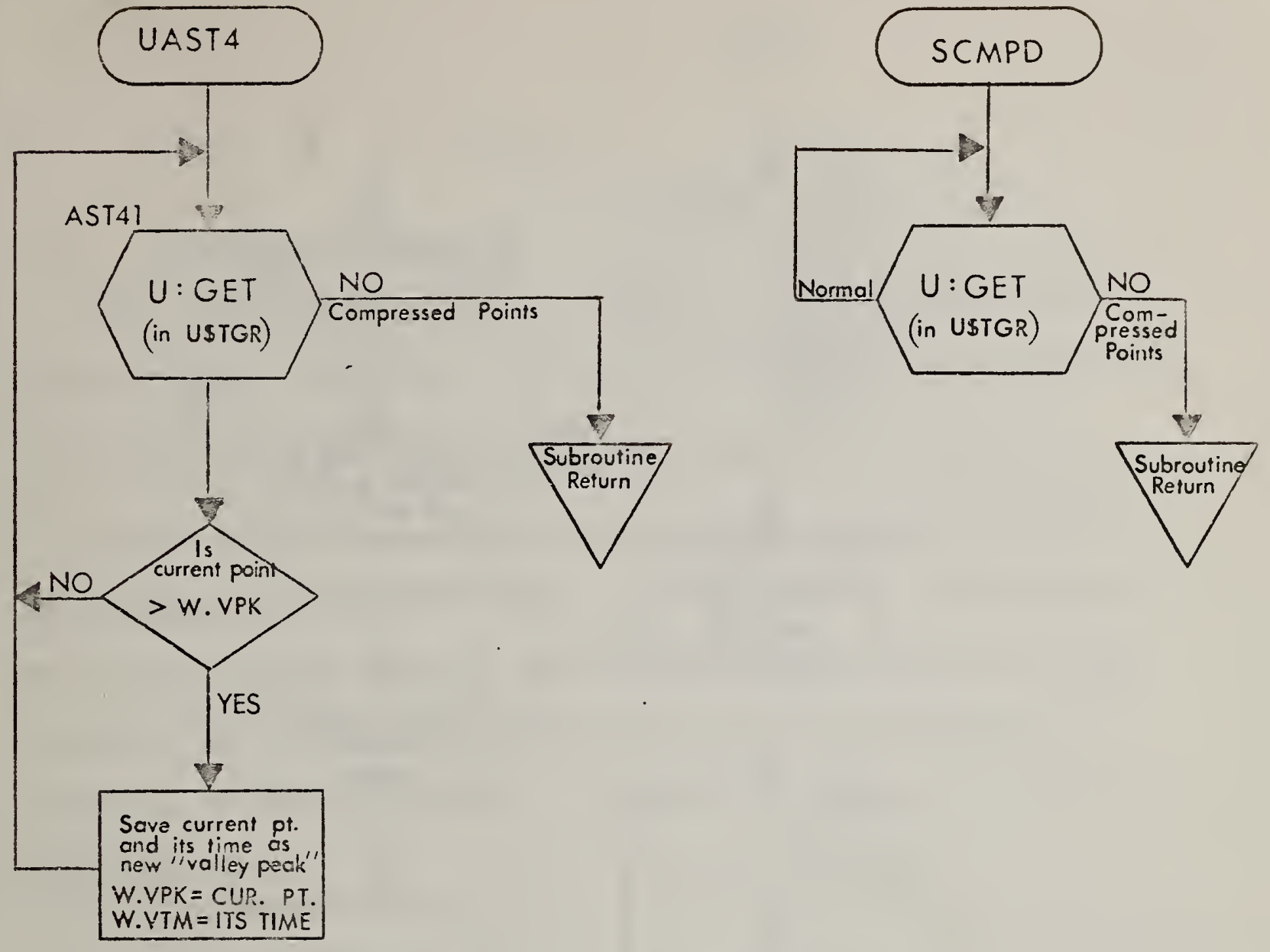


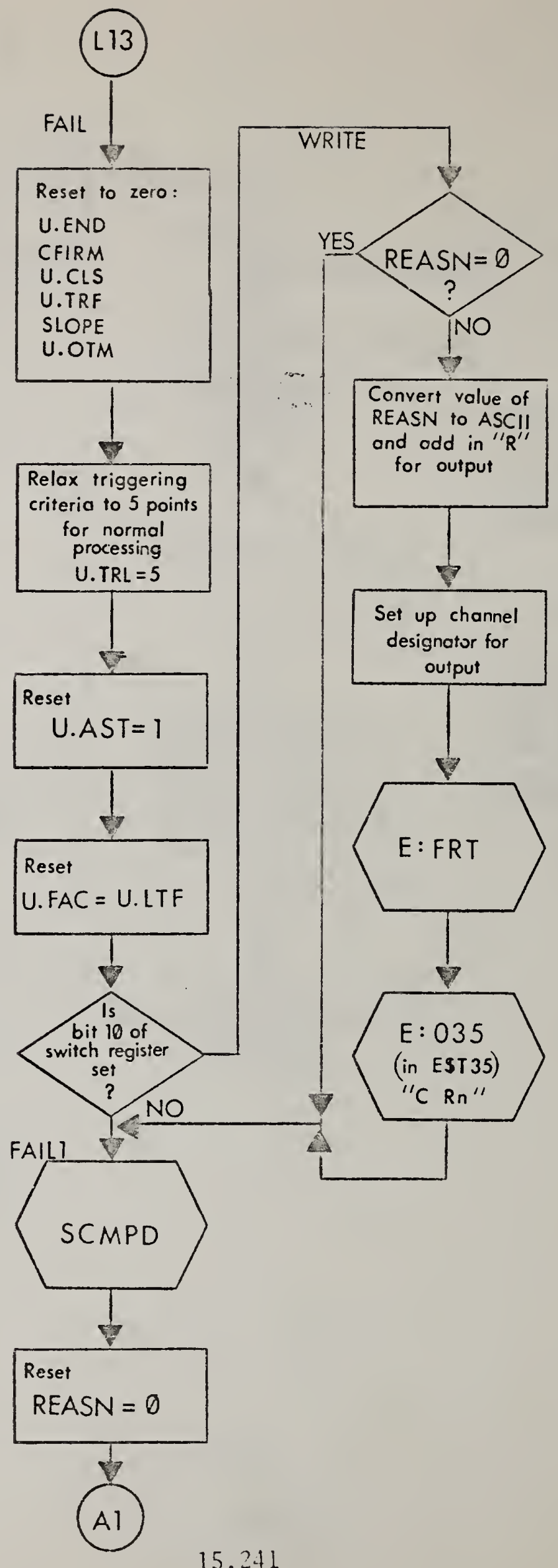


16. U\$DAT

16.1 Functional Description

U\$DAT contains no executable statements. It is a storage area where variables, flags, etc. are made available to the various applications programs in NUSTAD.

Because of the unique characteristics of this program in NUSTAD, the following write-up will differ slightly from those of the rest of the system. Section 16.2 will indicate the various formats which the parameters may assume. In Section 16.3, each of the parameters is described, and where applicable, a reference is made to its format as a subsection of 16.2 .

16.2 Data Formats

16.2.1 Long and Short Term Trend Estimates

The lease squares slope estimate to approximate the short term data trend and the long term estimate for the data trends use the following format:

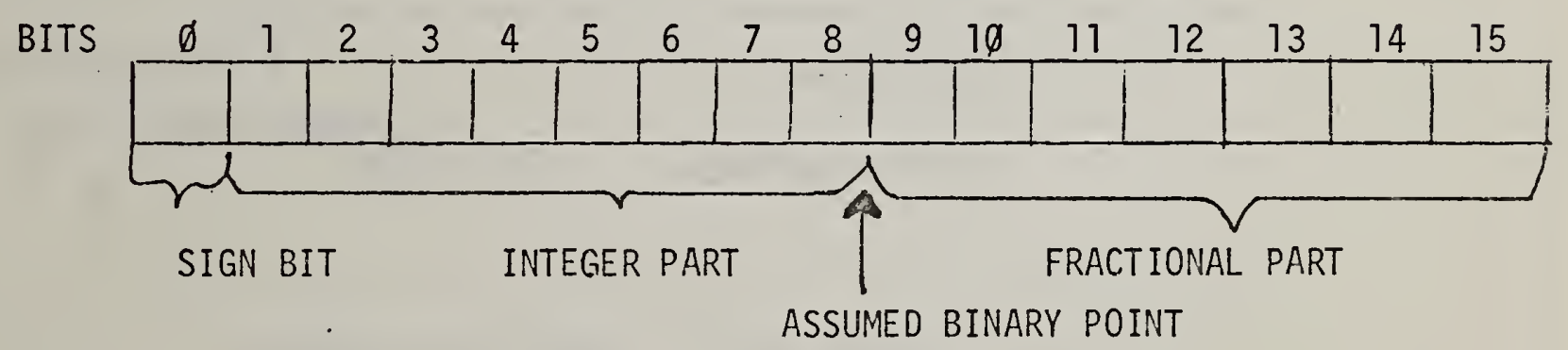

Figure 16.i Trend Estimates Format 16.1 


\subsubsection{Pure Fractional Values}

At several points in the programs, values which are purely fractional are required. These values use the following format:

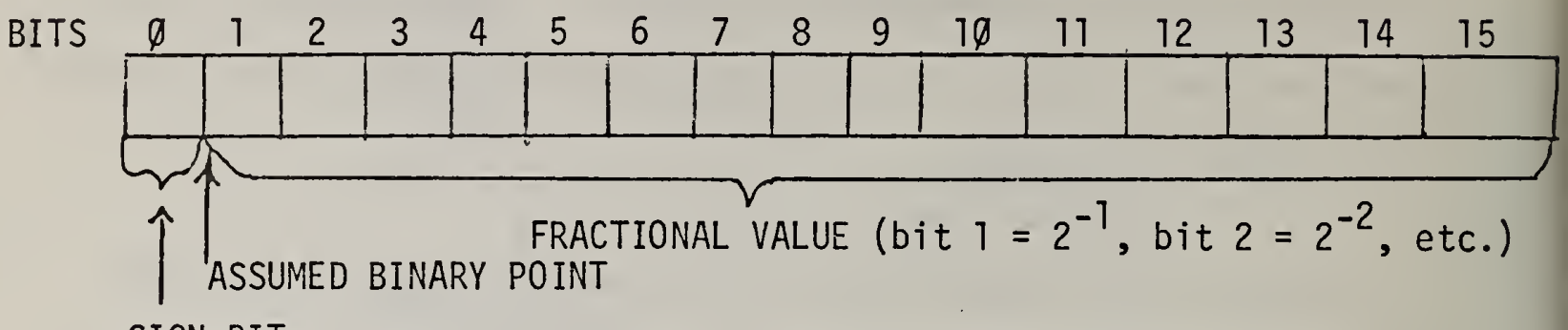

SIGN BIT

Figure 16.2 Fractional Value Format

\subsubsection{Phase Values}

The internal representation of the Phase values in the various programs, including such values as the phase offset between onset and peak of a waveform, and the standard deviation, is as follows:

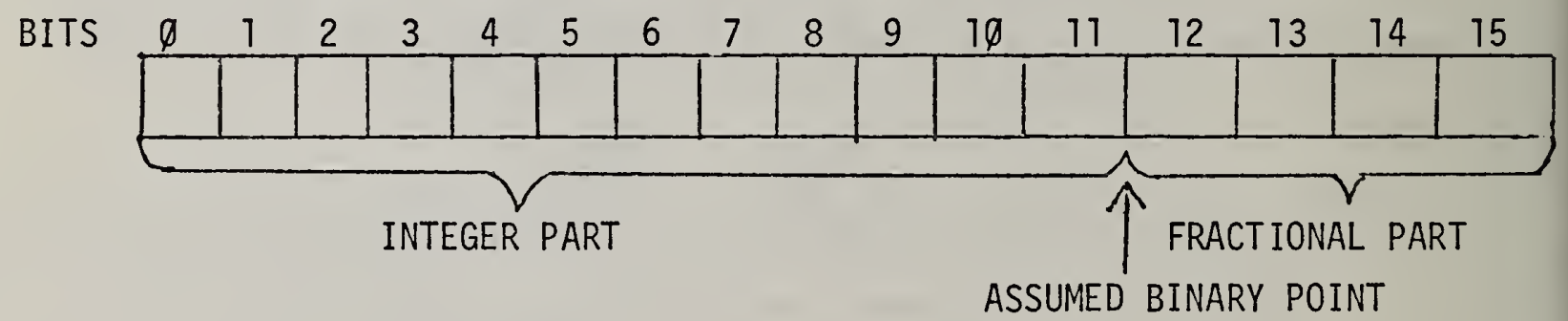

Figure 16.3 Phase Value Format 


\subsubsection{BCD Time}

The DOY/TOD group is input from the WTC via the MODCOMP III IOIS us ing the following format. Since the representation is compact, and the conversion from BCD to ASCII is straightforward, the internal representation of most time values is kept in this same format.

\section{FIRST WORD:}

BITS

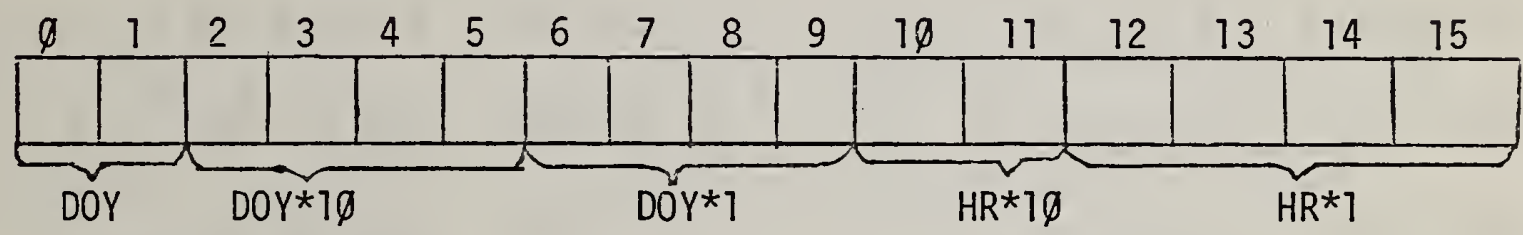

SECOND WORD:

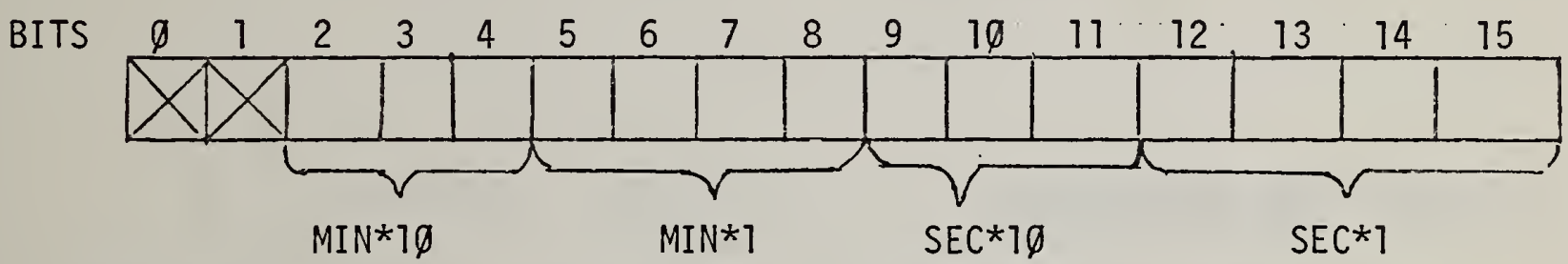

Figure 16.4 BCD Time Format 


\subsubsection{Binary Time}

For ease in computation, the $B C D$ time is occasionally converted to a form more compatible with binary arithmetic. This form consists of the equivalent of the TOD value represented as a binary count of seconds.

FIRST WORD:

$$
\text { SECOND WORD: }
$$

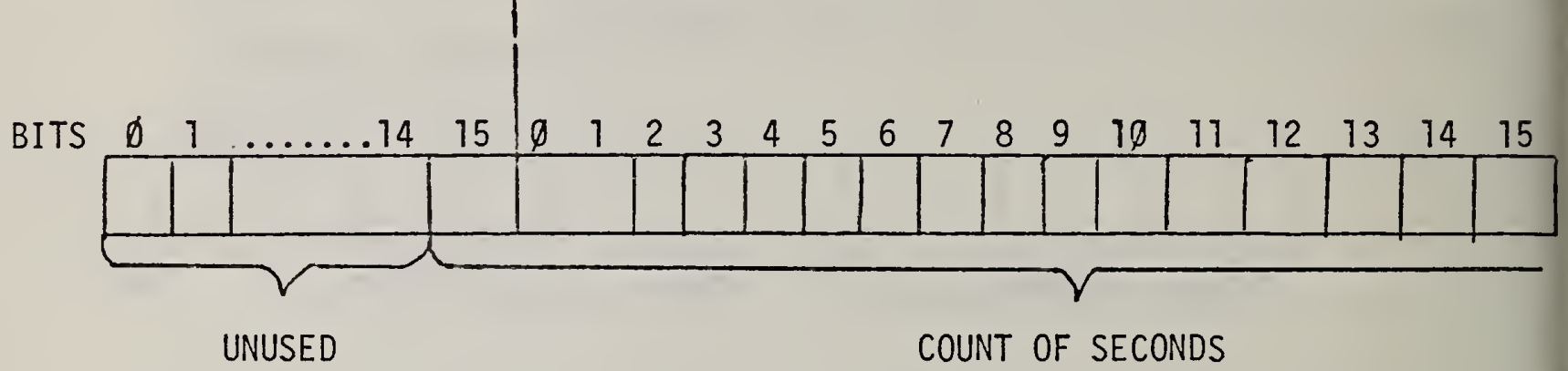

Figure 16.5

Binary Time Format

\subsubsection{Amplitude Values}

The internal representation of amplitude is an integer with a range from zero through 204710 corresponding to amplitude values of from $-2 \emptyset \mathrm{dB}$ to $+2 \varnothing \mathrm{dB}$. 


\subsubsection{U.!IRK Buffer Format}

After E.UIN collects ten seconds worth of data, it transfers that cata to the working buffer (U.WRK) and schedules the series of applications programs. The format of the data in U.WRK is as follows:

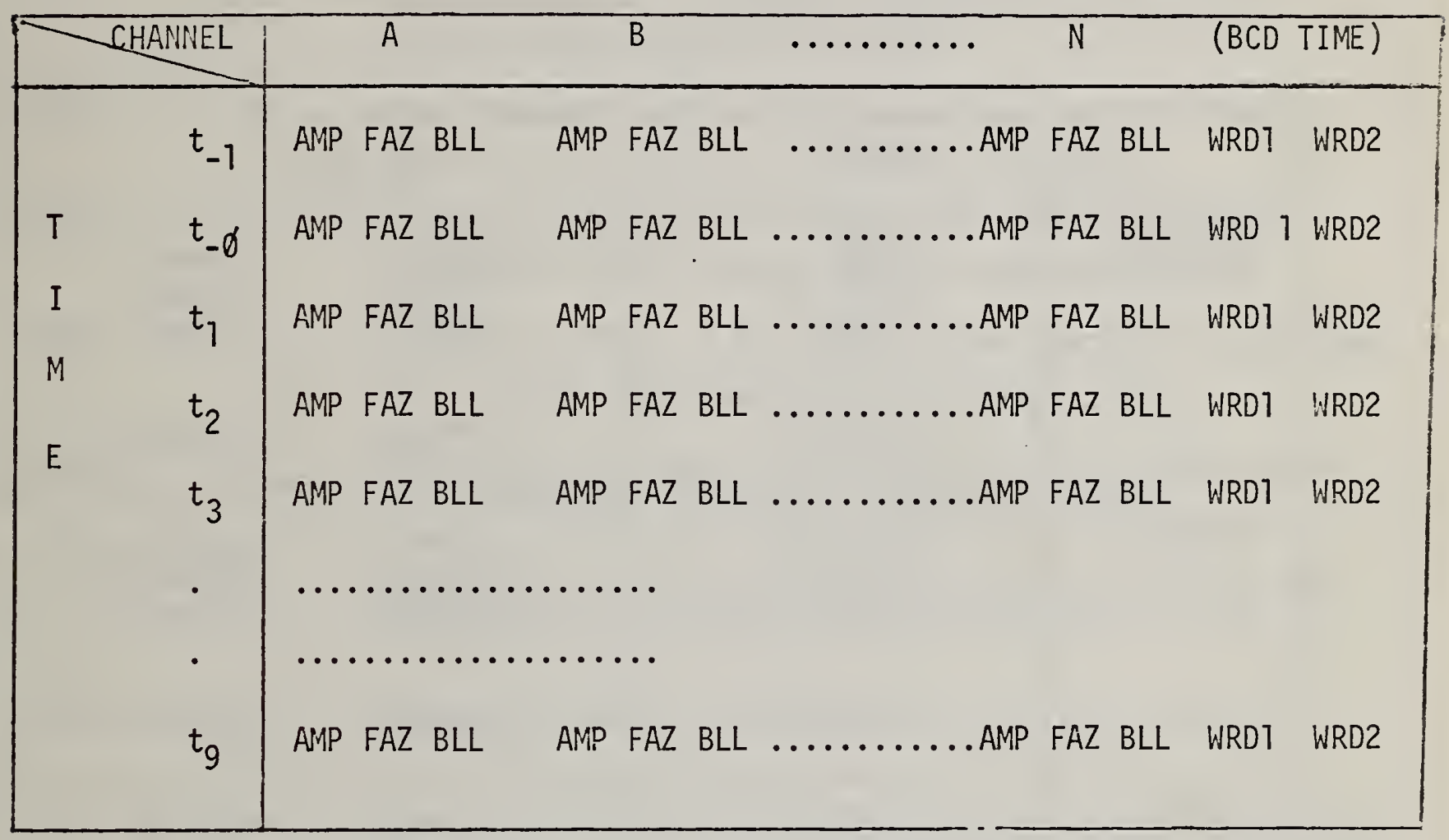

Chan.A AMP @ U.WRK; FAZ @ U.WRK+1; BLL @ U.WRK+2; etc.

Figure 16.6 Working Buffer (U.WRK) Format 


\subsubsection{U.CMP Buffer Format}

U.CMP is a buffer of compressed data points encountered during the current ten-second interval. Each entry requires three computer words; one for the phase value and two for the BCD time. Each of the fourteen channels has room for ten entries, resulting in a buffer $3 \times 14 \times 10$, or 420 words, arranged as follows:

\begin{tabular}{|c|c|c|c|}
\hline & PHASE & \multicolumn{2}{|c|}{ BCD TIME } \\
\hline CHANNEL A & 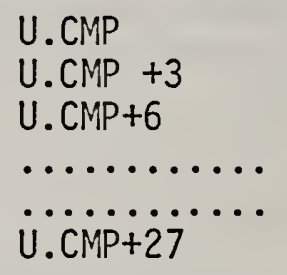 & $\begin{array}{l}\text { U.CMP+1 } \\
\text { U.CMP+4 } \\
\text { U.CMP+7 } \\
\text { U.CMP+28 }\end{array}$ & $\begin{array}{l}\text { U.CMP+2 } \\
\text { U.CMP+5 } \\
\text { U.CMP+8 } \\
\text { U.CMP+29 }\end{array}$ \\
\hline CHANNEL B & $\begin{array}{l}\text { U.CMP+3ð } \\
\text { U.CMP }+33 \\
\text { U.CMP+36 } \\
\cdots \cdots \cdots \cdots \\
\text { U.CMP+5 } . \cdots \cdots\end{array}$ & $\begin{array}{l}\text { U.CMP }+37 \\
\text { U.CMP }+34 \\
\text { U.CMP }+37 \\
\text { U.CMP }+58\end{array}$ & $\begin{array}{l}\text { U.CMP }+32 \\
\text { U.CMP }+35 \\
\text { U.CMP }+38 \\
\text { U.CMP }+59\end{array}$ \\
\hline CHANNEL C & 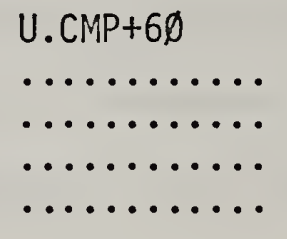 & U.CMP+61 & U.CMP+62 \\
\hline
\end{tabular}

Figure 16.7 Compressed Point Buffer (U.CMP) Format 
16.3 Output Parameter Descriptions

PARAMETER SIZE DESCRIPTION

U.TRK

14 Current setting for sensor channel tracking rate. value between $\emptyset$ and 9 inclusive.

U.GAN $14 \quad$ Current setting for sensor channel RF gain. Vālue between $\emptyset$ and 24 inclusive.

U.BLL $14 \quad$ Current setting for sensor channel blanking level. Value between $\emptyset$ and 63 inclusive.

U.DIU 14 The diurnal slope, or long term data trend, estimate. Format as in 16.2.1 as microseconds/minute.

U.LTF $\quad 1 \quad=\varnothing 666_{16} ;(.951 \emptyset)$. The longer time constant factor from the digital filter used in determining U.DIU.

U.BGF $\quad 1=\varnothing \operatorname{CCCC}_{16} ;\left(.1 \varphi_{1 \emptyset}\right)$. The shorter time constant factor from the digital filter used in determining U.DIU.

U.FAC $\quad 14$

The current factor, set to either U.LTF or U.BGF, used in the digital filter for determining U.DIU.

U.LST $\quad 140$

A wrap-around buffer of ten-second phase averages used in computing the least squares slope estimate (see Section 13.4.1).

U.LSP

1

A pointer to the current row in U.LST (see Section 13.4.1).

U.SLP $\quad 14$

The current short term phase trend estimate determined by the least squares slope computation described in Section 13.3 .

U.WRK

484

The working buffer into which the data from the AIS and the DOY/TOD group from the IOIS are transferred for use by the applications programs (see Section 16.2.7).

U.CTM

The $B C D$ time value read into the last two words of U.WRK; hence, the current time of the current ten-second interval. 


\subsection{Output Parameter (continued)}

\section{PARAMETER SIZE DESCRIPTION}

U.CMP

$42 \emptyset$

U.CPP

U.CPC

U.CYC

14

U.CCN

U.IHB

U.RTY

U.CST

U.AST
The buffer of compressed data points (phase and BCD time) for each channel encountered during the current ten-second interval (see Section 16.2.8).

14 A table of pointers to the first word of each sensor channel's partition of U.CMP.

A counter for the number of compressed data points encountered on each sensor channel during the current ten-second interval.

14 A flag, set to $+5 \emptyset$ or $-5 \emptyset$ microseconds (see Section 16.2.3) when a phase shift is induced by NUSTAD to indicate the direction. of the shift. See E:UIN for complete details.

14 Accumulated sum of U.CYC to indicate the total effect of cycle shifts induced by NUSTAD.

Flag bit (BIT $\emptyset$ ) is set to inhibit processing on a sensor channel when a maintenance of INE condition exists on that channel.

14 Counter for consecutive ten-second intervals of "good" data required to bring a channel to the UP state.

The channel's processing status, based on the results of the amplitude monitoring section of U:RTA;

$=1$ for channel UP

$=2$ for channel COASTING, with NO REPORT

$=3$ for channel COASTING, REPORTED on logging teleprinter

$=4$ for channel DOWN

The channel's waveform analysis status, determined by the point in the signal to which processing has progressed;

$=1$ while looking for onset

$=2$ while looking for peak in the advance direction

$=3$ while looking for peak in the retard direction

$=5$ while awaiting recovery to begin

$=6$ while looking for valid recovery point

$=7$ while looking for end of signal 
16.3 Output Parameter Descriptions (continued)

\begin{tabular}{lll} 
PARAMETER & SIZE & DESCRIPTION \\
\hline U.CLS & $\begin{array}{l}\text { The class of the waveform being processed; } \\
=1 \text { for those meeting primiary criteria } \\
=2 \text { for those meeting secondary criteria } \\
=3 \text { for multiple peak signals }\end{array}$
\end{tabular}

U.END

14 Flag to indicate how processing terminated on waveform just completed;

Bit $\emptyset=\emptyset$ Normally

$=1$ Signal terminated due to channel going down

Bit $1=\emptyset$ Normally

$=1$ Time Timit T4 exceeded, signal terminated

Bit $2=\emptyset$ Normal1y

$=1$ New onset terminates former signal

U.OTM

28

Signal onset time, binary (Section 16.2.5)

U.DTM

2

Dummy binary time used by E\$DIF (Section 16.2.5)

U.OTB

28

Signal onset time, $B C D$ (Section 16.2.4)

U.OAM

14

Signal amplitude at onset (Section 16.2.6)

U.OFZ

14

Phase value at onset (Section 16.2.3)

U.ODI

14

Long term phase trend estimate at onset (Section 16.2.1)

U.ODR

14

Direction of onset;

$=1$ for advance

$=2$ for retard.

$=3$ for advance, multiple peak

U.OSG

14

Standard deviation (U.SIG) at onset (Section 16.2.3)

W.PFZ

14

Phase value at peak (Section 16.2.3)

W.PTM

$B C D$ tiline of peak (Section 16.2.4)

W.PAM

Signal amplitude at peak (Section 16.2.6)

W.OST

14

Phase offset, onset to peak (Section 16.2.3)

W.ONR

14

Average rate of onset, onset to peak (Section 16.2.3) 


\subsection{Output Parameter Descriptions (continued)}

$\begin{array}{lll}\text { PARAMETER } & \text { SIZE } & \text { DESCRIPTION } \\ \text { W.LPK } & 14 & \begin{array}{l}\text { Phase value at first peak of multiple peak signal } \\ \text { (Section 16.2.3). }\end{array} \\ \text { W.LTM } & 28 & \begin{array}{l}\text { BCD time at first peak of multiple peak signal } \\ \text { (Section 16.2.4). }\end{array} \\ \text { W.LAM } & 14 & \begin{array}{l}\text { Signal amplitude at first peak of multiple peak signal } \\ \text { (Section 16.2.6). }\end{array} \\ \text { W.VPK } & 14 & \begin{array}{l}\text { Phase value at valley peak of multiple peak signal } \\ \text { (Section 16.2.3). }\end{array} \\ \text { W.VAM } & 14 & \begin{array}{l}\text { Signal amplitude at valley peak of multiple peak signal } \\ \text { (Section 16.2.6). }\end{array} \\ \text { W.VTM } & 28 & \begin{array}{l}\text { BCD time at valley peak of multiple peak signal } \\ \text { (Section 16.2.4). }\end{array} \\ \text { W.RFZ } & 14 & \begin{array}{l}\text { Phase value at valid recovery (Section 16.2.3). } \\ \text { BCD time of valid recovery (Section 16.2.4). }\end{array} \\ \text { W.RTM } & 28 & \begin{array}{l}\text { Signal amplitude at valid recovery (Section 16.2.6). } \\ \text { W.RAM }\end{array} \\ \text { U.AMP } & 14 & \begin{array}{l}\text { Estimate of current signal amplitude based on the } \\ \text { value from the last input of each ten second interval, } \\ \text { (Section 16.2.6). }\end{array}\end{array}$


17. U\$REP

\subsection{Functional Description}

When a signal meeting reporting criteria is encountered by U:WFA, a message called the REPORTABLE SIGNAL DESCRIPTION is generated in three parts for the station log. Each part is called in sequence as conditions dictate.

\subsubsection{U:RPI (Generator for Part I of RSD)}

When U:WFA sets U.AST to seven, it considers the signal reportable and calls U:RP1 to generate the first part of the RSD and start it on its way to the ASR 28, the logging teleprinter. The first part of the RSD supplies the operator with a relatively detailed description of the waveform to the point where the recovery is considered valid. This information is intended for the operator's use only. After the message is generated and transferred to the ASR 28 output buffer via calls to $E: 028$, control is returned to the calling program.

\subsubsection{U:RP2 (Generator for Part II of RSD)}

When signal processing terminates for a signal in progress, U:WFA wi11 ca11 U:RP2 to generate a followup to the message generated by U:RP1. This followup is the second part of the RSD and supplies the operator with the same type of information formerly output, but concerning the signal termination. When this part of the RSD is transferred to the output buffer, a flag bit is set in E.28F to signify that U:RP3 is to be scheduled 
the next time the output buffer in E\$T28 is empty. This procedure is a precaution against the lengthy $U: R P 3$ message becoming disturbed in the wrap-around designed output buffer.

After the call to E:028 during which R:RP 3 is effectively scheduled, control is returned to the calling program.

\subsubsection{U:RP3 (Generator for Part III of RSD)}

Each time the output buffer in E\$T28 becomes empty, a flag bit is checked to determine whether there is a need to schedule U:RP3 (see Section 9.1.2). If the bit is set, due to a previous call to E:028 by either U:RP2 or U:RP3, U:RP3 is scheduled in the system scheduler. Control is thereafter made to $U: R P 3$ from $E: S K D$.

Whereas Parts I and II of the RSD were designated for operator information, Part III is formatted for transmission over standard communications lines. Therefore, a series of null characters is generated before and after Part III of the RSD to create a leader on the data tape, and numeric characters are spelled out ("ONE" for 1, "TWO" for 2, etc.). When U:RP3 transfers its message to the ASR 28 output buffer in E\$T28, it sets the flag bits in E.28F to cause U:RP 3 to be rescheduled after the output buffer again becomes empty. In this way, only one Part III RSD message may be generated at a time.

When $U: R P 3$ is finally entered with no further message to generate, a final call to E:028 is made to transmit two "LTR" characters without the flag bit set in E.28F. 
U:RP3 returns control to the scheduler each time it completes execution.

\subsection{Called Subroutines}

SUBROUTINE PROGRAM FUNCTION

\section{E:FRT E\$FRT Reformats BCD time from the WTC to ASCII for output.}

E:BAD E $\quad$ Converts binary value to ASCII decimal characters.

E:FMF $\quad$ E\$FMF Reformats phase values from internal binary representation to ASCII decimal for output.

E:FAM MSSTD Reformats amplitude values from internal binary representation to ASCII decimal $\mathrm{dB}$ for output.

E:FCT E\$FRT Reformats BCD time from memory to ASCII for output.

E:028 E\$T28 Transiates output message to BAUDOT code and stores in buffer before transmission to the ASR 28.

E:COT E\$DIF Converts the BCD time characters to the binary count of seconds.

E:SKD E\$SKD Operating system scheduler entry point.

$E: B D C \quad E \$ B D C \quad B$ Binary to $B C D$ (decimal) conversion routine.

E:DIF E\$DIF Computes the time difference between onset and later point in time.

\subsection{Data Formats}

\subsubsection{U:RP1 Message Format}

Part I of the RSD is generated by U:RP1 at the time when a reportable waveform is considered valid. The data applicable to the key points of the 
waveform up to that point are output in tabular form as shown in Figure 17.1. The following describes the format of the message:

LINE 1:

DDD TITTTT REPORTABLE SIGNAL DESCRIPTION, DET NNN

where: $\underline{D}$ indicates a digit of $\mathrm{DOY}$

I indicates a digit of TOD

$\underline{N}$ indicates a digit of detachment number 


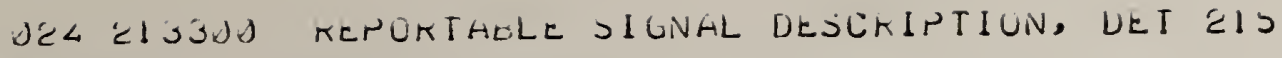
bic

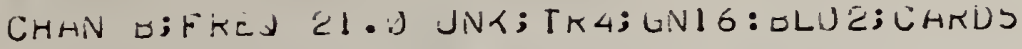

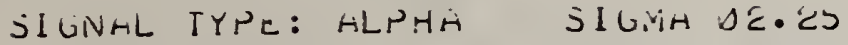

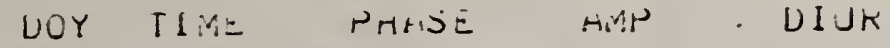

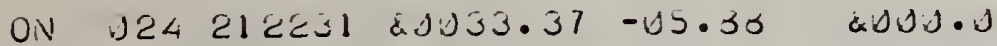

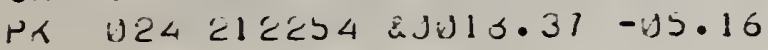

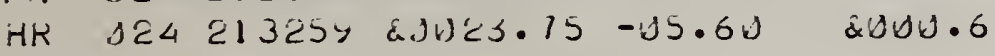

OFFSET: MSIS.DU

ONSET KHTE: WJY.1

U25 1041 DE REPCRTAELE SIGNAL DESCRIPTION, DET 215 013

CHAIN C;FREO 21. UNR;TKL;GNI 5: ULD4;CARDI

SIGNAL TYPE: BRAVO SIGMA DU. 56

$$
\text { DOY TIME PHASE HIP DIUR }
$$

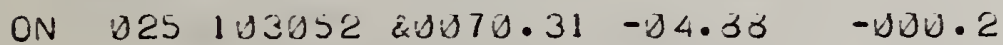

PK D2S 103120 \&JणY1.43-04.6I

HK DCS 104055 \&U105.93 -04.3ర \&UDU.6

OFFSET: JU21.13

ONSET RATE: O 45.4

024223921 REPORTAGLE SIGNAL DESLRIPTION, DET ZIS (j) 55

CHAN G;FREJ 21.U UNK; TRS;GN16:DLU2:CARDS

SIGNAL TYPE: DELTA SIGIA JU.SU

$$
\text { DOY TIVIE PHASE AIVP DIUR }
$$

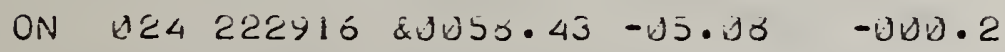

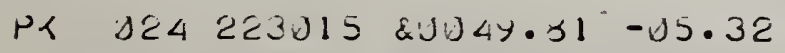

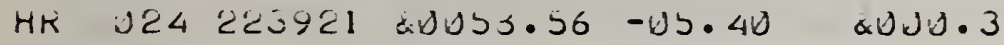

OFFSET: IU⿴囗⿰丿㇄丁

ONSET KATE: UUठ.6

Figure 17.1 Three Examples of Part I of the RSD Message 


\section{LINE 2:}

NNN

where: $\underline{N}$ indicates a digit of a three digit serial identification number, used to associate the three parts of the RSD with the signal.

\section{LINE 3:}

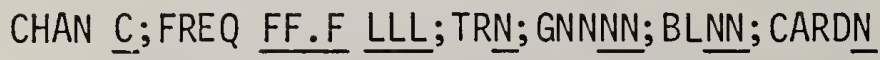

where: $\underline{C}$ indicates the sensor channel reporting

F indicates a digit of the sensor frequency

$\underline{L}$ indicates a letter of the call letters for that frequency, UNK used when no known call letters correspond to that frequency.

N indicates digits used to indicate the current values associated with the sensor's TRACKING RATE, RF GAIN, BLANKING LEVEL, and CARDIOID CONFIGURATION.

\section{LINE 4:}

SIGNAL TYPE: YYYYYY

SIGMA SS.SS

where: $\underline{Y}$ indicates a letter in the signal type designator: (ALPHA, BRAVO, COCO, or DELTA)

$\underline{S}$ indicates a digit of the standard deviation of the input signal at onset.

LINE 5:

DOY TIME PHASE AMP DIUR

where: DOY is the column heading for the Day of Year data TIME is the column heading for the Time of Day data PHASE is the column heading for the Phase value data AMP is the column heading for the signal amplitude data DIUR is the column heading for the diurnal slope estimate data. 
LINE 6: (data from onset)

ON DDD TTTTTT PPPPP.PP AAA.AA RRRR.R

where: ON indicates the line covers onset

$\underline{D}$ indicates a digit of DOY

I indicates a digit of TOD

$\underline{P}$ indicates a digit of phase value (microseconds)

A indicates a digit of amplitude value $(d B)$

$\underline{R}$ indicates a digit of diurnal slope estimate (mircoseconds/minute)

LINE 6a: (data for the first peak of COCO signal, not used with other tyeps)

IP DDD TTTTTT PPPPP.PP AAA.AA

where: IP indicates the line covers the first peak

$\underline{D}$ indicates a digit of DOY

I indicates a digit of TOD

$\underline{P}$ indicates a digit of phase value (microseconds)

A indicates a digit of amplitude value $(d B)$

LINE 6b: (data for the valley peak of COCO signal, not used with others)

2P DDD TTTTTT PPPPP.PP AAA.AA

where: $2 P$ indicates the line covers the valley peak

D indicates a digit of DOY

I indicates a digit of TOD

$\underline{P}$ indicates a digit of phase value (microseconds)

A indicates a digit of amplitude value (dB) 
LINE 7: (data from signal peak)

PK DDD TTTTTT PPPPP.PP $\underline{\text { AAA.AA }}$

where: PK indicates the line covers the signal peak

$\underline{D}$ indicates a digit of DOY

I indicates a digit of TOD

$\underline{P}$ indicates a digit of phase value (microseconds)

A indicates a digit of amplitude value $(\mathrm{dB})$

$\underline{R}$ indicates a digit of diurnal slope estimate (microseconds/minute)

LINE 9:

OFFSET: NNNN.NN

where: $\underline{N}$ indicates a digit of the computed phase offset, including the compensation for the diurnal slope estimate at onset, (microseconds)

LINE 19:

ONSET RATE: NNN.N

where: $\underline{N}$ indicates a digit of the computed average rate of onset between onset and peak, (microseconds/minute) 


\subsubsection{U:RP2 Message Format}

Part II of the RSD is generated by U:RP2 when processing is terminated on a current signal. Data concerning the reason for termination and applicable to the time of termination are output in the same basic format as Part I of the RSD. Figure 17.2 displays three examples of Part II of the RSD, and the following explains the format:

LINES 1 through 5 are the same as Part I.

LINE 6: (data from the time of signal termination)

FR DDD TTTTTT PPPPP.PP AAA.AA RRRR.R

where: FR indicates the line covers the time of final recovery

D indicates a digit of DOY

I indicates a digit of TOD

$\underline{p}$ indicates a digit of phase value (microseconds)

A indicates a digit of amplitude value $(\mathrm{dB})$

$\underline{R}$ indicates a digit of diurnal slope estimate (microseconds/minute)

\section{LINE 7:}

\section{SIGNAL TERMINATED:}

where: $M$ is a letter from one of the following: NORMALLY for normal signal termination TIME LAPSE for signals that exceed one hour SIGNAL LOST for signals when amplitude drops out NEW ONSET for signals which lead into a new signal 
JE4 - ILLEI hEPURTMELE JIGNAL UESUKIPTIUN, DET ¿IJ

ง. 32

CHMN B;FKEJ 21.J JNK; TK4; LNI6: ELU2;LARDS

SIGNHL TYPE: HLHHH SIU.YH JE.

DOY TIME PHASE HIP DIJK

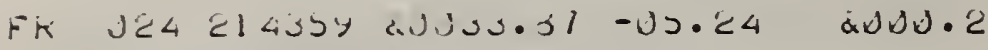

SIGNAL TERMIINATED: NURMALLY

D2S 11 \$343 KEPORTAULE SIGNAL UESCKIPTION, DET 215 1) 3

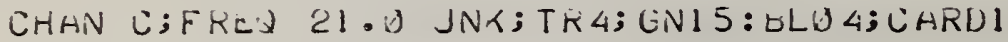

SIGNHL TYPE: UKAVO SIGVIA DU. 56

DOY TIME PHASE AMP DIUK

$F F$ J2S $11 \cup 339$ \&J116.UJ - 4.36 \&UDU.U

SIGNAL TEKMINATED: NORMALLY

O24 ¿24531 KEPORTABLE SIGNAL DESCRIPTION, DET 215 005

CHAN E;FREJ 21.U JNK; TR5;GN16: ULU2;CAKUS

SIGNHL TYPE: DELTH SIGMA UD.SU

DOY TIME PHASE AMP DIUK

FR 024224513 \&UUSउ.12 - U4.43 \&300.U

SIGNAL TERMINATED: NORMALLY

Figure 17.2 Three Examples of Part II of the RSD Message 


\subsubsection{U.RP3 Message Format}

Whenever U:RP2 or U:RP3 transmits part of an RSD message to E:028 for output to the ASR 28 teleprinter, they set a flag bit in E.28F to cause E:T28 to reschedule U:RP3 when the output buffer next becomes empty. Part III of the RSD message is then generated using the following format, as illustrated in Figure 17.3.

\section{LINE 1:}

RSD (serial number)

where: The serial number is a three digit value used to identify the signal on this sensor chanriel. It is initialized each time NUSTAD is reloaded and incremented for each new RSD message generated. It allows the operator to associate the three parts of the RSD with the same signal.

(Class), (Type), (Frequency \& Cal1 Letters), (Path),DOY)

where: Class is either A or B corresponding to whether Class A or Class B criteria were met.

Type is one of the following: ALPHA, BRAVO, COCO, or DELTA

Frequency is the sensor frequency setting, given digitally, and the call letters are those associated with that frequency.

Path is either FRONT or BACK, based on the cardioid pattern. DOY is the three digit DOY.

\section{LINES 3 \& 4: (Paragraph A)}

A. (Onset time), (Offset), (Direction)

(Onset Rate), (Onset Amp Titude) 


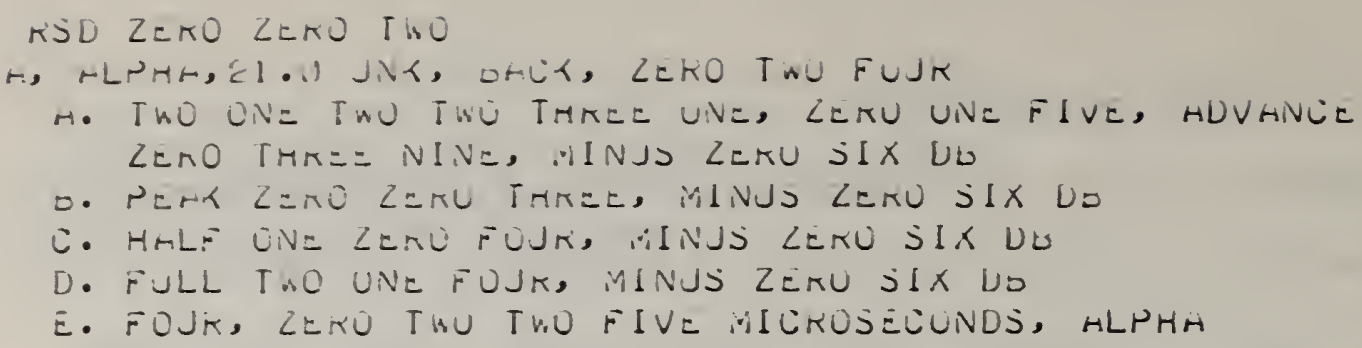

Figure 17.3 Three Examples of Part III of the RSD Message 
where: Onset time is the TOD at onset.

Offset is the phase offset computed between orset and peak, given in three digits to the nearest microsecond.

Direction is either ADVANCE or RETARD, the direction of onset.

Onset Rate is the average rate of onset to peak, given in three digits to the nearest microsecond per minute.

Onset Amplitude is the signal amplitude at onset to the nearest $\mathrm{dB}$.

LINE 5: (Paragraph B)

B. (Time from onset to peak), (Peak Amplitude)

where: Time from onset to peak is given as three digits representing tenths of minutes.

Peak Amplitude is given to the nearest $\mathrm{dB}$.

LINE 6: (Paragraph C)

C. HALF (Time fron onset to valid recovery), (Half recovery Amplitude)

where: Time is in three digits of tenths of minutes.

Amplitude is given in $\mathrm{dB}$.

LINE 7: (Paragraph D)

D. FULL (Time from onset), (Amplitude at full recovery)

or

D. LOST (Phase offset remaining) MICROSECONDS, (Amplitude)

or

D. HOUR (Phase offset remaining) MICROSECONDS, (Amplitude)

or

D. (Phase offset remaining) MICROSECONDS, (Amplitude), SECOND OIISET 
where: Time from onset is three digits of tenths of minutes. Amplitude is given in $\mathrm{dB}$.

Phase offset remaining is the phase difference between the current phase value and that needed for full recovery.

\section{LINE 8: (Paragraph E)}

E. (Tracking Rate), (Sigma at Onset) MICROSECONDS, (Type)

where: Tracking Rate is one digit value of sensor setting

Sigma is the onset standard deviation value

Type is repeated from Line 2, above.

\subsection{Input Parameter Descriptions}

\section{PARAMETER PROGRAM DESCRIPTION}

E.BDF $\quad E \$ B D C \quad$ Buffer of decimal digits from binary to decimal conversion.

U.FRQ E\$LOG ASCII characters of sensor frequency settings.

U.TRK U\$DAT Current setting for sensor channel tracking rate.

U.GAN U\$DAT Current setting for sensor channel RF gain.

U.BLL U\$DAT Current setting for sensor blanking level.

M.STA MSESC Digital IOIS status word from sensor channel.

U.CLS USDAT Waveform class corresponding to criteria met.

U.ODR USDAT Direction of onset; $=1$ for advance; $=2$ for retard; $=3$ for multiple peak advance.

U.OSG U\$DAT Standard deviation (U.SIG) at onset.

$\left.\begin{array}{lll}\text { E.TM1 } \\ \text { E.TM2 }\end{array}\right\}$ E\$FRT $\quad$ Two word BCD time used as parameter vat

U.AMP U\$DAT Current signal amplitude estimate. 


\subsection{Input Parameter Descriptions}

\section{PARAMETER PROGRAM DESCRIPTION}

U.OTB USDAT Signal onset time, BCD format.

U.OFZ USDAT Phase value at onset.

U.OAM U\$DAT Signal amplitude at onset.

U.ODI U\$DAT Long term phase trend estimate at onset (U.DIU).

W.PFZ U\$DAT Phase vaiue at peak.

W.PAM U\$DAT Signaī amplitude at peak.

W.RTM U\$DAT BCD time of valid recovery.

W.RFZ U\$DAT Phase value at valid recovery.

W.RAM U\$DAT Signāl amplitude at valid recovery.

U.DIU USDAT Current long-term phase trend (diurnal slope) estimate.

W.LTM U\$DAT BCD time at first peak of multipie peak signai.

W.LPK USDAT Phase value at first peak of multiple peak signal.

W.LAM USDAT Signal amplitude at first peak of multiple peak signal.

W.VTM USDAT BCD time at valley peak of multiple peak signal.

W.VPK U\$DAT Phase value at valley peak of multiple peak signal.

W.VAM USDAT Signal amplitude at valley peak of multiple peak signal.

W.OST U\$DAT Phase offset, onset to peak.

W.ONR USDAT Average rate of onset, onset to peak.

U.LFZ U\$WFA Phase value of the last compressed data point encountered on each channel.

U.LTM U\$WFA The BCD character time of the last compressed data point encountered on each channel. 


\begin{tabular}{|c|c|c|}
\hline PARAMETER & PROGRAM & DESCRIPTION \\
\hline U.END & U\$DAT & $\begin{array}{l}\text { Flag to indicate how processing terminated on } \\
\text { waveform just completed. }\end{array}$ \\
\hline W.PTM & U\$DAT & BCD time at peak. \\
\hline U.DTM & U\$DAT & Dummy binary time used by E\$DIF. \\
\hline U.OTM & USDAT & Signal onset time, binary format. \\
\hline U.CTM & U\$DAT & BCD time of current ten-second interval. \\
\hline E.BSG & $E \$ B D C$ & $\begin{array}{l}\text { ASCII characters for sign of output of } E: B D C \text {, } \\
\text { binary to decimal conversion. }\end{array}$ \\
\hline U.CCL & U\$WFA & $\begin{array}{l}\text { Number of microseconds in one cycle for the } \\
\text { frequencies of each channel. }\end{array}$ \\
\hline
\end{tabular}

17.5 Output Parameter Descriptions

PARAMETER SIZE DESCRIPTION

$U: R P 1 \quad$ Entry point to routine to generate Part I of RSD.

U:RP2 Entry point to routine to generate Part II of RSD.

U:RP3 Entry point to routine to generate Part II I of RSD.

U:UDR $\quad 1 \quad$ Serial counter for reportable signals.

\subsection{Internal Data Descriptions}

PARAMETER SIZE DESCRIPTION

MSGD1 28 Part I \& II of RSD, Line 1
MOISI 
17.6 Internal Data Descriptions (continued)

PARAMETER SIZE DESCRIPTION

MO1S2

$\mathrm{M} 01 \mathrm{S3}$

M91S4

$\mathrm{M} 9155$

MSG92

M92ST

Mø252

MSG $\$ 3$

MO3ST

Mø3S2

MSGQ4

M94S 1

LEADS

LEADR

MSG 95

Ma5S1

MSGQ6

Mø6ST 1

CALTR

LLABE

UDRNO

LINEN

TYPEA

TYPEB

TYPEC
62

22

20

19

3

27

23

82

56

6

List of. data line Tabels; "ON", "P1", "P2", "PK", "HR", and "FR".

14 Value of serial counter for signal on each channel.

Pointer to current line number; entry in LLABE.

3 "ALPHA" in ASCII characters.

3 "BRAVO" in ASCII characters.

3
Part I \& II of RSD, Lines 2 through 5.

Part I \& II of RSD, buffer for lines of numeric data.
Part II of RSD, Line 7.

$C / R$ followed by five $L / F$ characters.

Buffer of null characters for leader generation.

Buffer for Part III of RSD, Line 1.

Buffer for Part III of RSD, Lines 2 through 8.

Table of frequencies and associated call letters.

"ALPHA" in ASCII characters.

"COCO" in ASCII characters. 


\subsection{Internal Data Description (continued)}

\begin{tabular}{|c|c|c|}
\hline PARAMETER & SIZE & DESCRIPTION \\
\hline TYPED & 3 & "DELTA" in ASCII characters. \\
\hline TEMP & 5 & Working storage. \\
\hline TPHAS & 1 & Parameter for internal subroutine; phase value. \\
\hline TAMP & 1 & Parameter for internal subroutine; amplitude value. \\
\hline TOIUR & 1 & Parameter for internal subroutine; diurnal slope estimate. \\
\hline RPSVI & 7 & Register save area. \\
\hline RPSV2 & 6 & Register save area. \\
\hline END1 & 6 & "NORMALLY" in ASCII characters. \\
\hline END2 & 6 & "TIME LAPSE" in ASCII characters. \\
\hline END3 & 6 & "SIGNAL LOST" in ASCII characters. \\
\hline END4 & 6 & "NEW ONSET" in ASCII characters. \\
\hline NOTBL & 24 & Pointers to ASCII characters of words in output messages. \\
\hline NOØ & 3 & "ZERO" in ASCII characters. \\
\hline N01 & 3 & "ONE" in ASCII characters. \\
\hline NO2 & 3 & "TWO" in ASCII characters. \\
\hline N03 & 4 & "THREE" in ASCII characters. \\
\hline N04 & 3 & "FOUR" in ASCII characters. \\
\hline N05 & 3 & "FIVE" in ASCII characters. \\
\hline N06 & 3 & "SIX" in ASCII characters. \\
\hline N07 & 4 & "SEVEN" in ASCII characters. \\
\hline N08 & 4 & "EIGHT" in ASCII characters. \\
\hline N09 & 4 & "NINE" in ASCII characters. \\
\hline
\end{tabular}


17.6 Internal Data Descriotion (continued)

PARAMETER SIZE DESCRIPTION

N010

N011

N012

N013

N014

N015

N016

N017

N018

N019

N029

N021

N022

N023

ZCLS

ZTYPE

ZFRQ

CALLT

ZSTA

ZOTM

ZOTB

ZOAM
4

3

3

3

3

3

3

7

5

4

3

4

2

7

14

14

28

28

14

28

28

14
"FRONT" in ASCII characters.

"BACK" in ASCII characters.

"PEAK" in ASCII characters.

"HALF" in ASCII characters.

"FULL" in ASCII characters.

"HOUR" in ASCII characters.

"LOST" in ASCII characters.

"MICROSECONDS" in ASCII characters.

"ADVANCE" in ASCII characters.

"RETARD" in ASCII characters.

"PLUS" in ASCII characters.

"MINUS" in ASCII characters.

"DB" in ASCII characters.

"SECOND ONSET" in ASCII characters.

Copy of U.CLS

Pointer to TYPEA, TYPEB, TYPEC, or TYPED above.

Copy of U.FRQ

Computed call letters for sensor frequencies.

Copy of M.STA

Copy of U.OTM

Copy of U.OTB

Copy of U.OAM 
17.6 Internal Data Description (continued)

\begin{tabular}{|c|c|c|}
\hline PARAMETER & SIZE & DESCRIPTION \\
\hline ZOST & 14 & Copy of W.OST \\
\hline ZODR & 14 & Copy of U.ODR \\
\hline ZONR & 14 & Copy of W.ONR \\
\hline ZPTM & 28 & Copy of W.PTM \\
\hline ZPAM & 14 & Copy of W.PAM \\
\hline ZRTM & 28 & Copy of W.RTM \\
\hline ZRAM & 14 & Copy of W.RAM \\
\hline ZRFZ & 14 & Copy of W.RFZ \\
\hline ZTR & 14 & Copy of U.TRK \\
\hline ZOSG & 14 & Copy of U.OSG \\
\hline ZOFZ & 14 & Copy of U.OFZ \\
\hline ZFAM & 14 & Copy of U.AMP \\
\hline ZFFZ & 14 & Copy of U.LFZ \\
\hline ZFTM & 28 & Copy of U.LTM \\
\hline INPRG & 14 & Flag to indicate on which channels a report is in progress \\
\hline ZEND & 14 & Copy of U.END \\
\hline
\end{tabular}



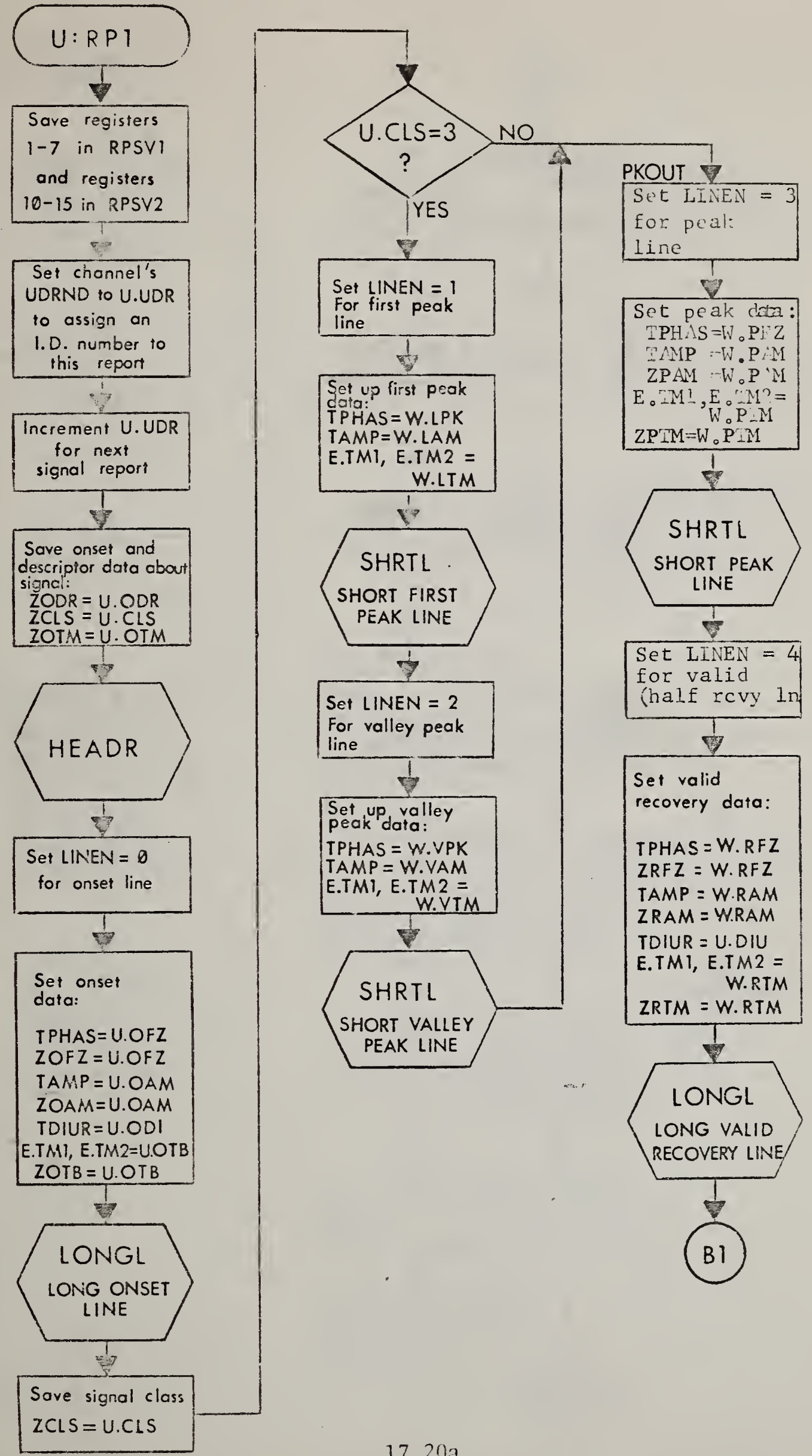


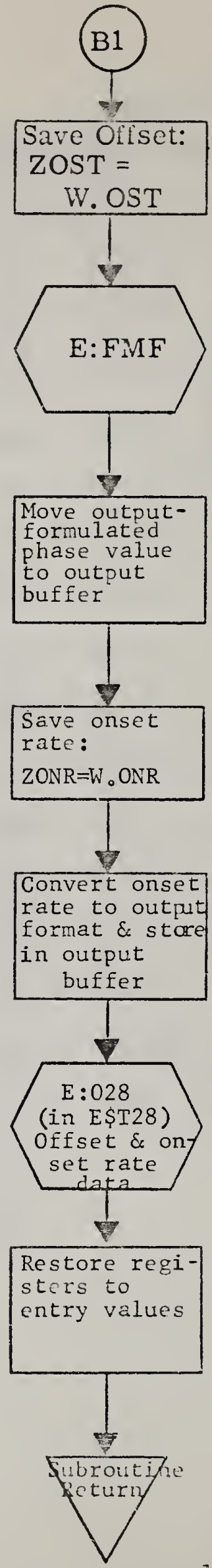




\section{U:RP2}
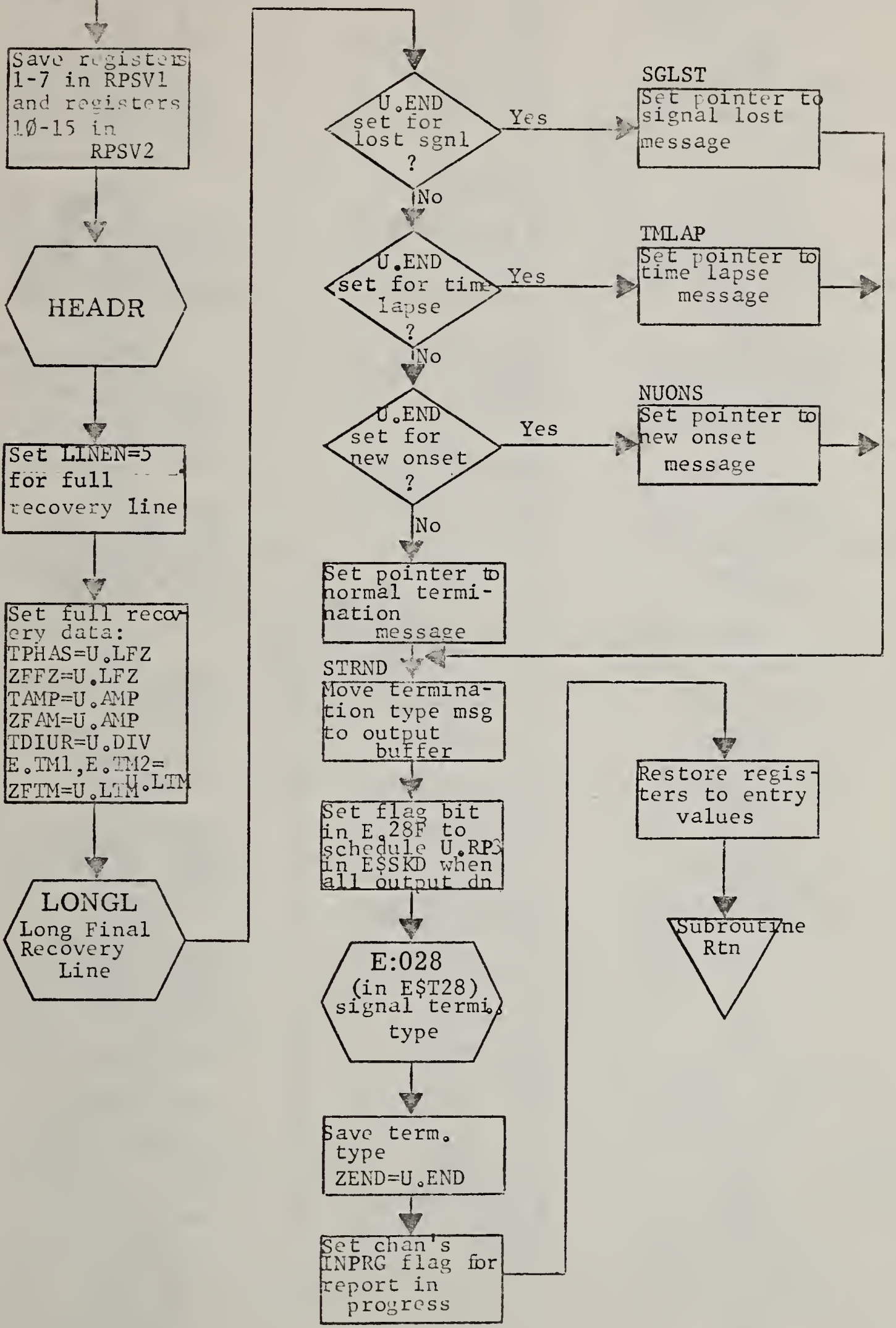


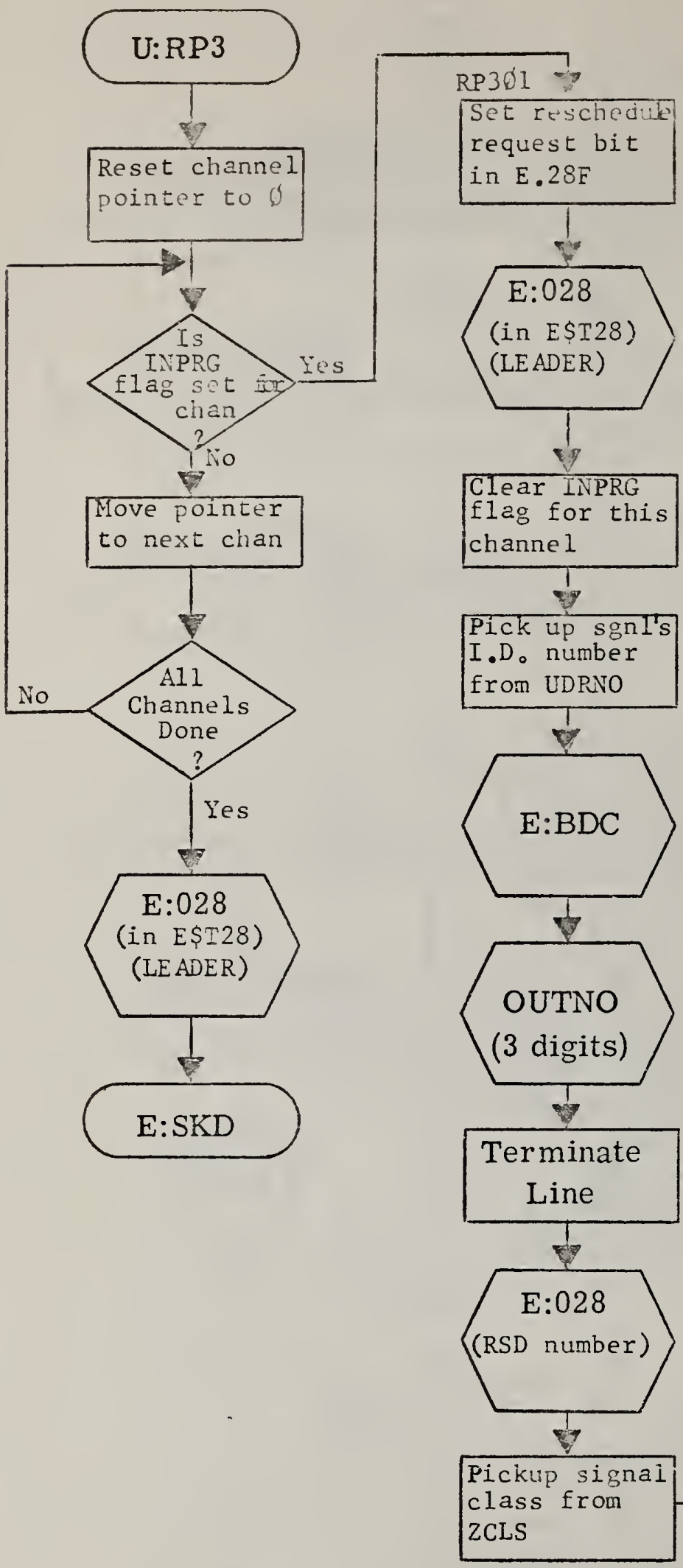

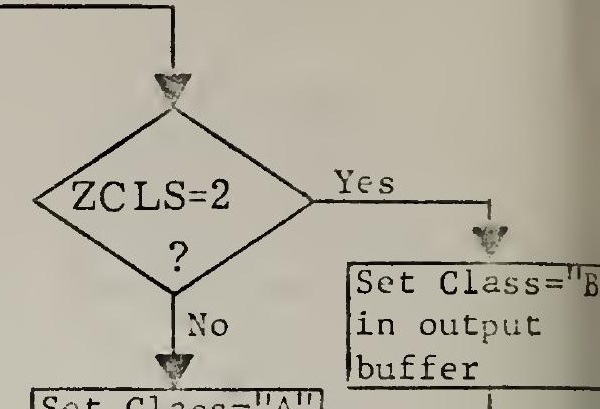

Set Class="A" in output

buffer

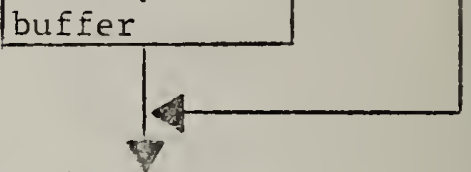

Store comma (非2C) in output buffer

Set pntr to sig type (Adt. of alphabetic chars of type chars of ty

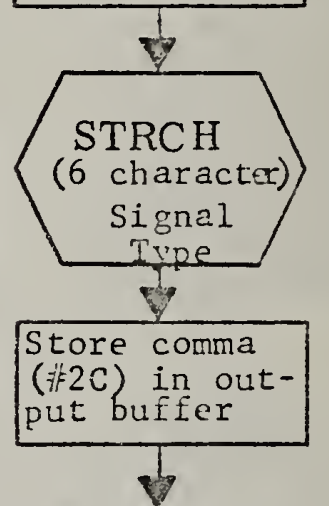

Set pntr to charac of channel's freq from

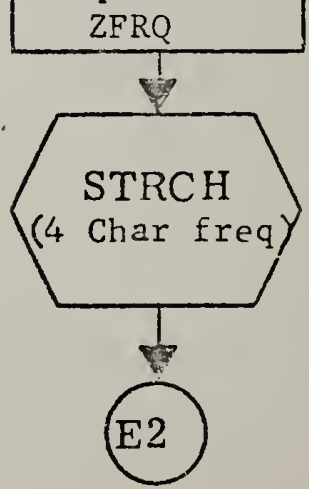




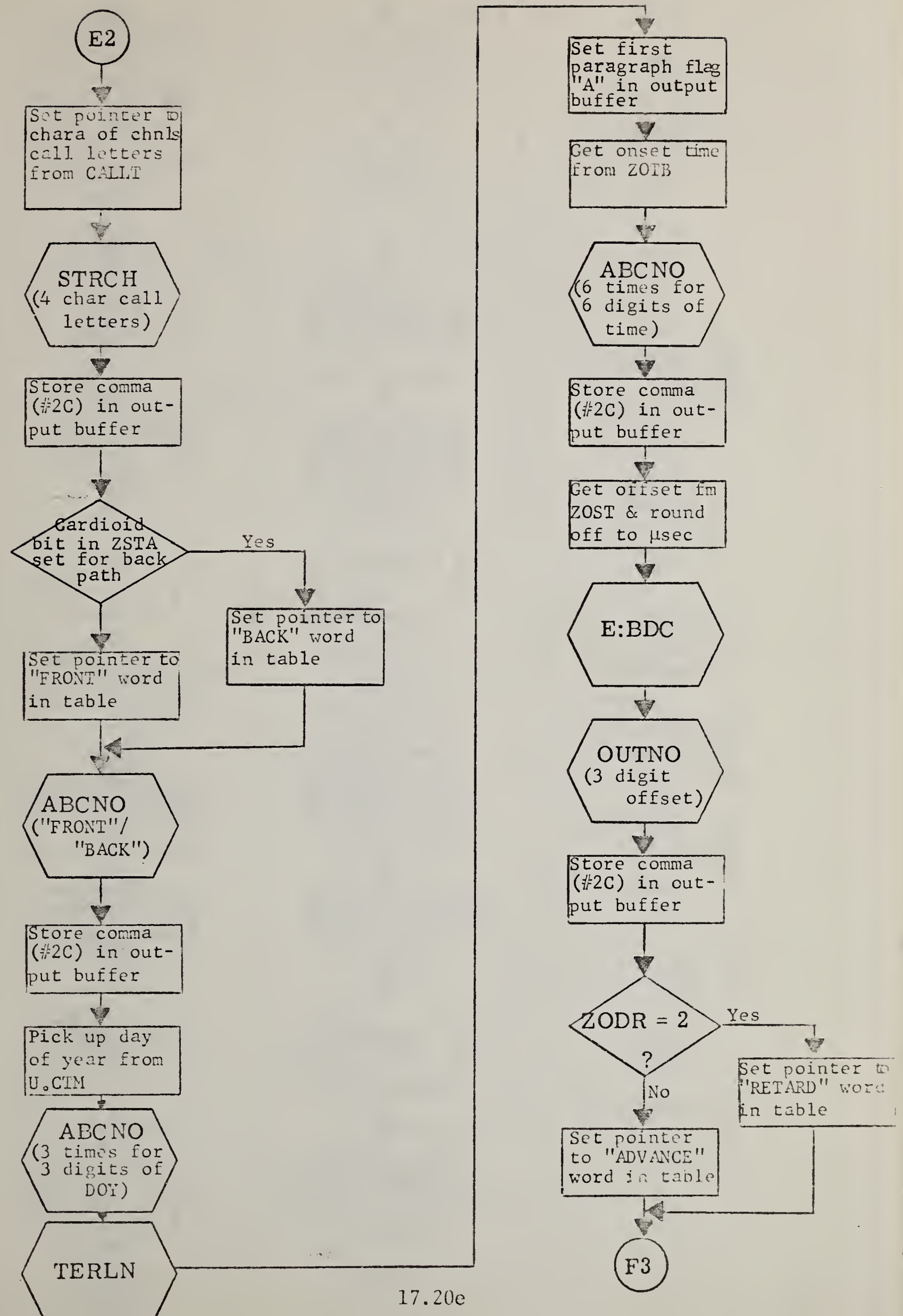



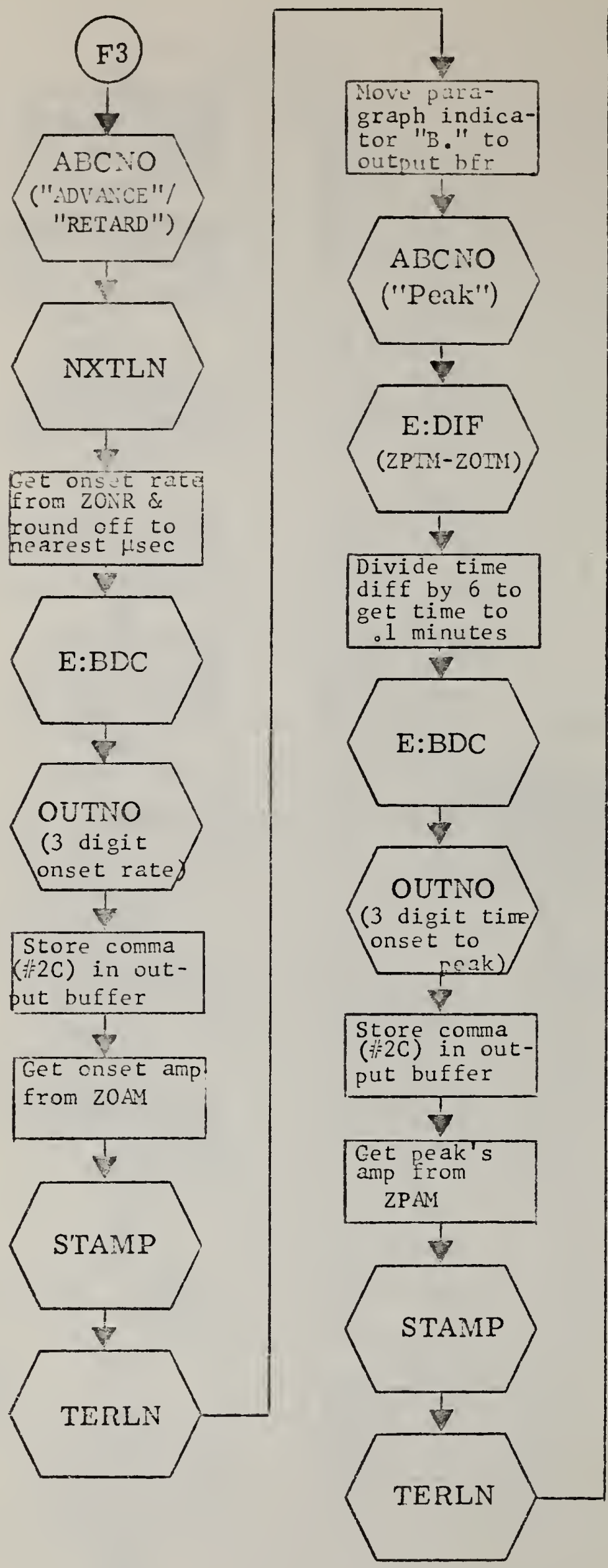


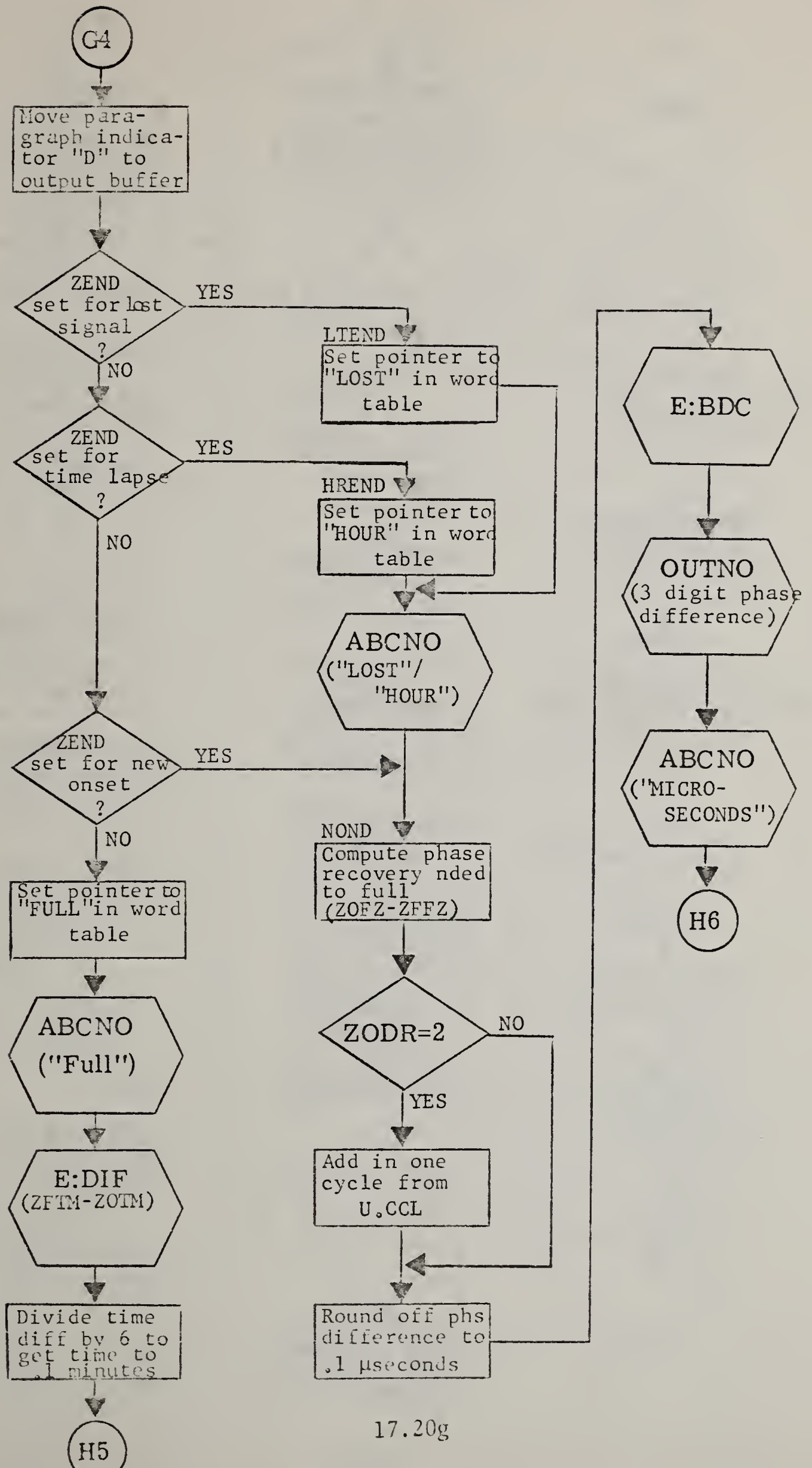




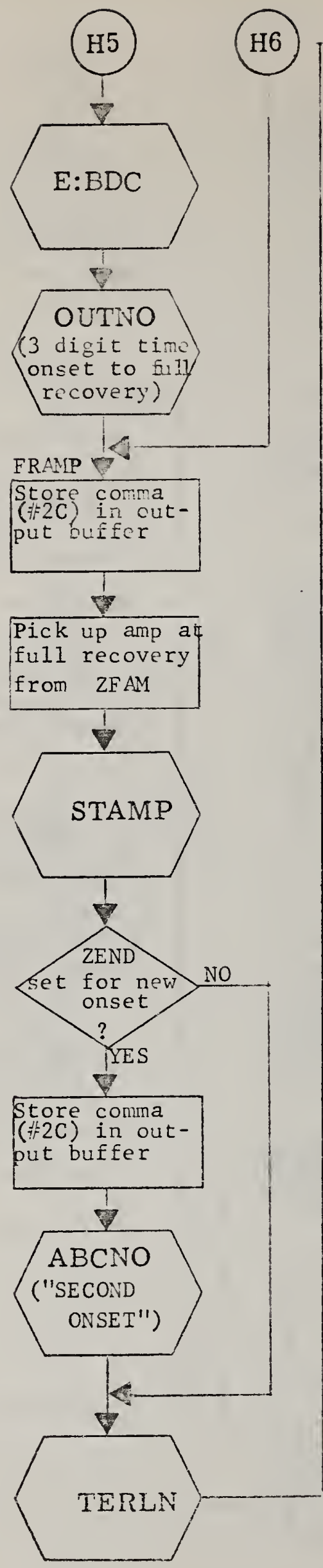

Move para-

graph indica-

tor "E"。 to

output buffer

Pick up chan's tracking rate

TRR

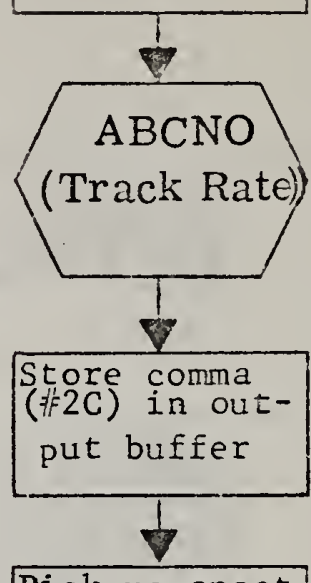

Pick up onset sigma from ZOSG and scale to give. .1 microseconds

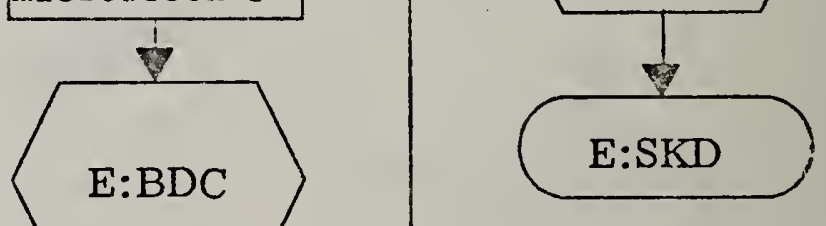

STRCH

(6 characters

of signal

type)

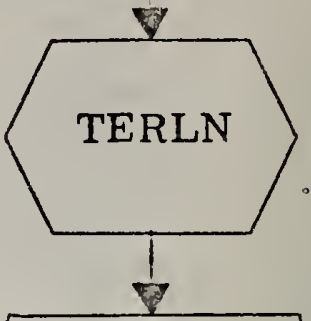

Set flag bit in $E_{0} 28 \mathrm{~F}$ to reschedule
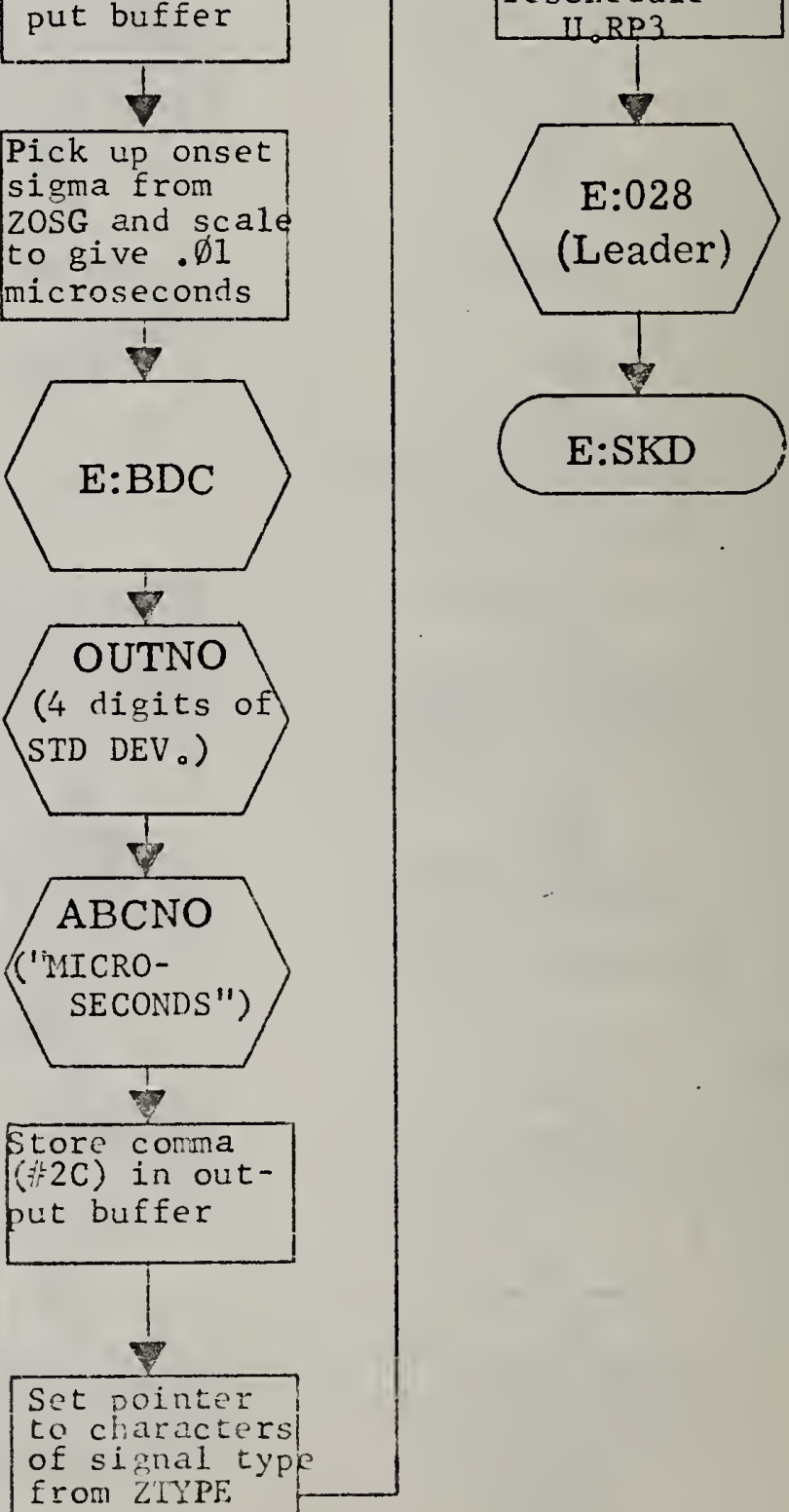


\section{HEADR}

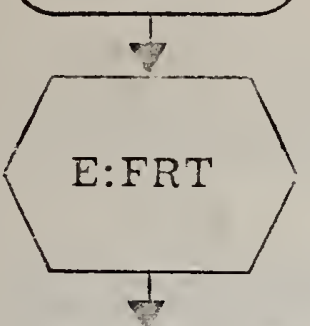

Read det

no. from

store in

output buffer

$r$

Pick US signal ID no. from UDRND

\%

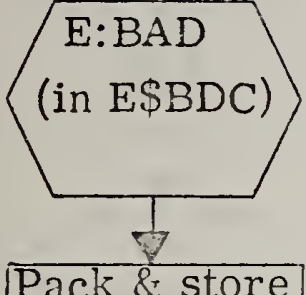

ID no. in output buffer

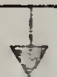

Convert chn

designator \& store in output buifer $\rightarrow$

Get chint's

freg. from

U. FRQ \& SV

in ZFRQ

HED $\varnothing 2$

No

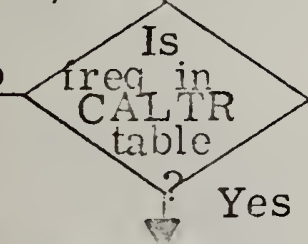

Get callitrs

for in irea.

fm table esar

in CALL'T

HED
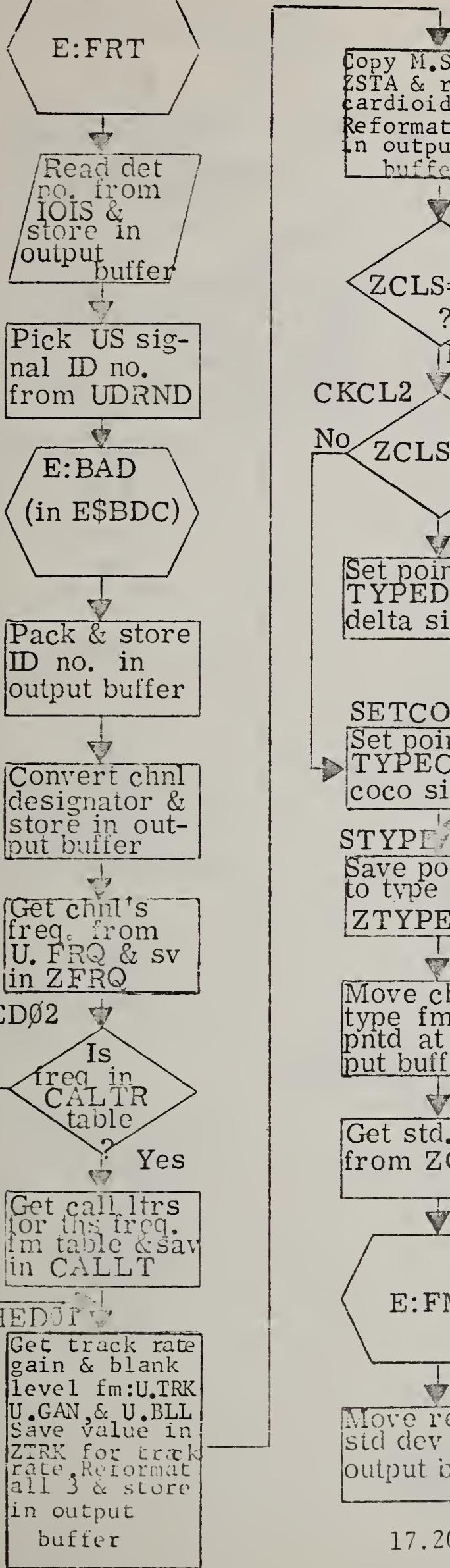

Copy M.STA into

STA \& recover

cardioid config

Reformat \& str

in output

buef

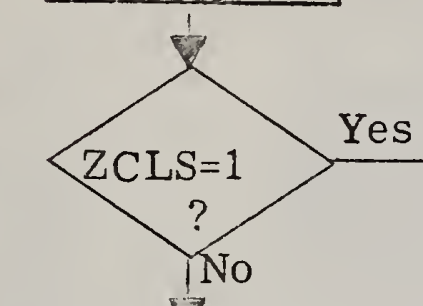

CKCL2

$\mathrm{ZODR}=1$
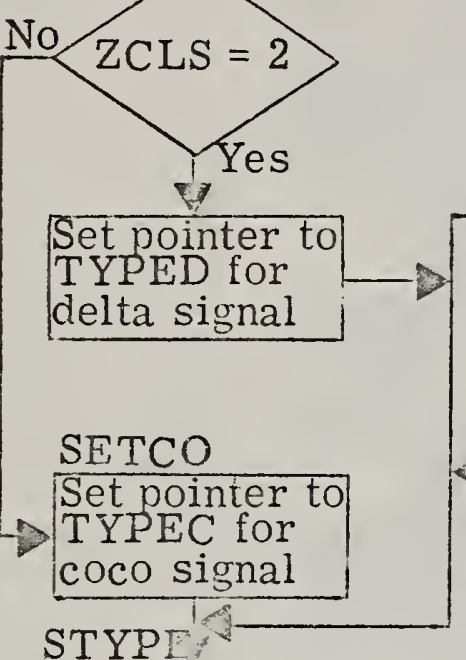

Set pointer to TYPEA for alpha signal

Save pointer

to type in

ZTYPE

Move chars of

type fm lctn pntd at to output buffer

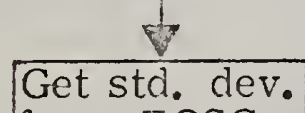
from ZOSG

SETBR

Set pointer to TYPEB for bravo signal

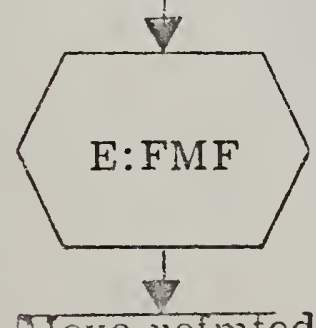

More refmted

std dev to output buffer

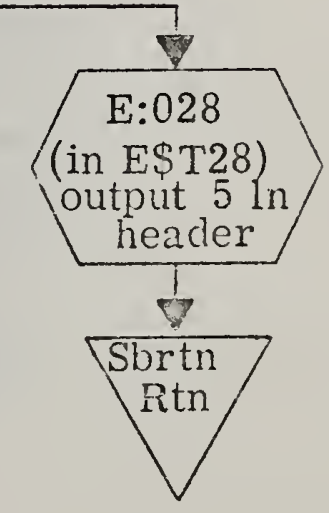




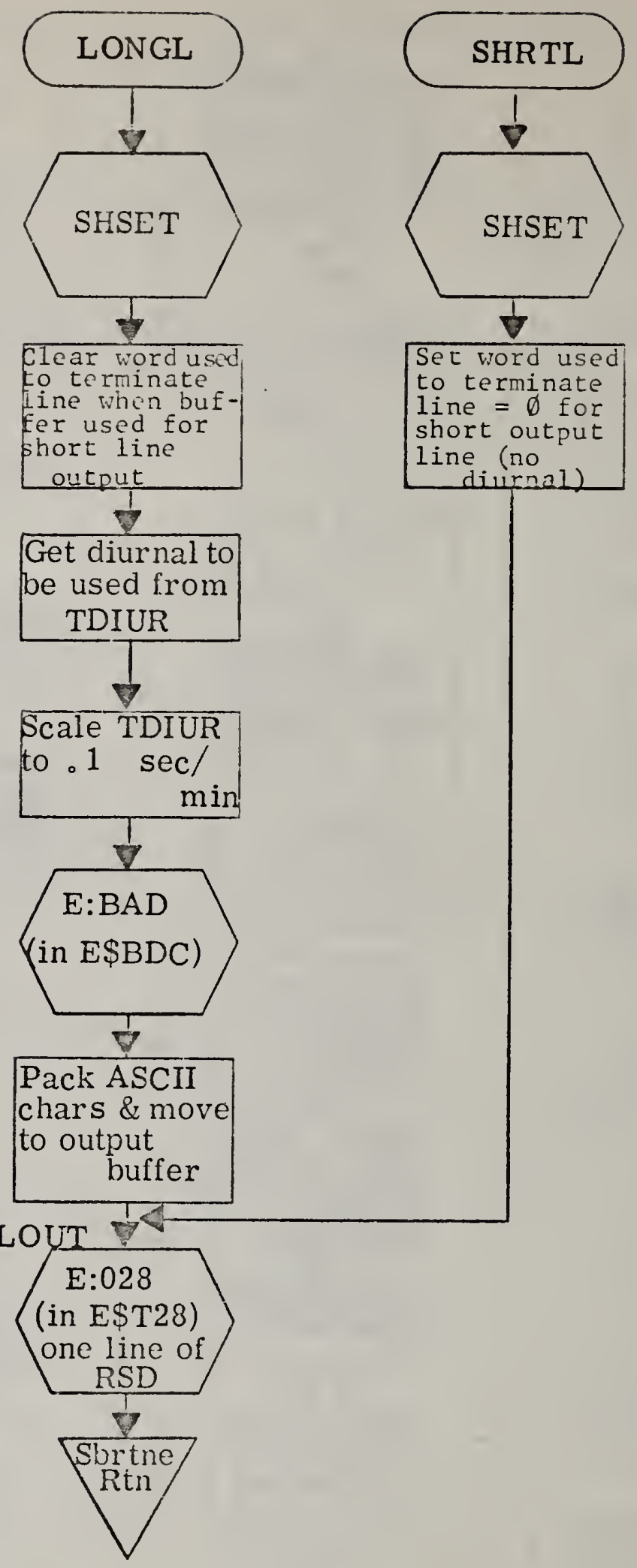




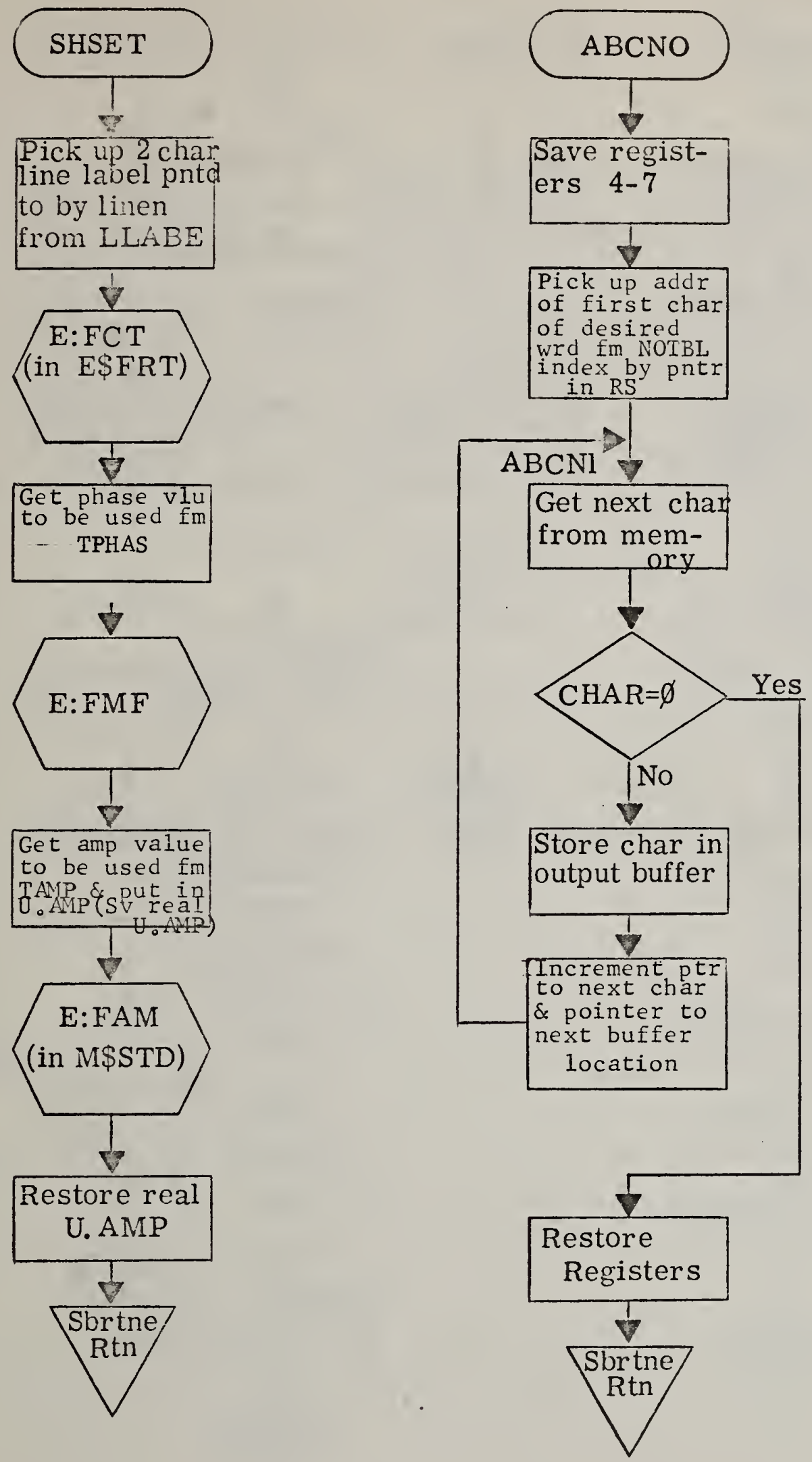




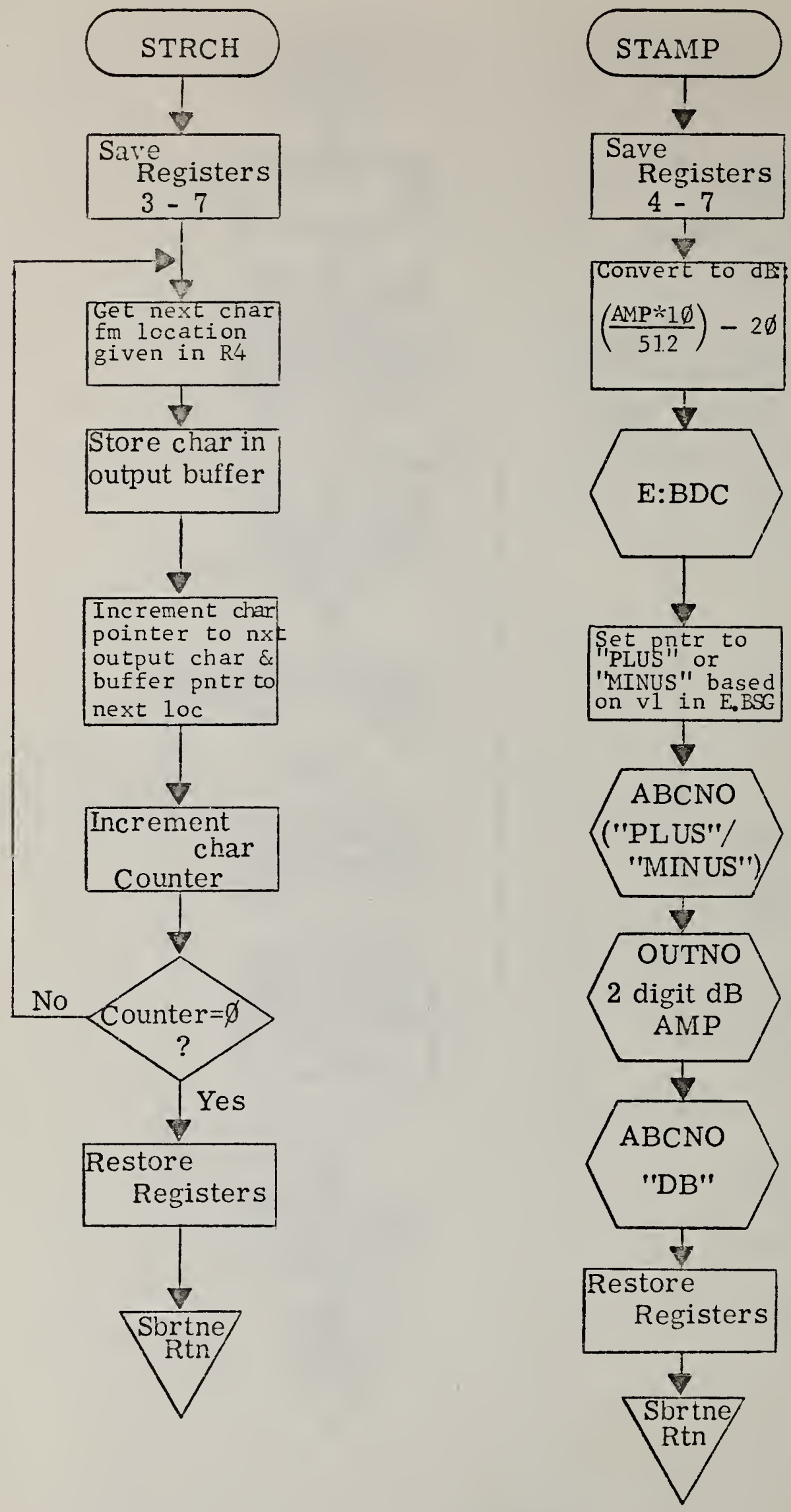




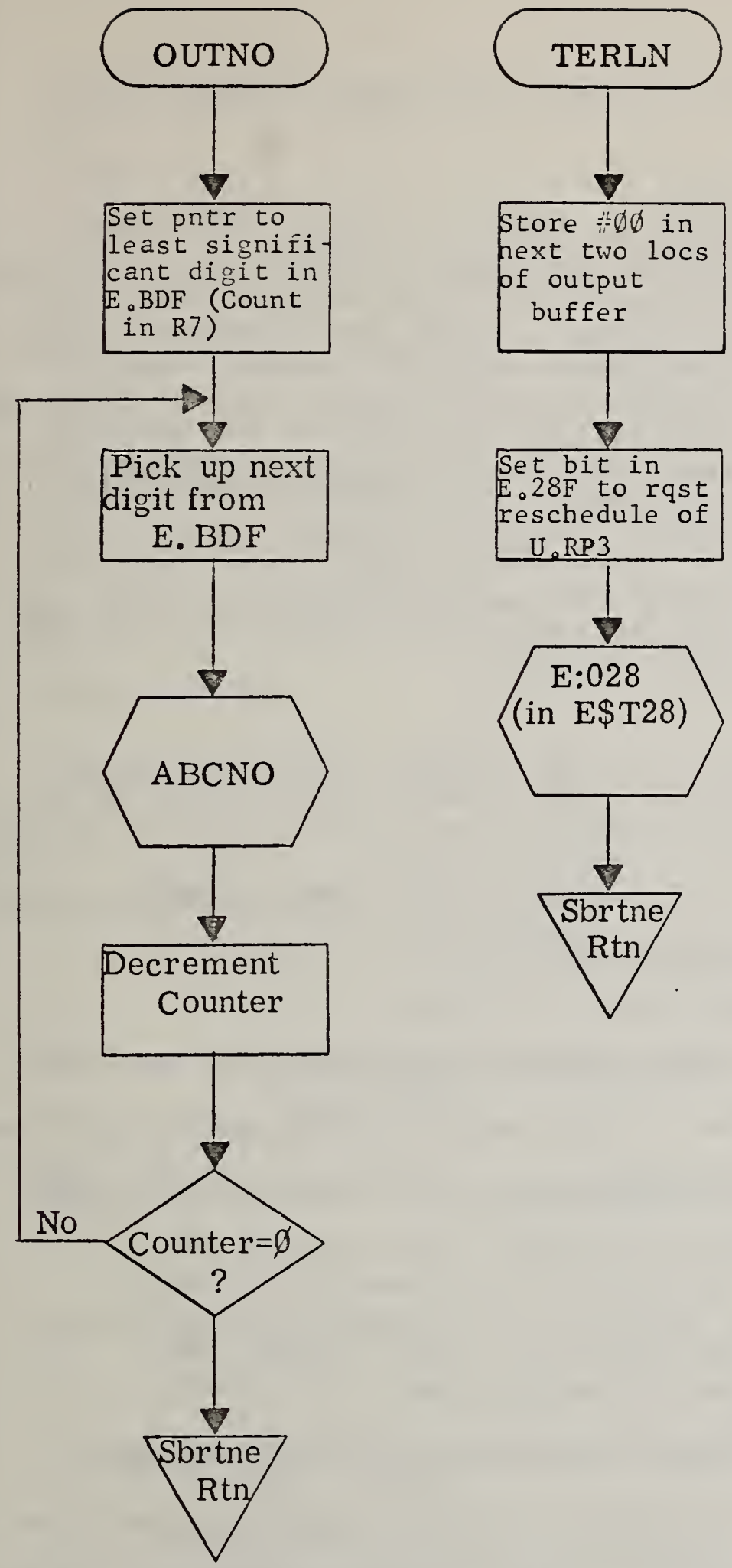

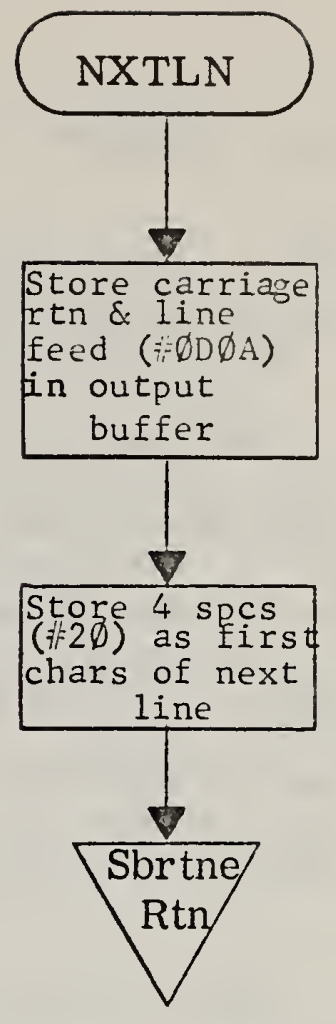

\section{NXTLN}

riage

feed ( $\varnothing D \emptyset \mathrm{D}$ )

in output 
18. U\$RED

\subsection{Functional Description}

As NUSTAD is designed to run unattended for extended periods of time, and the system mission requires that reliable data be maintained on at least the highest priority channels, several sensor channels may be tuned to the same frequency to supply back-up coverage of the high priority paths. U\$RED determines whether those sensor channels tuned to the same frequencies are arriving at the same processing results and logs those cases where channels fail to do so.

U\$RED is entered at U:RED from the scheduler. This task may be scheduled either by E:MIN at a regular interval, or by $E: O M I$ in response to an operator request (the CRC: input option). When completed, control is returned to the system scheduler at E:SKD.

U:RED first pairs ali sensor channels which monitor the same paths. Where three channels monitor the same path, the three possible pairs are al1 detected. Two channels are considered to be monitoring the same path if:

a. Neither sensor channel has its processing inhibited; i.e., neither has a Maintenance or INE condition set.

b. Both sensor channels are tuned to the same frequency.

c. If both sensor channels are using a cardioid pattern, the FRONT/BACK directional selection is the same for both. If both are not 
using a cardioid pattern, they will be considered to be in the same direction.

When a pair of sensor channels are encountered monitoring the same path, the processing results for those channels will be compared. If the resuits compare favorably, the channel pair will have passed the redundacy test successfully. If all pairs test successfully, a "T RS" (Total system Redundancy test Successfu1) message is entered into the station log. Where some pair fails, a message is generated indicating the sensor channels add the reason for the failure. The format for that message is:

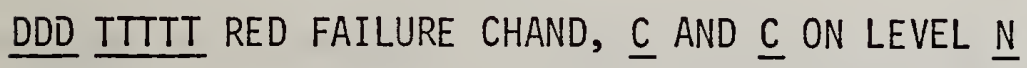

where: $\underline{D}$ is a digit of DOY

$T$ is a digit of TOD

$\underline{C}$ is a character of sensor channel designation ( $A$ through $N$ )

$\underline{N}$ is a digit indicating where the test failed, interpreted as follows:

LEVEL REASON

1 The channel status, determined by the amplitude characteristics, is not the same for both channels. (U.CST)

2 The anatysis status, indicating the progress in waveform processing, is not the same for both channels. (U.AST)

3 The short term noise characteristics are not the same on both channels. (U.TRK)

4 The long term trend characteristics are not the same on both channels. (U.DIU) 
If no pair of channels monitoring the same path are encountered, no message is generated.

The tests conducted on the channel pairs include:

a. Channel Status. The pair passes this test only if both values of U.CST are the same.

b. Analysis Status. The pair passes this test only if both values of U.AST are the same.

c. Short Term Noise Characteristics. Since the tracking rate is adjusted on the basis of the signal standard deviation, the use of the tracking rate settings as a basis for this test is deemed reasonable. Since monir variations in the sensor itself can produce slight differences, identical settings will not be required. Therefore, the pair will pass this test if the tracking rates (U.TRK) for the two channels are within three settings of one another.

d. Long Term Trend Characteristics. Again, minor variations in the sensors will cause some differences in these estimates. The pair will pass this test if the diurnal slope estimates (U.DIU) are within .1915625 microseconds/minute of one another.

\subsection{Called Subroutines}

SUBROUTINE PROGRAM FUNCTION

E:SKD E\$SKD System scheduler reentry point.

E:028 E\$T28 Translates output message to BAUDOT code and stores in output buffer before transmission to ASR 28.

E:LOG E $\quad$ \$LOG Generates coded log message for ASR 28.

E:FRT E\$FRT Reformats BCD time from the WTC to ACCII for output. 
18.3 Input Parameter Descriptions

PARAMETER PROGRAM DESCRIPTION

$\begin{array}{ll}\text { U.CST U\$DAT } & \text { The channel's processing status, based on the } \\ \text { results of the amplitude monitoring section of } \\ \text { U:RTA. }\end{array}$

U.AST U\$DAT The channel's waveform analysis status, determined by the point in the signal to which processing has progressed.
U.TRK U\$DAT Current settings for sensor channel tracking rates.
U.DIU U\$DAT The diurnat slope, or long term data trend, estimate.
E.28F E\$T28 Parameter word used in calling E:028.
U.FRQ E\$LOG The current settings of the sensor frequency switches.
U.IHB U\$DAT Flag bit set to inhibit processing on a channel when a Maintenance or INE condition exists.
M.STA M\$ESC IOIS digital input word with frequency, INE, Maintenance, Auto/Manual, and Cardioid settings for each channel.

18.4 Output Parameter Descriptions

PARAMETER SIZE DESCRIPTION

U:RED Entry point for channel redundancy checking routine.

18.5 Internal Data Descriptions

PARAMETER SIZE DESCRIPTION

FMSG $27 \quad$ "123 123456 RED FAILURE, CHANS A AND B ON LEVEL 7" message text.

FLAG $\quad 1 \quad F l a g$ word for status of U:RED processing:

Bit $\emptyset=1$ if faitures have been found.

Bit $1=1$ if sensor channel pairs monitoring same path found. 


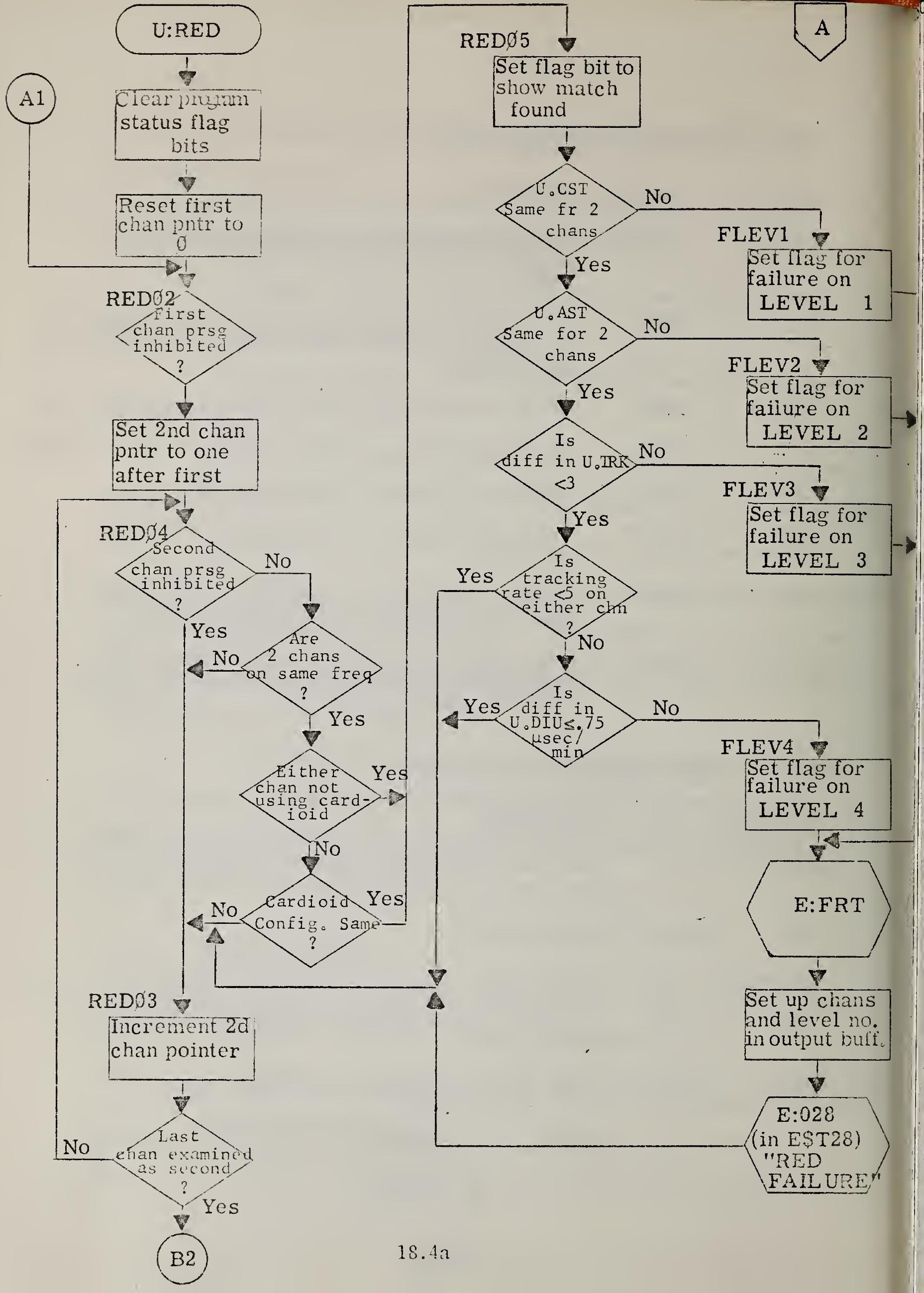


19. U\$PST

19.1

Functional Description

This program allows the operator to make changes to the sensor tracking rate, gain, and blanking level by typing the desired value with a keyword identifier and sensor channel information on the ASR 35 teletype. NUSTAD responds by either carrying out the desired task or explaining why it couldn't.

\subsubsection{U:PST}

U:PST is entered from the system scheduler when E:OMI identifies an operator keyword designation of OPC: and serves two primary functions:

a. A Tink between NUSTAD and the operator used to pass parameter values to the sensor channels.

b. Text editing to prevent improper parameter values from being transmitted.

Parameter change messages are fixed format as explained in Section 19.3 and are passed via the ASR 35 and NUSTAD to the sensor channels. Prior to any change being made, a check (MAYI) is made on the affected channel to determine if a signal is in progress. If a signal is in progress, a message is output explaining that no change can be made at that time. Once a change is made, control is returned to NUSTAD and the operator must reinitiate the calling sequence to make additional changes. 
19.2 Called Subroutines

SUBROUTINE PROGRAM FUNCTION

E:035 E\$T35 Transfer output message to buffer before transmission to the ASR 35 .

U:CNI U\$RTA Entry point for routine to determine whether a signal is in progress for granting permission to make sensor parameter setting adjustments (see Section 12.1.2).

E:SKD E\$SKD System scheduler entry point.

E:MIT E\$UIN Transmits GAIN, TRACKING RATE, and BLANKING LEVEL values to sensor channel given in R3.

E:LOG E\$LOG Generate coded log message for ASR 28.

19.3 Data Formats

The operator calling sequence used in this program includes the keyword OPC: followed by the channel designation, function code, and parameter values desired. The channel designations are the letters $A, B, C$, through $N$, and correspond to the fourteen sensor channels. A single letter is used to denote the function desired, B for BLANKING LEVEL change, G for GAIN chainge, and T for TRACKING RATE change. Each of these functions have an upper and a lower limit to the value that can be requested; BLANKING level ranges from $\emptyset \emptyset$ through 63 , GAIN from $\emptyset \emptyset$ through 24, and TRACKING RATE from $\emptyset \emptyset$ through $\emptyset 9$. The request should take the form of the following example: 


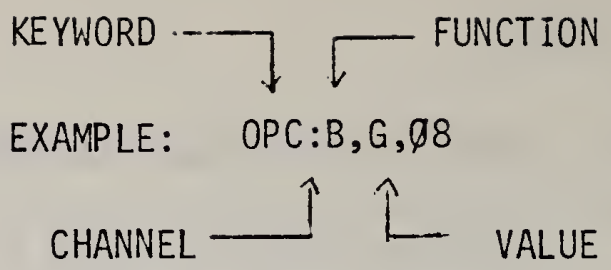

The request should be followed by a carriage return.

19.4 Input Parameter Descriptions

PARAMETER PROGRAM DESCRIPTION

U.GAN U\$DAT Current sensor RF GAIN setting (see Chapter 16).

U.TRK U\$DAT Current sensor TRACK RATE setting (see Chapter 16).

U.BLL U\$DAT Current sensor BLANKING LEVEL setting (see Chapter 16).

E.35I E\$T35 Address of first character of input from ASR 35 .

E.IOF E $\$ T 35$ Parameter word used in calling E\$T35.

19.5 Internal Data Description

PARAMETER SIZE DESCRIPTION

ILLCH 11 "INVALID CHANNEL" output message.

ILLVL $15 \quad$ "VALUE NOT WITHIN LIMITS" output message.

NOPAR 23 "NO PARM. CHANGE PERMITTED, SIG. IN PROG." output message.

ILFUN $\quad 11$ "INVALID FUNCTION" output message.

INFMT $\quad 1 \varnothing \quad$ "INVALID FORMAT" output message.

ILVAL 14 "CANNOT USE NEG. VALUES" outplit message. 


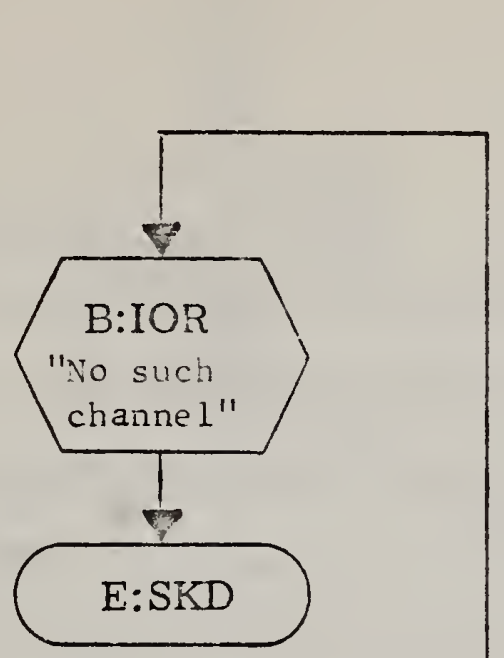

\section{USPST}

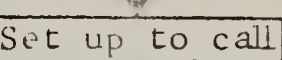
B:IUR with

following

message

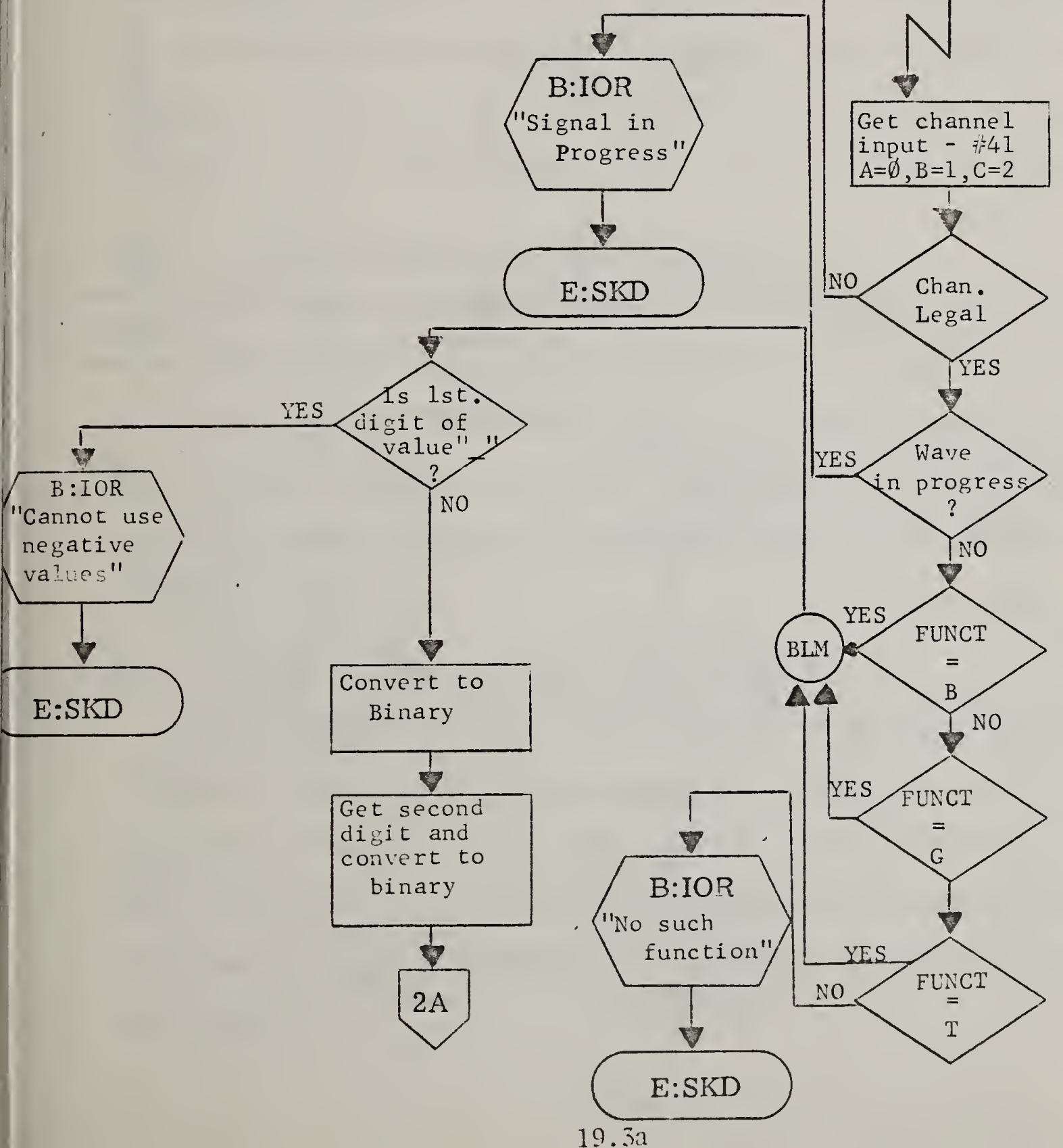




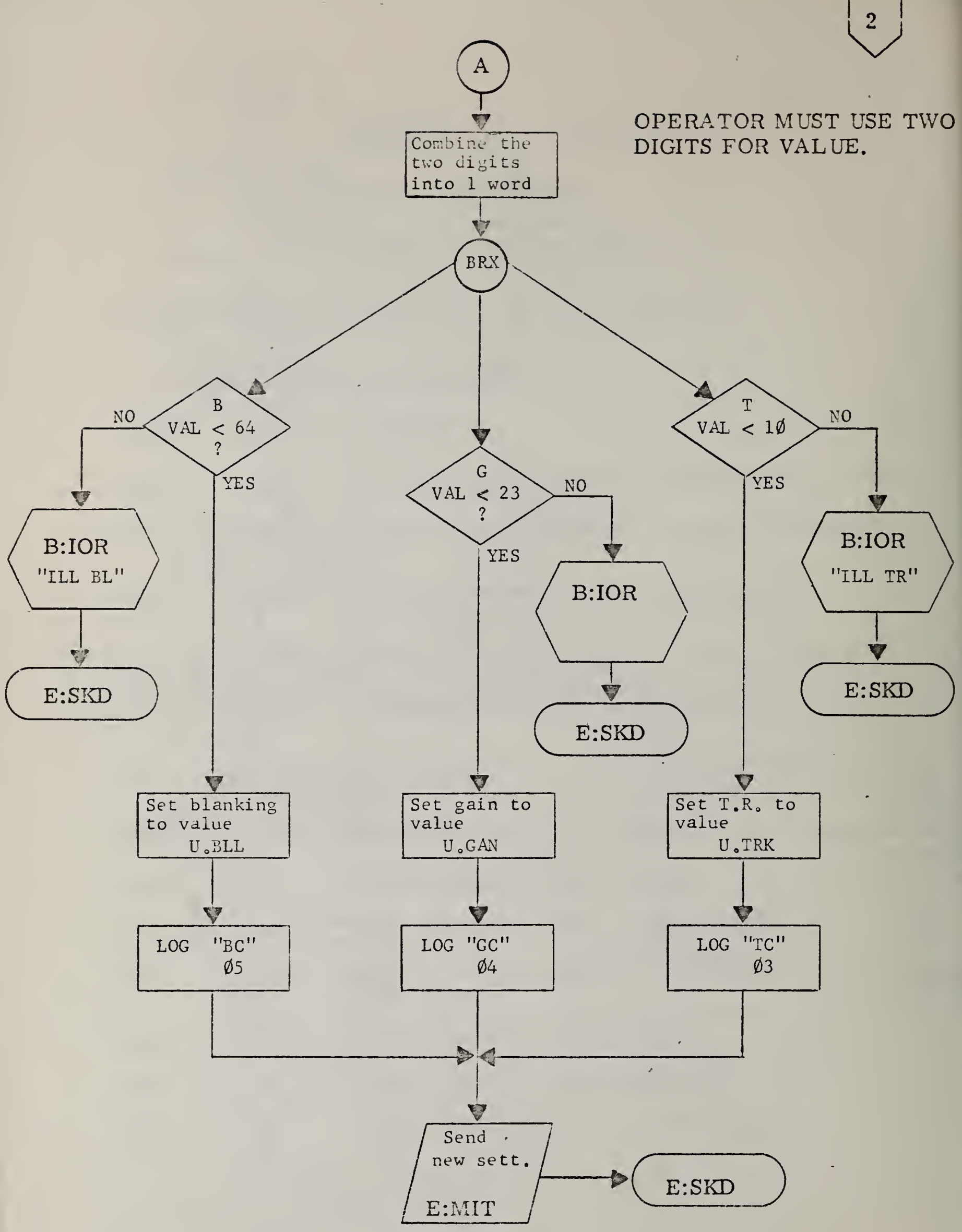


20. M\$ESC

$20.1 \quad$ Functional Description

NUSTAD monitors the hardware status of the sensor and ADACS interface through digital information transmitted from these devices to the processor via the IOIS digital input. Specifically, the sensor frequency setting, the cardioid configuration, the position of the maintenance and auto/manual switches, and the status of the INE check are made available to the system through fourteen words, one per sensor channel, of the digital input portion of the IOIS. When any of these values change, the level three user defined external interrupt of the IOIS, referred to as the INE interrupt, vectors control to M:INT of M\$ESC.

\subsubsection{M:INT (INE Interrupt Service Routine)}

An INE interrupt vectors control to M:INT when any of the information in the digital status words in the first fourteen channels of the digital IOIS changes. M:INT then sets a five second delay timer (M.TMI) in $E: S E C$ when the timer reaches zero, E:SEC flags M:ESC in the system scheduler. After starting the delay timer, M:INT returns control to the interrupted program.

\subsubsection{M:ESC (Equipment Status Change Monitor).}

M:ESC is entered from the system scheduler to determine the cause of the INE interrupt and to react accordingly. The previous values for the digital status words are stored in M.STA (known internally as SVWRD). Channel by channel, the current values are read and compared with the old values. The following actions are taken when the various parameters have changed: 
a. Auto/Manual bit changed. An "AU" message is generated for the station log if the change is to the Automatic position, or a "MN" message if the change is to the Manual position.

b. Maintenance bit changed. A "MS" or "MR" message is generated for the station $\log$ to indicate whether the maintenance switch had been set or reset, respectively. If the sensor channel has been put into maintenance, the inhibit bit (U.IHB) is set to cease processing on that sensor channel, and the channel is put DOWN. If the channel has been brought out of maintenance, and the INE condition is not set, the inhibit bit is cleared and the processing parameters for that channel are reinitialized to permit the channel to restart.

c. INE bit changed. An "IS" or "IR" message is generated for the station $\log$ to indicate whether the maintenance switch had been set or reset, respectively. If the sensor channel is going into the INE condition, the inhibit bit (U.IHB) is set to terminate processing on that sensor channel, and the channel is put DOWN. The INE condition refers to a state of Invalid data, Equipment sensed; i.e., detection circuits in the sensor and ADACS interface indicate a hardware condition which invalidates the data. When coming out of the INE condition, and the maintenance switch is not set, the inhibit bit is cleared and the processing parameters for that channel are reinitialized to permit the channel to restart.

d. Cardioid configuration changed. The one digit code for the new configuration is logged in a "CC" message on the ASR 28. 
e. Frequency changed. The new frequency setting is logged on the ASR 28 with a "FC" message. The sensor channel is put DOWN, and the processing parameters are reinitialized to permit the channel to come up on the frequency.

When all channels have been checked and all changes logged, control is returned to the system scheduler at E:SKD.

\section{$20.2 \quad$ Called Subroutines}

SUBROUTINE PROGRAM FUNCTION

E:SKD E\$SKD System scheduler reentry point.

E:LOG E $\$ L O G \quad$ Generates coded log messages for the ASR 28.

$20.3 \quad$ Data Formats

20.3.1 Digital Input From IOIS

The first fourteen words of digital input from the IOIS supply the status data on the equipment condition. The format for these inputs, one word per sensor channet, is:

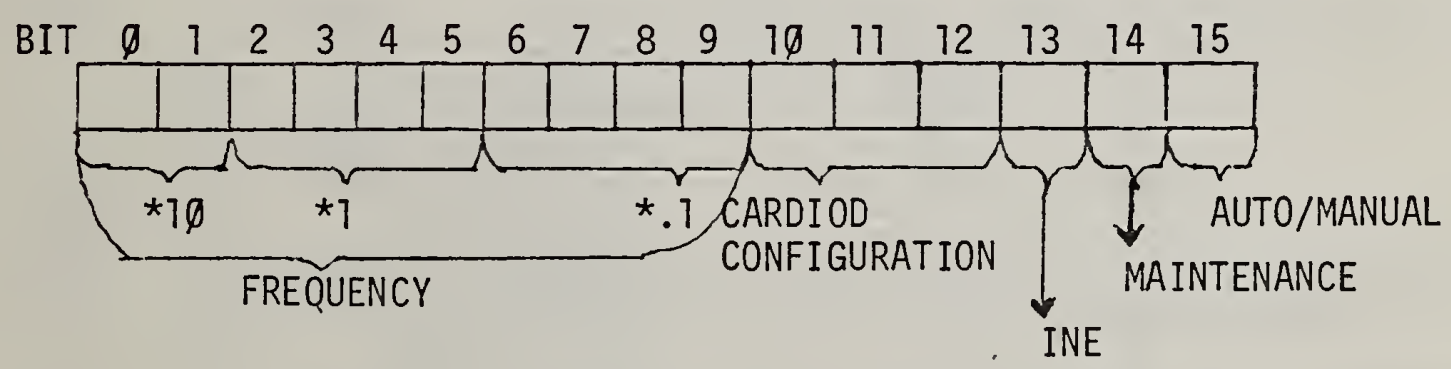

Figure 20.1 Digital IOIS Input Data Format 
The frequency readings are input in 9's complement. The individual digits may be interpreted as follows:

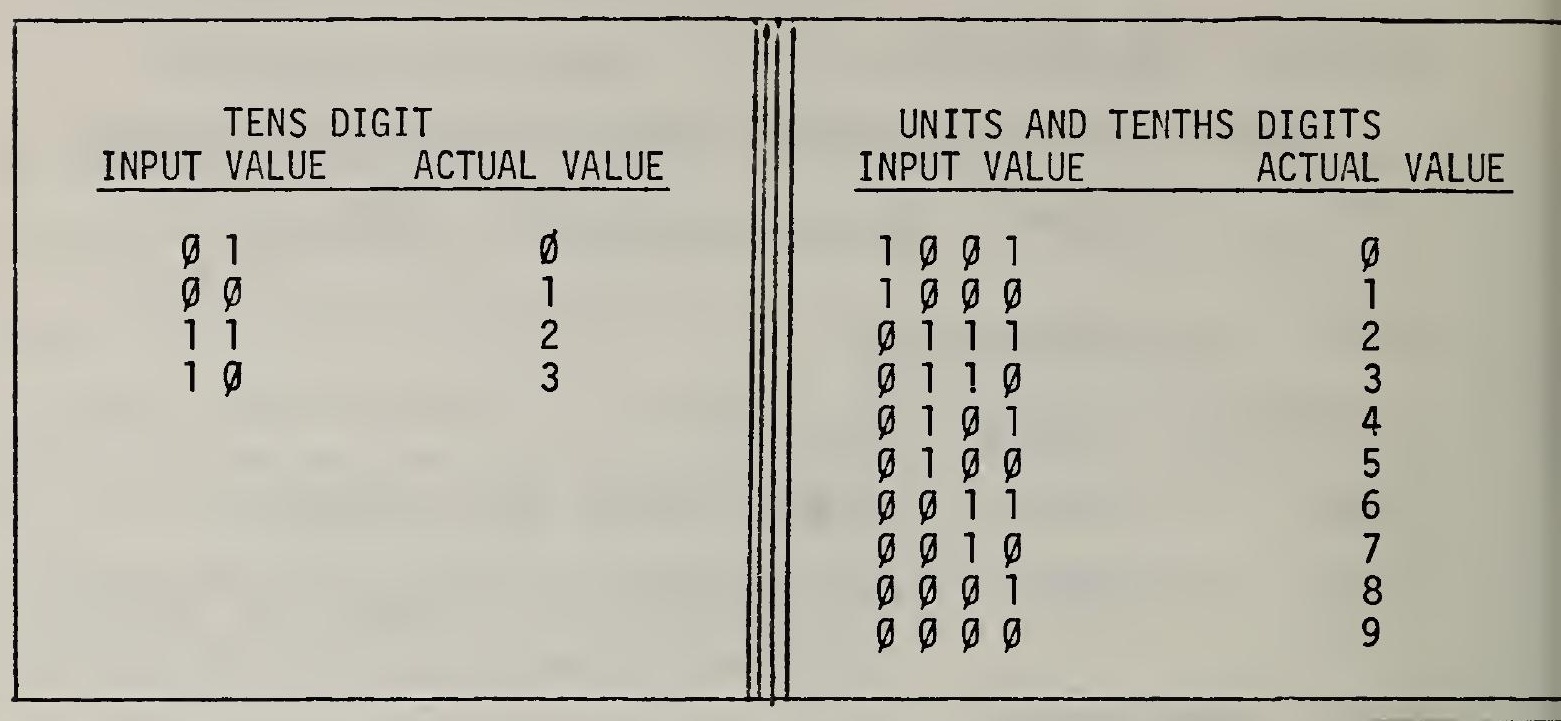

Table $20.1 \quad$ Frequency Value Interpretation

The value which is actually read into the processor is the ones complement of al1 bits except bits 14 and 15. M:ESC first complements bits $\emptyset$ through 13. The cardioid configuration is interpreted as follows:

\begin{tabular}{|cl|}
\hline VALUE & CONFIGURATION \\
\hline$\emptyset$ & LOOP/WHIP NORMAL \\
1 & LOOP NORMAL \\
2 & WHIP NORMAL \\
4 & LOOP/WHIP REVERSE \\
5 & LOOP REVERSE \\
6 & WHIP REVERSE \\
7 & NO CARDIOID USED \\
\hline
\end{tabular}

Table 20.2

Cardioid Configuration Interpretation 
Bits 13, 14, and 15 are interpreted as follows:

$$
\begin{aligned}
\text { Bit } 13 & =\emptyset & & \text { INE condition is RESET } \\
& =1 & & \text { INE condition is SET, processing is inhibited. } \\
\text { Bit } 14 & =\emptyset & & \text { Maintenance condition is RESET. } \\
& =1 & & \text { Maintenance condition is SET, processing is inhibited. } \\
\text { Bit } 15 & =\emptyset & & \text { Sensor channel is operating in AUTO mode. } \\
& =1 & & \text { Sensor channel is operating in MANUAL mode. }
\end{aligned}
$$

\subsection{Input Parameter Descriptions}

PARAMETER PROGRAM DESCRIPTION
U.IHB
U\$DAT
Flag bit set to inhibit processing on a sensor channel if the INE or Maintenance bits are set.
U.CST U\$DAT The channel's processing status, based on the results of the amplitude monitoring section of U:RTA.
U.RTY U\$DAT Counter for consecutive ten-second intervals of "good" data required to bring a channel to the UP state.
J.END U\$DAT Flag to indicate how processing terminated on signal just completed.
U.CLS U\$DAT The class of the waveform being processed, based on the set of criteria met.
U.TRF U\$TGR Flag to indicate the status of the trigger detection program based on the progress of the triggering sequence.
U.OTM U\$DAT Signal onset time, binary format.
U.CCN U\$DAT Accumulated sum of U.CYC to indicate the total effect of cycle shifts induced by NUSTAD.
$\begin{array}{ll}\text { U.TRL U\$TG } & \text { The count of compressed data } \\ \text { trigger waveform analysis. }\end{array}$ 
20.5 Output Parameter Descriptions

PARAMETER SIZE DESCRIPTION

M: INT

Entry point for INE interrupt service routine.

M.TM1 1 Five second delay timer for scheduling M:ESC.

M:ESC Entry point for Equipment Status Change monitoring program.

M.STA 14 Current values for input digital status words, after all changes are logged.

20.6 Internal Data Descriptions

PARAMETER SIZE DESCRIPTION

SWWRD

14 Value of digital input status words from last call to M:ESC. Also called M.STA by external programs.

INWRD $\quad 14$ Buffer of input values read in from first fourteen words of digital input of IOIS during this call to $M: E S C$. 


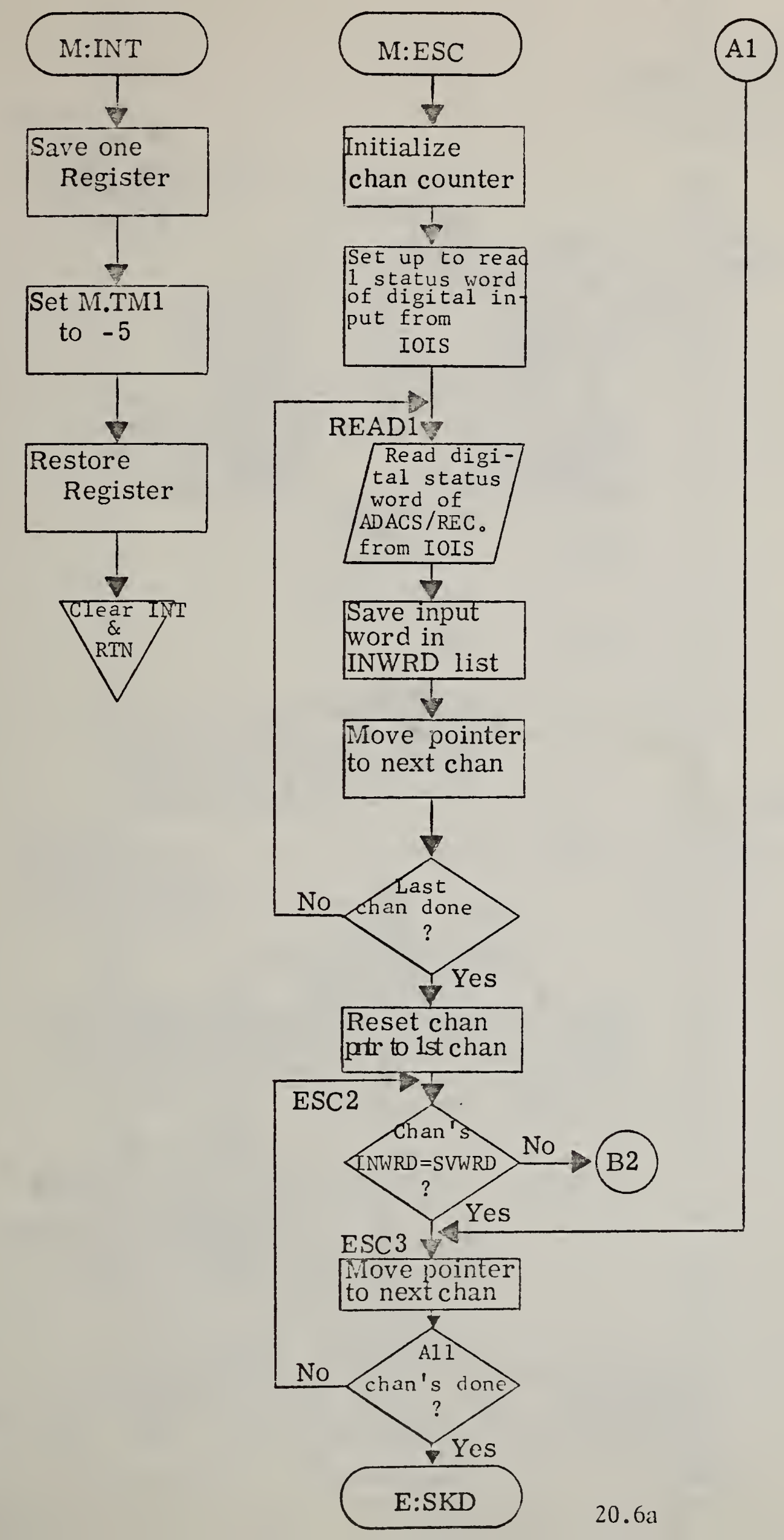




\section{ESC 1}

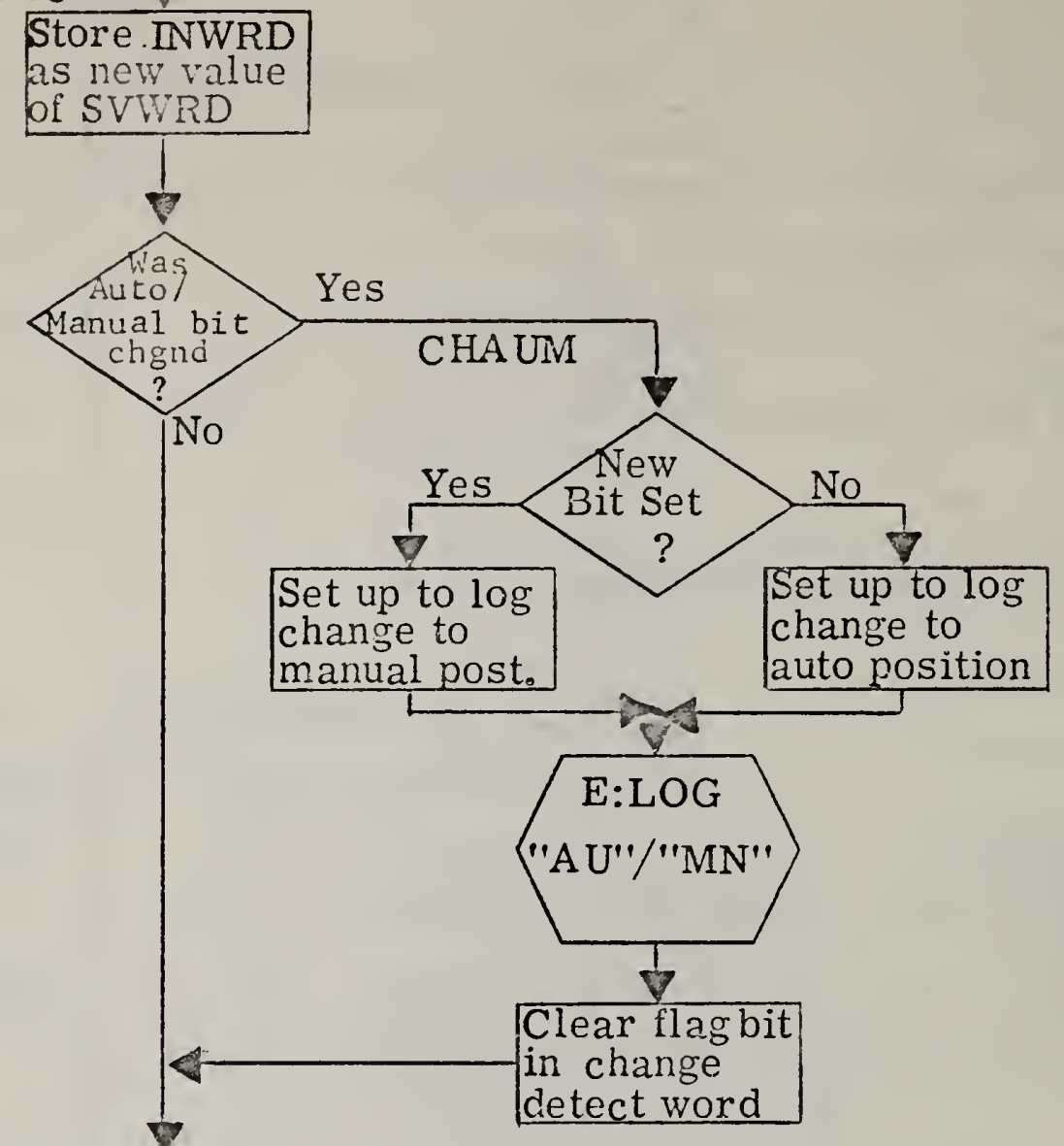

ESC4

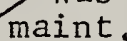

bit chngd

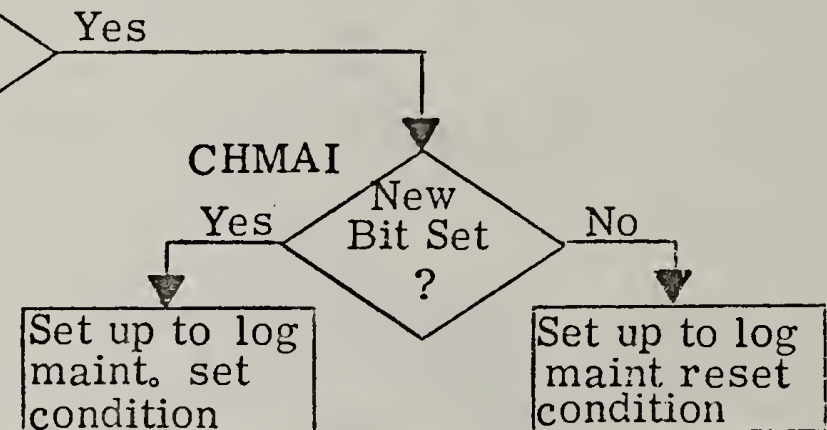

No

condition

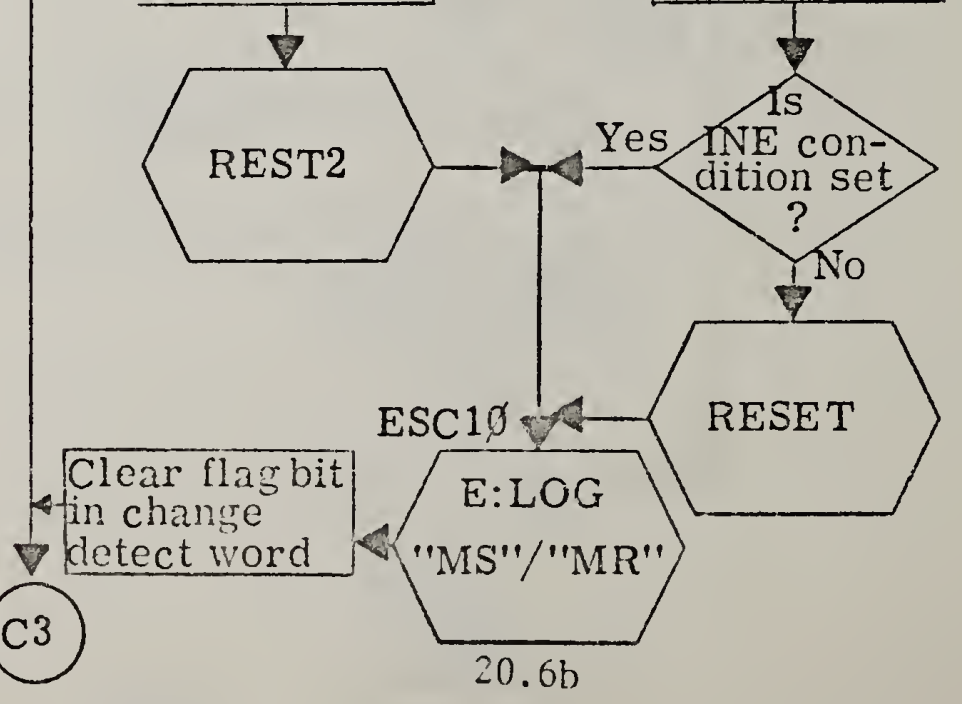

condition 


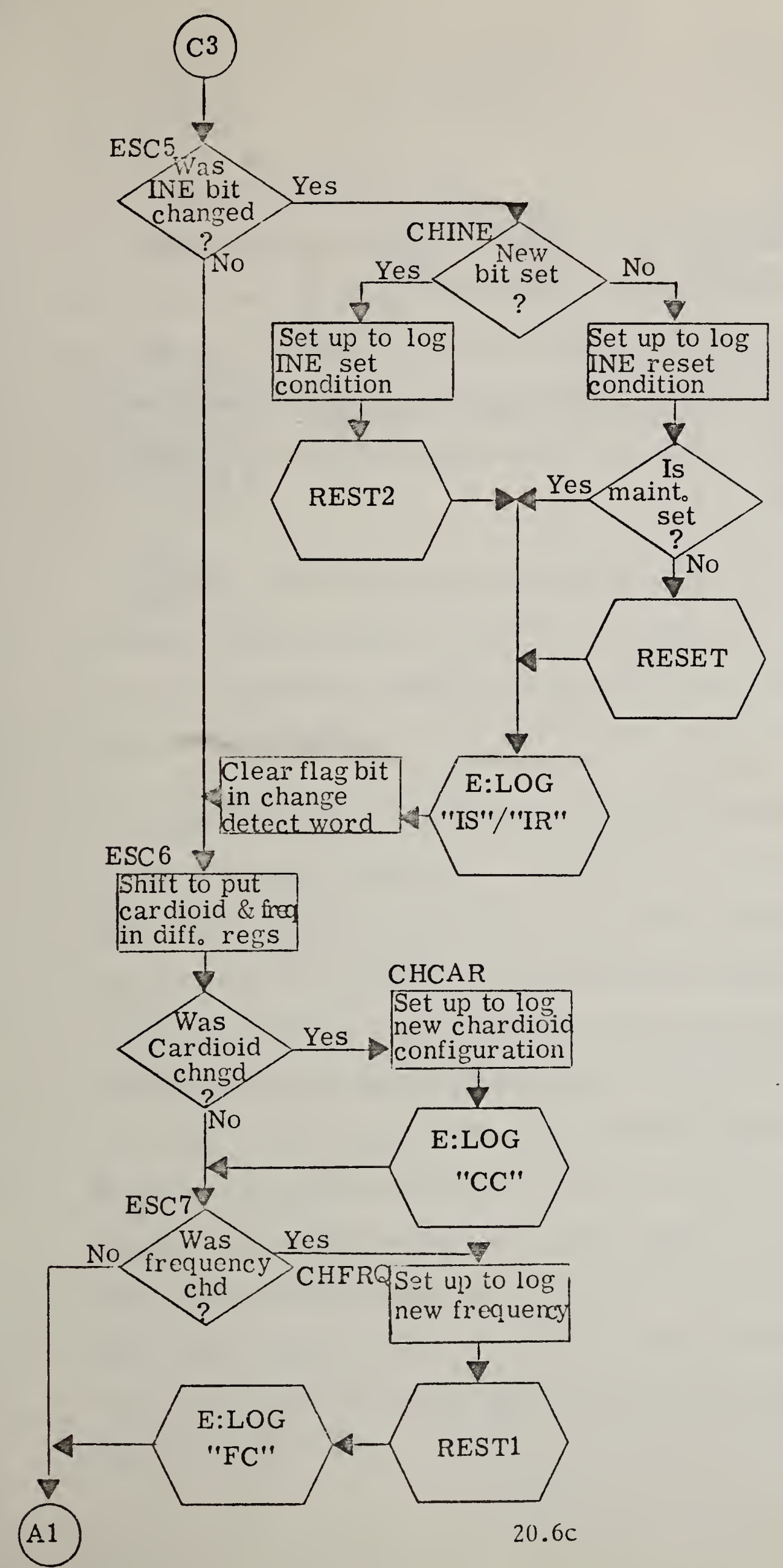

INE bit

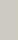



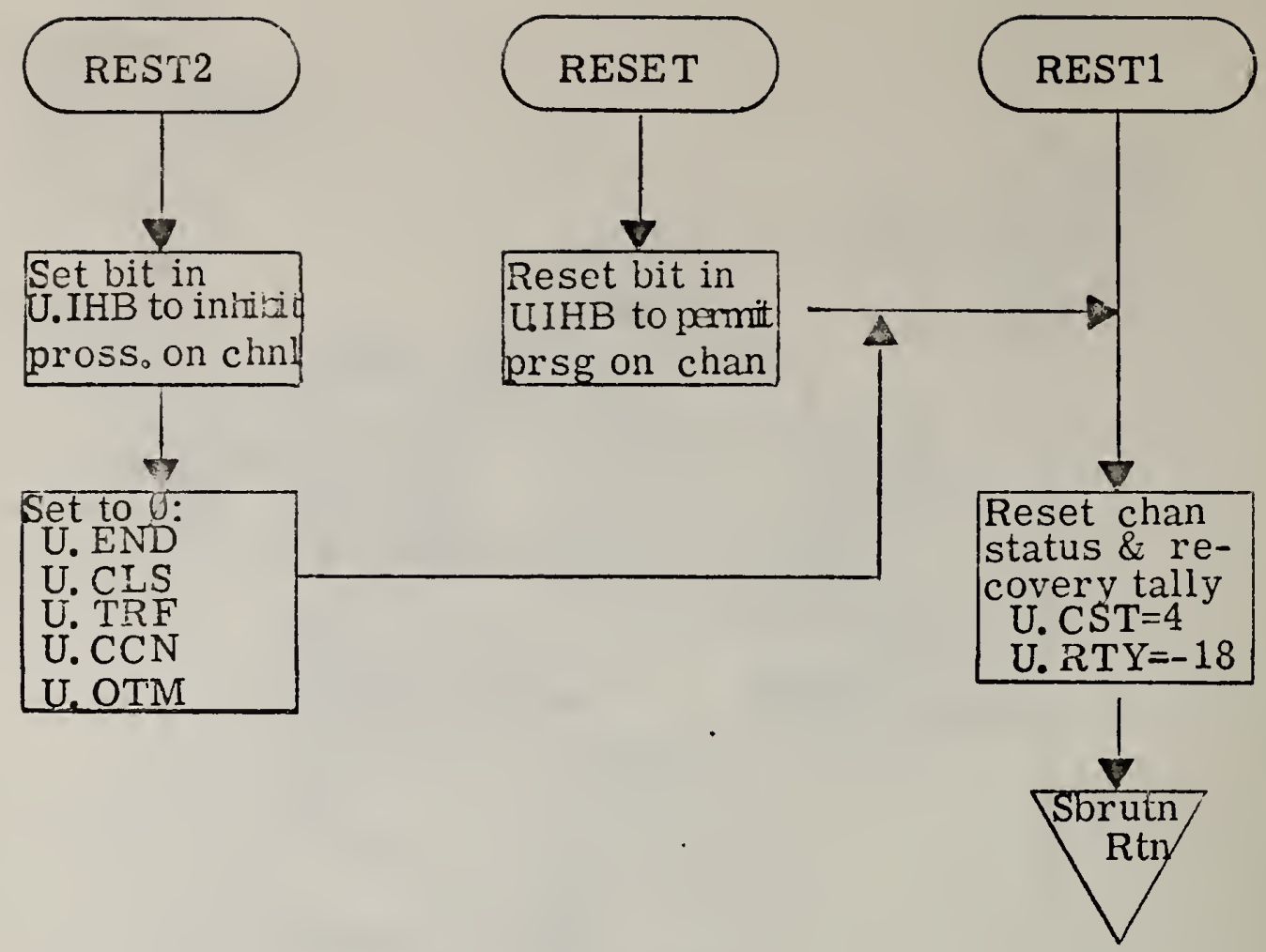
21. M\$LEV

21.1 Functional Description

MSLEV generates a table indicating historical information about each sensor channel which can be used in determining the sensor channel reliability. The table, called the PARAMETER HISTORY, indicates the percentage of time which each channel was in an UP state, and the percentage of up time that each channel was at each tracking rate or gain setting.

$M: L E V$ is entered from the system scheduler when the current time matches an entry in LEVTB of E:MIN (see Section 5.3). E:MIN identifies the need to generate a PARAMETER HISTORY table, and flags M:LEV in the scheduler's task table.

Figure 21.1 shows an example of the output generated by M:LEV on the ASR 28 logging teleprinter. The DOY/TOD group and detachment number are supplied in the healing. The columns correspond to the sensor channels, labeled A through N. The row marked UP gives the percentage of time.since the last-system startup or the last execution of M:LEV, whichever is more recent, that each sensor channel was in the UP state (i.e. U.CST $=1$ ). Under the heading TRACK RATE, each of the possible tracking rate settings corresponds to one row, and the percentage of up time that each sensor channel was at that setting is given in that row. Since there are 25 gain settings, the settings are grouped as shown in Table 21.1. The percentage of channel up time that each sensor channel was in each of the gain groupings is given in the section under the heading of GAIN. 
D24 USJULU RARAMETEK HISTUKY, DET 215

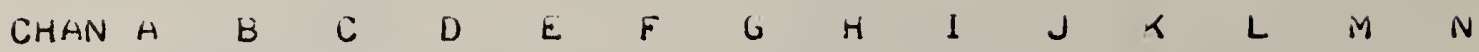
J

TRACS RATE

$\begin{array}{lllllllllllllll}0 & y & 0 & 0 & 12 & 7 & 0 & 1 & 0 & y & 0 & 0 & 0 & y & 0 \\ 1 & 0 & 0 & 0 & 9 & 0 & 0 & 1 & 0 & 0 & 0 & 0 & 0 & 0 & 0\end{array}$ $\begin{array}{lllllrlllllllll}1 & 0 & 0 & 0 & 9 & 0 & 0 & 1 & 0 & 0 & 0 & 0 & 0 & 0 & 0 \\ 2 & 0 & 0 & 0 & 19 & 41 & 0 & 1 & 0 & 0 & 0 & 0 & 0 & 1 & 0\end{array}$

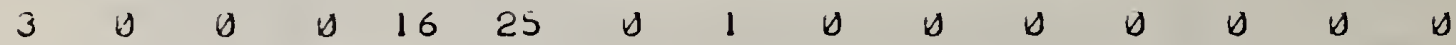

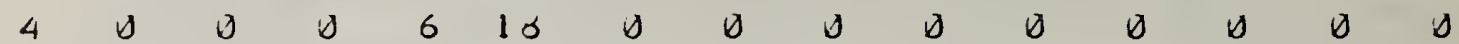

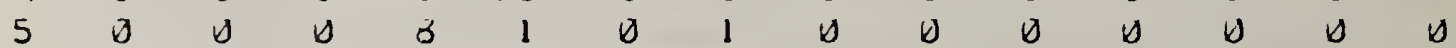
$\begin{array}{lllllllllllllll}6 & 0 & 0 & 0 & 6 & 0 & 0 & 0 & 0 & \theta & 0 & 0 & 0 & 1 & 0\end{array}$

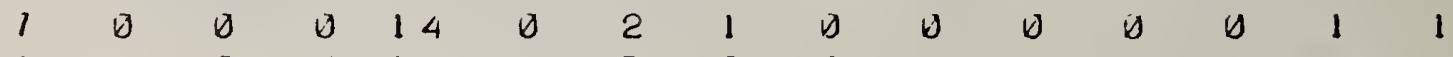
$\begin{array}{lllllllllllllll}1 & 4 & 0 & 0 & 11 & 0 & 5 & 3 & 0 & y & 0 & y & 0 & 0 & 4\end{array}$ $995100100 \quad 0 \quad \forall \quad 93$ y1 $\quad 0 \quad 0 \quad 0 \quad 0 \quad 0 \quad$ yo 95

\section{GHIN}

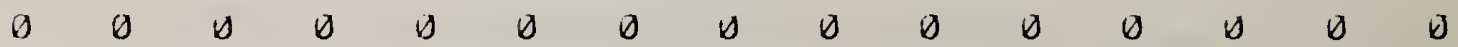

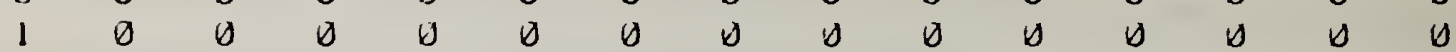

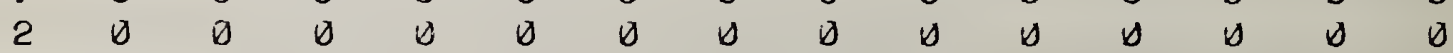

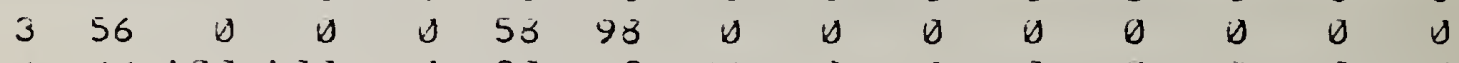

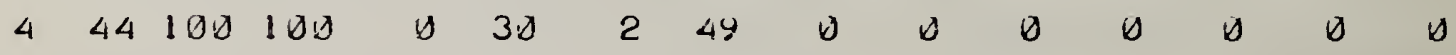

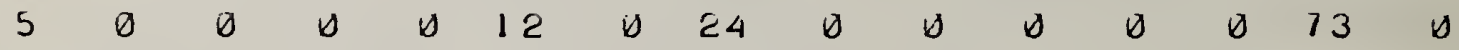

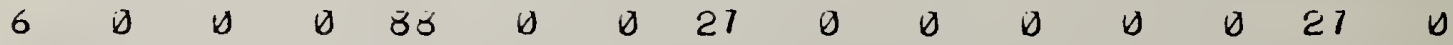

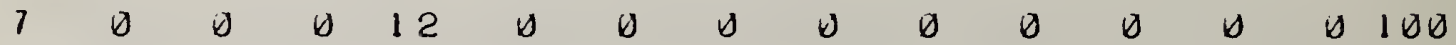

Figure 21.1 Example of PARAMETER HISTORY Table Generated by M:LEV 
When the table is completed, the counters are all reset and control is returned to the system scheduler at E:SKD.

\begin{tabular}{|cl|}
\hline GAIN GROUP & GAIN SETTINGS \\
\hline & $9,1,2$ \\
1 & $3,4,5$ \\
2 & $6,7,8$ \\
3 & $9,19,11$ \\
4 & $12,13,14$ \\
5 & $18,16,17$ \\
6 & $21,22,20,24$ \\
7 & \\
\hline
\end{tabular}

Table 21.1

Organization of Gain Groupings

$21.2 \quad$ Called Subroutines

SUBROUTINE PROGRAM FUNCTION

E:FRT E\$FRT Reformats BCD time from the WTC to ASCII for output.

E:028 E\$T28 Transiates output message to BAUDOT code and transfers message to output buffer before transmission to the ASR 28.

E:BAD E\$BCD Converts binary values to ASCII decima 7 characters for output.

E:SKD E\$SKD Reentry point for system scheduler. 


\subsection{Input Parameter Descriptions}

\section{PARAMETER PROGRAM DESCRIPTION}

E.TM3 E\$CLK Counter for minutes of system up time.

M.UPT E\$CLK Counters for minutes of sensor channel up times.

M.TRT E\$CLK Counters for minutes of channel up time at various tracking rate settings.

M.GNT E\$CLK Counters for minutes of channel up time at various gain group settings.

E.BDF E\$BDC Buffer of ASCII decimal characters generated by $E: B A D$.

E.28F E\$T28 Parameter word used in calling E\$T28

21.4 Output Parameter Descriptions

PARAMETER SIZE DESCRIPTION

M:LEV Entry point for PARAMETER HISTORY table generator.

21.5 Internal Data Descriptions

PARAMETER SIZE DESCRIPTION

$\left.\begin{array}{l}\text { LINE } \emptyset \\ \text { LINET }\end{array}\right\}$

LINE2

LINE3

TERMN

BUFFR

UPFLG

LFLAG

ENDVL

\section{5}

8

5

4

31

14

1

Output buffer for first two lines of output, through column headings.

"TRACK RATE" section heading message.

"GAIN" section heading message.

Five line feed characters used to terminate output.

Output buffer for data lines.

Set to percentage up time, therefore $=\emptyset$ for channels for which TRACK RATE and GAIN section values are all $=\emptyset$.

Bit $\emptyset=\emptyset$ during TRACK RATE section; $=1$ during GAIN section.

Set to highest setting value possible for TRACK RATE and GAIN sections to control number of rows of output in each section. 


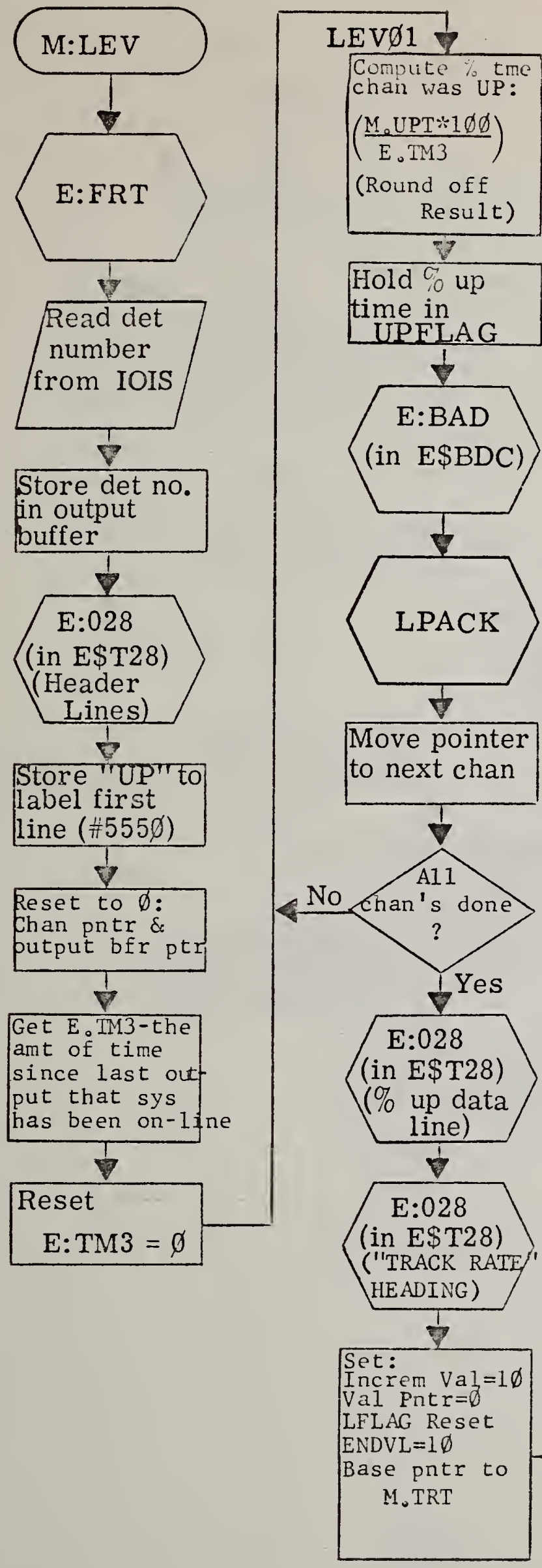

LEV $\emptyset 2$

Reset to Q:

Chan pntr \&

output buffer

pointer

Envrt value

pntr to ASCII

a store for

Qutput

LEVØ3

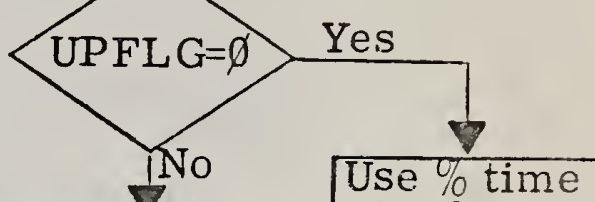

Compute \% tme

chan was at

value:

$\left(\frac{\text { VALUE } * 1 \emptyset \emptyset}{\text { M.UPT }}\right)$

(Rnd off resu] t)

at value as
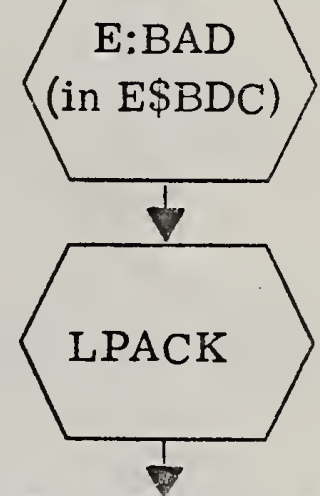

Add increm val

to base pntr

fr next chan

at this level

Increment

chan pointer

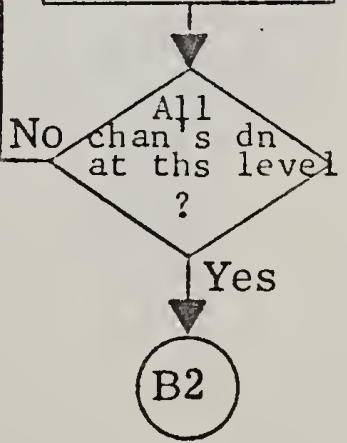




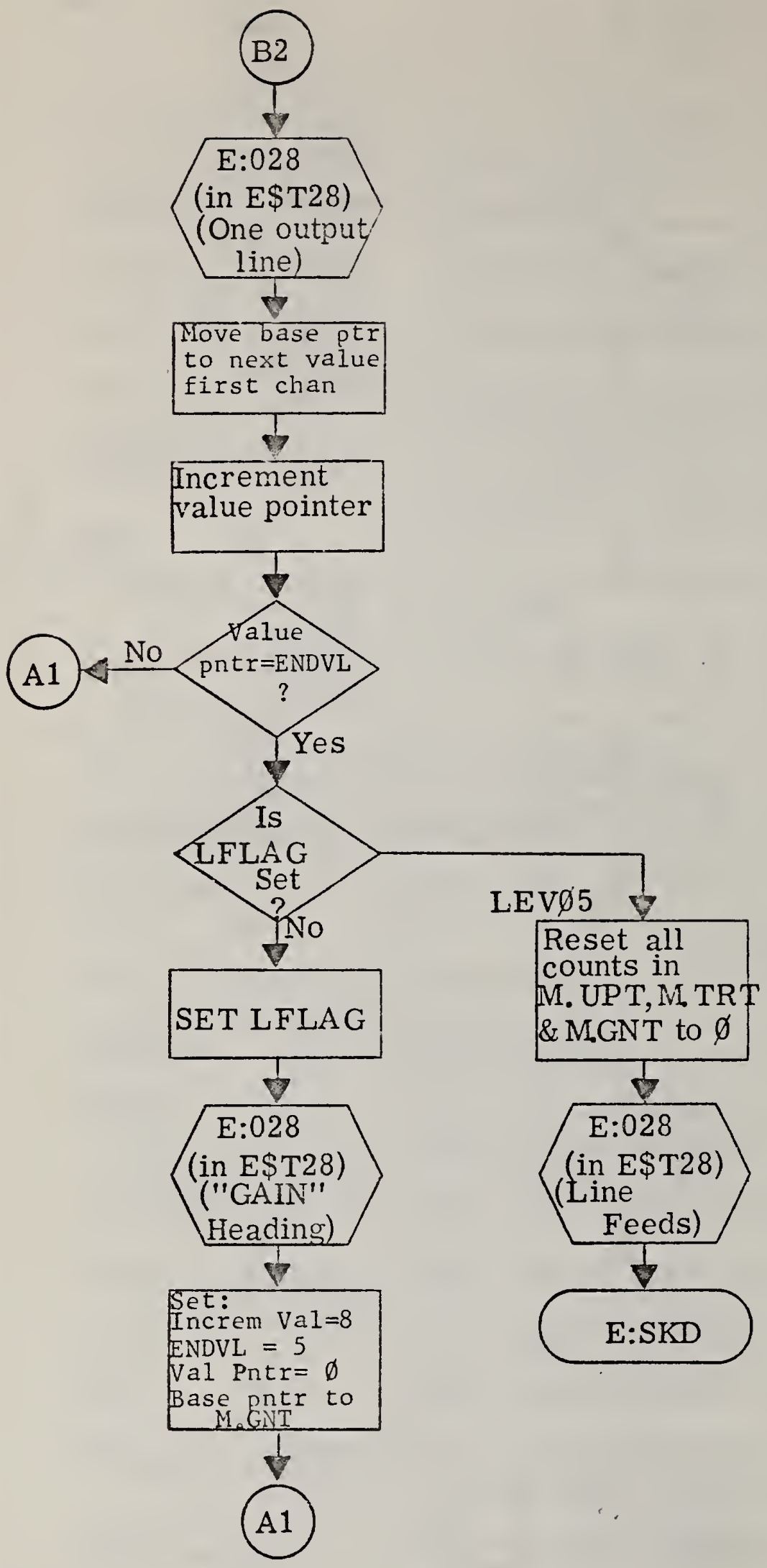




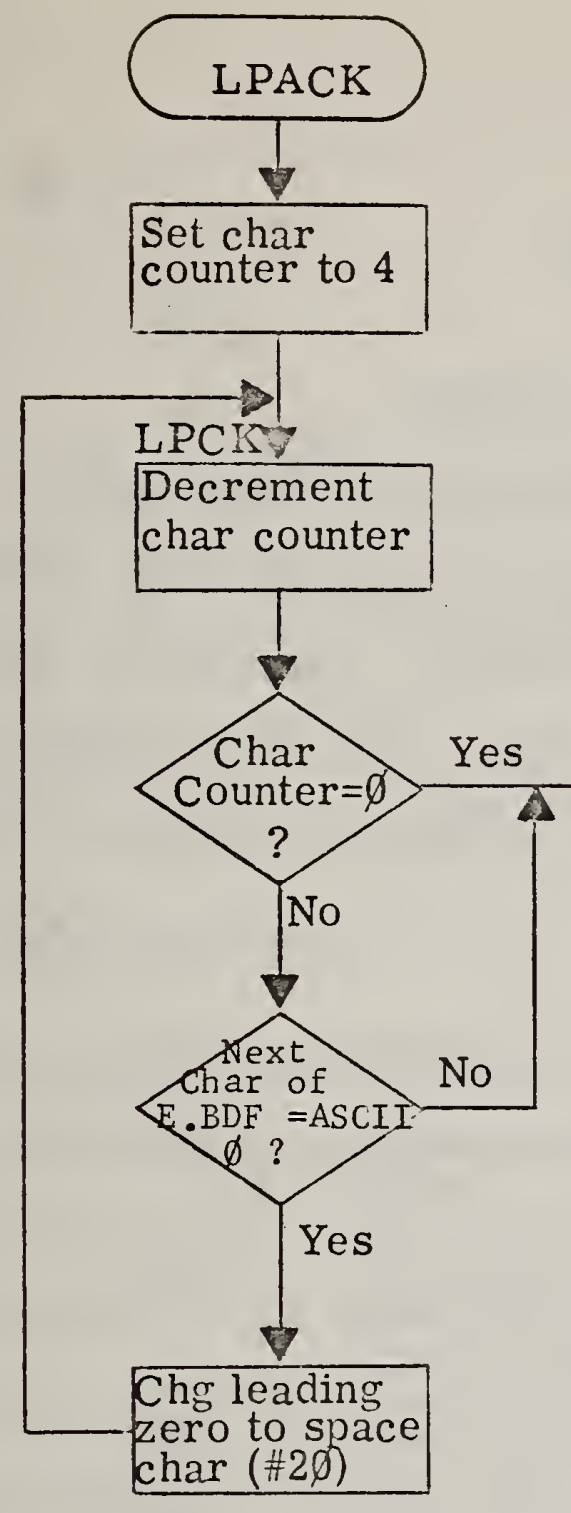

LPCII1

Pack first 2 chars into one word \& move to putput buffer

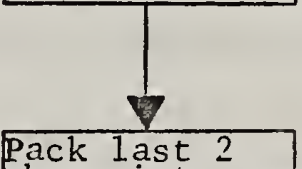

Pack last 2 word \& move to output

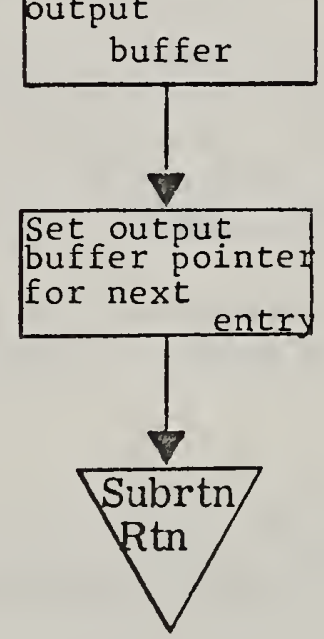


22. M\$STD

22.1 Functional Description

\subsubsection{M:STD (Status Display Routine)}

NUSTAD logs pertinent information about the sensor parameter settings and processing status on the ASR 28 in the form of a table entitied the CURRENT RECEIVER STATUS. This table is generated by M:STD either at predetermined times listed in the STDTB of E:MIN (see Section 5.3 ) or in response to an operator request via E:OMI (the STD: input option).

If the current time, read from the WTC, corresponds to an entry in STDTB of the one minute interrupt service routine (E:MIN) E:MIN sets the flag for M:STD in the scheduler's task table. If the operator enters STD: as the keyword request on the ASR 35 teletypewriter, E:OMI will flag the scheduler task table entry for M:STD. In either way, the scheduler will transfer control to M:STD to generate the CURRENT RECEIVER STATUS table.

After the table is generated, M:STD returns control to the scheduler at E:SKD.

\subsubsection{E:FAM (Amplitude Reformat Subroutine)}

E:FAM is an executive support subroutine which converts amplitude values from internal binary representation to ASCII decimal characters representing the signal amplitude in $\mathrm{dB}$. 
Calling Sequence:

$$
\begin{array}{ll}
\text { BLM, 14 } & \text { E:FAM } \\
\text { DFC } & \text { STORE }
\end{array}
$$

where the value of U.AMP for the channel specified in R6 is converted to ASCII decimal characters and stored in three computer words starting at the location passed in the second statement of the calling sequence (at the location called STORE in the above example). The format of the subroutine output is: $\pm \emptyset \emptyset \emptyset \emptyset$

\subsection{Called Subroutines}

SUBROUTINE PROGRAM FUNCTION

E:FRT E\$FRT Reformats BCD time from the WTC to ASCII for output.

E:028 E\$T28 Translates output message to BAUD0T code and transfers message to output buffer before transmission to the ASR 28.

$E: B A D \quad E \$ B D C$ Binary to ASCII decimal conversion routine.

E:FMF $\quad$ E\$FMF Reformats phase values from internal representation to ASCII decimal for output.

E:SKD E\$SKD Reentry point for the system scheduler.

22.3 Data Formats

Figure 22.1 shows two examples of the CURRENT RECEIVER STATUS tables generated by M:STD. Line 1 includes the DOY/TOD group and the detachment number with the table identification. Each row of the table itself supplies the following information for one sensor channel. 
a. Channel designation, A through $\mathrm{N}$.

b. Frequency, as read from the sensor through the digital input portion of the IOIS.

c. Cardioid configuration, a single digit, interpreted by Table 21.?.

d. INE condition, = $\emptyset$ if INE condition reset; $=1$ if INE condition set.

e. Maintenance condition, $=\varnothing$ if channel not in maintenance; $=1$ if channel is in maintenance.

f. Auto/Manual selection, = A if channel is in Automatic mode; $=M$ if channel is in Manual mode.

If either the INE or the Maintenance conditions are set, the line is terminated after the Auto/Manual indication. Otherwise, the line continues:

g. Gain setting, the current sensor RF gain setting.

h. Tracking Rate setting, the current sensor tracking rate setting.

i. Blanking Level setting, the current sensor blanking level setting.

j. Diurnal slope estimate, the current long term phase trend estimation, given in microseconds/minute. Positive value corresponds to the retard direction, negative to the advance direction. 
U24 W6UU4D CUKRENT RECEIVEK STATUS, UET ¿IS

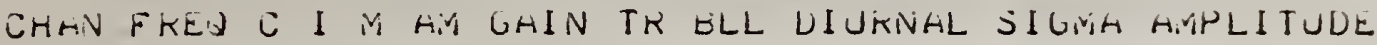

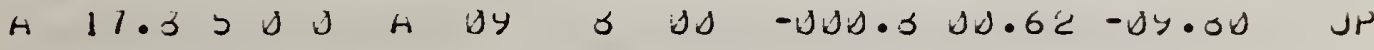

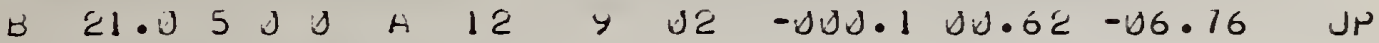

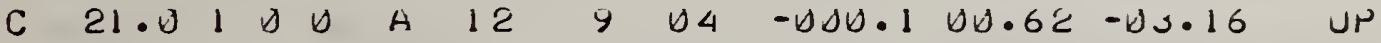

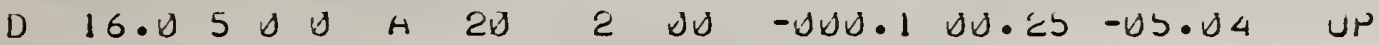

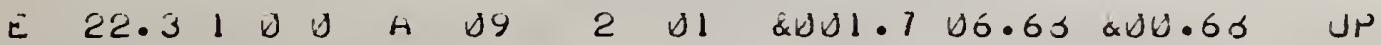

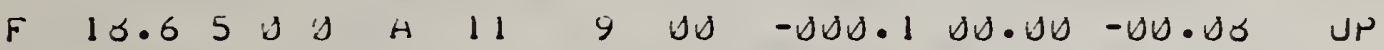

G 24.J 50 U $A$ 2U 3 UD -0.0 .7 U. $31-01.24 \quad U P$

H ZERO INRUT...

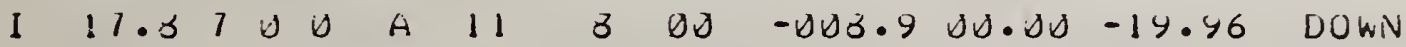

J ZEKO INPJT...

K ZEKO INPJT...

L ZERO INPJT...

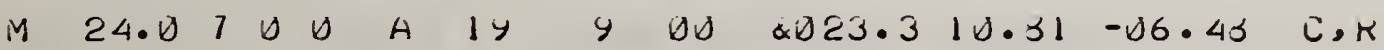

$N \quad 17.3 \quad 7 \quad 0 \quad 0 \quad A \quad 24 \quad 6$ DU

\24 D9O153 CJRKENT RECEIVEK STATUS, DET ¿ 15

CHAN FKEJ C I M AM GAIN TR BLL DIJKNAL SIGMA AMPLITUDE

\begin{tabular}{|c|c|c|c|c|c|c|c|c|c|c|}
\hline 17.8 & 50 & ע & $A$ & 12 & 9 & ப் & -பலb.1 & $\Delta U .18$ & $-63 \cdot 04$ & مل \\
\hline $21 \cdot 1$ & 50 & 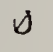 & $A$ & 16 & 1 & 16 & \&むபம. 6 & 03.00 & $-.55 \cdot 36$ & نे \\
\hline $21 \cdot 1$ & $1 \sqcup$ & $\Delta$ & $A$ & 14 & 1 & 17 & \&ubu. 7 & d3. & $-\Delta 6 \cdot 46$ & Pr \\
\hline 16.0 & $5 y$ & $D$ & $A$ & 22 & 2 & $\Delta \circlearrowright$ & $\& \circlearrowright \sqcup \sqcup \cdot \Downarrow$ & $\Delta U . \sigma 1$ & $-81 \cdot 76$ & PU \\
\hline $22 \cdot 3$ & $1 \mathrm{v}$ & $v$ & $H$ & 11 & 1 & 01 & \&UV1.5 & $\Delta . \cdot 25$ & $-000.6 \sigma$ & קט \\
\hline 16.6 & 50 & $\Delta$ & $A$ & 11 & $\dot{y}$ & עb & 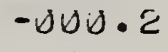 & 06.06 & $4 b D \cdot 16$ & RU \\
\hline 24.0 & 50 & $v$ & $A$ & 16 & $y$ & $\sqcup \sqcup$ & $-\sqcup \sqcup \sqcup \cdot 1$ & טU. 25 & . 52 & \\
\hline
\end{tabular}

H ZKKO INPJT...

$\begin{array}{lllllllll} & 1 & 1 & 8 & 7 & 1 & 1 & M\end{array}$

J ZEKO INPJT...

K ZEKO INPJT...

L ZEKKO INPJT...

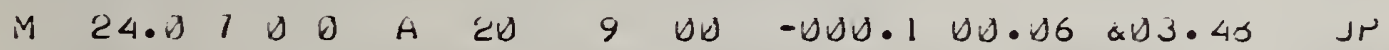

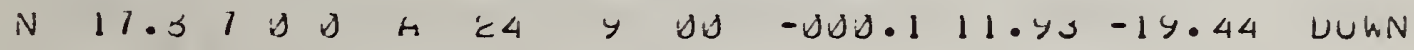

Figure 22.1 Two Examples of CURRENT RECEIVER STATUS Tables Generated by M:STD 
k. Standard Deviation estimate, the current standard deviation value for the incoming phase signal, given in microseconds.

1. Amplitude value, the current signal amplitude in $d B$.

m. Channel processing status, the channel processing status based on the amplitude evaluation; UP if the channel is up; C,NR if the channel is coasting, no report; $C, R$, if the channel is coasting, and the reason has been reported in the station log; and DOWN if the channel is down.

Additional7y, if the channel's digitial IOIS data word is zero, the INE and MAINTENANCE conditions are set by default. This state is entered as a

$$
\text { ZERO INPUT... }
$$

entry in the table.

\subsection{Input Parameter Description}

PARAMETER PROGRAM DESCRIPTION
E.28F
$E \$ T 28$
Parameter word used in calling E:028
E.BSG
$E \$ B D C$
The ASCII representation for the sign of the output of E:BAD.
E.BDF
$E \$ B D C$
The buffer of ASCII decimal characters resulting from $E: B A D$.
U.GAN U\$DAT The current sensor channel RF gain settings.
U.TRK U\$DAT The current sensor channel tracking rate settings.
U.BLL USDAT The current sensor channel blanking level settings.
U.SIG USDAT The computed standard deviation of the incoming phase signals. 
22.4 Input Parameter Description (continued)

PARAMETER PROGRAM DESCRIPTION

U.FREQ E\$LOG The current sensor channel frequency settings.

U.AMP U\$DAT The current signal amplitude estimates.

M.STA M\$LEV The current digital IOIS status from the sensors and ADACS interfaces.

U.IHB U\$DAT Flag bit used to inhibit processing on channels with either INE or Maintenance conditions set.

U.CST U\$DAT Channel processing status based on amplitude evaluation by amplitude monitoring section of U:RTA

22.5 Output Parameter Descriptions

PARAMETER SIZE DESCRIPTION

M:STD Entry point to CURRENT RECEIVER STATUS table generation program.

E:FAM

Entry point to executive support subroutine to reformat amplitude values to ASCII characters of $\mathrm{dB}$.

22.6 Internal Data Descriptions

PARAMETER SIZE DESCRIPTION

LINE $\emptyset$ LINE $54 \quad$ First two lines of table, through column headings.

LINE2

L2A1

31

LINE3

11

TERMN

3

TEMP

5

STAT

8 Table to convert U.CST from numeric to characters for output. 

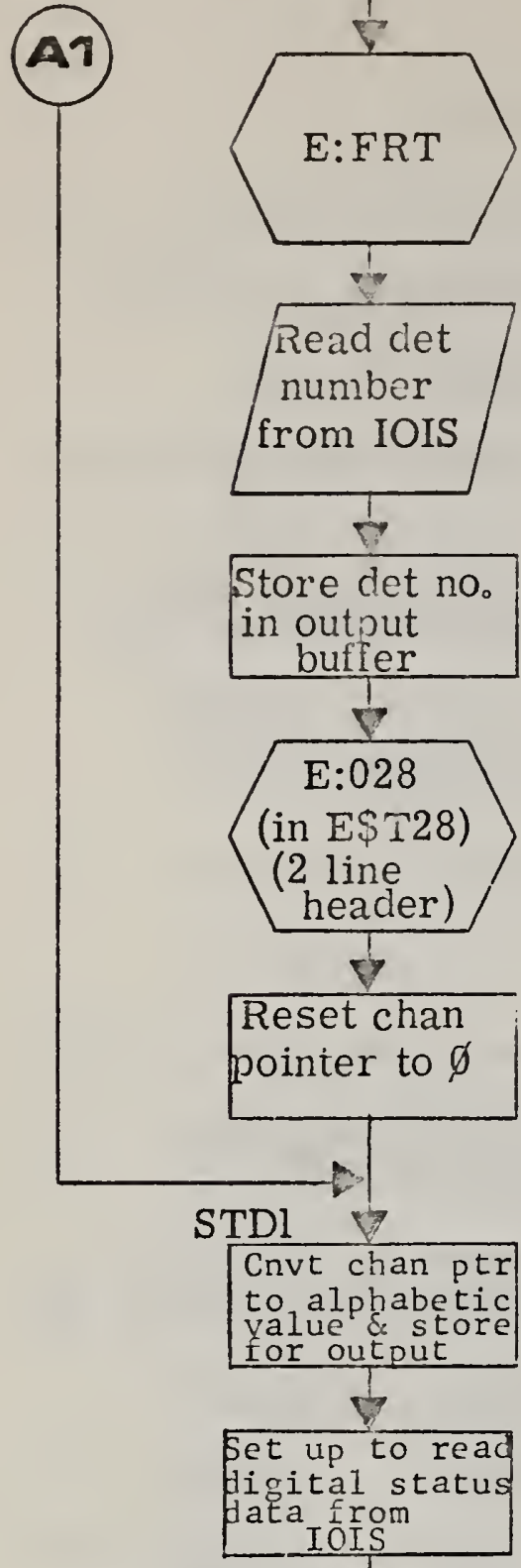

READ1

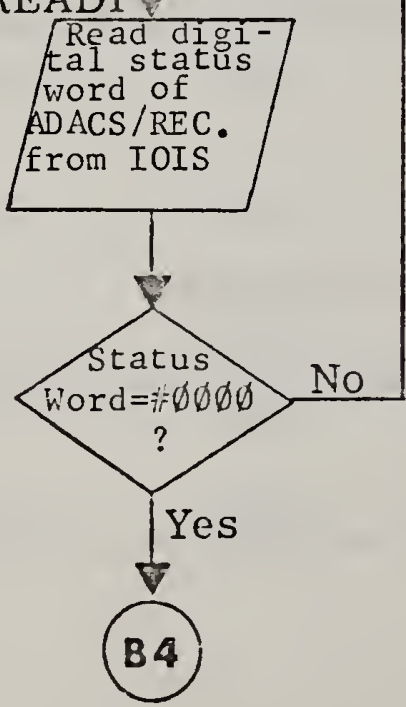

Hold status word in M.SIA

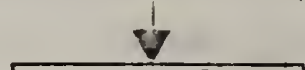

Extract freq $\mathrm{fm}$ status wrd \& str in UoFRQ and in output buffer

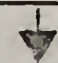

Extract 3 bIt cardioid value \& str in output buffer

Copy INE bit into holding register

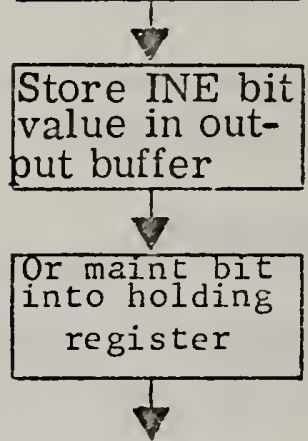

Str maint bit value in output buffer
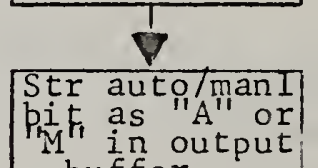

buffer

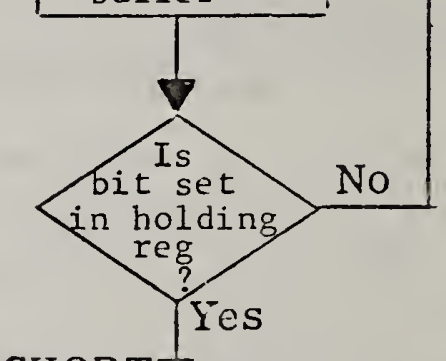

SHORT

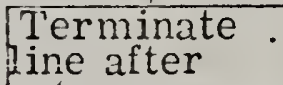

ine after

A/IVI value

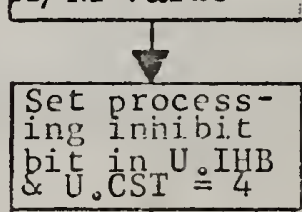

Cnvt U.GAN $\mathrm{fm}$

binary toASCI

decimal \& str

for output

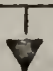

CnVt U.TRK fm

Binary to ASCII

decimal \& str

for output

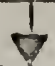

Cnvt U BLL fm Binary to ASCII decimal \& str for outout

Get U.DIU \& scale diurnal slope to 1 usec/minute

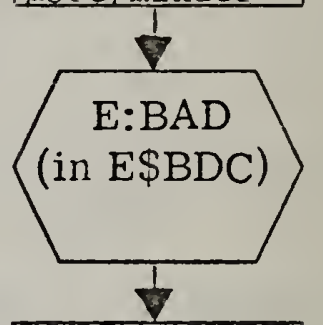

Pack \& store signed diumal slp fr output

Get U. SIG (Standard Deviation)

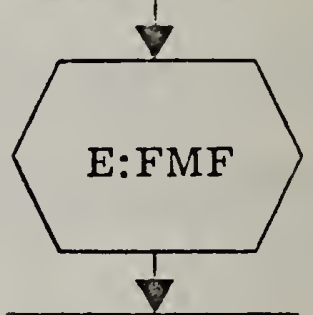

Store away sigma for

output

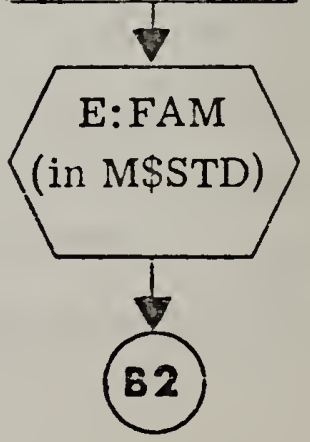




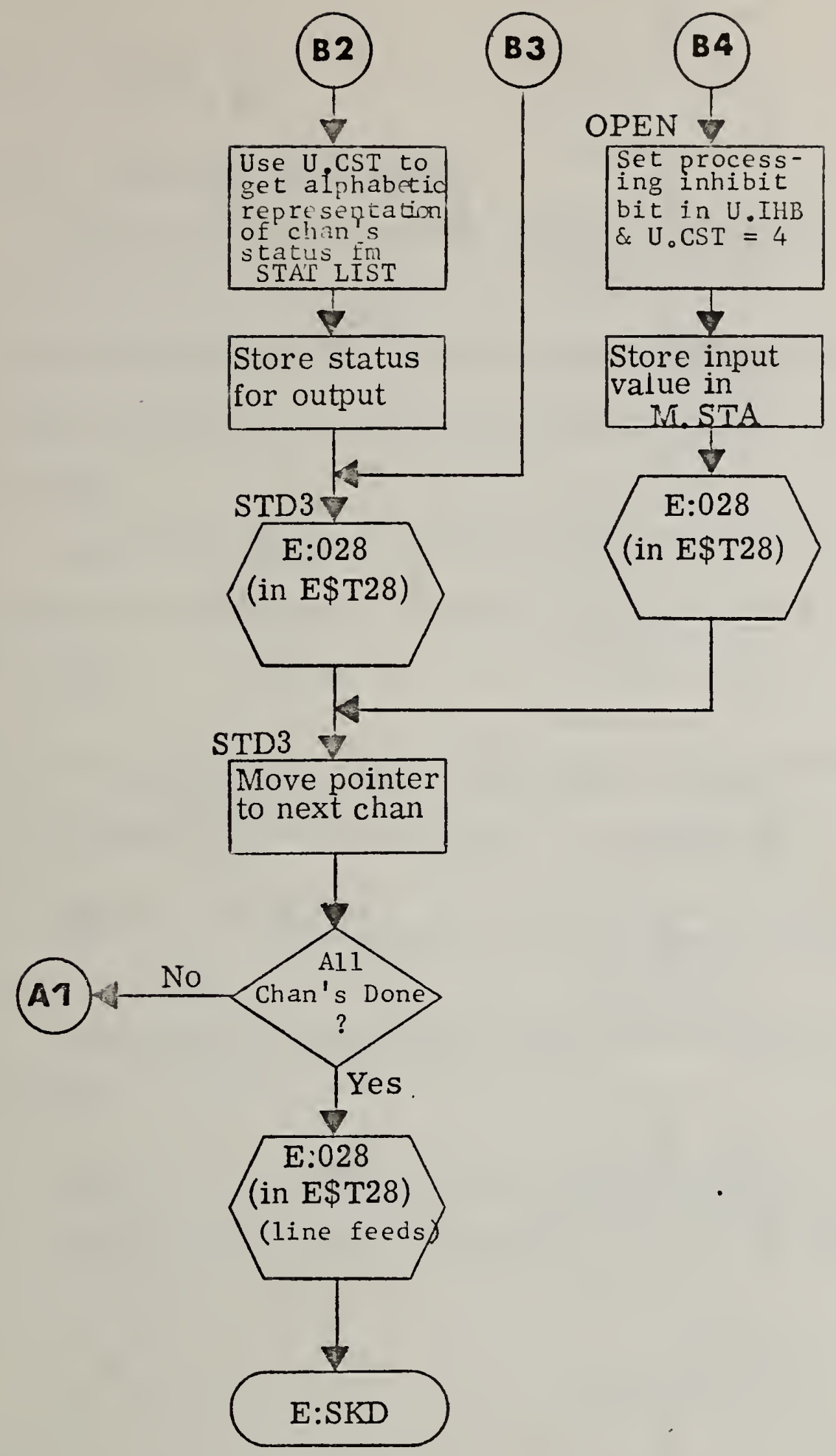




\section{E:FAM}

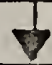

SAVE REGISTER

$1-7$

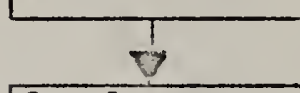

Get Loc。to

store output

from calling

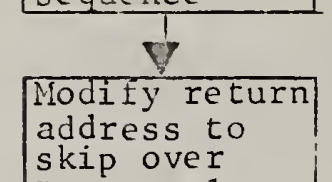

skip over

Parmo value

Save return

Addr .register

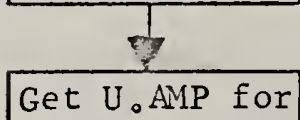

this chan.

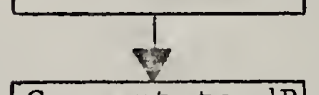

Convert to $\mathrm{dB}$

$\left(\frac{U}{0}\right.$ AMPx $\left.1 \emptyset \emptyset \emptyset\right)$

$\left(\frac{0.4 M \times 1000}{2048}\right)-$
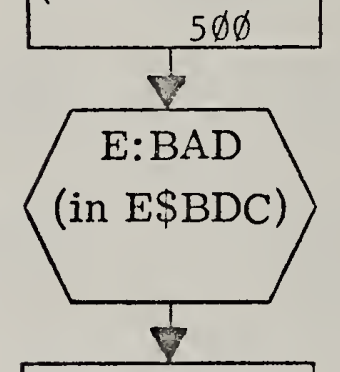

Pack signed

result \& str

for output

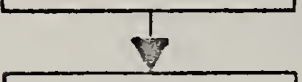

RESTORE

REGISTERS

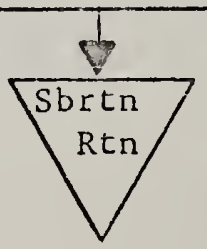


23. E\$LOG

\subsection{Functional Description}

Routine messages concerning changes to parameter settings, etc. are entered into the station $\log$ on the ASR 28 in a coded form. Systems and applications programs generate these messages by calling $E: L O G$, the executive support subroutine to generate routine $\mathrm{log}$ mess ages.

The calling sequence depends on whether or not a new value is appended to the message. The basic calling sequence is:
BLM, 4
$E: L O G$

If further information is required, as in a parameter change message, it is passed in a second word, as:

$\begin{array}{ll}B L M, 4 & E: L O G \\ D F C & \text { (New value or other additional information) }\end{array}$

The message text and sensor channel are identified in R5 as follows:

Bits $\emptyset-7 \ldots$ Channel designation, $\emptyset$ for chan $A, I$ for chan $B$, etc. Bits 8 - 15.....Message designation number, see Table 23.1 


\begin{tabular}{|c|c|c|c|}
\hline $9 \emptyset$ & "U" & Channel UP & $\begin{array}{l}\text { Frequency, Track Rate, Gain, } \\
\text { Blank Lv }\end{array}$ \\
\hline 01 & "FC" & Frequency change & New frequency setting \\
\hline$\emptyset 2$ & "CC" & Cardioid change & New cardioid pattern code \\
\hline 93 & "TC" & Tracking rate change & New tracking rate \\
\hline 94 & "GC" & Gain change & New gain setting \\
\hline 65 & "BC" & Blanking Level change & New blanking level setting \\
\hline 96 & "RS" & Redundancy check successfu & 11 None \\
\hline 97 & "SD" & System Dead in scheduler & None \\
\hline$\emptyset 8$ & "MN" & Auto/Manual set to Manual & None \\
\hline 99 & "AU" & Auto/Manual set to Auto & None \\
\hline 10 & "MS" & Maintenance switch set & None \\
\hline 11 & "MR" & Maintenance switch reset & None \\
\hline 12 & "IS" & INE condition set & None \\
\hline 13 & "IR" & INE condition reset & None \\
\hline 14 & "CF" & Channel coasting, failure & None \\
\hline 15 & "CN" & Channel coasting, noise & None \\
\hline 16 & "CS" & Channel coasting, saturati & None \\
\hline 17 & "D" & Channel Down & None \\
\hline 18 & "AF" & Analog subsystem failure & None \\
\hline 19 & "PF" & Power failure & None \\
\hline $2 \emptyset$ & "PR" & Power restored & None \\
\hline 21 & "MP" & Memory parity error & Memory location of error \\
\hline
\end{tabular}

Table 23.1 Message Number Designation and Interpretation

After the message is generated and transferred to the ASR 28 output buffer via $E: 028$, control is returned to the calling program.

\subsection{Called Subroutines}

SUBROUTINE PROGRAM FUNCTION
$E: 028$
E\$T28
Translates output message to BAUDOT code and transfers message to output buffer before trans- mission to the ASR 28.
$E: F R T \quad E \$ F R T$
Reformats BCD time from the WTC to ASCII for output.
$E: B A D \quad E \$ B D C$
Binary to ASCII decimal conversion subroutine 


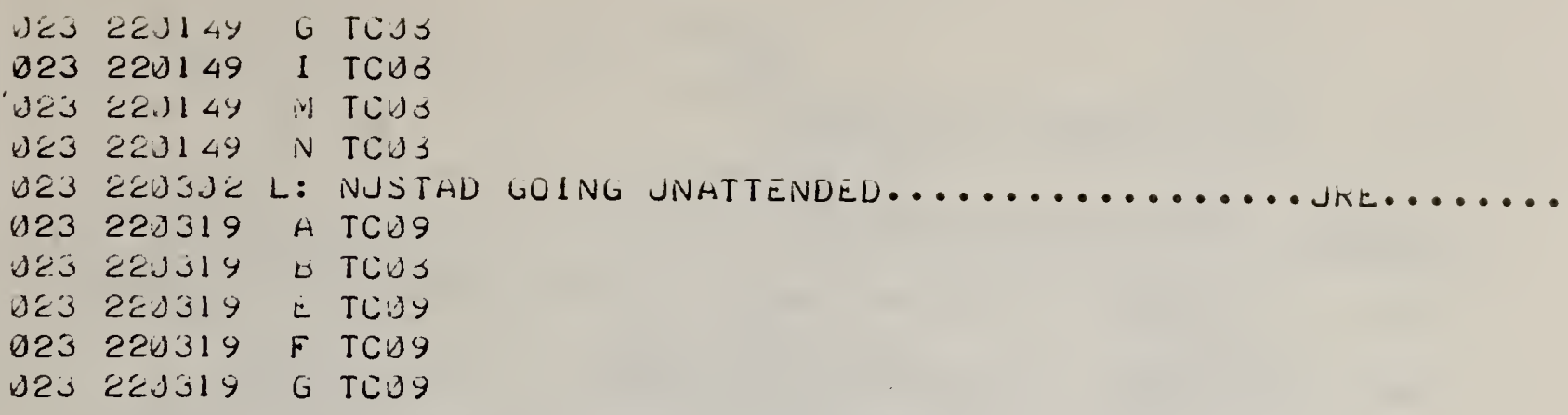

Figure 23.1 Example of Routine Log Messages

\subsection{Data Formats}

The routine log message, as illustrated in Figure 23.1, assumes the following format:

DDD TTTTTT C MMAA

where: $\quad \underline{D}$ indicates a digit of DOY

T indicates a digit of TOD

$\underline{C}$ indicates an alphabetic designation for sensor channel

M indicates one of two characters giving the message text, see table 23.1 for interpretation of the legal messages.

A indicates a character of additional information which may be associated with the message text.

NOTE: Line 5 of Figure 23.1 illustrates an example of the output generated by the operator LOG: option (see Section 8.1). 
23.4 Input Parameter Descriptions

PARAMETER PROGRAM DESCRIPTION

U.TRK U\$DAT Current sensor channei tracking rate settings.

U.GAN U\$DAT Current sensor channel RF gain settings.

U.BLL U\$DAT Current sensor channel blanking level settings.

E.BDF $\quad E \$ B D C \quad$ Buffer of ASCII decimal characters from call to $E: B A D$

E.28F E\$T28 Parameter word used in calling E:028

23.5 Output Parameter Descriptions

PARAMETER SIZE DESCRIPTION

E:LOG Entry point to routine log message generator.

U.FRQ $28 \quad$ ASCII characters for current sensor frequency settings.

23.6 Internal Data Descriptions

PARAMETER SIZE DESCRIPTION

MSGTB 22 Table of the character message texts for the routine $\log$ messages.

OBUFF $\quad 17 \quad$ Message output buffer.

OBUF1

SAVRS 7 Register storage area. 


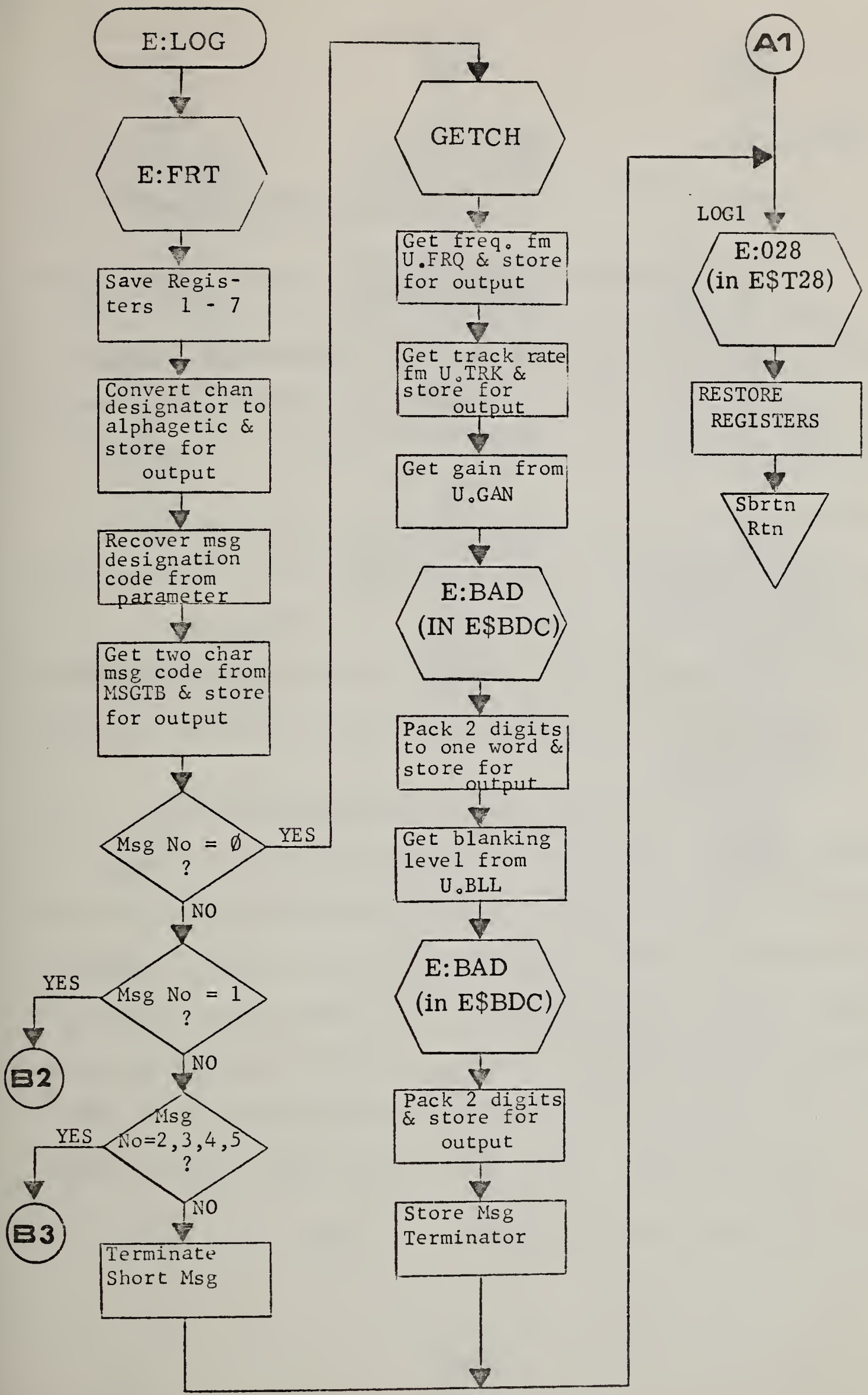



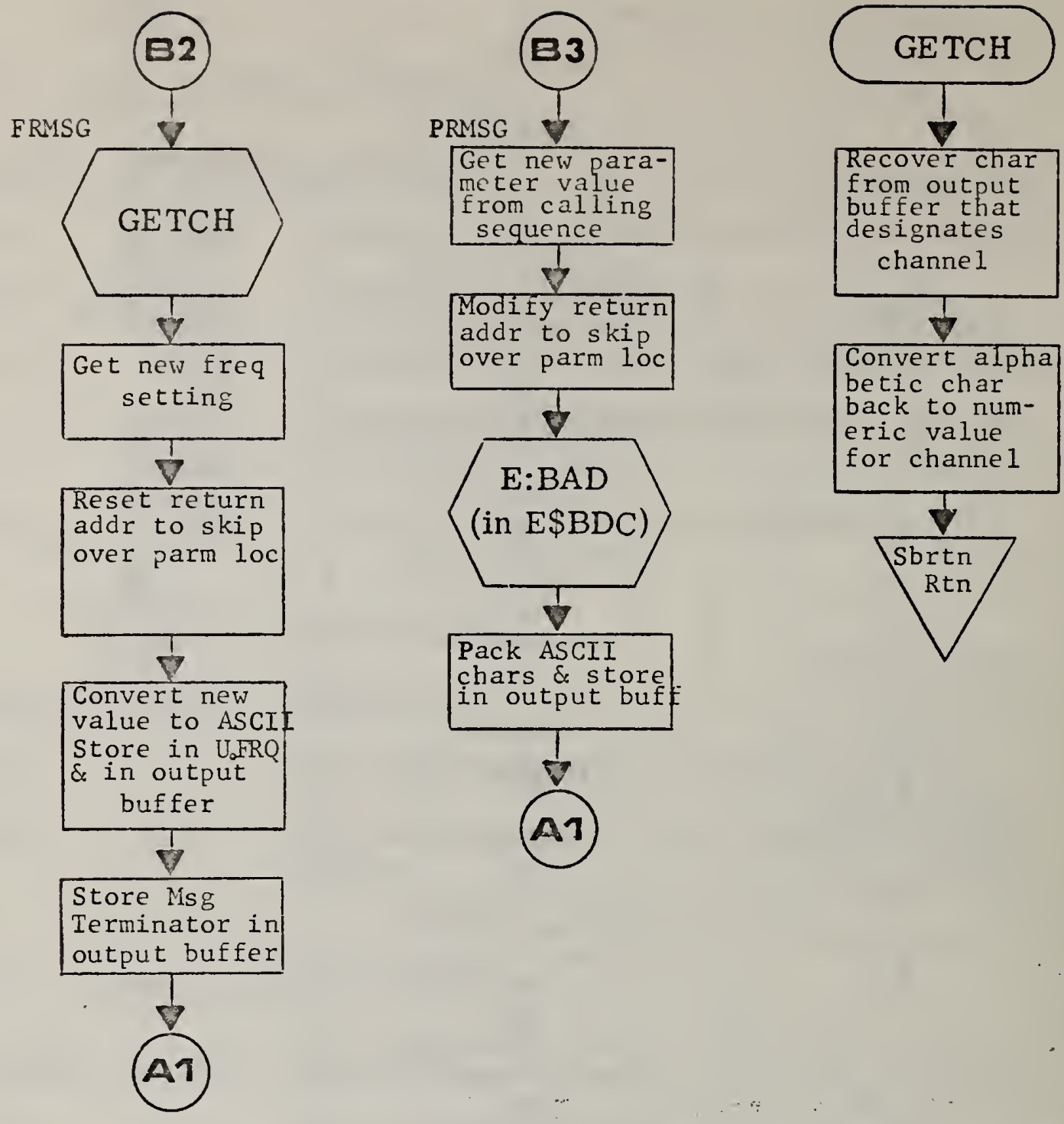

Convert alpha betic char back to numeric value for channe 1

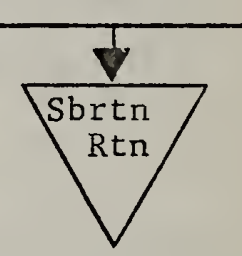

chars \& store

Terminator in 
24. $E \$ B D C$

24.1 Functional Description

24.1.1 E:BDC (Binary to Decimal Conversion Subroutine)

$E: B D C$ is an executive support subroutine designed to convert binary values to $B C D$ (decimal) equivalents. The calling sequence is:

$B L M, 14$. E:BDC Binary value to be converted in $\mathrm{R} 12$

The sign of the value is stored in E.BSG as either ${ }^{202 B_{16}}$ (the ASCII characters for " + ") for positive values or $202 \mathrm{D}_{16}$ (the ASCII characters for " - ") for negative. The least significant five digits of the resulting $B C D$ value are stored in $E . B D F$, the least significant digit first. After the conversion, control is returned to the calling program.

\subsubsection{E:BAD (Binary to ASCII Decimal Conversion Subroutine)}

Normally a binary to decimal conversion is made to generate output data in a form usable by the operator. E:BAD performs the same conversion as $E: B D C$, but also converts the BCD digits to ASCII characters during the process to have them in the form compatible with standard output devices. The sign is stored in E.BSG and the least significant five digits of the resulting value are stored in $E . B D F$ as with $E: B D C$. These values are ASCII rather than BCD characters. Calling sequence is:
BLM, 14
$E: B A D$
Binary value to be converted in $\mathrm{R} 12$ 


\subsection{Computational Methods}

The binary to $B C D$ conversion is made by successive divisions by ten. The conversion from BCD to ASCII is made by adding $3 \emptyset_{16}$ to each digit.

\subsection{Output Parameter Descriptions}

\section{PARAMETER SIZE DESCRIPTION}
$E: B D C$
Entry point for binary to decimal conversion routine.
$E: B A D$
Entry point for binary to ASCII decimal conversion routine.
$E: B D F$
5 Output character buffer for BDC or ASCII digits, arranged with least significant digit first, etc.
$E: B S G$

$$
\begin{aligned}
& 1=2{ }^{2} B_{16} \quad \text { (ASCII " }+" \text { ) if value is positive; } \\
& -292 D_{16} \text { (ASCII " }-" \text { ) if value is negative. }
\end{aligned}
$$

\subsection{Internal Data Descriptions}

PARAMETER SIZE DESCRIPTION

CONVR

1 Bit $\emptyset=\emptyset$ for BCD output, $=1$ for ASCII decimal output.

SAVRS $\quad 7$ Register storage area. 


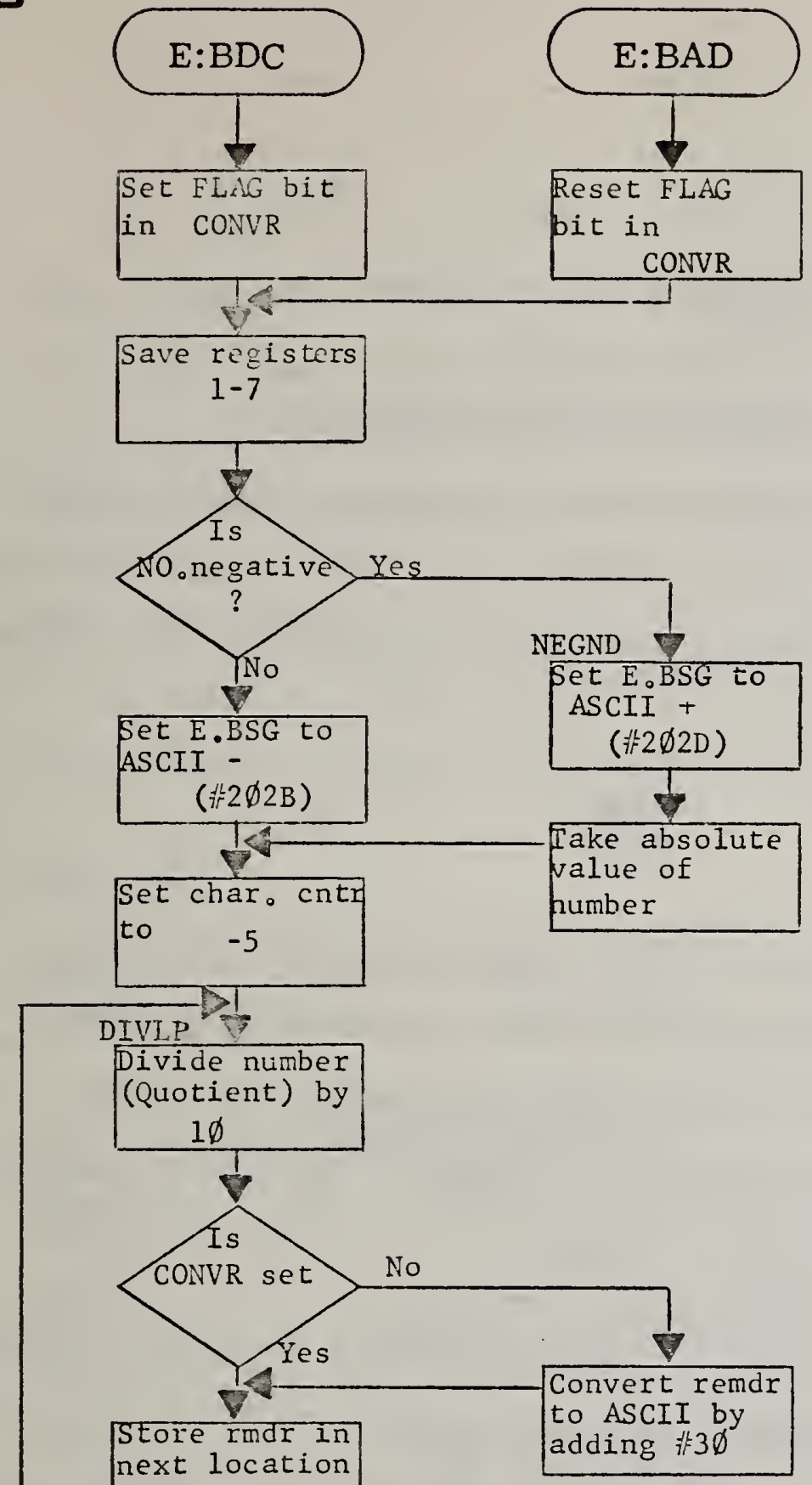

next location to ASCII by of $E_{0} B D F$

Increment Char Counter

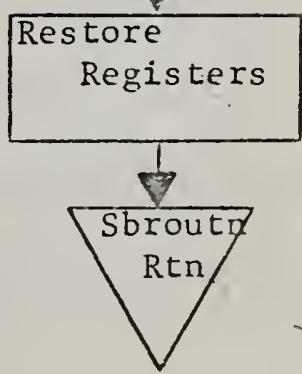


25. E\$FRT

25.1 Functional Description

25.1.1 E:FRT (Reformat Real Time for Output Subroutine)

NUSTAD generally includes the time of message generation in most of the messages entered into the station log. Those message generating programs normally call E:FRT to accomplish this function. The calling sequence is:

$$
\begin{array}{ll}
\text { BLM, 14 } & \text { E:FRT } \\
\text { DFC } & \text { STORE }
\end{array}
$$

$E: F R T$ reads the current $B C D$ time from the $W T C$, rearranges the characters, converts them to ASCII values, and stores them in five computer words starting at the location specified by the second instruction of the calling sequence. Control is returned to the calling program when the conversion is complete.

\subsubsection{E:FCT (Reformat BCD Time Value From Memory for Output Subroutine)}

Several NUSTAD programs store a time value in the BCD format for a period of time before that value needs to be output in a message. E:FCT is a variation on E:FRT which takes the $B C D$ formatted time from memory locations E.TMI and E.TM2 rather than reading the time from the WTC. The calling sequence is:

$\begin{array}{ll}\text { BLM, } 14 & \text { E:FCT } \\ \text { DFC } & \text { STORE }\end{array}$


E:FCT goes directly to E.TM1 \& T.TM2 to get the BCD time. It shares coding with E:FRT to reformat this value to ASCII characters and to store them in five computer words starting at the location specified by the second instruction of the calling sequence. Control is returned to the calling program when the conversion is complete.

\section{$25.2 \quad$ Data Formats}

The output of either E:FRT or E:FCT is stored in five memory locations with two ASCII characters per location, in the following form:

\section{DDD TTTTT}

WHERE: $\quad$ indicates a digit of DOY

I indicates a digit of TOD

25.3 Output Parameter Descriptions

PARAMETER SIZE DESCRIPTION

E:FRT Entry point for subroutine to convert real time to ASCII characters for output.

$E: F C T$

Entry point for subroutine to convert $B C D$ formatted time from memory to ASCII characters for output.

E.TM7

E.TM2

2

$B C D$ formatted time value converted by $E: F C T$.

25.4 Internal Data Descriptions

PARAMETER SIZE DESCRIPTION

SAVER

6 Register storage area. 


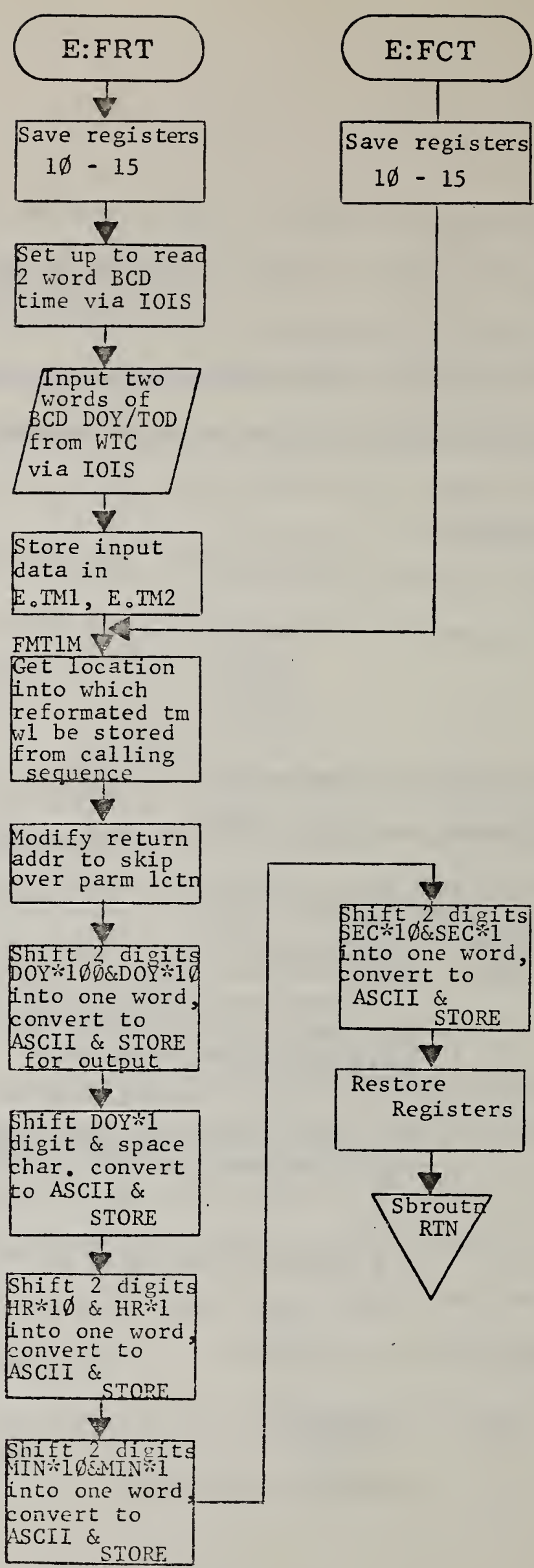




\subsection{Functional Description}

NUSTAD stores most values for time in the BCD format. This is the format under which time is read from the WTC, and conversion from this format to ASCII characters for output is very straightforward. However, the processor cannot perform computations with the time value in a packed $B C D$ format, so the following executive support subroutines are included in NUSTAD to permit conversion between the BCD and binary time formats and to perform the most common computation using the time values.

\section{1 .1}

The waveform analysis programs have frequent need to know how much time has elapsed since the signal onset. When onset was discovered, both the BCD time (in U.OTB) and the binary time (in U.OTM) were saved. Any program which requires the time difference between onset time and a $B C D$ formatted time value calls E:DIF where the time difference in seconds is computed and returned to the calling program. E:DIF also indicates the absence of a signal in progress by adjusting the return address. The calling sequence is:

$\begin{array}{lll}\text { BLM } 74 & \text { E:DIF } & \text { Sensor channel designated in R6 } \\ \text { DFC } & \text { ADDRS } & \text { Address of BCD value } \\ \text { BRU } & \text { NO SIG } & \text { Return if no signal in progress } \\ \ldots & \ldots \ldots . & \text { Return with time difference in R12, RT3 }\end{array}$


Upon entry, E:DIF checks the onset time (U.OTM) for the sensor channel specified in R6. If it is zero, there is no signal in progress, and E:DIF returns control to the calling program. If the onset time is nonzero, $E$ :DIF recovers the $B C D$ formatted value from the location specified by the calling sequence and converts that value to its binary equivalent. The difference between the onset time and converted value is computed and adjusted if the time span overlaps the $24 \varnothing 0$ hour. The return address is adjusted and control is returned to the calling program with the time difference (in seconds) in RT2, R13.

If a program requires the time difference between a binary value and a BCD time when the binary value is not a signal onset time, E:DiF can be used to store the binary time value in the two memory locations imediatedly following the U.OTM storage area. This two-word time buffer is called U.DTM. Setting the channel designation in R6 to ${ }^{14} 1 \emptyset$ will cause E:DIF to compute the time difference as if a fifteenth sensor channel existed and the time elapsed since onset on that channel were required.

\subsubsection{E:COT (BCD Formatted Time to Binary Equivalent Conversion Subroutine}

When an applications program needs to convert a $B C D$ time value to its binary equivalent, as to supply U.DTM with a binary value prior to calling E:DIF, E:COT can be called to perform the conversion. The calling sequence is: 
BLM, 14

DFC
E:COT

ADDRS
Address of $B C D$ value to be converted

E:COT retrieves the $B C D$ value from the location specified, converts the value to its binary equivalent and returns control to the calling program with the result in R12, R13.

\subsection{Computational Methods}

The BCD time value is formatted as illustrated in Figure 16.4. The conversion from this format to the binary equivalent, the format of which is illustrated in Figure 16.5, is made by the following computation:

$$
\mathrm{T}_{\text {bin }}=\left(\left(\left(\left(\left(D_{\emptyset} \times 7 \emptyset+D_{1}\right) \times 6+D_{2}\right) \times 1 \emptyset+D_{3}\right) \times 6+D_{4}\right) \times 1 \emptyset+D_{5}\right)
$$

where: $D_{\emptyset}$ is the hours $\times 1 \emptyset$ digit

$D_{1}$ is the hours $\times 1$ digit

$D_{2}$ is the minutes $\times 10$ digit

$\mathrm{D}_{3}$ is the minutes $\times 1$ digit

$D_{4}$ is the seconds $\times 10$ digit

$D_{5}$ is the seconds $\times 7$ digit

26.3 Input Parameter Descriptions

PARAMETER PROGRAM DESCRIPTION

U.OTM U\$DAT

Signal onset time in binary format. 
26.4 Output Parameter Descriptions

PARAMETER SIZE DESCRIPTION

$E: D I F$ Entry point for time difference computation subroutine.

$E: C O T$ Entry point for time format conversion subroutine.

26.5 Internal Data Descriptions

PARAMETER SIZE DESCRIPTION

SAVER 7 Register storage area.

FLAG $\quad 1 \quad=1$ if entry made through $E: C O T ;=\emptyset$ if through E:DIF 


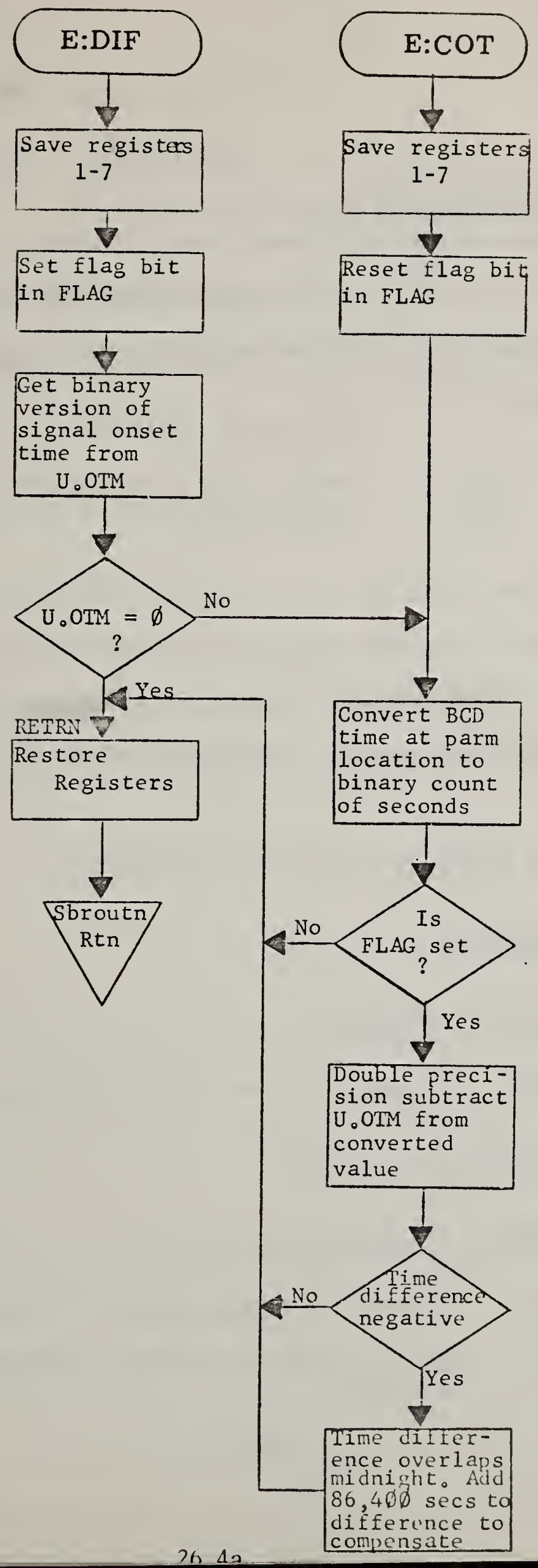




\section{E\$FMF}

\section{$27.1 \quad$ Functional Description}

When NUSTAD needs to output a phase value, the executive support subroutine E:FMF is called to reformat that value from its internal storage format (Figure 16.3) to a decimal form indicating microseconds. The calling sequence is:
$B L M, 15$
$E: F M F$
Phase value to be converted in $\mathrm{R} 12$
DFC
STORE
Address where result to be store

E:FMF assumes the value in $R 12$ is a phase formatted value, converts that binary number to a decimal value, and stores the corresponding ASCII characters in five computer words starting at the location specified in the calling sequence. The format of the resulting output is: $\pm \varnothing \emptyset \emptyset \emptyset . \emptyset \emptyset$

which represents a signed phase value in microseconds.

\section{$27.2 \quad$ Called Subroutines}

SUBROUTINE PROGRAM FUNCTION

E:BAD E\$BDC Converts binary value to ASCII decimal for output

\subsection{Input Parameter Descriptions}

PARAMETER PROGRAM DESCRIPTION

$\begin{array}{lll}\text { E.BSG } & E \$ B D C & \text { Sign of the output to call to } E: B A D \\ \text { E.BDG } & \text { E\$BDC } & \begin{array}{l}\text { Buffer for ASCII characters generated by call } \\ \text { to } E: B A D\end{array}\end{array}$




\subsection{Output Parameter Descriptions}

PARAMETER SIZE DESCRIPTION
$E: F M F$
Entry point to phase reformatting subroutine.

27.5 Internal Data Descriptions

PARAMETER SIZE DESCRIPTION

SAVER $\quad 7$ Register storage area. 


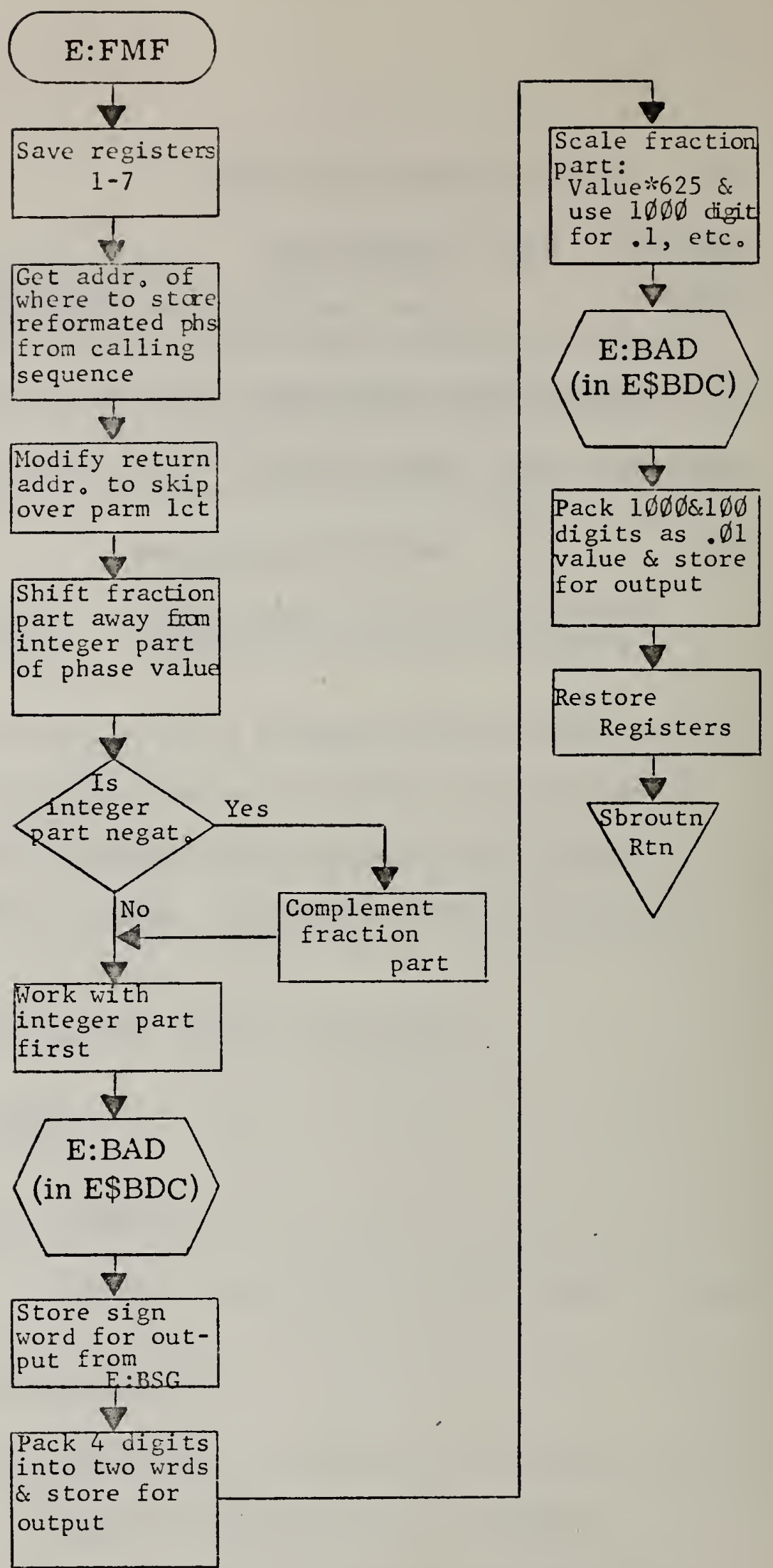


28.

E\$SRT

28. 1 Functional Description

E:SRT is an executive support subroutine to compute the positive square root of a double precision binary value. The double precision value is entered in $\mathrm{R} 12, \mathrm{R} 13$ and the subroutine is called by the following sequence:
BLM, 14
$E: S R T$
D.P. value in $R 12, R 13$

The positive (single precision) square root is returned in RT3.

\subsection{Computational Methods}

The square root is computed using Newton's method of tangents. The process is iterative and uses the following scheme:

$$
x_{n+1}+\frac{\left(A / x_{n}\right)+x_{n}}{2}
$$

where: $x_{n}$ is the current estimate of the square root

$x_{n+1}$ is the next estimate of the square root

$A$ is the argument of of the function

To aid in convergence of the successive estimates, the initial value $x_{\emptyset}$ is chosen to be close to the final value. That initial estimate is made thus ly: 


$$
x_{\emptyset}=\left(A / 2^{(n / 2)}\right)
$$

Where: $n$ is the number of significant bits required to represent $A$ in binary.

(Note: $n / 2$ is truncated to give an integer)

28.3 Output Parameter Descriptions

PARAMETER SIZE DESCRIPTION

$\begin{array}{lll}\text { SAVER } & 7 & \text { Register storage area. }\end{array}$ 


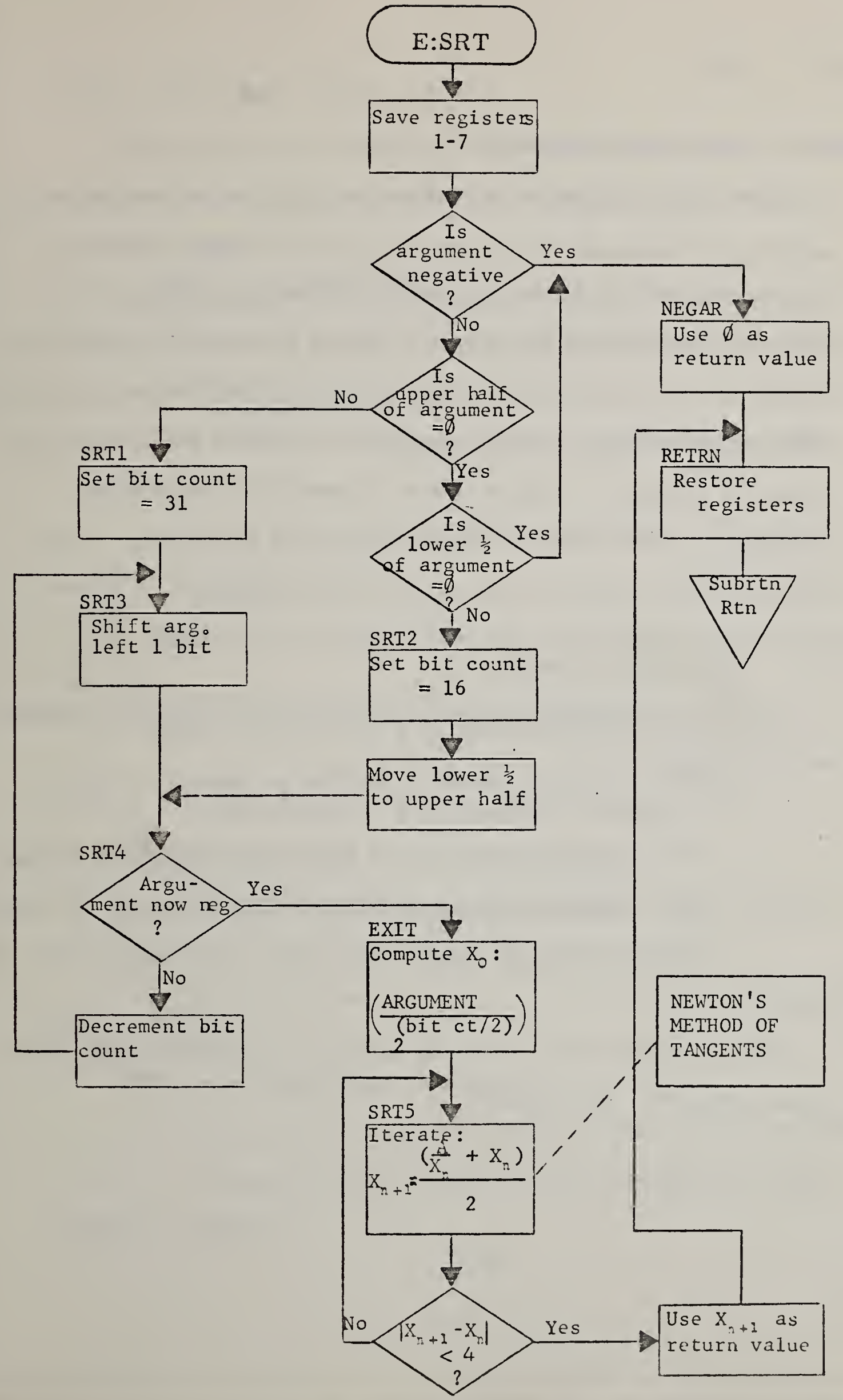




\subsection{Functional Description}

F\$FMT checks the DRFUD tape data for invalid bit configuration by checking for known zero bit locations in the DRFUD data stream. If a bit is found to be set at one of these locations, the tape track containing the improper bit state is Tabeled and output to the ASR-35 teletype for the operator's information. By working in conjunction with F\$DMP, the entire data scan containing the bit error is displayed on the ASR-35 teletype to further aid the operator in locating the discrepancy. This routine is used to check the reliability of the DRFUD data and is therefore run in the background mode.

\subsubsection{F:FMT (DRFUD format)}

$F: F M T$ is the operator entry point to F\$FMT from E:BSK and performs two basic tasks:

a. Interprets the operator's intentions by

(1) Checking status of bit $\emptyset$ of switch register. (Figure 29.1)

(2) Requesting number of blocks of DRFUD data to be checked.

b. Sets a counter to control the number of blocks of data checked.

F:FMT is entered only after the operator requests a format check action using DFC: as a keyword. 


\subsubsection{F:CHK (DRFUD Checking Routine)}

F:CHK performs the checking function and enters an error locating routine if an error is detected. The sequence is executed using the following steps.

a. Compares the DRFUD data, one word at a time, to a known fixed format. If any ones compare, an error is present and a branch is made to an error locating routine.

b. Goes through a word mask and a bit shifting sequence to locate the bit in error.

c. Outputs a message telling the operator which tape track contains the error.

d. Executes a branch to $F: D D D$ and displays the scan containing the error.

e. Checks bit position $\emptyset$ of switch register to determine if operator intends to continue checking same block of data.

\subsubsection{F:TRD (Tape Read)}

F:TRD sets required parameters and initiates a mag tape read via B:IOR. Data is stored in MTBUF (Mag Tape Buffer).

\subsubsection{F:USE}

F:USE serves two primary functions:

a. Responds to a need for a mag tape read by calling F:TRD.

b. Transfers 55 byte blocks of data from MTBUF to FUDBF (DRFUD Data Buffer). 


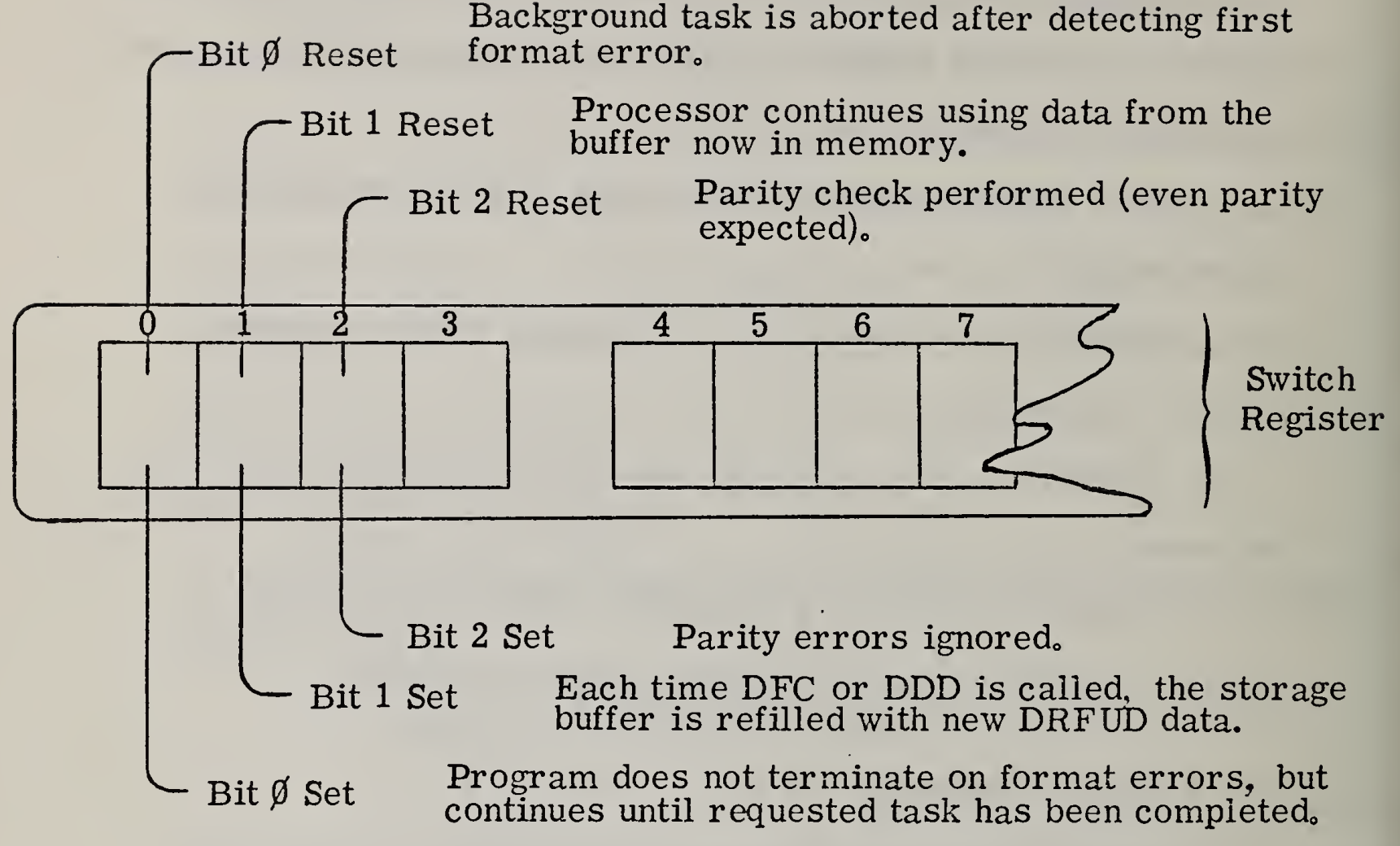

Figure 29.1

Switch Register Options 
$29.1 .5 \quad$ F:CVT

$\mathrm{F}: \mathrm{CVT}$ is a general purpose routine that performs text analysis on and converts operator responses to ASCII.

$29.2 \quad$ Called Subroutines

SUBROUTINE PROGRAM FUNCTION

E:SKD E\$SKD Scheduler reentry point.

$B: I O R \quad B \$ T S I \quad B a c k g r o u n d$ program I.0. request routine.

F:DDD F\$DMP DRFUD data display routine.

29.3 Computational Methods

\subsubsection{Error Detection Scheme}

Error detection is accomplished by checking the DRFUD data for bits set in known zero locations. Once an error has been detected, the word is examined by extracting the data and checking for the set bit position through a series of shifts. Once the set bit position has been located, the tape track containing it is labeled and output to the ASR-35 teletype.

29.4 Data Formats

29.4.1 DRFUD Tape Format

Input requests by the operator in response to "NO. OF BLOCKS TO BE CHECKED" should be a positive integer less than 32,767 and are entered as explained in Appendix III. 
DRFUD data is read from magnetic tape in the format shown in Figure 29.3. It is repacked, two bytes to a word, and is stored in MTBUF. The same format is kept when the data is transferred to FUDBF. Zeros are inserted in the last byte position of FUDBF to insure that any error detected is not the result of this byte containing previously set values.

29.5 Input Parameter Descriptions

PARAMETER PROGRAM DESCRIPTION

B.STT E\$SKD Background status flag, set to $\emptyset$ for no task in progress.

E.35I E\$T35 Address of first character of input from ASR-35.

29.6 Output Parameter Descriptions

PARAMETER SIZE DESCRIPTION

F:FMT

Entry point for DRFUD format check routine (see Section 29.1.1).

F:CHK Entry point to the format checking sequence (see Section 29.1.2).

F:TRD Entry point to the tape read initiator (see Section 29.1.3).

F:USE Entry point for operator response conversion routine (see Section 29.1.5).

F.DBF 1 External reference name for the DRFUD data buffer (FUDBF).

F.MTP 1 Location pointer for MTBUF. 
29.8 Internal Data Descriptions

PARAMETER SIZE DESCRIPTION

NUBK $16 \quad$ "NO. OF BLOCKS TO BE CHECKED" message.

BADNO 16 "INVALID CHAR, TASK ABORTED" message.

X.VAL $\quad 12$ "FORMAT ERROR BIT 8" message.

FINSH $\quad 1 \emptyset \quad$ "TASK COMPLETED" message.

QUIT 12 "FORMAT CHECK TERMINATED" message.

TAB 6 Table of mag tape track labels used to indicate errors present.

MTBUF $\quad 794 \emptyset \quad$ Mag tape buffer.

FUDBF 28 Working buffer for DRFUD data.

FRS $\quad 7 \quad$ Temporary storage for contents of registers 1 through 7. 


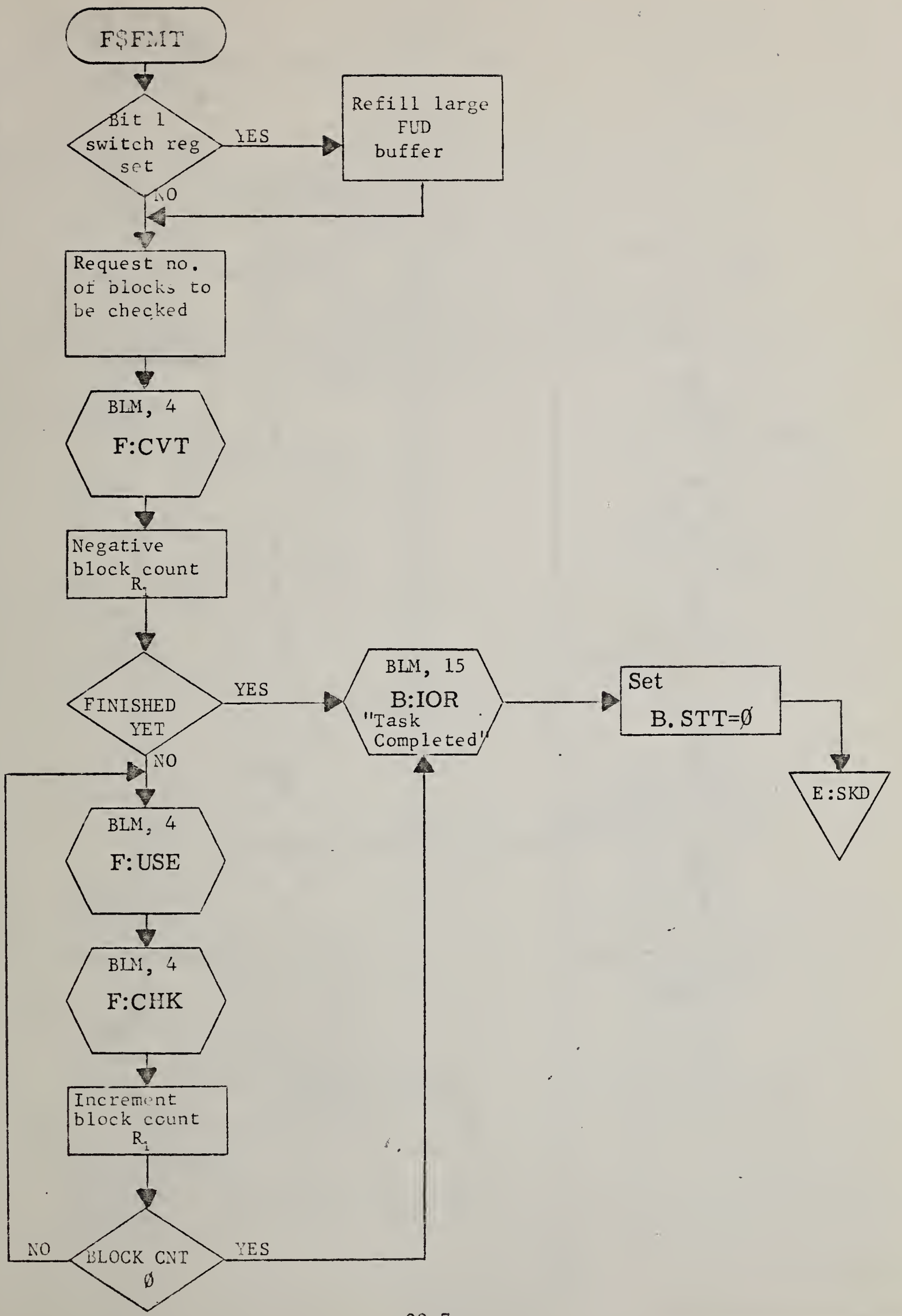




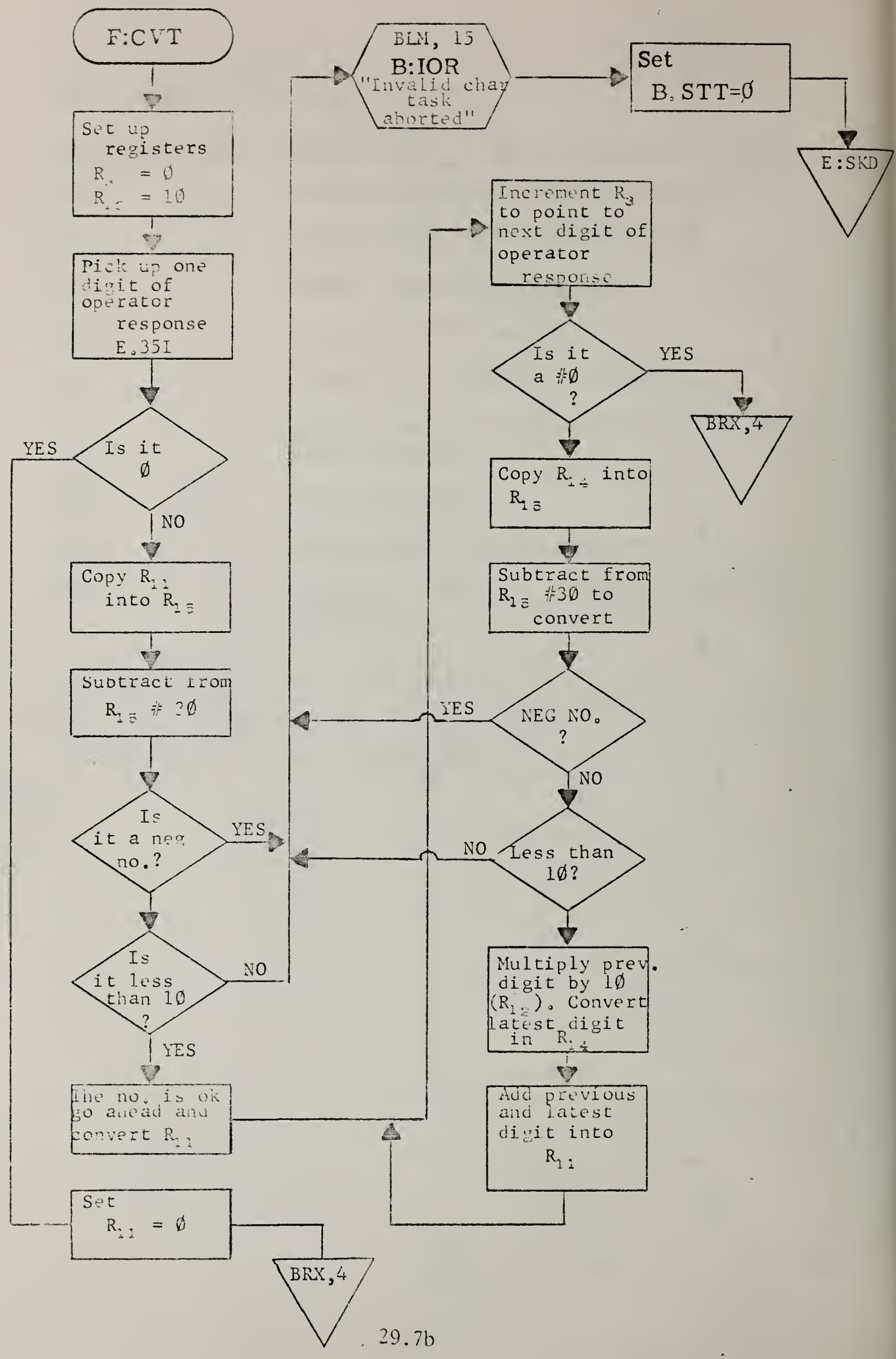




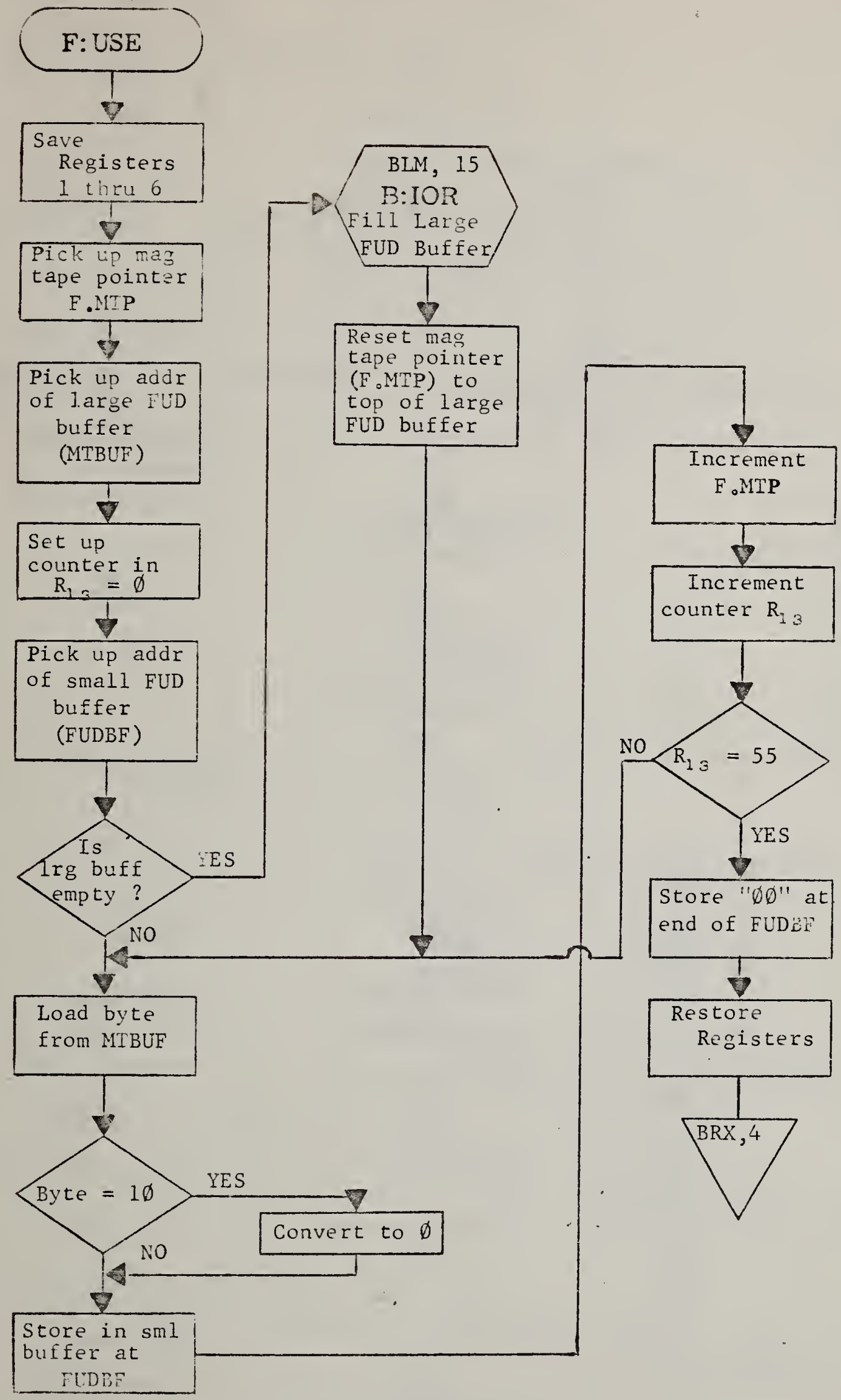

$29.7 \mathrm{c}$ 


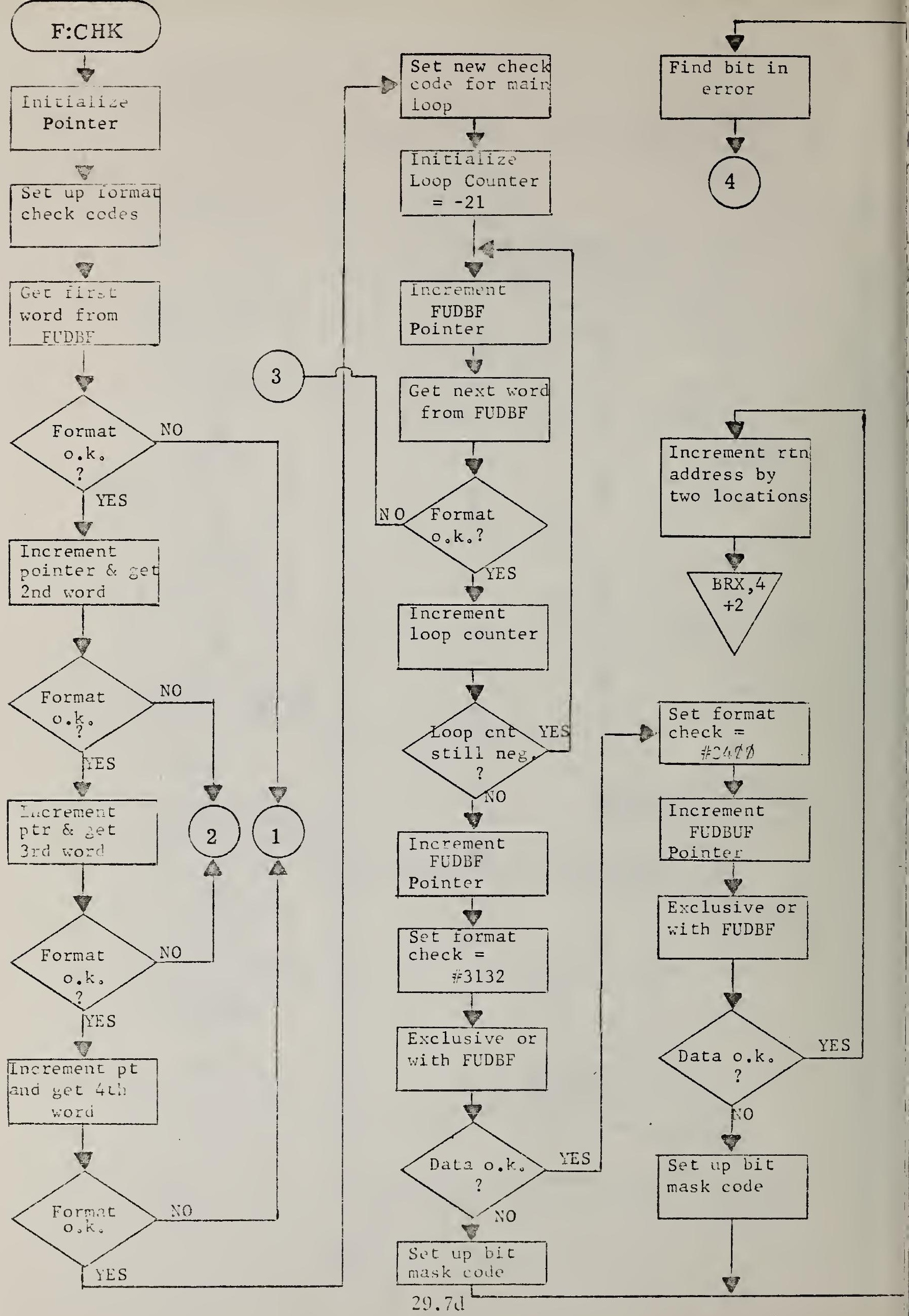


$3 \emptyset$.

\section{$3 \emptyset .1 \quad$ Functional Description}

F\$DMP enables the operator to display DRFUD data on the ASR 35 in a usable format. He can specify the parameters of the display as either time or number of data scans to be displayed. The data to be displayed is taken directly from FUDBF using byte addressing it is reformatted in the progress and is displayed in two lines, the first containing time and location information and the second being made up of the phase and amplitude values of the eight recorded channels. Before being displayed, the data is examined by $\mathrm{F}: \mathrm{CHK}$ and errors are handled as explained in Chapter 29.

The program is entered in F:DMP from E:BSK bit $\emptyset$ of the switch register is checked to determine whether or not a new block of data is to be read from mag tape. The initial dialog is then started to determine which option the operator wishes to use. The options are listed in the table below.

1. START TIME AND STOP TIME.

2. START TIME AND NUMBER OF DATA BLOCKS.

3. START IMMEDIATE AND NUMBER OF DATA BLOCKS.

Table $30.1 \quad$ Operator Display Options 
The conversation is carried on via the B:IOR subroutine of E\$TSI. Information from the operator is retrieved from E.35I (E\$T35) at various points in F:DMP. F:FST first determines if the operator wants a start time, if not, it outputs a message requesting "NUMBER OF BLOCKS TO BE DUMPED." Operator response to this inquiry is converted to ASCII and set in a counter (BLKBF) that limits the display length. Each time a data scan is displayed, F:USE is entered and new data is transferred from MTBUF to FUDBF. If a start time is desired (option 1 or 2), a message is output stating "SPECIFY START TIME DESIRED." The operator's response is converted to binary (PACK) and held in NGSTR and NGSTR+1. The contents of MTBUF are scanned via FUDBF until the difference between the time requested and the time of the data scan is zero. The contents of MTBUF are automatically updated as explained in Chapter 29. A format check is performed prior to each time comparison to insure that the data contained in the time locations of FUDBF are not simply phase and amplitude data misplaced because of a dropped character. In the event that the DRFUD tape data was recorded sometime after the start time requested, a message is output stating this fact and control is returned to NUSTAD. Once the start time has been located, a message is output requesting a stop time (option 1) or a number of data blocks (option 2). The stop time is converted to a number of data scans ( $1 /$ second) and stored in BLKBF. The number of blocks is converted from ASCII and stored in BLKBF. The display is continued until BLKBF reaches zero. 


\section{$30.1 .1 \quad$ F:DMP}

$F: D M P$ is the program organizer for F\$DMP. It serves three basic purposes:

a. Checks status of bit position $\emptyset$ of switch register to determine if the operator wants to refill MTBUF with new data.

b. Calls for a start time message which gives the operator the three options previous Ty mentioned (Section 30.1).

c. Keeps track of the amount of data that has been displayed and returns to E\$SKD when the assigned task is completed.

\section{$3 \emptyset .1 .2 \quad \underline{F: D D D}$}

F:DDD simply repacks the DRFUD data one byte at a time into the format shown in Figure 30.1. The data is stored in two separate Tocations, HEADR and DATA. HEADR contains time and location -information and DATA contains phase and amplitude for eight DRFUD channels plus three fixed control characters that appear at the end of a data block. The two information blocks are output one at a time to avoid tying up the output channe1 with large message outputs.

\section{$30.1 .3 \quad$ F:FST}

F:FST is the routine that sets the DRFUD tape to the desired starting position. It also performs some text analys is on operator responses to machine requests. 
$30.1 .4 \quad$ F:TIM

F:TIM converts both operator requested DOY and DRFUD DOY to binary for comparison, then performs a greater than or equal to check to see if the tape is positioned at the correct DOY. If the DOY requested is less than the DOY contained on the DRFUD tape, a message (PASTD) is output informing the operator of this fact. If the DOY's are the same, a branch is executed to F:BBT which converts the DRFUD time data to binary. A comparison is made between the DRFUD time and the requested time resulting in either an additional read to advance the mag tape or an output message (PASTT) informing the operator that the tape is positioned past the time desired. Once both comparisons are satisfied, a request is output for stop time information. The stop time requested is simply converted to a number of data scans ( $1 /$ second) and stored in BLKBF. If during the time search, an error is detected, the task is aborted and control is returned to $E: S K D$.

$3 \emptyset .1 .5 \quad \underline{F: B B T}$

$\mathrm{F}: \mathrm{BBT}$ converts $\mathrm{BCD}$ to binary time and returns with the result in registers 12 and 13 . 
$\mathrm{F}:$ CHK

F\$FMT

Performs the checking sequence in F\$FMT and enters an error locating routine ( $F . B A D)$ if an error is detected.

B:IOR

E\$TSI

Background program I.0. request routine.

F:CVT

F\$FMT

$E: S K D$

$E \$ S K D$

F:USE

F\$FMT
Routine to convert operator response to ASCII.

Scheduler reentry point.

Routine that transfers data from MTBUF to FUDBF.

30.3 Data Formats

30.3.1 DRFUD Data Formats

DRFUD data is input in the format shown in Figure 29.1 and is output in the format shown in Figure 30.1 . If an error is detected, the standard output format will be preceded by an error statement as shown in Figure 30.2 .

30.3.2 Operator Response (Time Data)

Operator response to a time request is changed to a format compatible with DRFUD data.

$$
\begin{array}{ll}
\text { (DOY)(Time) } & \text { (Time) } \\
123123456 \text { is repacked to } 123456 \text { (DROP DOY) }
\end{array}
$$

30.3.3 Operator Response (Parameter Format)

Operator responses to machine request for parameter information are outlined in Appendix III. 


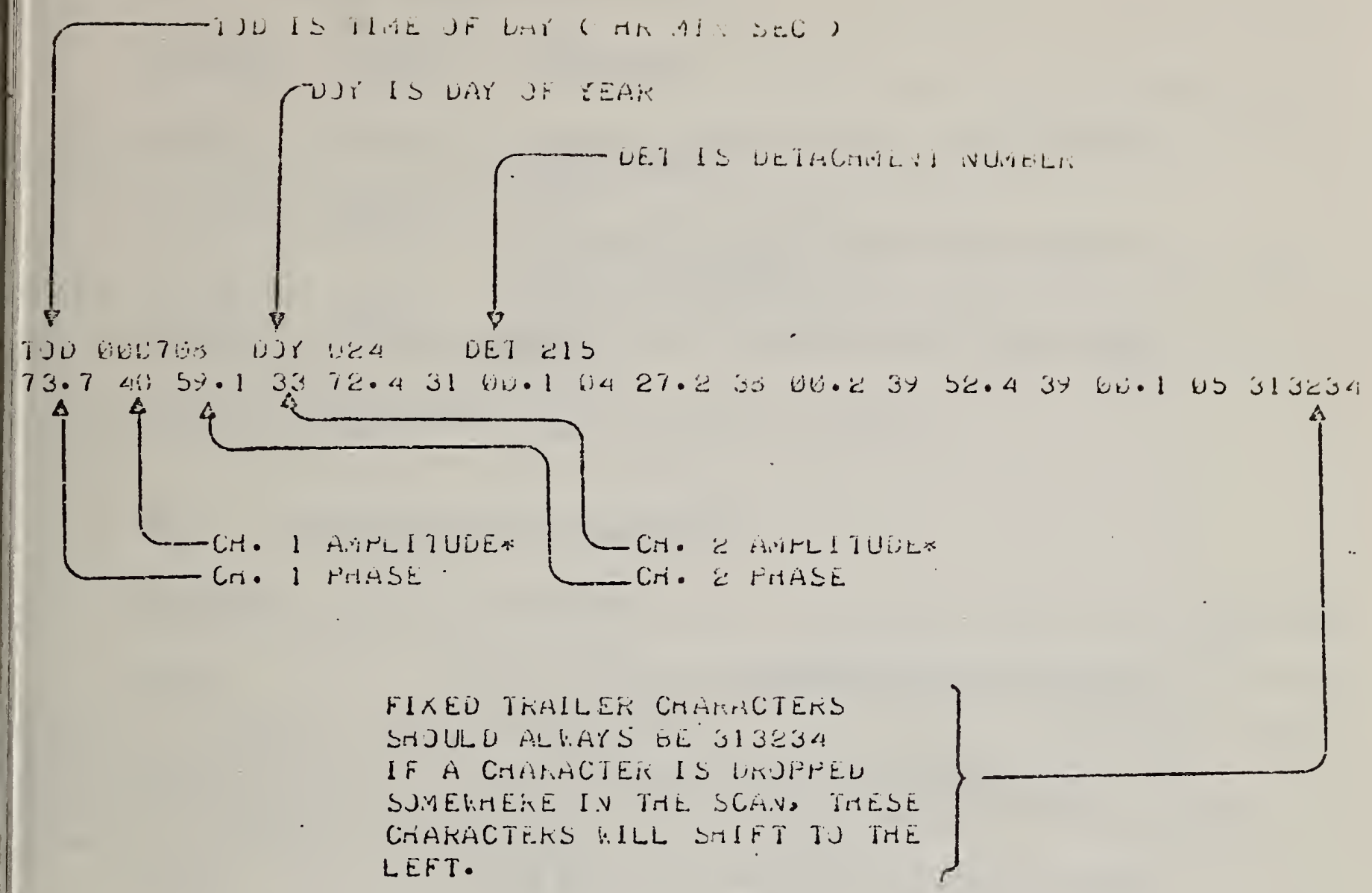

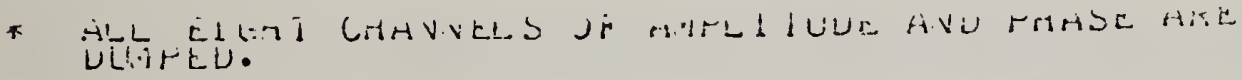

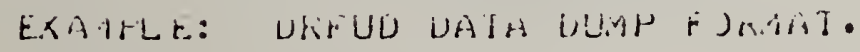

$$
\text { F咲. } 36.1
$$


$\times$ UrC:

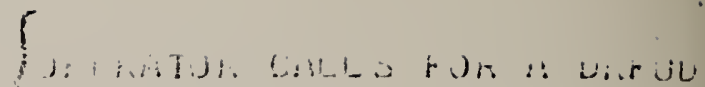

f

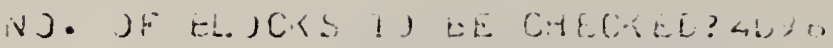

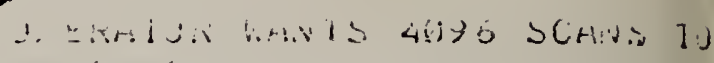

tos Coratiou.

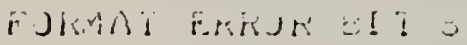

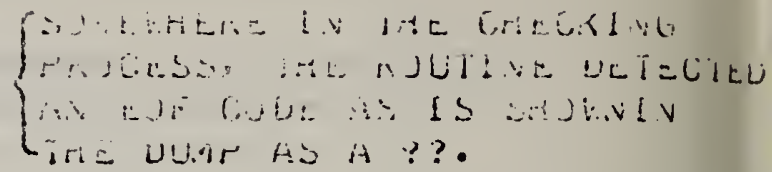

TJU ??l一ES 13): 124 UET E15

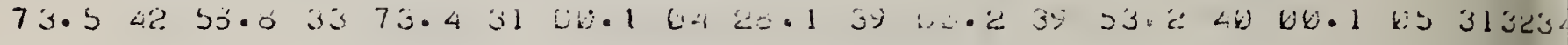

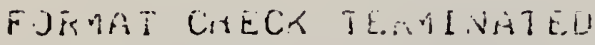

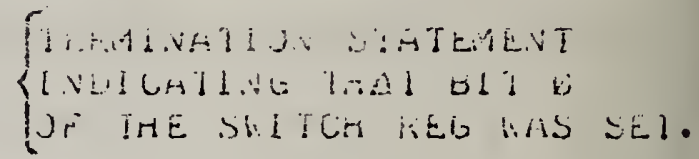

$\times \quad O F C:$

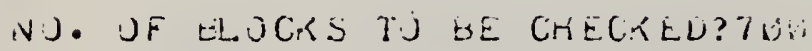

TASK COMLETES

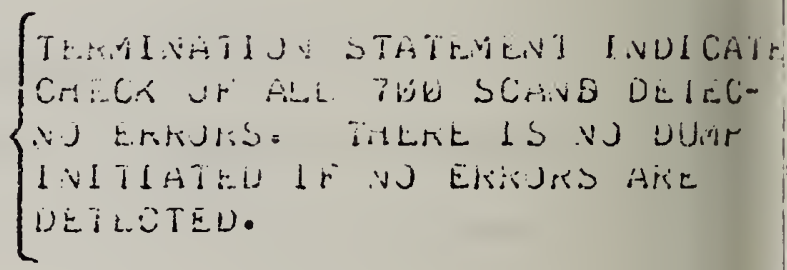

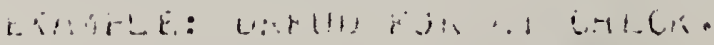

Fig. $3 \% .2$ 
30.4 Input Parameter Descriptions

PARAMETER PROGRAM DESCRIPTION

$\begin{array}{ll}\text { F.DBF F\$FMT } & \begin{array}{l}\text { External reference name for the DRFUD data } \\ \text { buffer (FUDBF). }\end{array}\end{array}$

B.STT E\$SKD Background status flag, set to $\emptyset$ for no task in progress.

E.34I E $\$$ Ad35 Adress of first character of input from ASR 35.

F.MTP F\$FMT Location pointer for MTBUF.

30.5 Output Parameter Descriptions

PARAMETER SIZE DESCRIPTION

F:DMP Entry point for DRFUD data display routine (Section 30.1 .1 )

F:FST Entry point for section of program that requests one of the three options (Section 30.7.3)

F:BBT Entry point for conversion routine to change $B C D$ to binary time (Section $3 \emptyset .1 .5$ ).

F:TIM Entry point to routine that finds the time requested on mag tape (Section $3 \emptyset .1 .4$ )

F:DDD Entry point to the routine that formats the data and displays it on the ASR 35 (Section 30.1.2)

30.6 Interna1 Data Descriptions

PARAMETER SIZE DESCRIPTION

BLKBF 1 Storage for a block count value.

SVDDD $\quad 13$ Temporary storage for registers 1 through 7 and 10 through 15. 
$30.6 \quad$ Internal Data Descriptions (continued)

PARAMETER SIZE DESCRIPTION

HEADR

18 "TOD 123456 DOY 123 DET 515" output message.

DATA

37 Phase and amplitude display message for eight channels of DRFUD data.

OPTS

20 "SPECIFY ACTION DESIRED, 1, 2 or 3." output message.

NOBLK

16

"NO. OF BLOCKS TO BE DUMPED" output message.

TIME

16

"SPECIFY START TIME DESIRED" output message.

QUIT

"SPECIFY STOP TIME DESIRED" output message.

FORM

T.MBF

6 Address of first $B C D$ word of time used in $F: B B T$.

RFCRS

7 Temporary storage for registers 1 through 7 .

OPDOY

1

NGSTR

2 Storage for operator requested time of day.

SAVER

7 Temporary storage for registers 1 through 7 .

PASTT

22

PASTD

22

NOGO1

19

BBTSV

7 Temporary storage for registers 1 through 7 . 


\section{F\$DMP}
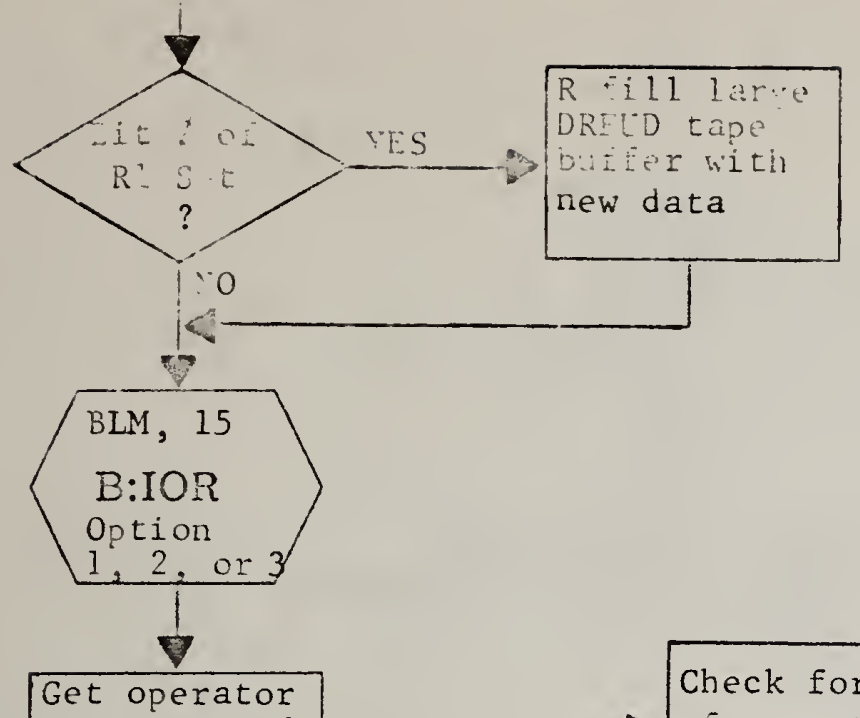

response and see what iie wants to do
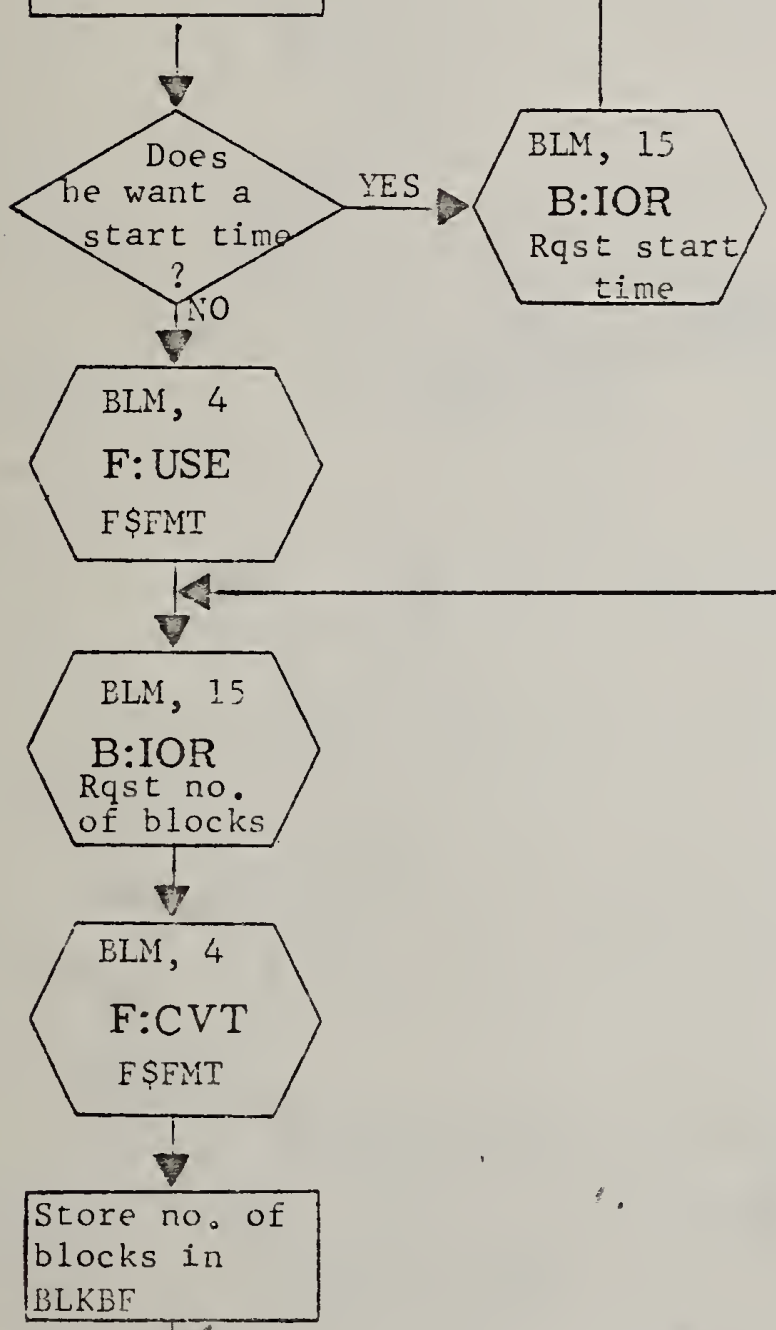

time
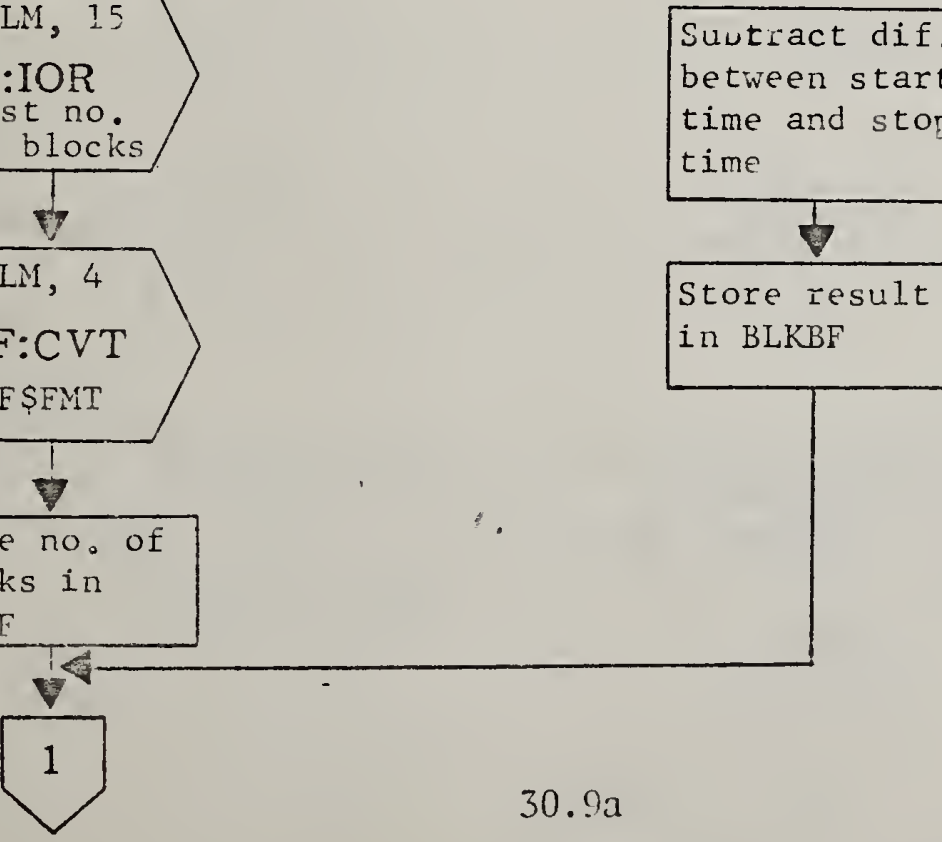

Check format of operato: response

\section{$\nabla$}

Repack oper. response and drop DOY in BLKBF 


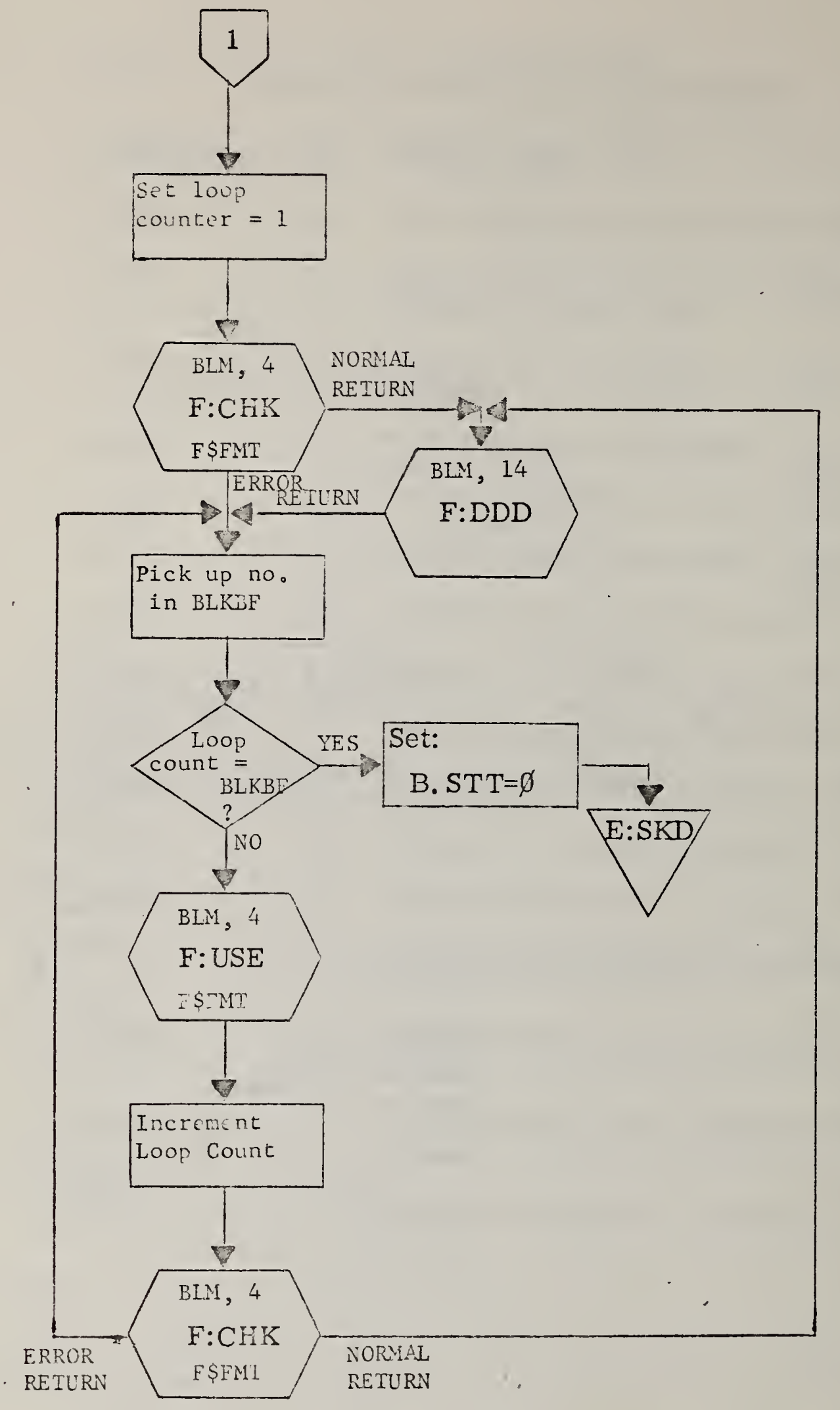




\section{F:TIM}

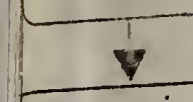

Gave rexister

thru 7
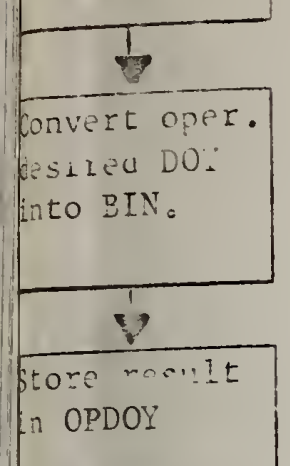

7

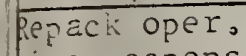
timf esponse and get it ready to convert.

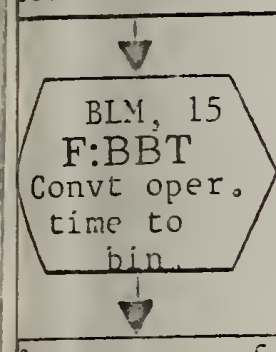

store neg. of result in :GSTR and NGSTR + 1

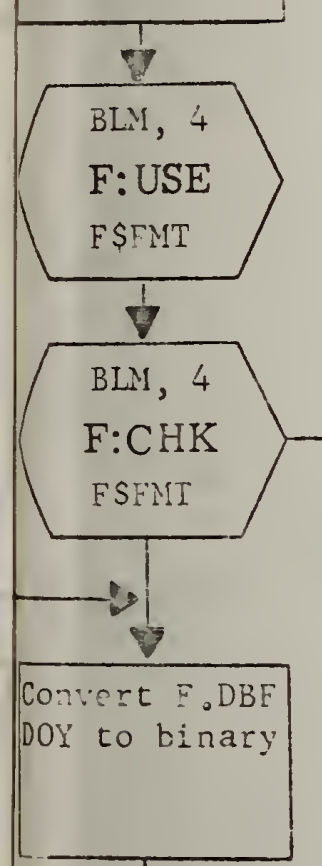

ERROR

RETURN

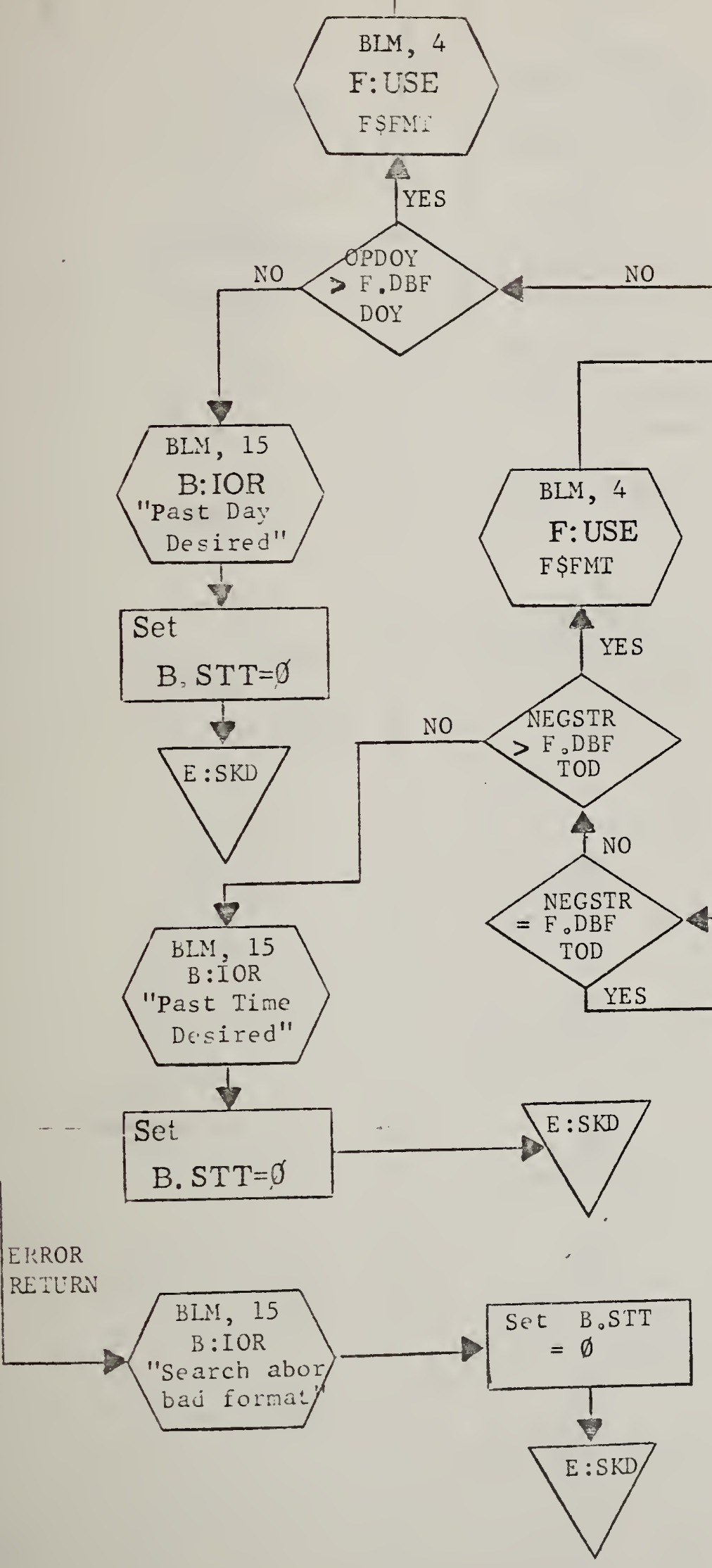

Comar

Do with OPDO

OPDOY

$t$

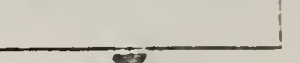

$=$

DOY

YES
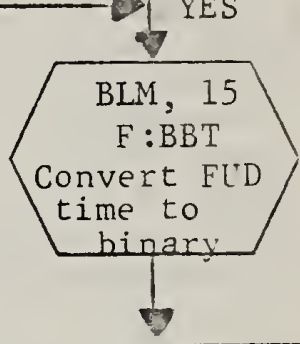

Pick up

NEGSTR \&

NEGSTR + 1

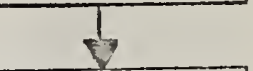

Compare F.DBF

TOD with

NGSTR \&

NECSTR + 1

(Dh1 Precis -) 


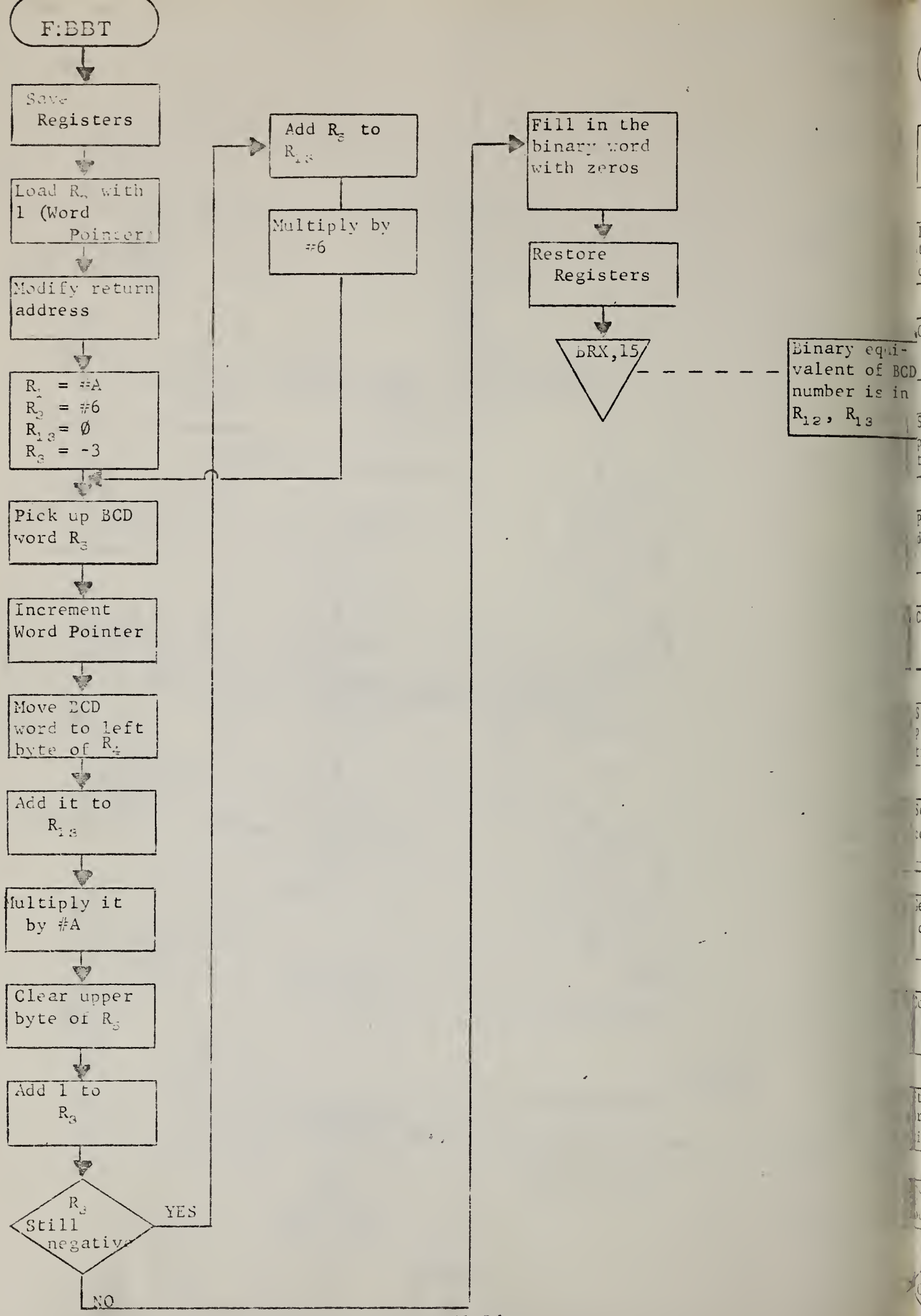




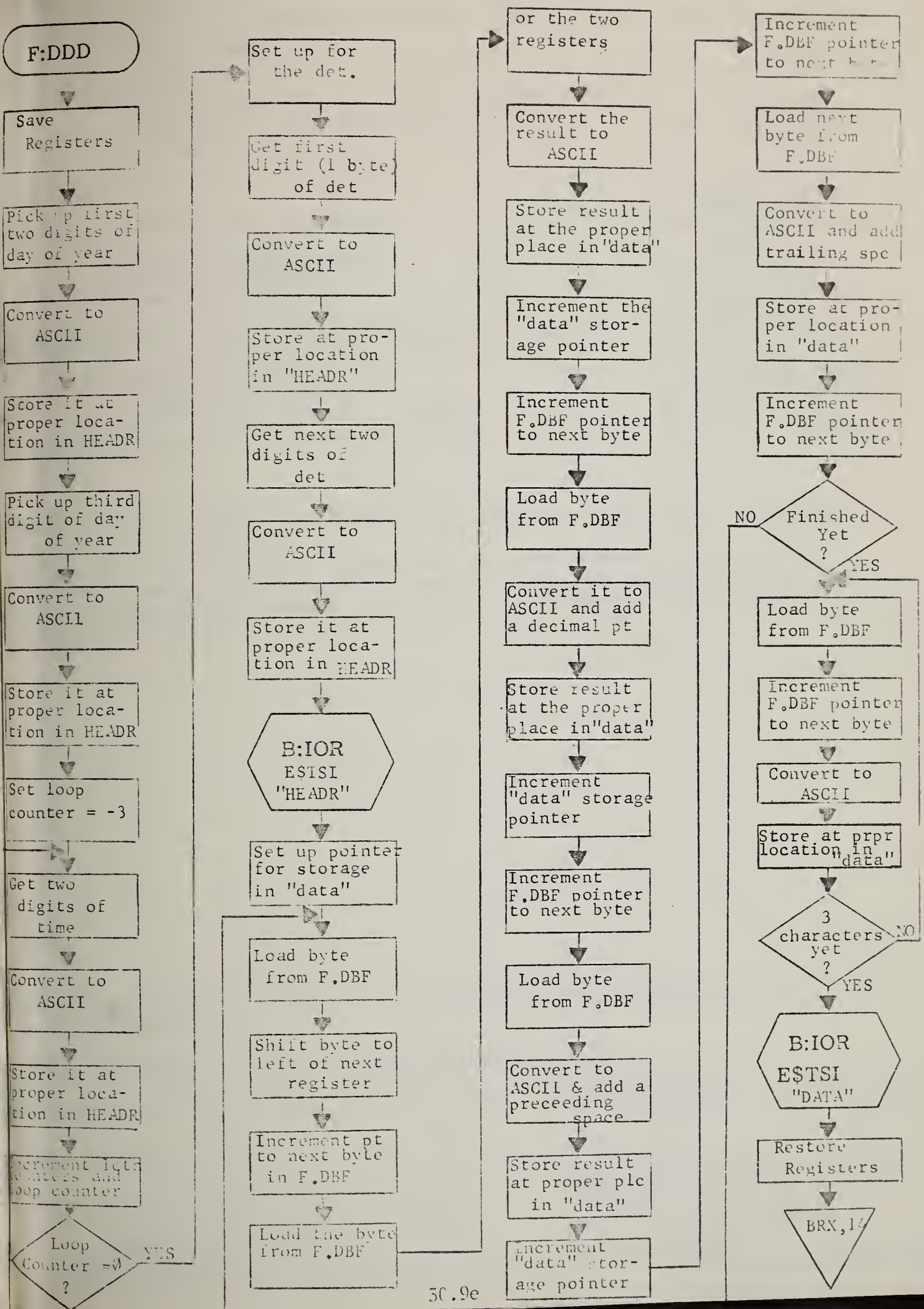





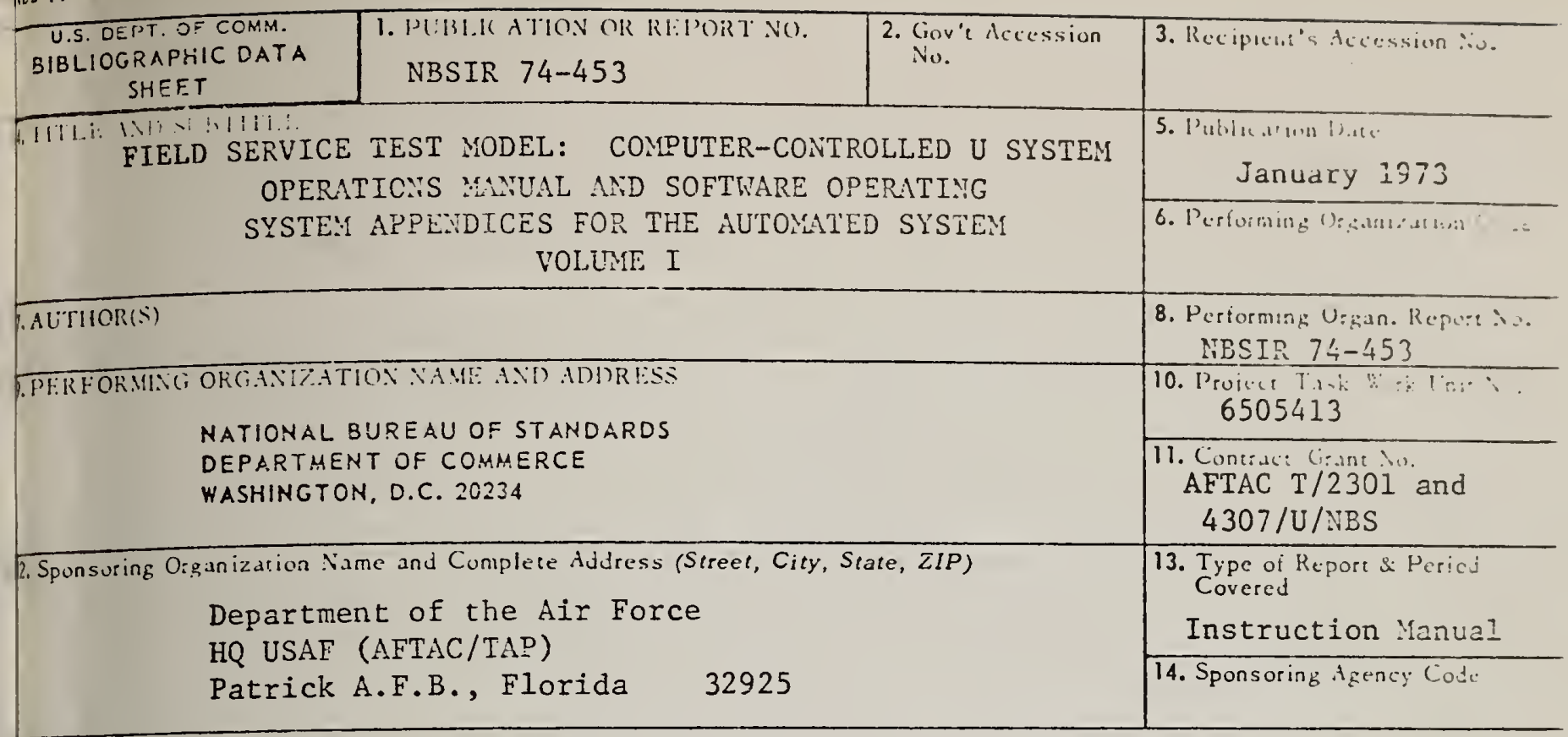

S. SLPP!.EAENTARY NOTES

6. AESTRACT (A 200-word or less factual summary of most significant information. If document includes a significant bibliography or literature survey, mention it here.)

Volume I of the "Automated U System" describes the software Iistings and the operator's manual. This volume is intended to instruct the station technician in. the procedure to load programs and operate, under computer control, the automated $U$ System.

1. KFY WORDS (six to twelve entries; alphabetical order; capitalize only the first letter of the first key word unless a proper name; separated by semicolons)

Operating system; program listings; software utilization.

1. AVAILABII.ITY

E Unlimited

TX For Officinl Disteiturion. No Not Release to NTIS

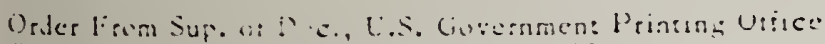

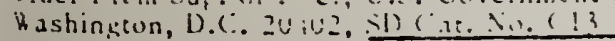

Order From Nacional Technical Iniurmation Service (NTIS) Springficld, Virginia 22151

\begin{tabular}{|c|c|}
\hline $\begin{array}{l}\text { 19. SECURITY CL.ASS } \\
\text { (THIS REPURT) } \\
\text { L'VCL ASTIEIED }\end{array}$ & $\begin{array}{c}\text { 21. NO. OF ! } A==5 \\
31 ?\end{array}$ \\
\hline $\begin{array}{l}\text { 20. B FCLKHI ( L.ASS } \\
\text { (THIS PACE) }\end{array}$ & $22 .+1:$ \\
\hline UNCLASSIFIED & \\
\hline
\end{tabular}






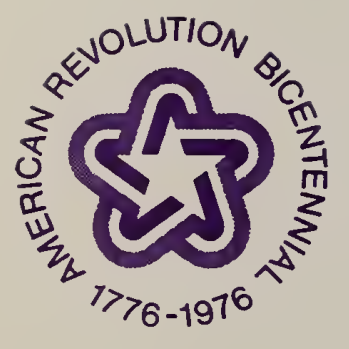

